\title{
Studies towards the Synthesis of a Heterobimetallic Zirconium Germanium Complex from Imidozirconocenes, \\ Germanimines and Germylenes
}

By

Peter Chapple

A thesis

Submitted to Victoria University of Wellington

In partial fulfilment of the requirements for the degree of

Masters of Science

In Chemistry

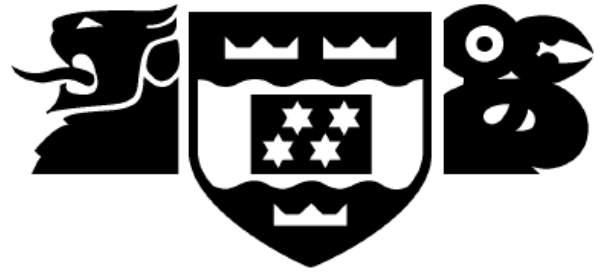

Victoria University of Wellington 


\section{Abstract}

Early-late transition metal heterobimetallic complexes with direct metal to metal interactions are desirable synthetic targets due to the complementary reactivity of the two different metals present in these compounds. The electron-rich late transition metal (often Rh, Ir, Fe, or Mo), and electron-poor early transition metal create an ideal environment for heterolytic bond cleavage in what is often termed 'cooperative reactivity'. This project aimed to synthesise a zirconium-germanium heterobimetallic complex based on a known heterobimetallic ligand scaffold; 1.

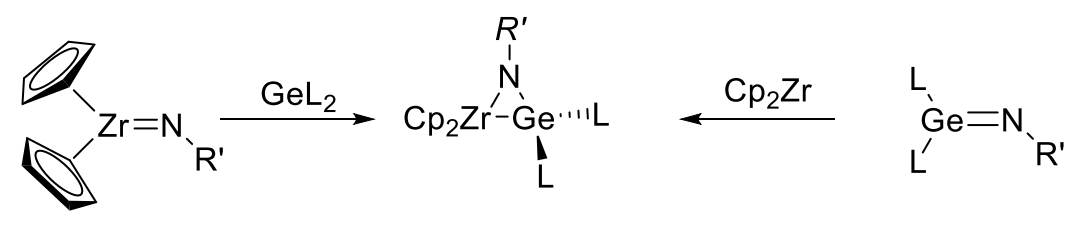

1

The synthesis of the desired heterobimetallic $\mathbf{1}$ was attempted using two different synthetic approaches. The first involved the investigating the reactivity between an unsaturated zirconium nitrogen bond (an imidozirconocene) and a germanium(II) source with a lone pair of electrons (known as a germylene). The second approach investigated the reactivity between an unsaturated germanium nitrogen bond (a germanimine) and a zirconium(II) source. In order to have the highest chance of success, a wide range of germanium and zirconium complexes were synthesised.

The novel germylenes include [Ge(NAPH $\left.\left.\left.{ }^{\mathrm{TMS}}\right)\right]\left(\mathrm{NAPH}^{\mathrm{TMS}}=\left[1,8-\left(\left(\mathrm{CH}_{3}\right)_{3} \mathrm{Si}\right) \mathrm{N}\right)_{2} \mathrm{C}_{10} \mathrm{H}_{6}\right]\right)$ and $\left[\mathrm{Ge}\left(\mathrm{BIAN}{ }^{\mathrm{Mes}}\right)\right.$ ] $\left.\left(\mathrm{BIAN}^{\mathrm{Mes}}=\left[\left(\left(2,4,6-\mathrm{Me}\left(\mathrm{C}_{6} \mathrm{H}_{2}\right) \mathrm{N}\right)_{2}\right) \mathrm{C}_{12} \mathrm{H}_{6}\right)\right]\right)$. These proved to be unreactive towards the imidozirconium species $\left[\mathrm{Cp}_{2} \mathrm{Zr}\left(\mathrm{NAr}{ }^{*}\right)(\mathrm{THF})\right]$ and $\left[\mathrm{Cp}_{2} \mathrm{Zr}(\mathrm{NDipp})(\mathrm{THF})\right]\left(\mathrm{Ar} *=\left(2,6-\left(\mathrm{C}_{6} \mathrm{H}_{5}\right)_{2} \mathrm{CH}\right)-4-\left({ }^{t} \mathrm{Bu}\right) \mathrm{C}_{6} \mathrm{H}_{2}\right)$, Dipp = $(2,6-$ $\left.\left.\left((\mathrm{CH} 3)_{2} \mathrm{CH}\right) \mathrm{C}_{6} \mathrm{H}_{3}\right)\right)$ as well as other amidozirconocenes. However, within these studies, the mixed coordination germanium species $\left[\left[\mathrm{Ge}\left(\mathrm{NAPH}^{\mathrm{TMS}}\right) \mathrm{Ge}(\mathrm{Bu})\left(\mathrm{NAPH}^{\mathrm{TMS}}\right)\right]^{[}\left[\mathrm{Li}\left(\mathrm{THF}_{4}\right)\right]^{+}\right] \quad$ and $\left[\left[\mathrm{Ge}\left(\mathrm{NAPH}^{\mathrm{TMS}}\right) \mathrm{Ge}(\mathrm{Me})\left(\mathrm{NAPH}^{\mathrm{TMS}}\right)\right]^{[}\left[\mathrm{Li}\left(\mathrm{THF}_{4}\right)\right]^{+}\right]$were synthesised. Density functional theory (DFT) molecular orbital calculations were used to help explain the observed reactivity.

With regards to the second approach, routes to new germanimine complexes such as [(HMDS) ${ }_{2}$ Ge(NMes)] $\left((\mathrm{HMDS})=\left(\left(\mathrm{CH}_{3}\right)_{3} \mathrm{Si}\right)_{2} \mathrm{~N}\right)$, Mes $\left.=\left(2,4,6-\mathrm{CH}_{3}\left(\mathrm{C}_{6} \mathrm{H}_{3}\right)\right)\right)$, were explored, and several methods for generating " $C p_{2} Z r$ " were examined. Although these conditions proved unsuccessful for generating 1 , the reaction between dialkyl zirconocene with azides to form novel zirconocene triazenido complexes was discovered and the sterics affecting the synthesis of new germanimine complexes was investigated. 


\section{Acknowledgements}

Primarily I would like to thank my research supervisor Dr. Robin Fulton, for her direction and support over the last two years. Without her guidance and patience, I would certainly not have been able to complete this thesis. Robin's flexibility and openness towards suggestions always made me feel like I had real influence and ownership of my research and made the lab work much more enjoyable.

Thank you to Assoc. Prof. Martyn Coles for his help with crystallography and for always making group meetings a pleasant experience, even when they were scheduled first thing in the morning. I would also like to thank the rest of the inorganic synthesis group, Mat, Cara, Putri, Loc, Dylan, Ryan, Struan and Amanda for making the lab an enjoyable place to work every day. Ryan deserves a special mention, as he taught me most of the practical synthetic chemistry I know and was always a useful sounding board for new ideas. To Dr. Matthias Lein, your assistance with the computational work was invaluable.

I would also like to acknowledge VUW and the Curtis-Gordon scholarship for providing me with funding over the two years.

Thanks to all my flatmates over the two years, who have had to put up with my constant facts about both chemistry and science in general, without your support writing this thesis would have been a lot more difficult. I must also thank my parents, both for their backing and for not forcing me into getting a real job when I finished my undergraduate studies. Finally, I must pay thanks to my wife Emily. Without your love (and constant reminders to keep working) this thesis would not have been possible. 


\section{Contents}

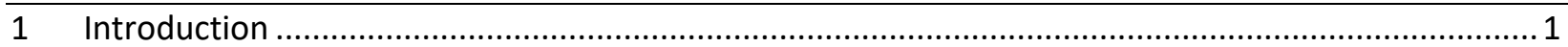

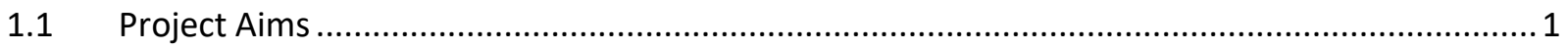

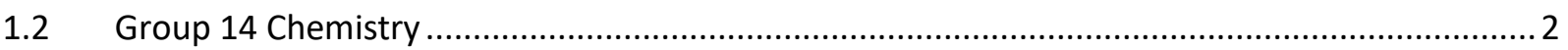

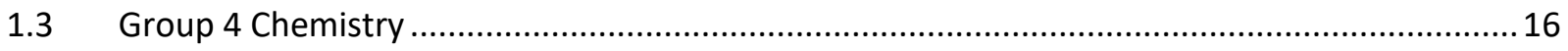

$1.4 \quad$ Imido-Bridged Bimetallic Compounds Synthesis and Reactivity .............................................24

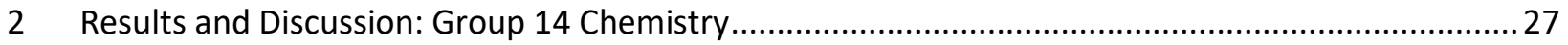

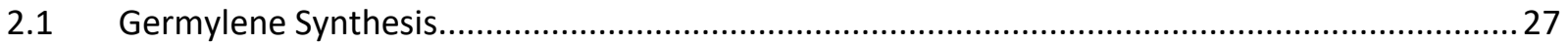

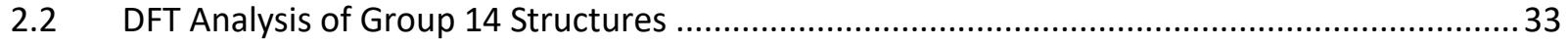

$2.3 \quad$ Mixed Coordination Germanium Species ........................................................................... 44

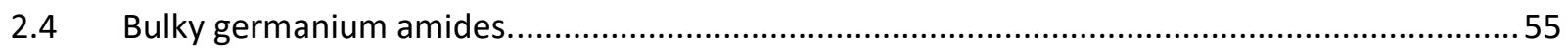

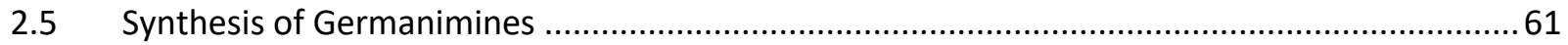

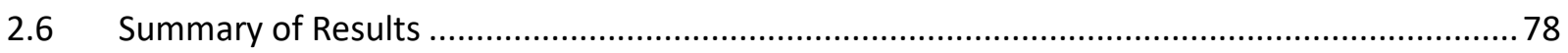

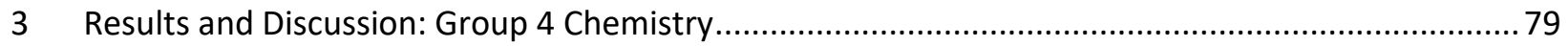

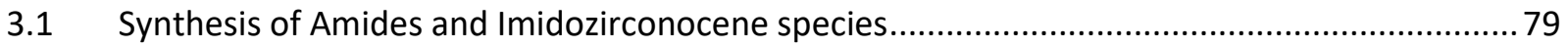

3.2 Insertion Reactions of Azides with alkylzirconocenes ..........................................................94

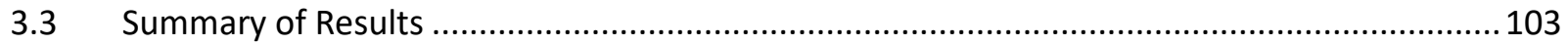

4 Results and Discussion: Group 4 - Group 14 Heterobimetallics .................................................104

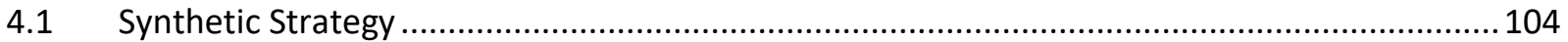

4.2 Reactions between Group 4 and Group 14 Compounds .................................................. 104

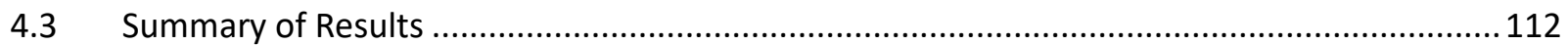

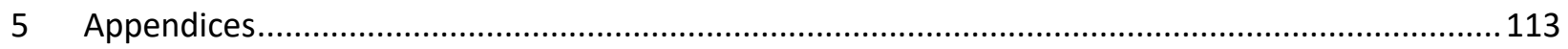

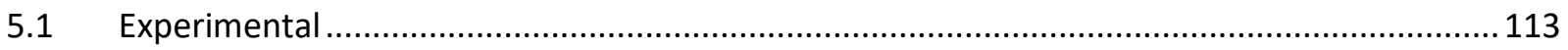

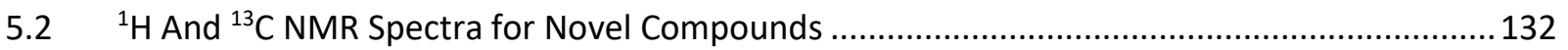

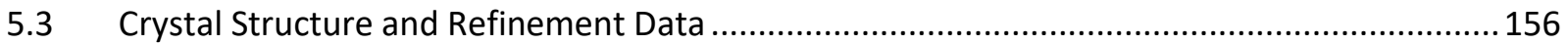

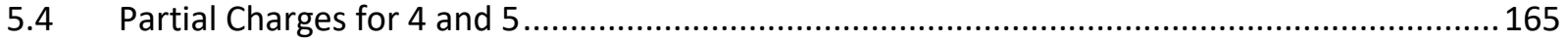

5.5 DFT Molecular Orbital Calculation Outputs for [Ge(HMDS) 2 ; I I......................................... 166

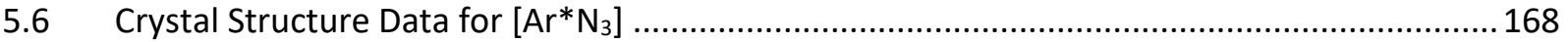

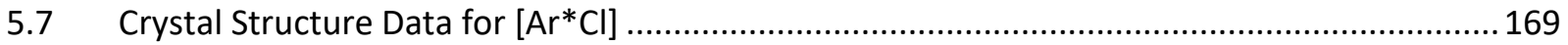

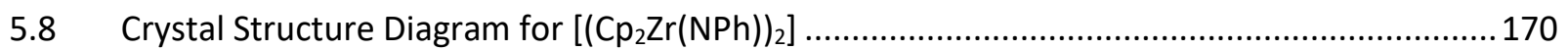

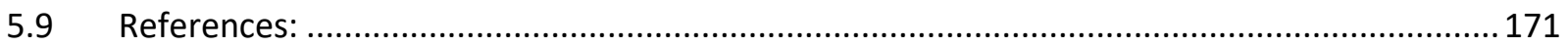




\section{List of Figures}

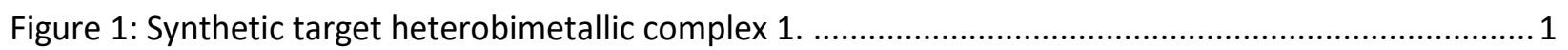

Figure 2: Electron occupation of singlet and triplet metallene. ............................................................ 3

Figure 3: Orbital energy diagram showing relative stabilisation and destabilsation of HOMO through

changes in the electronegativity of the $\alpha$ substituents. ....................................................................... 4

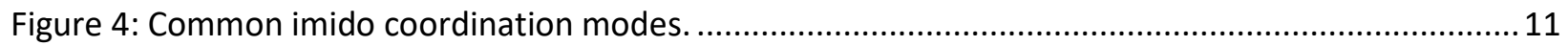

Figure 5: Germanes XXIII and XXIV, products of azide insertion into germanimine bond. ......................14

Figure 6: Leung's complex (XXV) and Jones's germanium dimer after hydrogen addition (XXVI)............14

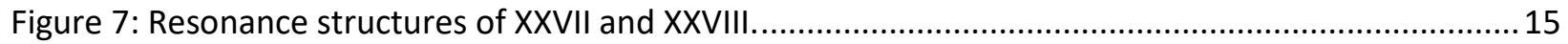

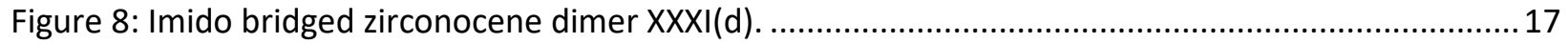

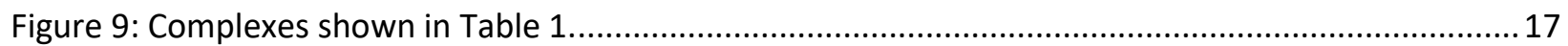

Figure 10: Relative orbital energy diagram of alkyl and aryl imidozirconocenes...................................19

Figure 11: Orbital energy diagram showing bonding interaction of the heterobimetallic complex XXVII

and relative stabilisation and destabilisation of the $\pi$ and $\pi^{*}$ orbitals. ..............................................24

Figure 12: Ortho, meta and para positions of 2

Figure 13: ORTEP representation of 2. 30\% Thermal ellipsoids. Hydrogens omitted for clarity...............29

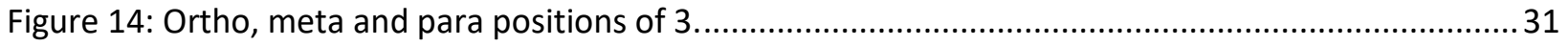

Figure 15: ORTEP representation of XL, showing out of the NCCCN plane tin atom. ............................. 31

Figure 16: ORTEP representation of 3. 30\% Thermal ellipsoids. Hydrogens omitted for clarity................32

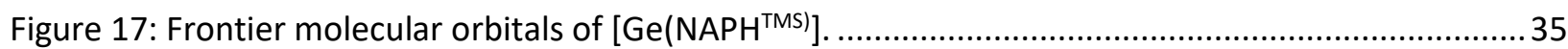

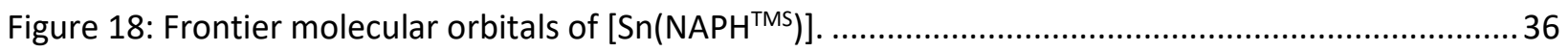

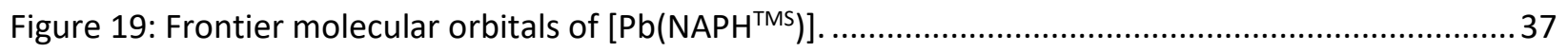

Figure 20: Diagram showing energy of frontier MO's for [NAPH ${ }^{\mathrm{TMS}}$ ] compounds...................................38

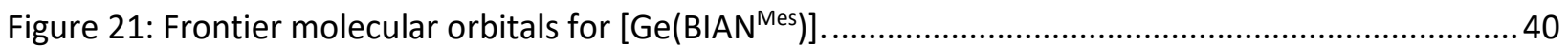

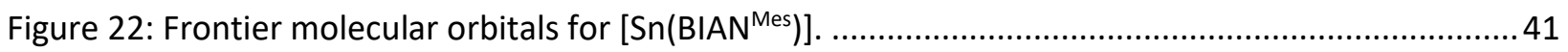

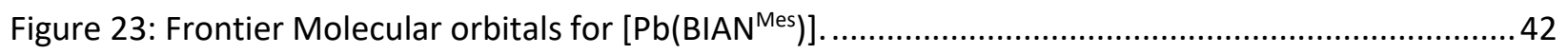

Figure 24: Diagram showing energy of frontier MO's for [BIAN ${ }^{\text {Mes }}$ ] compounds. ..................................43

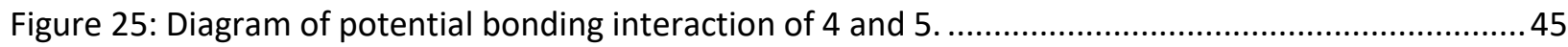

Figure 26: ORTEP representation of 4. 30\% Thermal ellipsoids. Hydrogens omitted for clarity...............46 
Figure 27: ORTEP representation of 5. 30\% Thermal ellipsoids. Hydrogens omitted for clarity.

Figure 28: Conformations of 4 and 5 as viewed down the Ge(1) Ge(2) bond for solid-state data of 4 (eclipsed), and 5 (staggered). .52

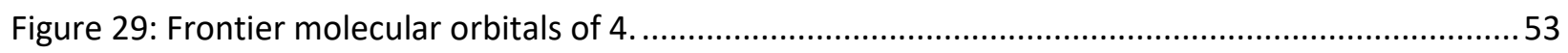

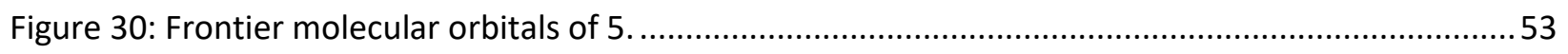

Figure 31: ORTEP representation showing connectivity of 6 . Hydrogens omitted for clarity...................56

Figure 32: Amido-bridged germanium compounds XLIII and XLIV .....................................................56

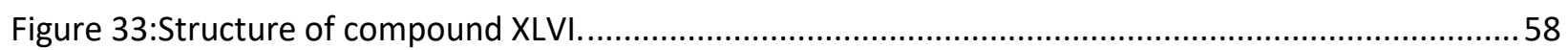

Figure 34: ORTEP representation of 7. 30\% Thermal ellipsoids. Hydrogens omitted for clarity...............60

Figure 35: ORTEP representation of 8. 30\% Thermal ellipsoids. Hydrogens omitted for clarity...............63

Figure 36: ORTEP representation of 9 (one molecule shown). 30\% Thermal ellipsoids. Hydrogens omitted for clarity. .65

Figure 37: ORTEP representation of 10. 30\% Thermal ellipsoids. Hydrogens omitted for clarity..............68

Figure 38: ORTEP representation of 11. 30\% Thermal ellipsoids. Hydrogens omitted for clarity..............71

Figure 39: ORTEP representation of 12. 30\% Thermal ellipsoids. Hydrogens omitted for clarity.............. 74

Figure 40: ORTEP representation of 13. 30\% Thermal ellipsoids. Hydrogens omitted for clarity..............77

Figure 41: ORTEP representation of 14. 30\% Thermal ellipsoids. Hydrogens omitted for clarity..............81

Figure 42: ORTEP representation of $15.30 \%$ Thermal ellipsoids. Hydrogens omitted for clarity..............83

Figure 43: ORTEP representation of 16. 30\% Thermal ellipsoids. Hydrogens omitted for clarity..............85

Figure 44: ORTEP representation of 17. 30\% Thermal ellipsoids. Hydrogens omitted for clarity.............87

Figure 45: ORTEP representation of 19. 30\% Thermal ellipsoids. Hydrogens omitted for clarity. Disorder

in the Cp2 ring modelled over two positions

Figure 46: ORTEP representation of $20.30 \%$ Thermal ellipsoids. Hydrogens omitted for clarity.

Cyclopentadienyl ligands shown with centroids.

Figure 47: ORTEP diagram of $21.30 \%$ thermal probability, hydrogens omitted for clarity.

Cyclopentadienyl ligands shown with centroids.

Figure 48: ORTEP diagram of 22. 30\% thermal probability, hydrogens omitted for clarity.

Cyclopentadienyl ligands shown with centroids. .98

Figure 49: ORTEP representation of 23. 30\% Thermal ellipsoids. Hydrogen atoms omitted for clarity. . 100 Figure 50: ORTEP diagram of 24 with 30\% thermal probability, hydrogens omitted for clarity.

Cyclopentadienyl ligands shown with centroids. 102 
Figure 51: Partial charges calculated for 4 showing negative (red) and positive (blue) partial atom

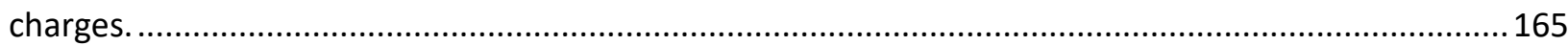

Figure 52: Partial charge calculation output of 5 showing negative (red) and positive (blue) partial atom

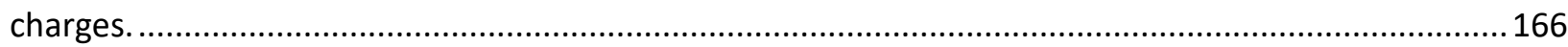

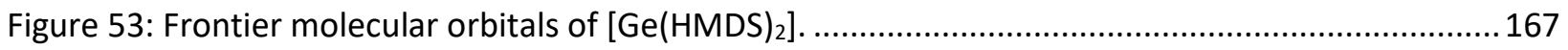

Figure 54: ORTEP representation of 25. 30\% Thermal ellipsoids. Hydrogens omitted for clarity............168

Figure 55: ORTEP representation of 26, 30\% thermal ellipsoids. Hydrogens omitted for clarity. ...........169

Figure 56: ORTEP diagram for $\left[\left(\mathrm{Cp}_{2} \mathrm{Zr}(\mathrm{NPh})\right)_{2}\right]$. Shown for connectivity purposes only. Isotropic model.170 


\section{List of Tables}

Table 1: Relevant bond distances and angles for a range of imidozirconocene complexes. ....................17

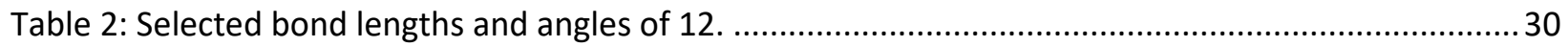

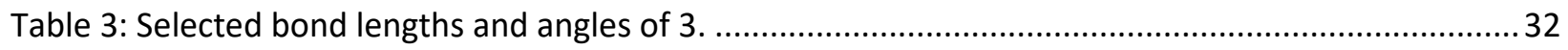

Table 4: Crystal structure bond lengths. Computational bond lengths in brackets. The NCN plane is defined as the plane of the nitrogen atom with the carbon atom $\beta$ to both nitrogen atoms. .................34

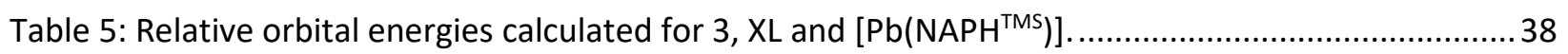

Table 6: Crystal structure of selected bond lengths compared to computationally derived optimized

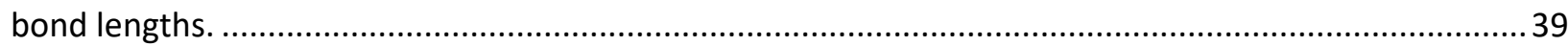

Table 7: Crystal structure of selected bond lengths compared to computationally derived optimised

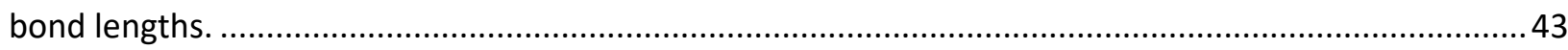

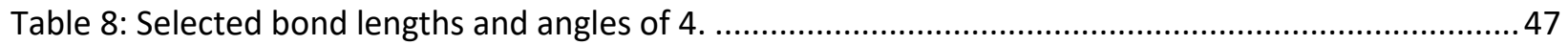

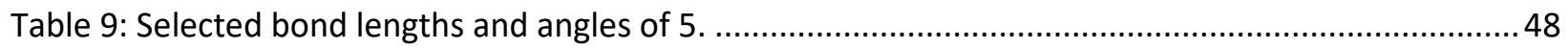

Table 10: Selected bond lengths and angles of 4 and 5, calculated quantities shown in brackets............51

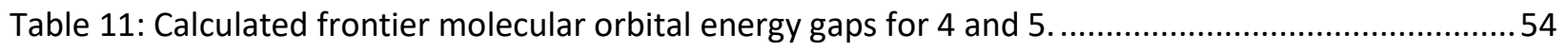

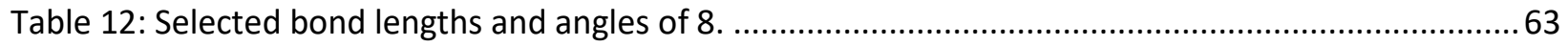

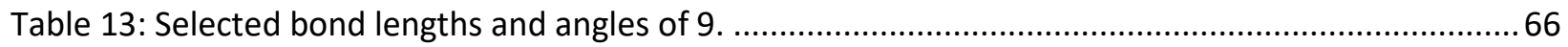

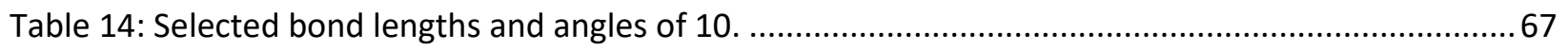

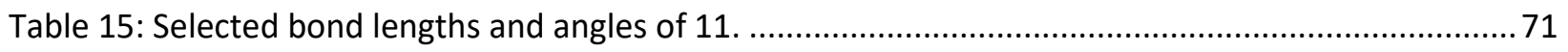

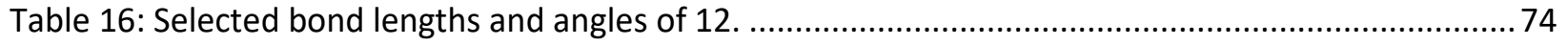

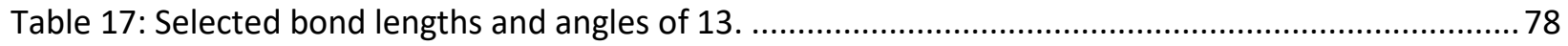

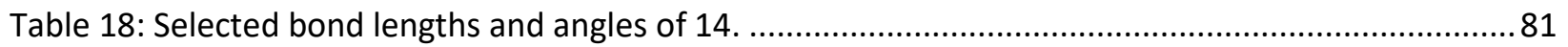

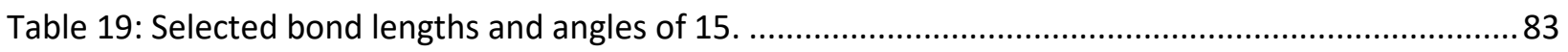

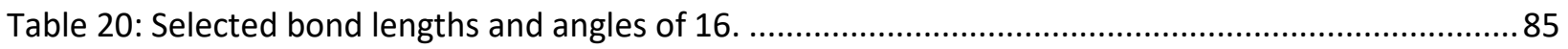

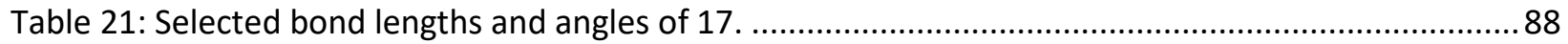

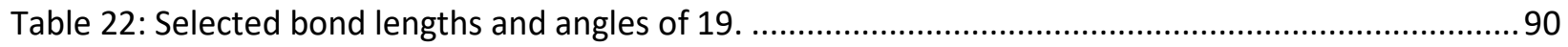

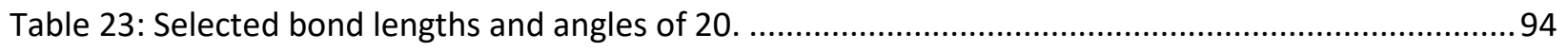

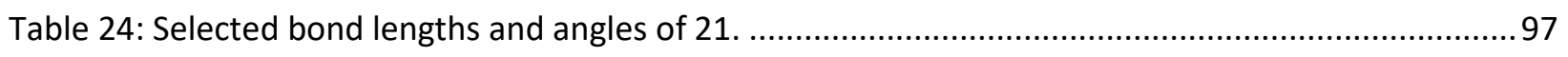

Table 25: Selected bond lengths and angles of 22.

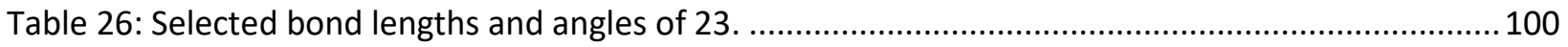


Table 27: Selected bond lengths and angles of 24. .102

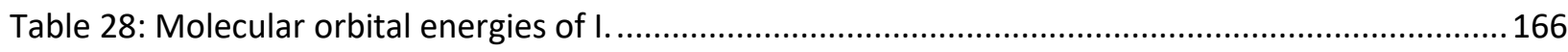

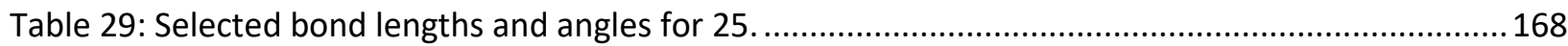

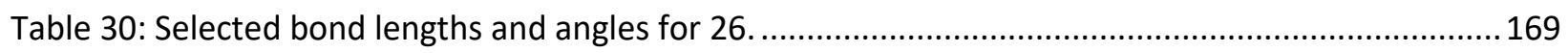




\section{List of Schemes}

Scheme 1: Proposed synthetic routes to the desired heterobimetallic complex 1 ................................. 1

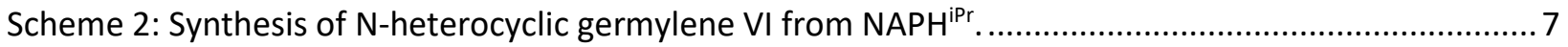

Scheme 3: Synthesis of digermene and germylene by salt metathesis.................................................. 9

Scheme 4: Intermolecular $\mathrm{N}-\mathrm{H}$ and $\mathrm{H}-\mathrm{H}$ activation by germylene compound. ......................................10

Scheme 5: Synthesis of Wesemann's tetrylene complex using adamantyl azide. ..................................13

Scheme 6: Synthesis of imidozirconocene XXX through thermolysis to eliminate methane....................16

Scheme 7: Reactions of imidozirconocenes with various heterocumulenes. ..........................................19

Scheme 8: Catalytic cycle of imidozirconocenes mediated imide metathesis. .......................................20

Scheme 9: Catalytic cycle of imidozirconocene mediated alkyne hydroamination. ...............................2 21

Scheme 10: Synthesis and reactivity of tetrazene zirconium complexes from an imidozirconocene........21

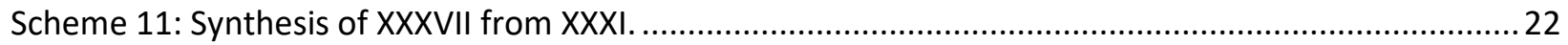

Scheme 12: Synthesis of heterobimetallic XXXIX via the Negishi reagent. ............................................25

Scheme 13: Reactivity of complex XXXIX with a range of polar small molecules....................................26

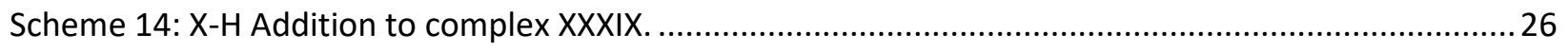

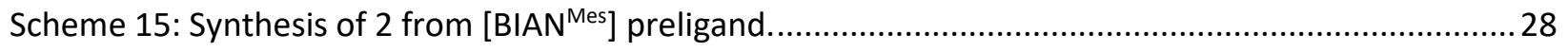

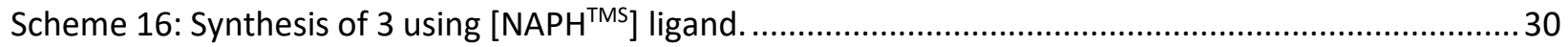

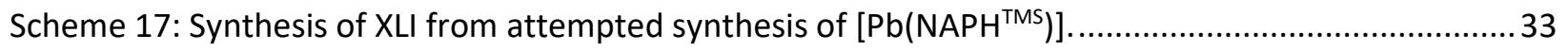

Scheme 18: Reaction of bulky lithiated amines with germanium dichloride. ..........................................55

Scheme 19: Lappert's different proposed synthetic pathways to XLIII. .................................................57

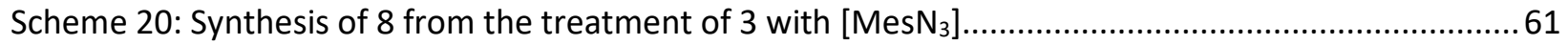

Scheme 21: Synthesis of 11 showing assumed intermediate in brackets. ...........................................69

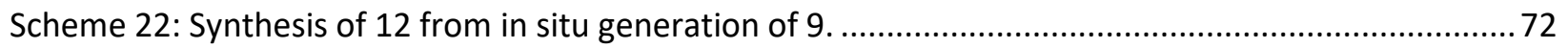

Scheme 23: Proposed reaction pathway for the synthesis of 12 showing a possible transition state......73

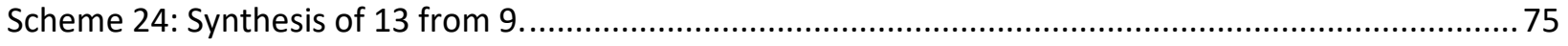

Scheme 25: Synthetic route to imidozirconocenes via salt methathesis. ............................................. 79

Scheme 26: Synthesis of 16 from transamination and salt metathesis reactions................................... 84

Scheme 27: Synthesis of 16 from transamination and salt metathesis reactions................................... 88

Scheme 28: Proposed synthetic route to imidozirconocenes from oxidative addition to the Negishi

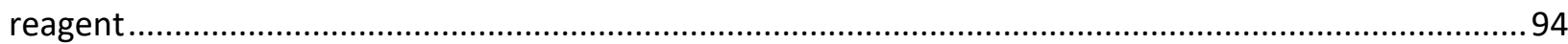




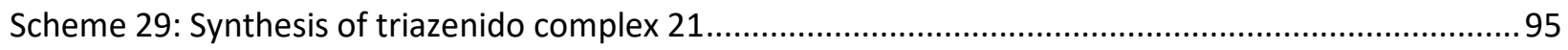

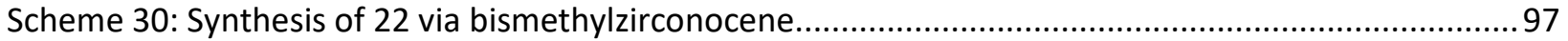

Scheme 31: Proposed Synthetic Route to heterobimetallic 1............................................................104

Scheme 32: Proposed reaction to amide bridged heterobimetallic 1 ................................................. 105

Scheme 33: Possible reaction of 15 to zirconium tetracycle............................................................. 108

Scheme 34: Reaction of Bisbenzylzirconcene with 2,6-dimethylaniline and [Ge(HMDS) $\left.{ }_{2}\right] \ldots \ldots \ldots \ldots \ldots \ldots \ldots . . . . . . .109$

Scheme 35: Possible products of the reaction between phenylamide-benzylzirconocene and

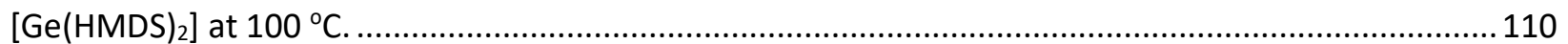

Scheme 36: Addition of germanimine 9 to the Negishi reagent. .......................................................... 111 


\section{List of Equations}

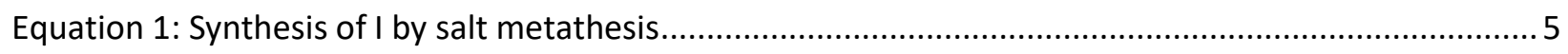

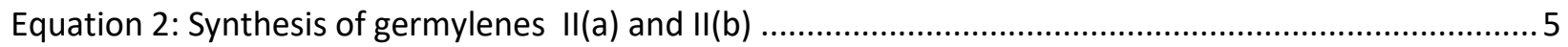

Equation 3: Synthesis of III(a), III(b) III(c) and III(d) via transamination reaction ..................................... 6

Equation 4: Synthesis of IV(a), IV(b) and IV(c) via transmetallation reaction........................................ 6

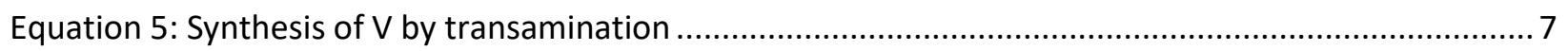

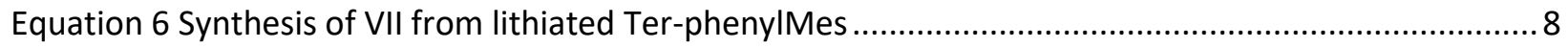

Equation 7: Synthesis of digermene from photolysis of cyclotrigermane ............................................... 8

Equation 8: Synthesis of XIII from boryl supported germylene XII ....................................................... 9

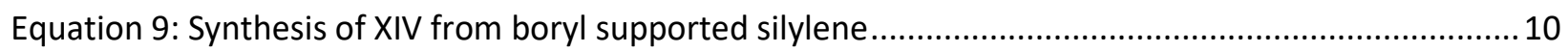

Equation 10 Activation of ammonia gas to give complex XVIII ..........................................................11

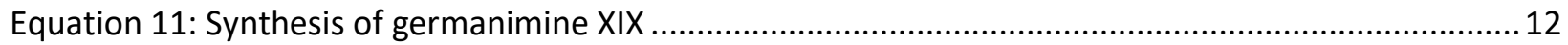

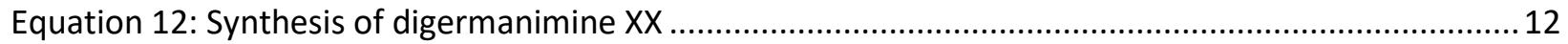

Equation 13: Cycloaddition of an azide with germanimine ............................................................ 13

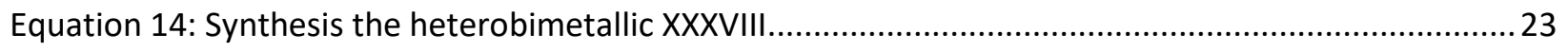

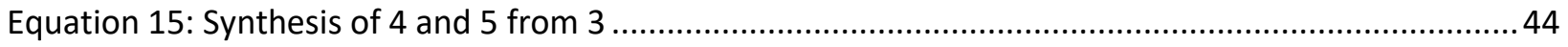

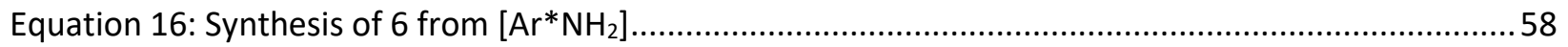

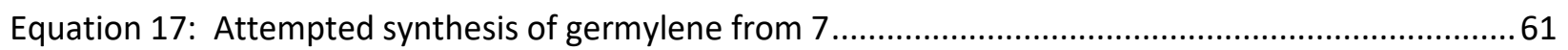

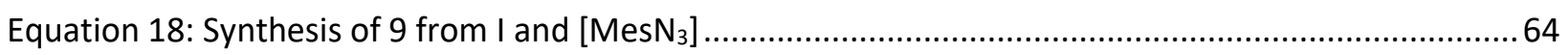

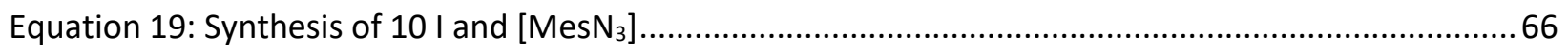

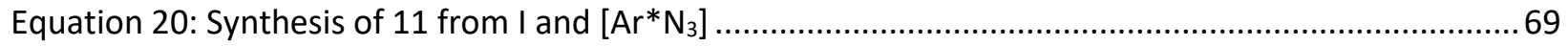

Equation 21: Synthesis of imidozirconocene via thermolysis of amidonzirconocene.............................79

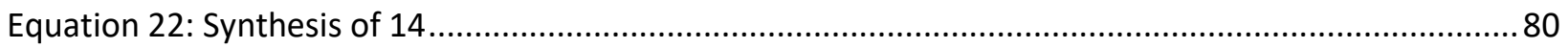

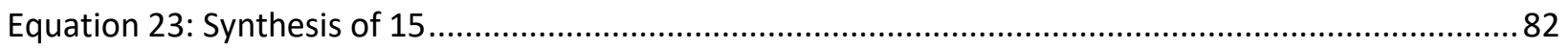

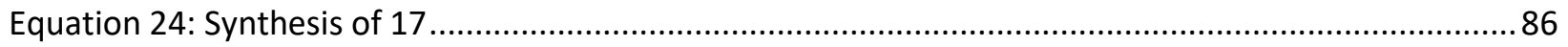

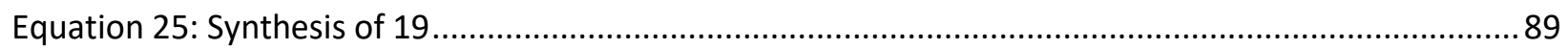

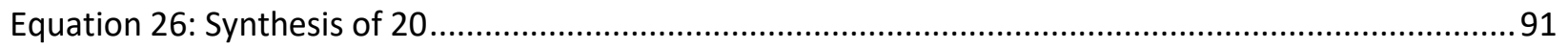

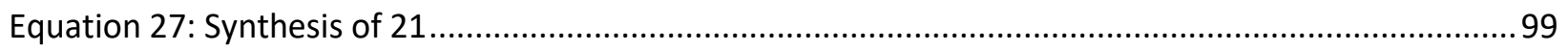

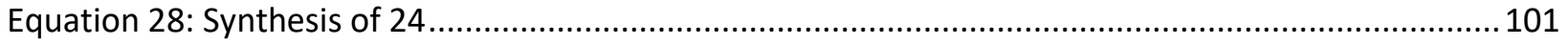

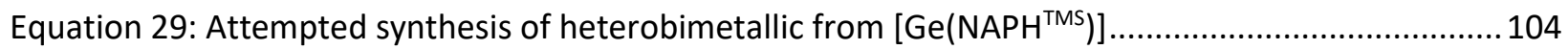


Equation 30: Attempted reaction of 15 and 2 to form heterobimetallic species 1 ..............................106

Equation 31: Attempted synthesis of heterobimetallic 1 from 15 and 2 .........................................107

Equation 32: Attempted Synthesis of desired heterobimetallic from I and 16 ...................................109

Equation 33: Proposed reduction of zirconocene dichloride to form heterobimetallic ....................... 110

Equation 34: Reduction of Zirconocene dichloride with magnesium followed by treatment with 9 ......111

Equation 35: Attempted synthesis of heterobimetallic from reduction of zirconocene dichloride.........112 


\section{Glossary}

$\delta$

$\lambda$

$\lambda_{\max }$

NMR

${ }^{1} \mathrm{H}$ NMR

${ }^{13}$ C NMR

$\alpha$

$\AA$

Ad

Ar

Ar (characterisation)

Approx.

$\beta$

Bn

br

$\mathrm{Bu}$

$\mathrm{C}_{6} \mathrm{D}_{6}$

Cp

Cp*

Cy

d

dd

DHA

Dipp

DMP

eq

Eq.

Et

$\mathrm{Et}_{2} \mathrm{O}$

${ }^{i} \mathrm{Pr}$
Chemical shift (ppm)

Wavelength

Wavelength of maximum absorption

Nuclear magnetic resonance

Proton nuclear magnetic resonance

Carbon nuclear magnetic resonance

Alpha-position (adjacent atom)

Angstrom

Adamantyl

Some form of aryl substituent

Aryl or aromatic region

Approximately

Beta-position (1 atom removed from adjacent atom)

Benzyl group

Broad signal

$n$-butyl substituent

Deuterated benzene

Cyclopentadienyl ring

Pentamethylcyclopentadienyl ring

Cyclohexyl

Doublet

Doublet of doublets

Dihedral angle

2,6-Diisopropylphenyl

2,6-Dimethylphenyl

Equation

Equivalence

Ethyl

Diethyl ether

Iso-propyl substituent 


\begin{tabular}{|c|c|}
\hline HMDS & Hexamethyldisilazide \\
\hline HOMO & Highest occupied molecular orbital \\
\hline IR & Infra-red spectroscopy \\
\hline J & Scalar coupling constant $(\mathrm{Hz})$ \\
\hline LUMO & Lowest unoccupied molecular orbital \\
\hline m & Multiplet \\
\hline meta & Meta- position \\
\hline Me & Methyl \\
\hline Methine & $\mathrm{A} \mathrm{CH}$ group \\
\hline Mes & Mesityl group \\
\hline Methylene & $\mathrm{A} \mathrm{CH}_{2}$ group \\
\hline ortho & Ortho- position \\
\hline para & Para- position \\
\hline $\mathbf{P h}$ & Phenyl \\
\hline ppm & Parts per million \\
\hline $\mathbf{R}$ & An unspecified substituent \\
\hline $\mathbf{s}$ & Singlet \\
\hline $\mathbf{t}$ & Triplet \\
\hline $\mathbf{q}$ & Quartet \\
\hline quin & Quintet \\
\hline tol & Toluene \\
\hline THF & Tetrahydrofuran \\
\hline${ }^{t} \mathrm{Bu}$ & Tert-butyl substituent \\
\hline TMS & Trimethylsilyl \\
\hline UV/vis & Ultraviolet-visible spectroscopy \\
\hline $\mathbf{v}$ & Wavenumbers $\left(\mathrm{cm}^{-1}\right)$ \\
\hline NCCCN & Refers to the plane defined by $N_{1}-C_{1}-C_{2}-C_{3}-N_{2}$ \\
\hline
\end{tabular}




\section{List of newly synthesised compounds}

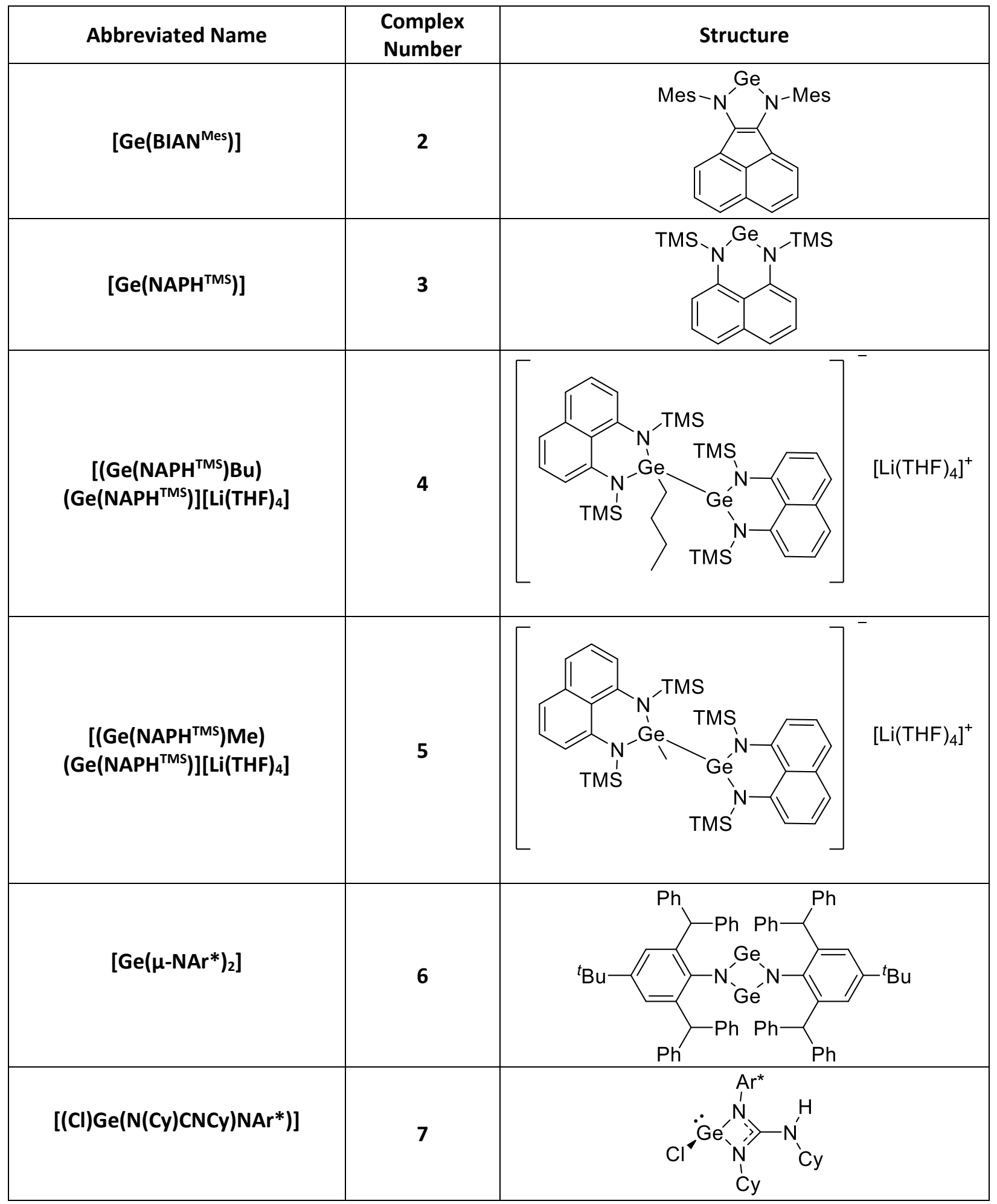




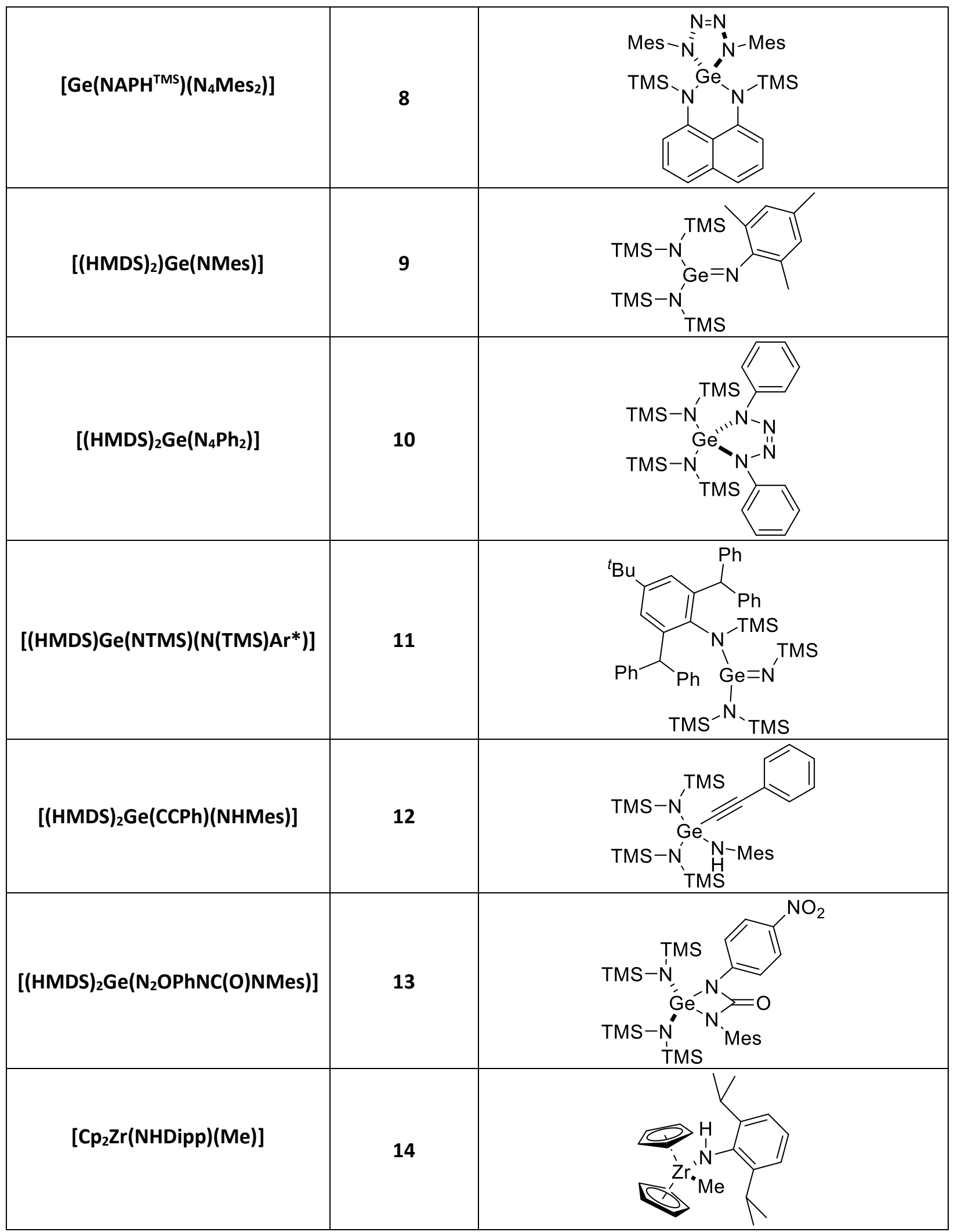




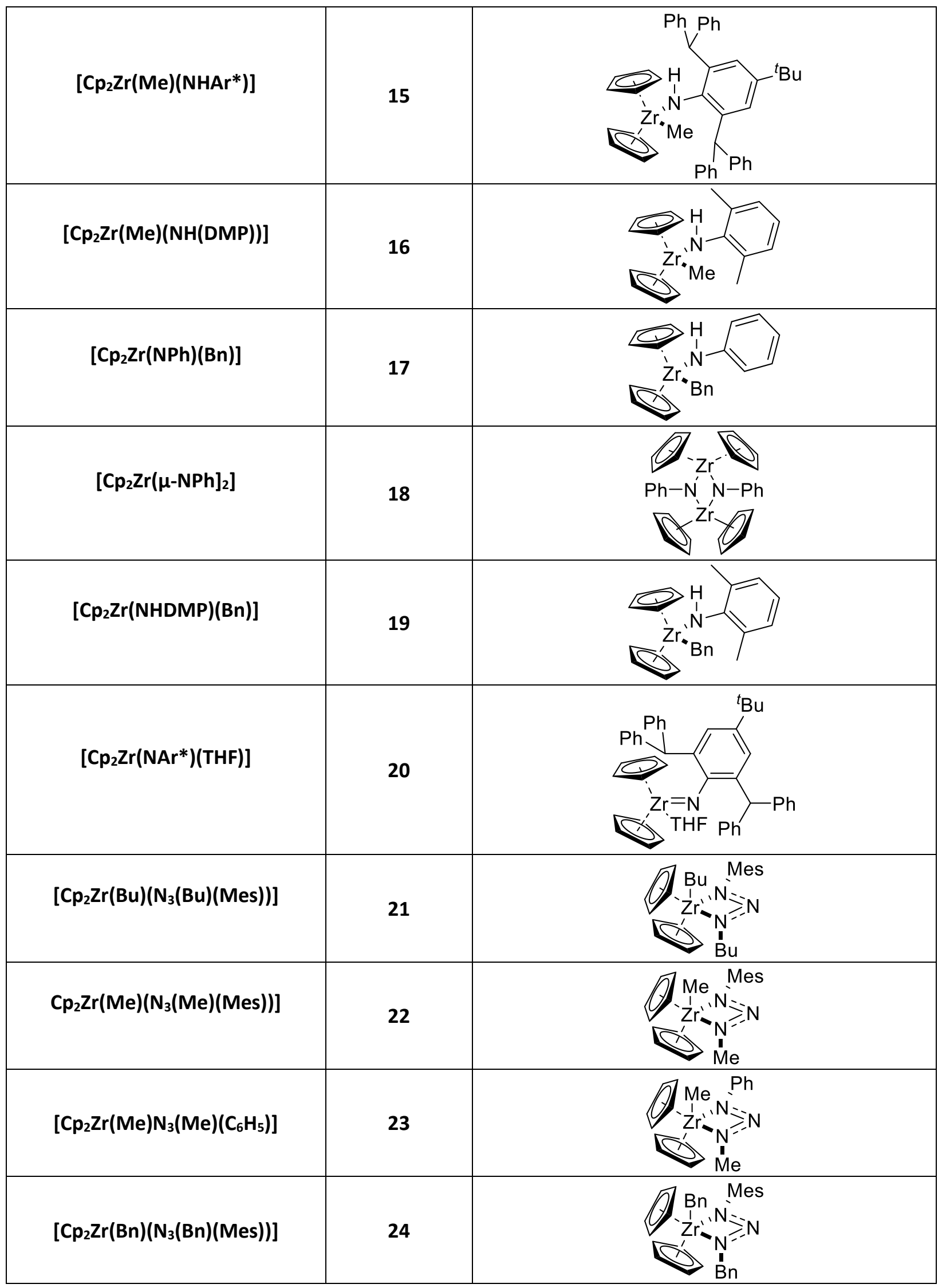




\begin{tabular}{|c|c|c|c|}
\hline & 25 & & \\
{$[\mathrm{Ar} * \mathrm{N3}]$} & 26 & &
\end{tabular}




\section{Introduction}

\subsection{Project Aims}

The aim of this project was to synthesise an amide supported germanium - zirconium heterobimetallic complex 1, similar to known transition metal heterobimetallic complexes. ${ }^{1}$

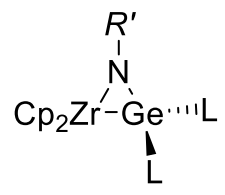

1

Figure 1: Synthetic target heterobimetallic complex 1.

The desired complex was targeted due to its possible unique reactivity with small molecules due to having a highly polarised zirconium germanium bond. The activation of small molecules by metal complexes has traditionally been an area of research almost exclusively reserved for transition metals. However, the development of sterically demanding ligand systems that can kinetically stabilise reactive low valent main group complexes has increased the number of oxidation states commonly accessible to main group compounds. This kinetic stabilisation has led to an increase in the known chemistry of main group complexes including the synthesis of complexes with multiple bonds between the heavier main group elements, synthesis of stable low valent compounds with open coordination sites, complexes with stable paramagnetic electron configurations (radicals) with unpaired electrons centred on heavier main group elements and the development of main group complexes with frustrated Lewis pairs. ${ }^{2-5}$ Many of these main group compounds have been shown to activate a range of both polar and non-polar bonds of small molecules including $\mathrm{H}-\mathrm{H}, \mathrm{N}-\mathrm{H}, \mathrm{C}-\mathrm{H}, \mathrm{B}-\mathrm{H}$ and $\mathrm{Si}-\mathrm{H}$ bonds. ${ }^{6-8}$

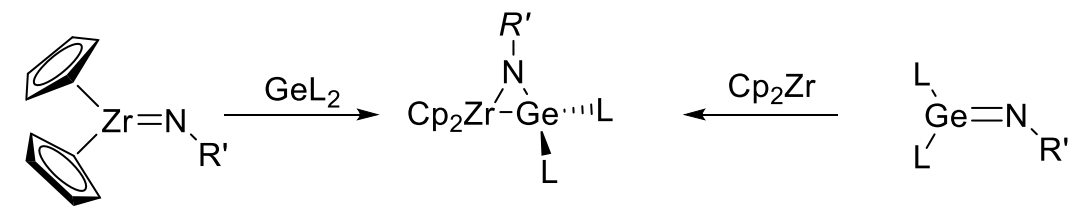

1

Scheme 1: Proposed synthetic routes to the desired heterobimetallic complex 1. 
Two approaches were proposed for the synthesis of $\mathbf{1}$. The first was from the reaction of a germylene with an imidozirconocene, the second was from the reaction of a $\mathrm{Zr}(\mathrm{II})$ species (the Negishi reagent ' $\mathrm{Cp}_{2} \mathrm{Zr}$ ') with a germanimine (Scheme 1). ${ }^{9}$ This thesis contains the theory, synthesis and characterisation of a range of germanium and zirconium compounds used in the attempted synthesis of 1 . All compounds and precursors synthesised in this project (with the exception of zirconocene dichloride, phenylazide, meistylazide, 25 and 26) were air and moisture sensitive, as such, all manipulations were performed under a dry and oxygen free $\mathrm{N}_{2}$ environment using Schlenk-line and cannula techniques, or in a nitrogen filled glovebox.

\subsection{Group 14 Chemistry}

\subsubsection{Metallene multiplicity and electronic stabilisation}

Group 14 metallenes are main group $\mathrm{MX}_{2}(\mathrm{M}=\mathrm{Si}, \mathrm{Ge}, \mathrm{Sn}, \mathrm{Pb})$ species with $\mathrm{M}$ in the +2 oxidation state and a lone pair of electrons. They are the heavier analogues of carbenes and can undergo similar chemistry to their carbon analogues. ${ }^{10}$ The lighter metallenes $(\mathrm{M}=\mathrm{Si}, \mathrm{Ge})$ possess a nucleophilic sp ${ }^{2}$-hybrid $\sigma$-orbital, and an empty electrophilic $p$ orbital (the $p_{\pi}$ orbital). Heavier group 14 metallenes $(M=S n, P b)$ display different reactivity to the lighter analogues due to an increasing inert pair effect. This results in the valence electrons being held more tightly to the nucleus (due to ineffective shielding by $d$ and $f$ orbitals) and therefore do not participate in $\sigma$ bonding interactions. ${ }^{11-12}$ Due to this difference in reactivity, the heavier metallenes were not investigated in this project. In addition, as silylenes are less stable than germylenes, the main focus on creating a group 4-group 14 heterobimetallic complex was using germylenes. ${ }^{13}$ These complexes can exist as $\mathrm{GeX}_{2}$ species ( $\mathrm{X}=$ anionic ligand), however will readily dimerise if not kinetically stabilised using significant steric bulk on the anionic $X$ ligand.

Group 14 metallenes can exist in either a singlet or triplet multiplicity state. The singlet state occurs when both electrons of the lone pair occupy the $\sigma$ symmetry orbital (the highest occupied molecular orbital 


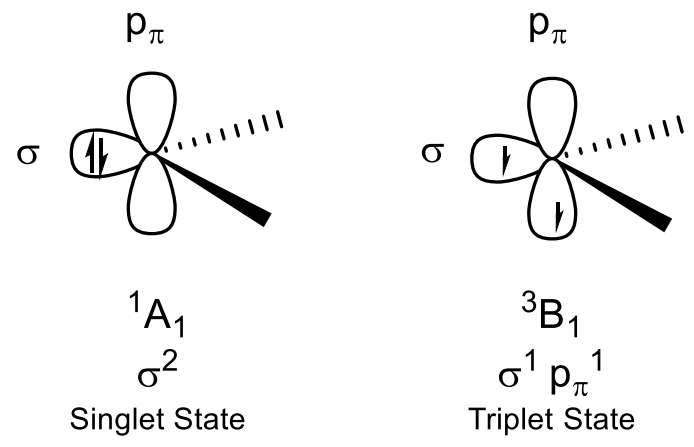

Figure 2: Electron occupation of singlet and triplet metallene.

(HOMO)), leaving an empty $\mathrm{p}_{\pi}$ orbital (the lowest unoccupied molecular orbital (LUMO)), while the triplet state occurs when one electron is located in each of these orbitals (Figure 2).

The energy splitting of these orbitals generally dictates the ground state of the metallene, with high $\sigma-p_{\pi}$ orbital gaps favouring the singlet ground state, and small $\sigma-p_{\pi}$ energy splitting favouring the triplet ground state. The ground state of the metallene can have a large effect on the reactivity of the species, as singlet metallenes will have greater amphiphilic reactivity (acting as a Lewis acid or Lewis base), while triplet ground state metallenes react with greater radical character. The singlet state becomes more dominant moving down the group 14 metallenes, due to the increased $\sigma-p_{\pi}$ orbital energy splitting, as the $\sigma$ orbital increases in s-character down the group. ${ }^{14}$

The reactivity of carbenes and their group 14 metalllene analogues $\left(\mathrm{X}_{2} \mathrm{M}:\right)$ towards bond activation has been known to be highly dependent on the nature of the $\mathrm{X}$ substituent due to its effect on the HOMOLUMO gap of the molecule. A smaller HOMO-LUMO energy gap increases the carbene's reactivity towards bond activation. For instance, it has been shown that a narrower bond angle at the group 14 centre (X-M$\mathrm{X}$ ) is associated with a greater s-character of the HOMO orbital, a lower HOMO energy and hence a larger HOMO-LUMO energy gap (Figure 3). ${ }^{15-16}$ This increased s-character is usually found in molecules with a cyclic backbone or with extremely electron withdrawing $\alpha$ atoms of the $X$ substituents.

The LUMO energy can also be altered through the use of other substituents. For instance, $\pi$-donor substituents have been shown to make the LUMO molecular orbital to take on $\mathrm{M}-\mathrm{X} \pi^{*}$ character, increasing the relative energy of the orbital, thus increasing the HOMO-LUMO energy gap. This occurs because the donation of electron density into the $\mathrm{p}_{\pi}{ }^{*}$ orbital through increases its antibonding character, increasing the orbitals relative energy, resulting in carbene analogues with more $\pi$-donating ability usually existing in a singlet state due to the relative increase in the energy of the LUMO orbital. ${ }^{17}$ These strong $\pi$ donor substituents, for instance nitrogen, also normally decrease the X-M-X bond angle. DFT (density 
functional theory) calculations have provided data that indicates germylenes with $\alpha$ substituents with weak $\pi$ donor properties and high electropositivity have smaller HOMO-LUMO gaps and are therefore more kinetically labile to oxidative bond activation. ${ }^{16}$ As such germylenes with silyl and boryl $\alpha$ substituents generally exhibit higher reactivity than germylenes with nitrogen or phosphorus $\alpha$ substituents.

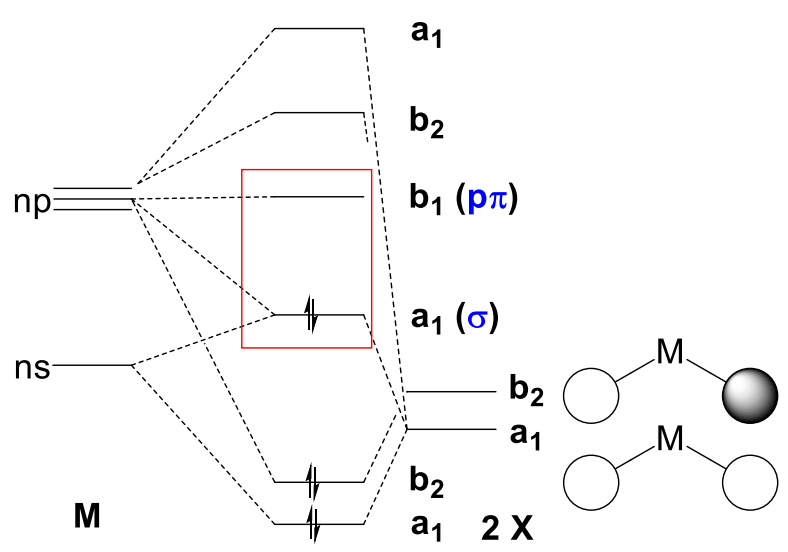

$a_{1}(\sigma)$ HOMO stabilised with increasing electronegativity.

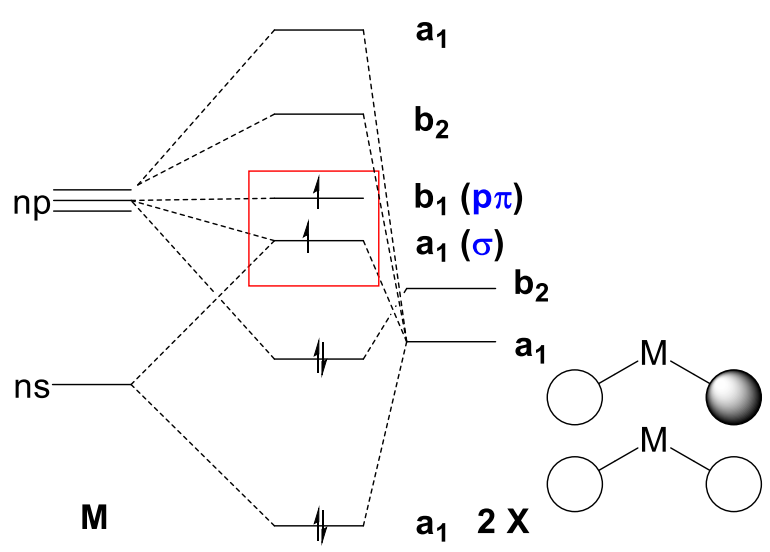

\section{Figure 3: Orbital energy diagram showing relative stabilisation and destabilsation of HOMO through} changes in the electronegativity of the $\alpha$ substituents.

The $\mathrm{X}-\mathrm{M}-\mathrm{X}$ bond angle plays an important role in the formation of singlet and triplet metallenes, with smaller bond angles $\left(<90^{\circ}\right)$ favouring singlet species while larger angles favour triplet species, as at angles below $90^{\circ}$ the energy of the singlet species drops below that of the triplet state. ${ }^{18}$ Bulky substituents that favour wide $\mathrm{X}-\mathrm{M}-\mathrm{X}$ bond angles therefore are more likely to adopt triplet multiplicity

\subsubsection{The Synthesis of Germylenes Supported with Acyclic Nitrogen based ligands and $\mathbf{N}$-Heterocyclic based ligands}

Germylenes that have nitrogen $\alpha$ substituents are synthesised through two standard routes, a salt metathesis reaction with a suitably lithiated pre-ligand or transamination to give the desired compound and a free amine. A lot of early work on stable monomeric germylenes was carried out by Lappert et al., 
who synthesised the monomeric bivalent germylene using the bulky $\left[\left(\mathrm{N}\left(\mathrm{Si}\left(\mathrm{Me}_{3}\right)\right)_{2}\right)\right]^{-}(\mathrm{HMDS})$ ligand system by treatment of $\mathrm{GeCl}_{2}$.dioxane with $\mathrm{LiN}\left(\mathrm{SiMe}_{3}\right)$ to give $\left[\mathrm{Ge}\left(\mathrm{N}\left(\mathrm{Si}\left(\mathrm{Me}_{3}\right)\right)_{2}\right)_{2}\right], \mathbf{I}$ (eq. 1). ${ }^{19}$

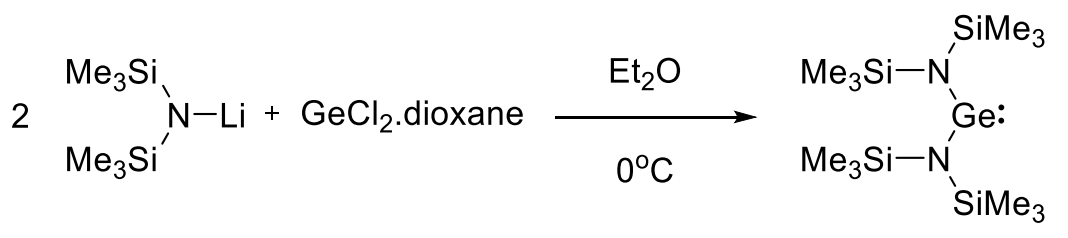

I

Compound I was a dimer in the solid state, but as a monomeric germylene species in solution as determined by absorption data indicating the presence of a lone pair on the germanium. The monomeric unit was shown to be a singlet :MR 2 species. ${ }^{20}$ Compound I has proven to be a useful precursor in the synthesis of $\mathrm{N}$-heterocyclic germylenes (NHGe's) by transamination due to the high proton affinity of (HMDS).

Bidentate ligands that form $N$-heterocycles upon coordination have also been used to stabilise monomeric germylenes. Ligands that coordinate through nitrogen atoms offer high stability as the nitrogen can act as both a $\sigma$-acceptor and $\pi$-donor and can hence electronically stabilise many compounds. The cyclisation of these ligands provides a chelating effect, which leads to them having increased stability relative to acyclic diaminogermylenes through more favourable entropy of formation. The bis(amido)silyl ligand $\left[{ }^{i} \operatorname{Pr}_{2} \operatorname{Si}(N R)_{2}\right]$ $\left(\mathrm{R}=\operatorname{Dipp}\left(2,6\right.\right.$-diisopropylphenyl), $\left.\mathrm{SiPh}_{3}\right)$ has been used to synthesise 2 stable germylenes (eq.2). ${ }^{5}$ Compound II(a) and II(b) were generated from the lithiated ligand and $\mathrm{GeCl}_{2}$.dioxane and shown to be thermally stable up to $300^{\circ} \mathrm{C}$

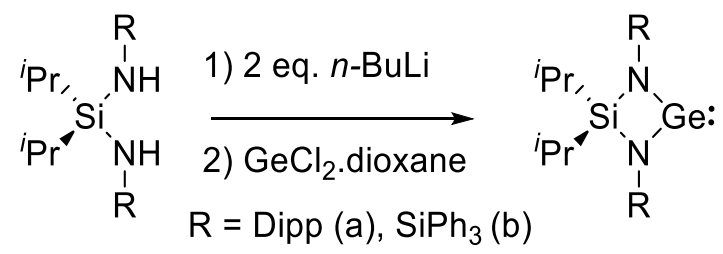

II(a), I(b)

Interesting solid-state data from the crystal structures of II(a) and II(b) reveal N-Ge-N bite angles of $81.26^{\circ}$ and $83.31^{\circ}$ respectively, as well as Ge-N bond lengths of $1.863 \AA$ for (a) and $1.883 \AA$ for (b). This is a relatively wide bite angle for an NHGe with a four-membered ring, indicating that the germanium atom is sitting deep into the bonding pocket of the ligand. The relatively short N-Ge bond distances of $1.863 \AA$ for 
II(a), relative to the normal range of Ge-N bond distance for nitrogen based ligands of 1.819-1.999 $\AA$ also indicates that the germanium atom sits further into the bonding pocket. ${ }^{13,21}$

The addition of 1,2 diaminobenzenes with one equivalent of the germylene I gives the benzannulated $\mathrm{N}$ heterocyclic germylenes with various bulky substituents on the amine (equation 3). ${ }^{22-23}$ The solid state structure of III(d) shows shorter Ge-N bond lengths of 1.866 and $1.862 \AA$ and a N-Ge-N bite angle of $87.17^{\circ}$.

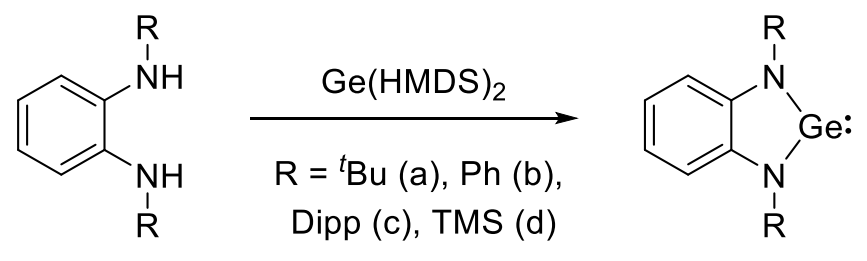

Germylenes with ligand systems bearing unsaturated backbones and ring fused annulated systems have aromatic resonance structures available to them. This aromaticity plays an important role in their electronic stability with both theoretical and experimental results showing that cyclic delocalisation of electrons promotes thermodynamic stabilisation. ${ }^{24}$

The BIAN ${ }^{\text {Dipp }}$ ligand IV(a) $\left(1,2-\left[\left(2,6-{ }^{i} \mathrm{Pr}_{2} \mathrm{C}_{6} \mathrm{H}_{3}\right) \mathrm{N}\right]_{2} \mathrm{C}_{12} \mathrm{H}_{6}\right), \operatorname{IV}(\mathbf{b}) \mathrm{BIAN}^{\text {bph }}\left(1,2-\left[\left(2-\mathrm{PhC}_{6} \mathrm{H}_{4}\right) \mathrm{N}_{2} \mathrm{C}_{12} \mathrm{H}_{6}\right)\right)$ and IV(c) $\operatorname{BIAN}^{\mathrm{dtb}}\left(1,2-\left[\left(2,5-{ }^{t} \mathrm{Bu}_{2} \mathrm{C}_{6} \mathrm{H}_{3}\right) \mathrm{N}\right]_{2} \mathrm{C}_{12} \mathrm{H}_{6}\right)$ have also been shown to stabilise monomeric germylene complexes using the $\sigma$ acceptor and $\pi$ donor properties associated with their nitrogen atoms (eq. 4). ${ }^{25}$
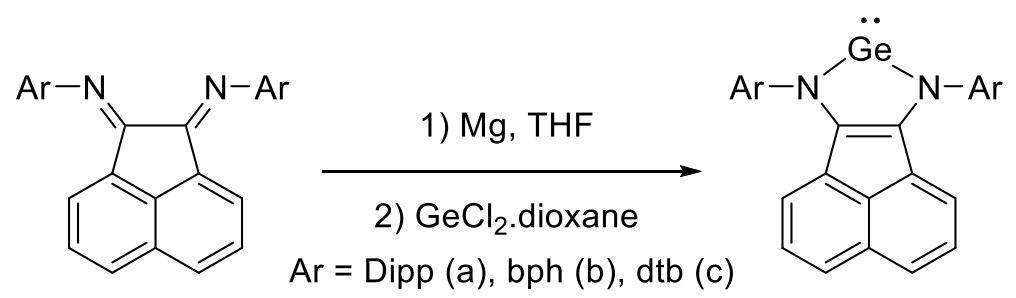

$\operatorname{IV}(a), \operatorname{IV}(b), \operatorname{IV}(c)$

The reduction of 1,2-bis[2,5-di-tert-butylphenyl)imido]acenaphthalene or other substituted acenaphthalene ligand with activated magnesium gives the monomeric magnesium complex. This compound can then undergo transmetallation with $\mathrm{GeCl}_{2}$. dioxane to give the complexes $\operatorname{IV}(\mathbf{a}), \operatorname{IV}(\mathbf{b})$ and IV(c). Complex IV(a) can also be synthesised from the reduction of $\mathrm{GeCl}_{4}$ with (BIAN ${ }^{\mathrm{Dipp}}$ ) Na. The solidstate crystal structures show average Ge-N bond lengths of $1.891 \AA$ for IV(a), 1.878 for IV(b) and $1.910 \AA$ for IV(c). The N-Ge-N bond angles of $85.20^{\circ}, 85.03^{\circ}$ and $84.01^{\circ}$ for IV(a), (b) and (c) respectively, indicate 
the steric bulk around the $\mathrm{N}$-aryl ring of the ligand, with the more sterically constraining $\mathrm{N}$-aryl groups resulting in a longer Ge-N bond length (IV(a) and IV(c)).

A similar ligand to the BIAN ligand that has also been used to synthesise monomeric germylenes is the (Z)N2,N3-dimesityl-but-2-ene-2,3-diamine ligand (mesityl = Mes $=2,4,6$ trimethylphenyl). Transamination of the preligand with I has led to compound $\mathbf{V}$ in a $41 \%$ yield (eq. 5). The Ge-N bond distance is $1.864 \AA$, and the $\mathrm{N}-\mathrm{Ge}-\mathrm{N}$ bond angle of $\mathbf{V}$ is $82.64^{\circ}$.

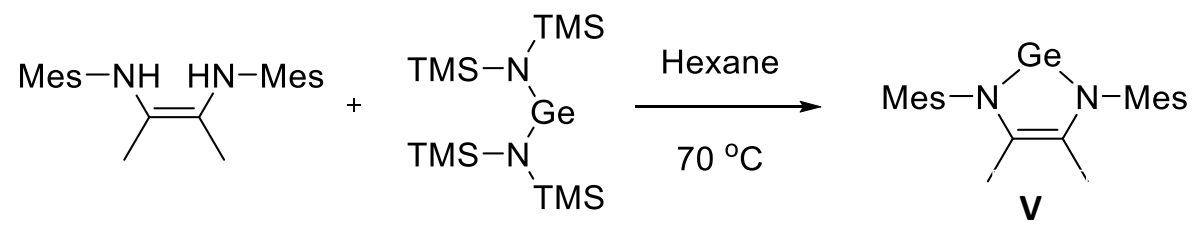

The ligand system 1,8-di(isopropylamino)naphthalene ( $\mathrm{NAPH}^{\mathrm{iPr}}$ ) has also been used to synthesise monomeric germylene compounds. ${ }^{26}$ The reaction of the lithiated ligand with $\mathrm{GeCl}_{2}$.dioxane gives $\mathbf{V I}$. The same compound can also be afforded by the reaction of the preligand with the germylene I (Scheme 2). The Ge-N bond lengths of VI are 1.841 and $1.842 \AA$ and the $\mathrm{N}-\mathrm{Ge}-\mathrm{N}$ bite angle is $97.32^{\circ}$. The increased bite angle of the ligand is due to the larger chelate of $\mathrm{Vl}$; creating a 6-memberd ring upon coordination of germanium, opposed to 5 or 4 membered rings in the previous examples. The solid-state structure of VI has the orientation of with both isopropyl groups sitting below the plane of the naphthalene-based backbone, with the germanium atom sitting slightly above the NCN plane (determined as the plane of the nitrogen atoms with the carbon atom in the $\beta$-position to both nitrogens).
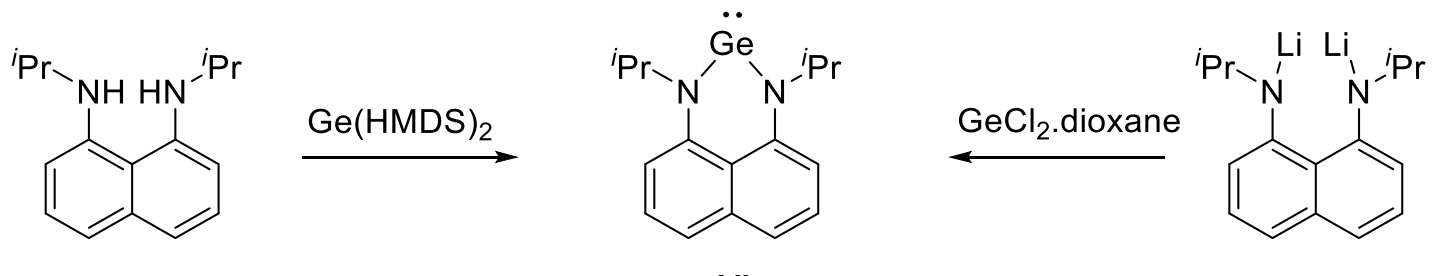

VI

Scheme 2: Synthesis of $\mathrm{N}$-heterocyclic germylene VI from NAPH ${ }^{i P r}$.

\subsubsection{The synthesis of germylenes supported other ligands}

Germylenes supported by non-nitrogen based ligands have also been synthesised. Carbon, oxygen and silicon based ligands are most common; however, others are known including phosphorus and boron 
based-ligands. ${ }^{16,27-30}$ To limit the scope of this project due to time constraints, only nitrogen based ligands were used in the attempted synthesis of 1. A full literature review is therefore outside the scope of this work, however other significant ligand systems used for the stabilisation of germylenes such as the terphenyl ligand will be discussed. Also included is an example highlighting the importance of sufficient steric bulk to kinetically stabilise monomeric germylene. For further information, the reader is directed to excellent reviews by Roesky et al. and Polo et al. ${ }^{31-32}$

The importance of steric bulk in kinetically stabilising germylenes is evident from the comparison of known monomeric germylenes to their digermene dimers. The bulky terphenyl ${ }^{\text {Mes }}$ based ligand has been shown to kinetically stabilise monomeric germanium(II) in both the solid and liquid state. ${ }^{33}$ The germylene [Ge $\left.\left(\mathrm{C}_{6} \mathrm{H}_{3}-2,5-\mathrm{Mes}_{2}\right)_{2}\right] ; \mathrm{VII}$, was synthesised from the addition of 2 equivalents of the lithiated ligand [ $\left.\mathrm{Li}\left(\mathrm{C}_{6} \mathrm{H}_{3}-2,5-\mathrm{Mes}_{2}\right)\right]$ to $\mathrm{GeCl}_{2}$.dioxane (eq. 6). The monoaryl derivative $\left[\mathrm{Ge}\left(\mathrm{C}_{6} \mathrm{H}_{3}-2,5-\mathrm{Mes}_{2}\right) \mathrm{Cl}\right]$ was also synthesised in the same manner using one equivalent of the lithiated ligand at $-78^{\circ} \mathrm{C}$.
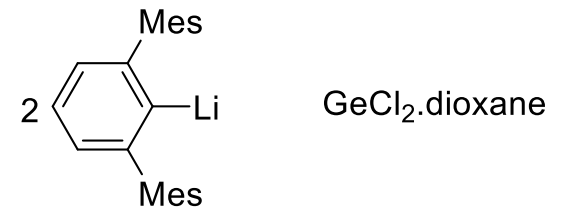
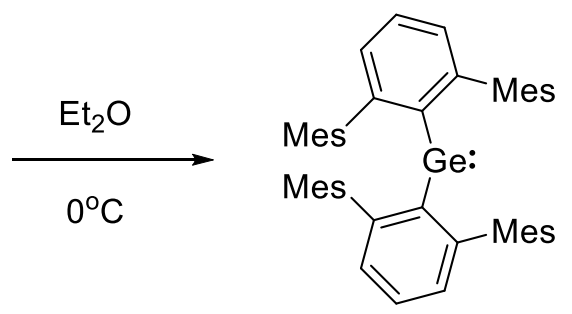

(6)

\section{VII}

The vital role of steric bulk in kinetic stabilisation of germylenes is highlighted by the compound tetrakis(2,6-diethylphenyl)digermene, IX(a). ${ }^{34}$ This species is formed from the photolysis of hexakis(2,6diethylphenyl)cyclotrigermane, VIII (eq. 7). The ligand 2,6-had insufficient steric bulk to kinetically stabilise the monomeric germylene, instead the digermene is formed.

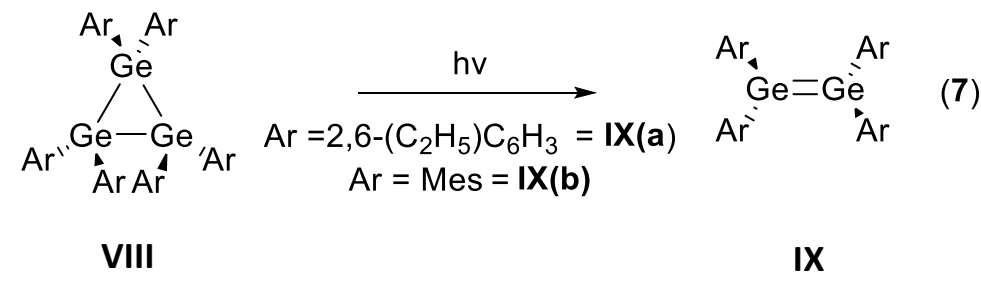

The isolation of tetramesityldigermene, $\mathbf{I X ( b )}$, also synthesised in the photolysis reaction from hexamesitylcyclogermane VIII, indicates that the mesityl ligand (2,4,6-trimethylphenyl) is also not bulky enough to kinetically stabilise a monomeric germylene (eq. 7$)^{35}$. 
The bulkier 2,4,6-triisopropylphenyl ligand has also been shown to be incapable of kinetically stabilising the germylene through steric bulk, instead giving the digermene $\mathbf{X I} .{ }^{36}$ However the 2,4,6-tert-butylphenyl ligand has been shown to kinetically stabilise the germylene in solution (Scheme 3) to give the monomeric germylene $\mathbf{X}$.

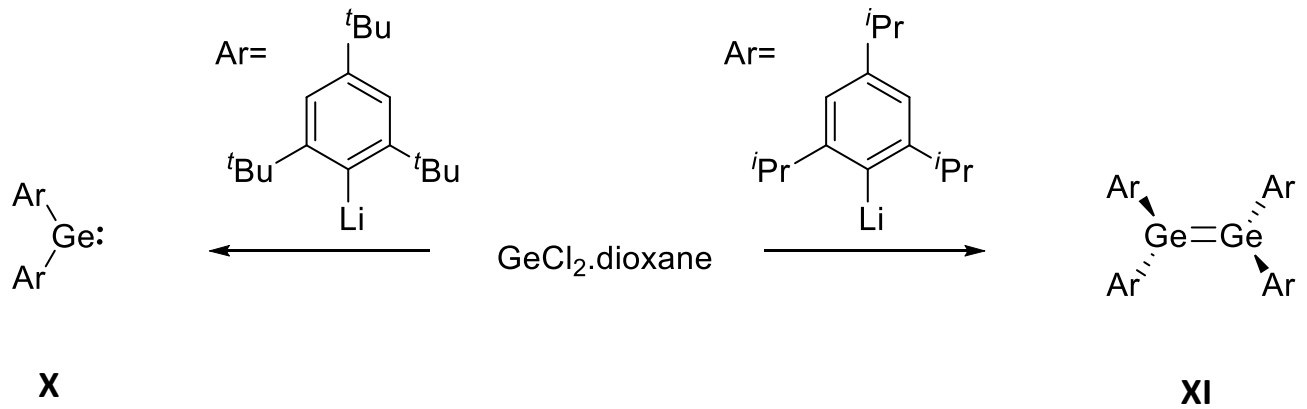

Scheme 3: Synthesis of digermene and germylene by salt metathesis.

\subsubsection{Germylene Reactivity}

The reactivity of germylenes has been studied for their potential use in the activation of small molecules. Variation of the HOMO-LUMO energy difference can have a large effect on the reactivity of the germylene towards a range of small molecules.

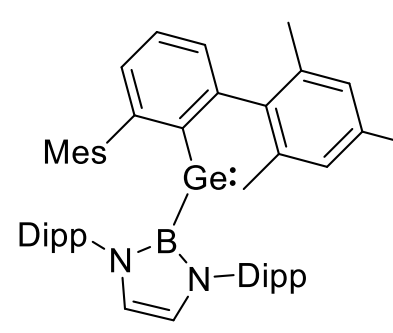

XII

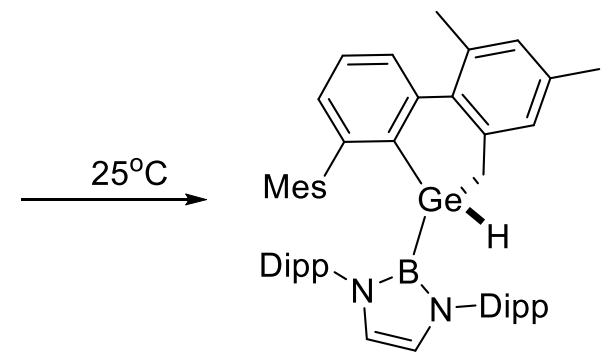

XIII

Aldridge et al. have showed that kinetically stabilised germylenes can undergo intramolecular $\mathrm{C}-\mathrm{H}$ activation at room temperature. ${ }^{16}$ Compound $\mathbf{X I I}$ undergoes intramolecular activation through cleavage of the $\mathrm{C}-\mathrm{H}$ bond on one of the ortho $\mathrm{CH}_{3}$ positions of the mesityl substituents, resulting in oxidation of the Ge(II) to Ge(IV) to give XIII (eq. 8). 
This $\mathrm{C}-\mathrm{H}$ bond activation can be rationalised by the extremely low HOMO-LUMO gap present in the germylene of $180 \mathrm{~kJ} \mathrm{~mol}^{-1}$ or $1.87 \mathrm{eV}$. Other metallene systems have also shown intramolecular $\mathrm{C}-\mathrm{H}$ activation. The acyclic silylene $\left[\left(\left(\mathrm{Me}_{3} \mathrm{Si}\right)(\mathrm{Dipp}) \mathrm{N}\right) \mathrm{Si}\left(\mathrm{B}(\mathrm{NDippCH})_{2}\right)\right]$ undergoes insertion into one of the $\mathrm{C}-\mathrm{H}$ bonds of the boryl-Dipp group to give XIV (eq. 9). ${ }^{8}$
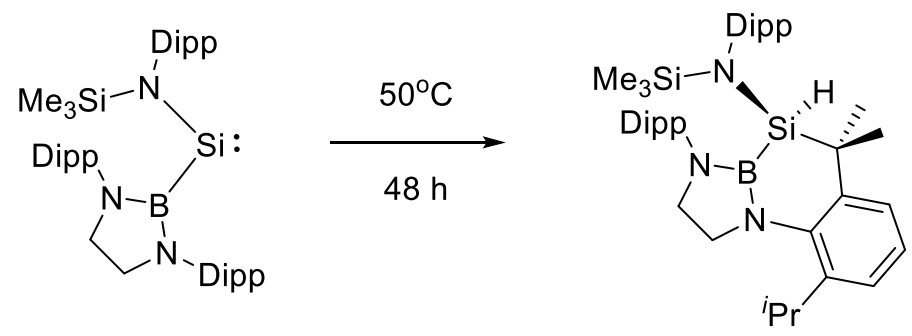

XIV

Germylenes can also activate a range of small molecules, as shown by the reaction of the germylene $\mathbf{X V}$ with ammonia and dihydrogen to give $\mathbf{X V I}$ and $\mathbf{X V I I}$ respectively (Scheme 4).

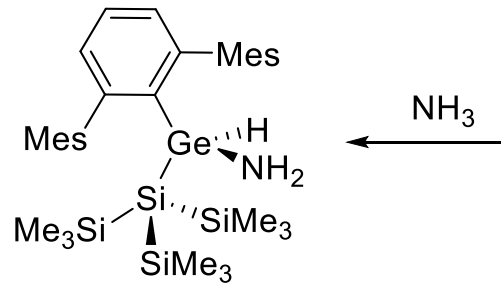

XVI

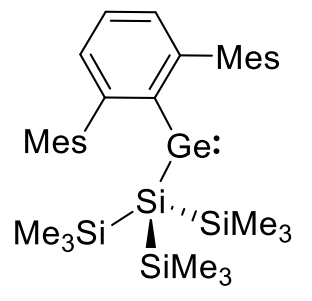

XV

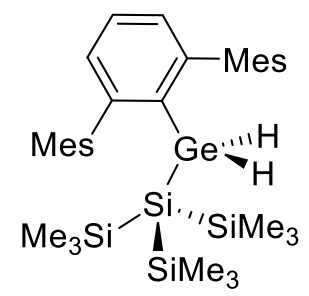

XVII

Scheme 4: Intermolecular $\mathrm{N}-\mathrm{H}$ and $\mathrm{H}-\mathrm{H}$ activation by germylene compound.

Compounds XVI and XVII were confirmed by single crystal X-ray diffraction studies. Widening of the C-GeSi bond angle from $112.47^{\circ}$ to $125.16^{\circ}$ for $\mathrm{XVII}$ and $125.79^{\circ}$ for $\mathrm{XVI}$ is observed. Similar widening of the $\mathrm{X}$ Ge-X angle has also been observed in the formation of other $\mathrm{Si}(\mathrm{IV})$ and $\mathrm{Ge}$ (IV) compounds in oxidative addition reactions of the respective silylene or germylene. ${ }^{8,33}$ This widening due to the reduced size of $\mathrm{Ge}(\mathrm{IV})$ relative to $\mathrm{Ge}(\mathrm{II})$ and $\mathrm{Si}(\mathrm{II})$. The Ge-Si and $\mathrm{Ge}-\mathrm{C}$ bond lengths of $\mathbf{X V I}$ and $\mathbf{X V I I}$ also contract relative to $\mathbf{X V}$ due to the increased electrostatic attraction between the atoms. 
Germylenes with $\mathrm{N}$-heterocyclic ligand systems have also been shown to be reactive towards small molecules. The germylene $\left[\mathrm{CH}\left(\left(\mathrm{C}=\mathrm{CH}_{2}\right)(\mathrm{CMe})\left(2,6-{ }^{i} \mathrm{PrC}_{6} \mathrm{H}_{3} \mathrm{~N}\right)\right)_{2} \mathrm{Ge}\right]$ has been shown to cleave an $\mathrm{N}-\mathrm{H}$ bond of ammonia at room temperature to give XVIII (eq. 10). ${ }^{37}$

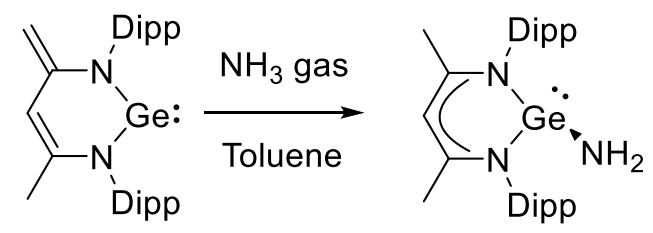

XVIII

Unlike the previous example the reaction does not oxidise the germanium atom, instead the protonation of the terminal methylene results in electron delocalisation in across the ring of the ligand. This results in a lengthening of the Ge-N bonds of the ligand in the solid-state structure from $1.865 \AA$ and $1.866 \AA$ to $2.030 \AA$, also resulting in a narrowing of the $\mathrm{N}-\mathrm{Ge}-\mathrm{N}$ bite angle of the ligand from $95.04^{\circ}$ to $88.60^{\circ}$.

\subsubsection{Imido-Chemistry}

Imido functional groups ( $\left.\mathrm{RN}^{2-}\right)$ are present in both organic and inorganic chemistry. Imido complexes have been shown to exhibit several different binding modes, with each having a unique reactivity. Commonly seen binding modes include the terminal bent binding mode, where the nitrogen atom has a formal double bond to another element (imido if the other element is not carbon, imine if the formal double bond is to a carbon) or symmetrically bridging, where the ligand coordinates to two other elements. Terminal linear imido complexes are extremely short and almost triple bond like in character. They occur primarily with transition metals. ${ }^{38-39}$ In this project germanium imides will be referred to as germanimines to distinguish them from the group 4 compounds, which will be referred to as imido compounds.

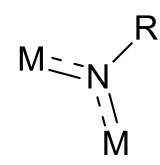

symetrically bridging

$$
\stackrel{\leftarrow}{=} \ddot{N}-R
$$

terminal linear<smiles>[R]N=[N+]=[N-]</smiles>

terminal bent

Figure 4: Common imido coordination modes.

Transition metal imido complexes have been synthesised through a variety of different routes. The first common synthetic method is through the deprotonation of an amido complex. Another well documented 
synthesis is the reaction of halogenated compounds with an amidolithium salt. Alpha abstraction processes have also been used to synthesise imido complexes from diamido metal complexes.

\subsubsection{Germanimine Synthesis and Reactivity}

Germanimine complexes $\left(R_{2} G e=N R^{\prime}\right)$ were first synthesised in the early 1990s from the reaction of sterically protected germylenes with bulky azides. Despite being synthesised over twenty years ago, there are still only 12 examples of germanimines; as such they remain a rarity. ${ }^{40-41}$ The first germanimine XIX, was synthesised by the treatment of the bulky germylene $\left[\mathrm{Ge}\left(\mathrm{N}\left(\mathrm{SiMe}_{3}\right)(\mathrm{Dipp})\right)_{2}\right]$ with $\left[\mathrm{N}_{3}(\mathrm{Dipp})\right]$ in THF at room temperature.

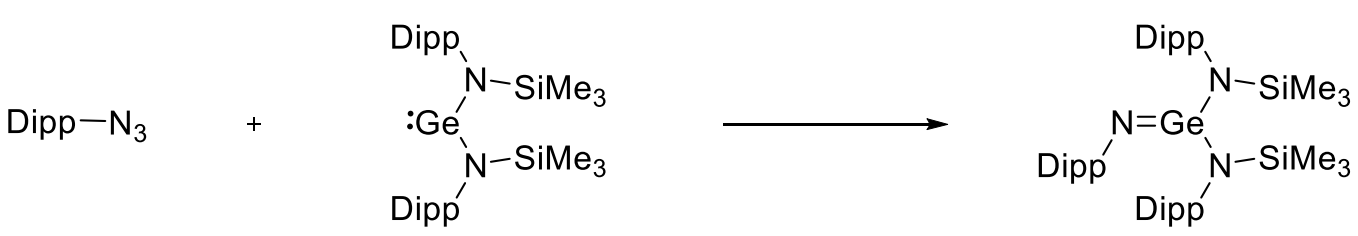

XIX

The single crystal X-ray diffraction study showed the solid state structure had a trigonal planar geometry around the germanium atom $\left(\sum\right.$ internal angles $\left.=359.97^{\circ}\right)$, and a $\mathrm{Ge}=\mathrm{N}$ double bond length of $1.703(2)$

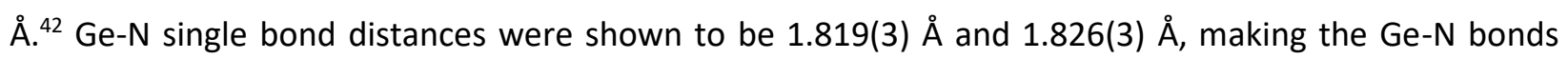
approximately $0.12 \AA$ longer than the $\mathrm{Ge}=\mathrm{N}$ bond. This $0.12 \AA$ contraction was considered a relatively small bond contraction for a double bond, with the authors commenting that most double bond contractions occur more in the order of $0.20 \AA$.

Bis(germanimines) have also been synthesised from the addition of a diazidosilane to bis(bis(trimethylsilyl)methyl)germylene to give the bis(germanimine) $\mathbf{X X}$ with the evolution of a colourless gas (eq. 12). ${ }^{41}$

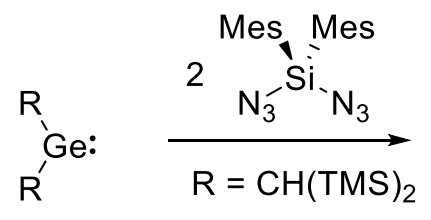

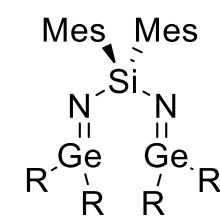

XX 
Wesemann et al. have also synthesised a germanimine XXII using a bulky azide to oxidise the terphenyl stabilised germylene as the kinetic product. ${ }^{43}$ Upon heating the germanimine to $45{ }^{\circ} \mathrm{C}$ for 5 days the germanimine underwent an intramolecular rearrangement to give a phosphinimide $\mathbf{X X I I}$ as the thermodynamic product.

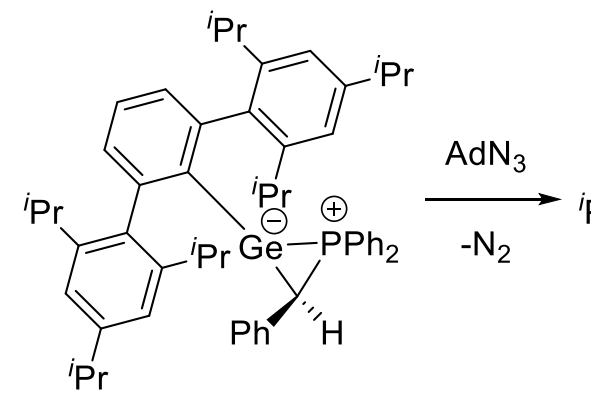

Scheme 5: Synthesis of Wesemann's tetrylene complex using adamantyl azide.

When there is insufficient steric bulk to kinetically stabilise the germanimine, the imine undergoes an [3+2] cycloaddition with an equivalent of azide to form an extremely stable tetrazene product (eq. 13).

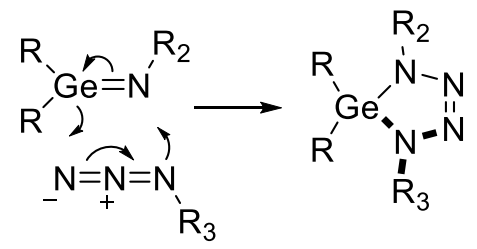

Two such germanes are the $\mathrm{N}$-heterocyclic supported compound XXIII and the bis-amide supported germane XXIV. ${ }^{23,44}$ These reactions highlight the importance of the use of sufficient steric bulk in both the azide and the germylene to sufficiently kinetically stabilise the desired germanimine. 


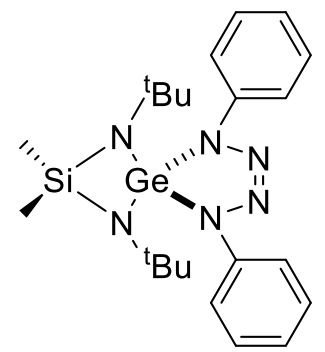

XXIII

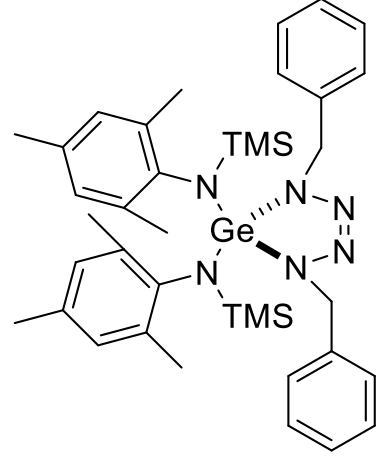

XXIV

Figure 5: Germanes XXIII and XXIV, products of azide insertion into germanimine bond.

\subsubsection{Low oxidation state germanium donor interactions}

Mixed geometry bimetallic germanium amide species have previously been synthesised from reactions of germanium(II) sources with bulky amide ligands. ${ }^{45-46}$ These compounds have been shown to exhibit different geometrical orientations at each germanium atom, and hence have interesting and unique bonding properties that highlight the multiple coordination modes of germanium. Two of these compounds are that of Leung et al.'s germanium-iron heterobimetallic compound $\mathbf{X X V}$ and Jones's bulky germanium dimer $\mathbf{X X V I}$ after activation of hydrogen in the solid state (Figure 6).

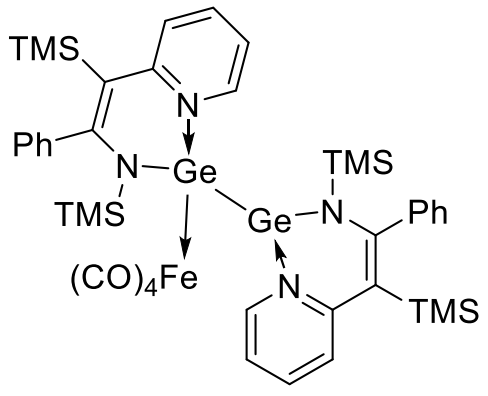

XXV

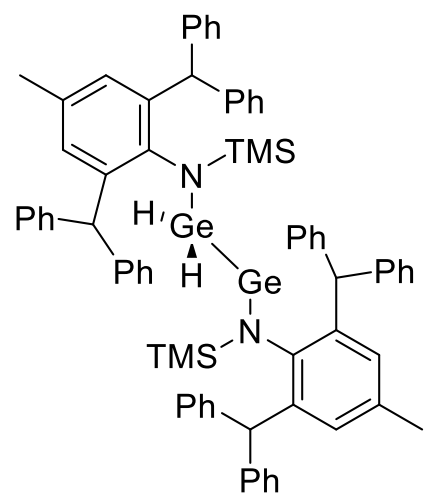

XXVI

Figure 6: Leung's complex (XXV) and Jones's germanium dimer after hydrogen addition (XXVI).

$\mathbf{X X V}$ is a unique heterobimetallic that has germanium atoms present in both a tetrahedral and trigonal pyramidal geometry. The germanium atoms are in the +1 oxidation state, but are both kinetically 
stabilised through the use of steric bulk and electronically stabilised by the donation of nitrogen atom from the pyridyl ring. The lone pair on the one of the germanium atoms acts as a donor to the iron atom of the $\left[\mathrm{Fe}(\mathrm{CO})_{4}\right]$ moiety, while the lone pair on the other germanium atom is acting as a stereochemically active lone pair. In Jones's molecule XXVI, a kinetically stabilised germanium compound, the two germanium atoms are also in different geometries, with the 4-coodinate geometry having a tetrahedral geometry while the 2-coordinate germanium has a bent geometry. The two germanium atoms are in different oxidation states, with the 4-coordinate geometry being in the formal +3 oxidation state while the 2 -coordinate geometry is formally in the +1 oxidation state. These complexes show the high number of oxidation states and coordination numbers available to kinetically stabilised germanium, and highlight its possible potential use in catalytic heterobimetallic complexes.

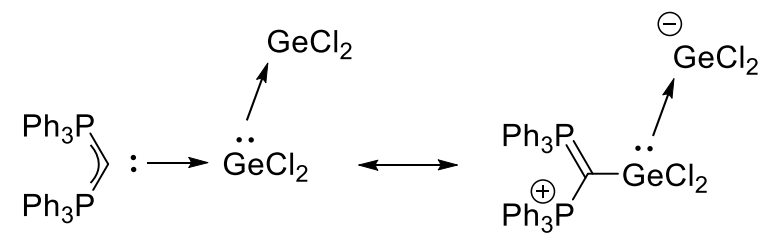

XXVII

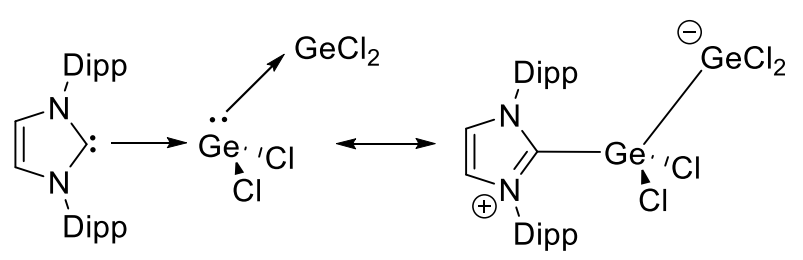

XXVIII

Figure 7: Resonance structures of XXVII and XXVIII.

Alcarazo et al. and Rivard et al. have also described interesting germanium compounds displaying unique donor properties allowing oligerimisation (XXVII and XXVIII). ${ }^{47-48}$ Compound XXVII shows a molecule germanium dichloride supported with a donor carbodiphosphorone ligand. The effect this has on the germanium dichloride molecule is that the lone pair of electrons on the germylene can then donate into an empty $\mathrm{p}$ orbital of another equivalent of germanium dichloride, giving a negative charge on the germanium atom, depending on how the structure is drawn due to its resonance forms (Figure 7). The donation of the lone pair of electrons into the empty $\mathrm{p}$ orbital of the second equivalent of germanium dichloride is highlighted by the almost $90^{\circ}$ bond angles between the to $\mathrm{Ge}-\mathrm{Ge}-\mathrm{Cl}$ bonds. The NHC donor stabilised compound $\mathbf{X X V I I I ~ e x h i b i t s ~ t h e ~ e x a c t ~ s a m e ~ d o n o r - a c c e p t o r ~ i n t e r a c t i o n ~ b e t w e e n ~ g e r m a n i u m ~}$ dichloride units. These compounds show the ability for germanium(II) to be used as a $\sigma$ donor for suitable acceptor compounds. 


\subsection{Group 4 Chemistry}

\subsubsection{Zirconium Imides - Imidozirconocenes}

Donor stabilised imidozirconocene complexes $\left[\mathrm{Cp}_{2} \mathrm{Zr}=\mathrm{NR}(\mathrm{THF})\right]$ could also provide an intermediate towards synthesis of the desired imido bridged zirconium germanium product. The first zirconium bearing a terminal imide $(\mathrm{Zr}=\mathrm{NR})$ was reported in in 1988 as the THF adduct $\left[\mathrm{Cp}_{2} \mathrm{Zr}=\mathrm{N}\left({ }^{t} \mathrm{Bu}\right)(\mathrm{THF})\right] .{ }^{49}$ The compound was formed from $\left[\mathrm{Cp}_{2} \operatorname{Zr}(\mathrm{Me})\left(\mathrm{N}(\mathrm{H})\left({ }^{t} \mathrm{Bu}\right)\right)\right]$, $\mathbf{X X I X}$, which is synthesised either from the reaction of $\left[\mathrm{Cp}_{2} \mathrm{Zr}(\mathrm{Me})_{2}\right]$ with $\left.\left[\mathrm{NH}_{2}{ }^{\mathrm{t}} \mathrm{Bu}\right)\right]$ in benzene at $80^{\circ} \mathrm{C}$, or from the reaction of $\left[\mathrm{Cp}_{2} \mathrm{Zr}(\mathrm{Me})(\mathrm{Cl})\right]$ with the lithium salt [Li][NH $\left.{ }^{t} \mathrm{Bu}\right]$ at $25^{\circ} \mathrm{C}$ in THF. Heating of $\left[\mathrm{Cp}_{2} \mathrm{Zr}(\mathrm{Me})\left(\mathrm{N}(\mathrm{H})\left({ }^{\mathrm{t}} \mathrm{Bu}\right)\right)\right]$ in THF for 3 days at $85^{\circ} \mathrm{C}$ led to the elimination of methane, giving $\left[\mathrm{Cp}_{2} \mathrm{Zr}=\mathrm{N}\left({ }^{t} \mathrm{Bu}\right)(\mathrm{THF})\right], \mathbf{X X X}$ in a $71 \%$ yield (Scheme 6).

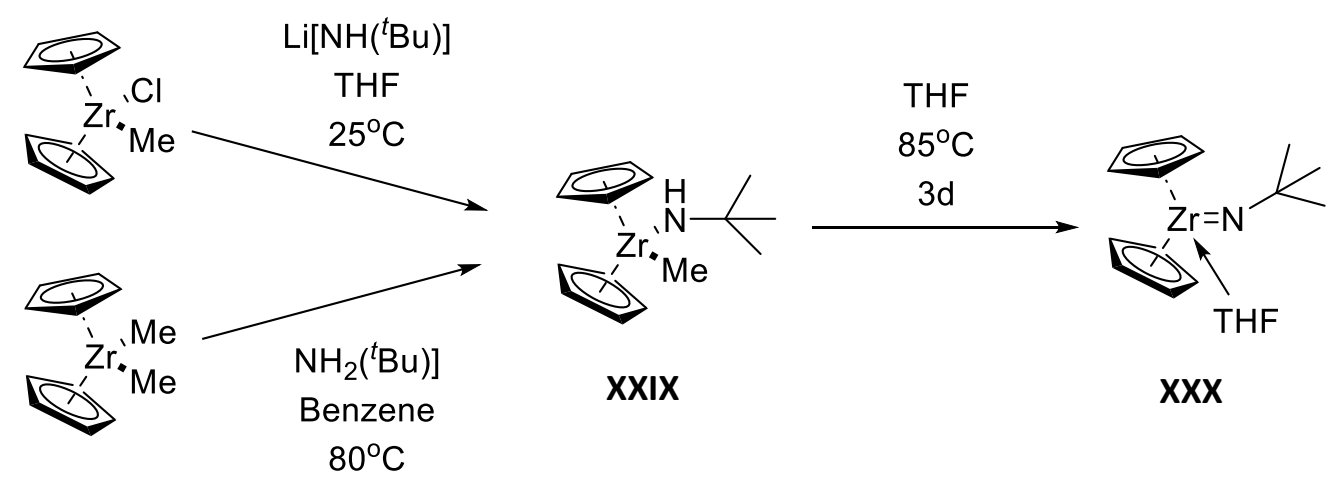

Scheme 6: Synthesis of imidozirconocene XXX through thermolysis to eliminate methane.

More recently, imidozirconocenes have been shown to be synthesised from the readily available starting material $\left[\mathrm{Cp}_{2} \mathrm{ZrCl}_{2}\right]{ }^{50}$ When the lithium amine salt $[\mathrm{Li}][\mathrm{NHR}]\left(\mathrm{R}=4-\mathrm{CMe}_{3}-\mathrm{C}_{6} \mathrm{H}_{4}, 2,6-\mathrm{Me}_{2}-\mathrm{C}_{6} \mathrm{H}_{3}\right)$ is added to $\left[\mathrm{Cp}_{2} \mathrm{ZrCl}_{2}\right]$ in THF at room temperature the alkyl amide $\left[\mathrm{Cp}_{2} \mathrm{Zr}(\mathrm{N}(\mathrm{H})(\mathrm{R}))_{2}\right]$ is formed $\left(\mathrm{R}=4-\mathrm{CMe}_{3}-\mathrm{C}_{6} \mathrm{H}_{4}, 2,6-\right.$ $\left.\mathrm{Me}_{2}-\mathrm{C}_{6} \mathrm{H}_{3}\right)$. Thermolysis of this bisamidozirconocene generated the imidozirconocene through the extrusion of an amine, however; due to the reversible nature of this reaction the imidozirconocene was not isolable. 
The choice of amide is important in the synthesis of the imidozirconocene. Amines without sufficient steric bulk will readily undergo [2+2] cycloadditions upon generation of the imidozirconocene to give the imido bridged dimer (Figure 8). An example of this is the bis( $\mu$-imido) dimer $\left[\mathrm{Cp}_{2} \mathrm{Zr}\left(\mu-\mathrm{N}\left(4-\mathrm{Me}_{3} \mathrm{CC}_{6} \mathrm{H}_{4}\right)_{2}\right)_{2}\right], \mathbf{X X X I}(\mathbf{d})$ which indicates that the para-tert-butyl-phenyl is functionally too small to prevent dimerisation.

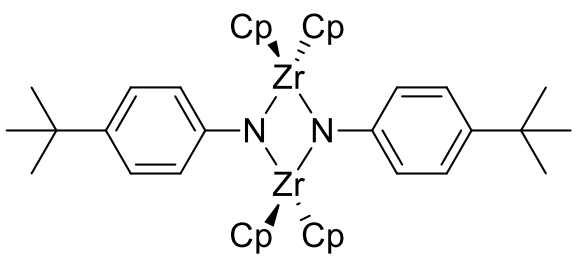

$\operatorname{XXXI(d)}$

Figure 8: Imido bridged zirconocene dimer XXXI(d).

However, tert-butyl and Dipp groups have been shown to be bulky enough to suppress dimerisation of

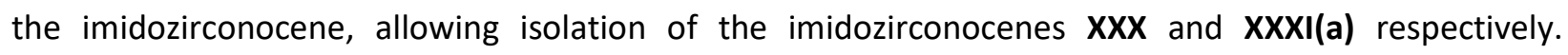
Similarily, when the zirconocene used has one pentamethylcyclopentadienyl ligand (Cp*)instead of a $\mathrm{Cp}$ ligand, the imido complex $\mathbf{X X X I ( b )}$ can also be isolated..$^{51}$ Hence the steric bulk of the $\mathrm{Cp}$ ligands also plays a role on the stability of the imidozirconocene. Figure 9 provides a range of crystallographic data for THF adduct monomer imidozirconocene complexes. ${ }^{52}$

Table 1: Relevant bond distances and angles for a range of imidozirconocene complexes.

\begin{tabular}{|c|c|c|c|}
\hline & $\mathrm{Zr}=\mathrm{N}(\AA ̊)$ & $\mathrm{Zr}-\mathrm{N}-\mathrm{C}\left({ }^{\circ}\right)$ & $\mathrm{Zr}-\mathrm{Cp}(\AA ̊)$ \\
\hline$C p_{2} Z r=N-{ }^{t} B u(T H F), X X X$ & $1.826(4)$ & $174.4(3)^{\circ}$ & 2.325 \\
\hline $\mathrm{C} p_{2} \mathrm{Zr}=\mathrm{N}\left(2,6-{ }^{i} \mathrm{Pr}_{2} \mathrm{C}_{6} \mathrm{H}_{3}\right)(\mathrm{THF}), \mathrm{XXXI}(\mathrm{a})$ & $1.888(2)$ & $159.1(2)^{\circ}$ & $2.312(1), 2.277(1)$ \\
\hline CpCp*Zr=N-p-tol(THF), XXXI(b) & $1.881(3)$ & $168.4(2)^{\circ}$ & $2.2799(3), 2.2725(3)$ \\
\hline $\mathrm{CpCp} * \mathrm{Zr}=\mathrm{N}-{ }^{t} \mathrm{Bu}(\mathrm{THF}), \mathrm{XXXI(c)}$ & $1.846(2)$ & $169.5(2)^{\circ}$ & $2.3268(3), 2.2835(2)$ \\
\hline
\end{tabular}<smiles>[3H][Z]([3H])([3H])=NC(C)(C)C</smiles>

$\mathbf{X X X}$<smiles>[3H][Z]([Te])([Po])=Nc1cccc(C(C)C)c1C(C)C</smiles>

XXXI(a)

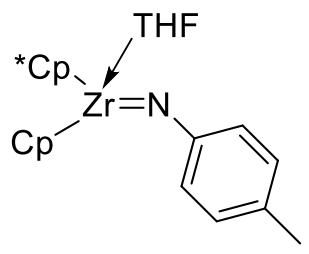

XXXI(b)

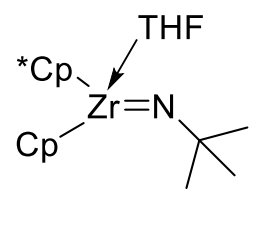

$\mathbf{X X X I ( c )}$

Figure 9: Complexes shown in Table 1. 
The data in Table 1 shows a more obtuse $\mathrm{Zr}-\mathrm{N}-\mathrm{C}$ bond angle in the Dipp substituted imide (XXXI(a)) relative to the other three complexes, reflecting the increased steric bulk at the 2,6 positions of the phenyl ring. Comparing $\mathbf{X X X}$ to $\mathbf{X X X I}(\mathbf{c})$ reveals a decrease in the $\mathrm{Zr}-\mathrm{N}-\mathrm{C}$ bond angle from $174.4(3)^{\circ}$ to $169.5(2)^{\circ}$, indicating that the $\mathrm{Cp}^{*}$ ligand results in a more obtuse bond angle than the $\mathrm{Cp}$ ligand. The $\mathrm{Zr}-\mathrm{N}$ bond length is also longer in $\mathbf{X X X I ( c ) ~} 1.881$ (3) $\AA$ than in $\mathbf{X X X} 1.826(4) \AA$. These structural changes between $\mathbf{X X X}$ and $\mathbf{X X X I}(\mathbf{c})$ are likely a result of the increased electron donating ability and increased steric bulk of the $\mathrm{Cp}^{*}$ ligand. The data in Table 1 also indicates that the alkyl-imido complexes have shorter $\mathrm{Zr}=\mathrm{N}$ distances that the aryl-imido complexes. An example of this is the comparison of $\mathrm{CpCp} * \mathrm{Zr}=\mathrm{N}-p$-tol(THF) and $\mathrm{CpCp} * \mathrm{Zr}=\mathrm{N}$ ${ }^{t} \mathrm{Bu}(\mathrm{THF})$. The increased steric bulk of the tert-butyl over the $p$-toluidine would lead to the assumption that the $\mathrm{Zr}=\mathrm{N}$ distance in $\mathrm{Zr}=\mathrm{N}-{ }^{t} \mathrm{Bu}$ would be longer than in the less sterically hindered $\mathrm{Zn}=\mathrm{N}-p$-tol. However, from the data obtained by the authors this was not the case. Investigating the orbital arrangement of the systems lead to the explanation for the observed lengthening and shortening of the $\mathrm{Zn}=\mathrm{N}$ bond. The $\mathrm{Zr}-\mathrm{N} \sigma$ bond is primarily made up of the interaction between an imide orbital of $\mathrm{a}_{1}$ symmetry and an orbital from the $\mathrm{Cp}_{2} \mathrm{Zr}$ fragment with $2 \mathrm{a}_{1}$ symmetry. The $\pi$ component of the $\mathrm{Zr}-\mathrm{N}$ bond is formed through the interaction between the matching $b_{2}$ orbital of the imide and zirconium respectively. The third nitrogen based orbital of $b_{1}$ symmetry cannot interact with the vacant $a_{1}$ orbital on the zirconium fragment, as the only orbital on the zirconium fragment with $b_{1}$ symmetry is a high energy orbital Zr-Cp antibonding character. The poor energetic overlap between the imide and zirconium based $\mathrm{b}_{1}$ orbitals is probably a weak interaction due to this significant energy difference. As such the $\mathrm{Zr}-\mathrm{N}$ interaction can best be thought of as a double bond with a large, filled nonbonding orbital present on the nitrogen atom. ${ }^{53}$ However, the disparity in bond lengths between the aryl and alkyl substituted imidozirconocenes indicate that the imide $b_{1}$ symmetry orbital is probably not entirely dissociated from the $\mathrm{Zr}-\mathrm{N}$ bonding. The lengthening of the $\mathrm{Zr}-\mathrm{N}$ bond in the arylimido species indicates that the nitrogen based $b_{1}$ orbital of the $N$-arylimido complex is stabilized by the $\pi$ system of the $N$-aryl substituent. This separates the $\mathrm{Zr}$ fragment and imide based $\mathrm{b}_{1}$ orbitals further, reducing the impact on the $\mathrm{Zr}-\mathrm{N}$ bonding. This stabilisation is not present in the alkylimide substituents, and as such, the energy matching between the $b_{1}$ orbitals of the nitrogen and zirconium fragment is slightly improved, resulting in a stronger interaction (a shorter bond). 
As the molecular orbital arising from the interaction of the two aforementioned $b_{1}$ orbitals has antibonding character, the $\mathrm{Zr}$-Cp distance observed would be expected to decrease for the alkyl imidozirconocene complexes over the aryl imidozirconocene complexes. This is observed in the data in Table 1, in which there is a 0.05-0.06 ̊̊ increase in the Zr-Cp bond lengths.

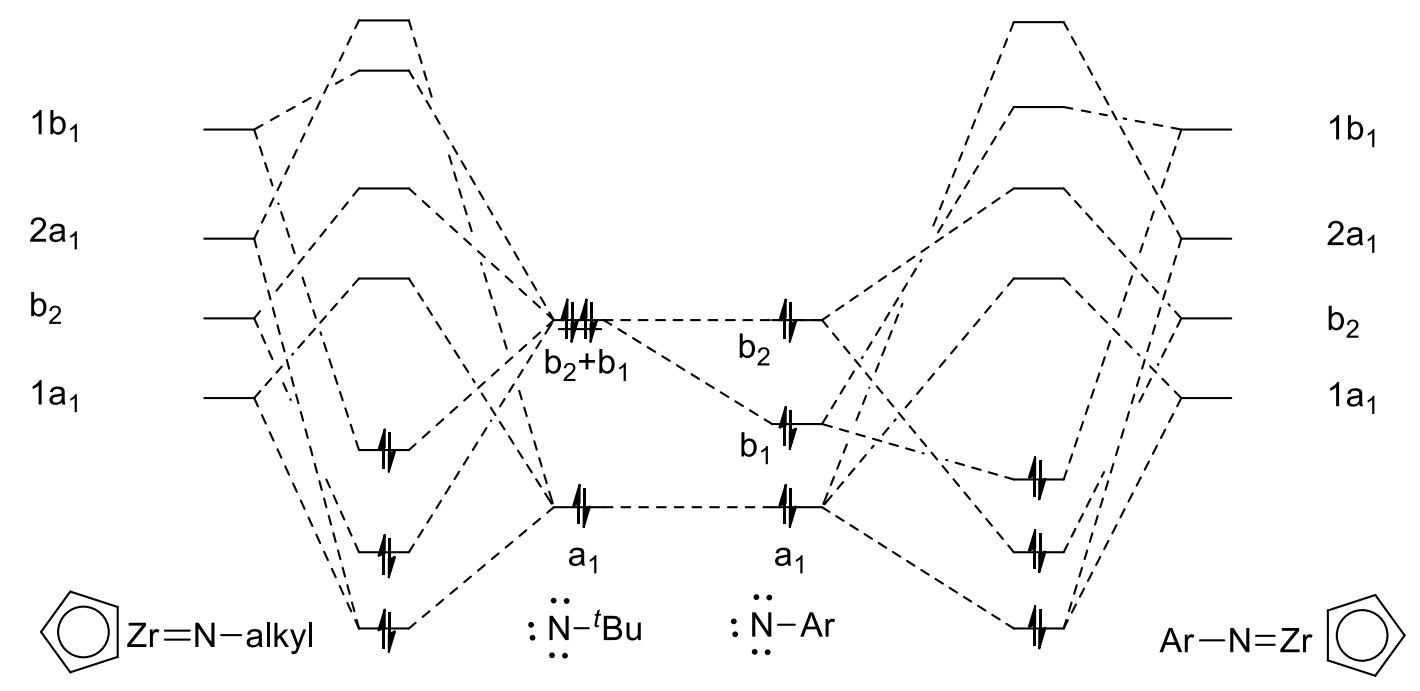

Figure 10: Relative orbital energy diagram of alkyl and aryl imidozirconocenes.

Imidozirconocenes have also been shown to undergo a range of reactions with various substrates. One such reaction is the $[2+2]$ cycloaddition between imidozirconocenes and small unsaturated molecules such as heterocumulenes. ${ }^{54}$ When the THF adducts of the imidozirconocenes $\mathrm{Cp}_{2} \mathrm{Zr}=\mathrm{N}(\mathrm{R})$ were treated with various symmetrical carbodiimides, diazametallacycles were formed in high yields (Scheme 7).

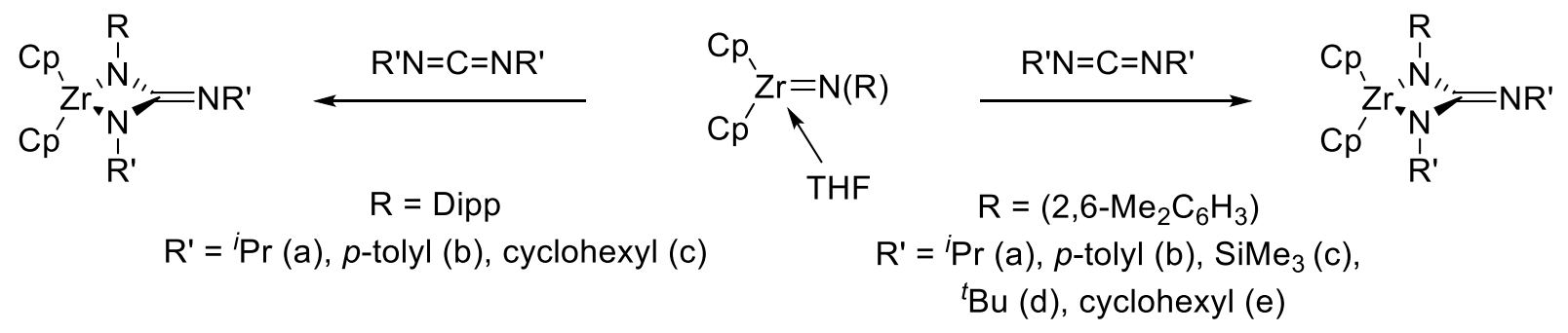

XXXII(a), (b) and (c)

XXXIII(a), (b), (c), (d) and (e)

Scheme 7: Reactions of imidozirconocenes with various heterocumulenes. 
Increasing the steric bulk of the imidozirconocene resulted the [2+2] cycloaddition reaction occurring only in the case of less bulky carbodiimides such as ${ }^{i} \mathrm{PrN}=\mathrm{C}=\mathrm{N}^{i} \mathrm{Pr}$. Although there were two possible regioisomers as potential products of the cycloaddition, one in which a zirconium carbon bond would be formed and one in which a zirconium nitrogen bond would be formed, only one of the products (the zirconium nitrogen bond) was formed.

As imidozirconocenes readily undergo [2+2] cycloadditions they have also been investigated for their potential use as catalysts in imine metathesis and in the hydroamination of alkynes. ${ }^{51}$ When $\mathrm{CpCp} * \mathrm{Zr}=\mathrm{N}^{t} \mathrm{Bu}(\mathrm{THF})$ is used at a catalyst for the olefin metathesis of $p-\mathrm{TolCH}=\mathrm{CH}-p-\mathrm{Tol}$ with $\mathrm{PhCH}=\mathrm{CHPh}$ a 1:1:1:1 equilibrium mixture of the imines $p$-TolCH=NPh amd $\mathrm{PhCh}=\mathrm{N}-p$-Tol were generated along with the alkene starting materials. (Scheme 8).

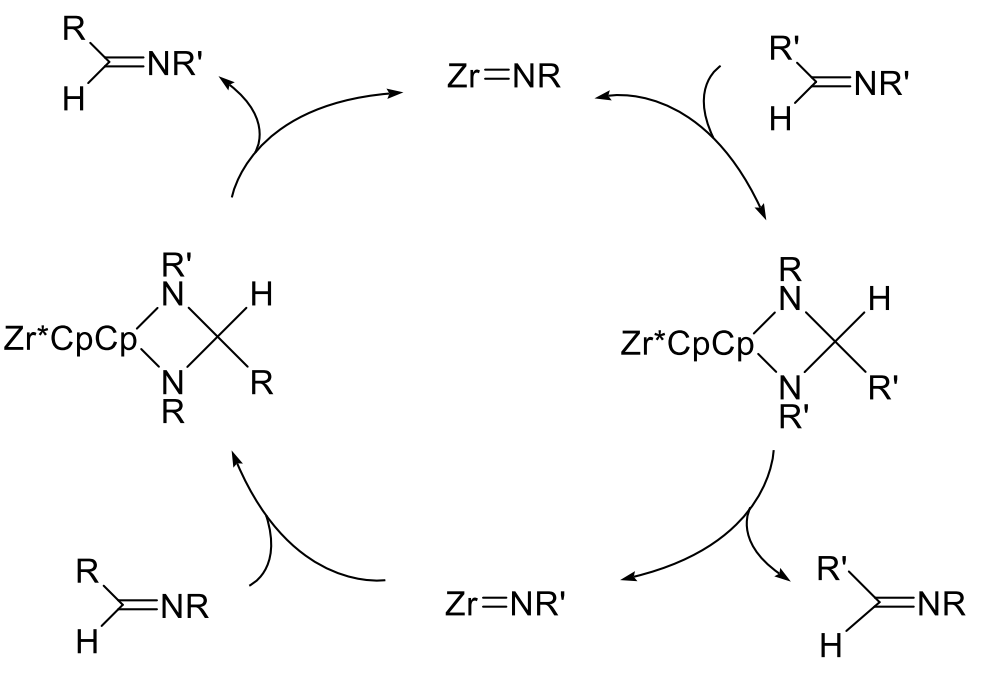

Scheme 8: Catalytic cycle of imidozirconocenes mediated imide metathesis.

The use of imidozirconocenes as catalysts for the hydroamination of alkynes has also been achieved (Scheme 9). 


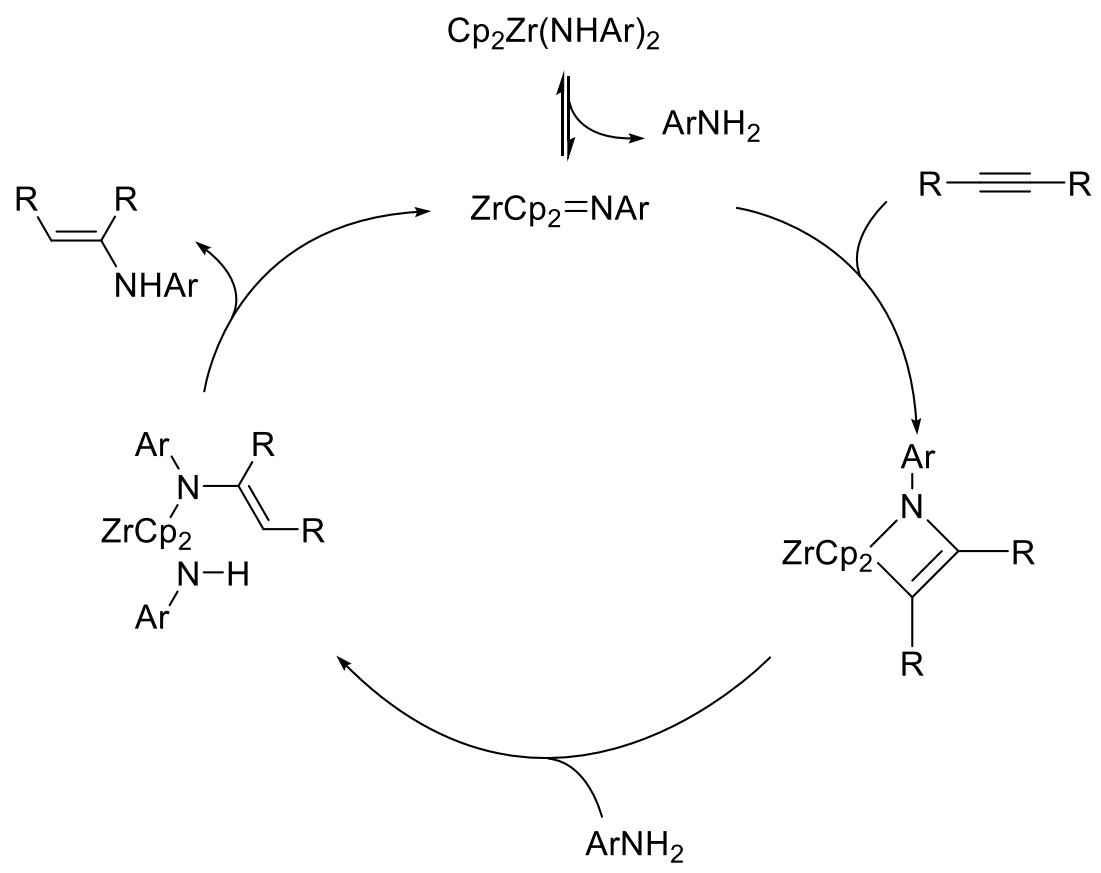

Scheme 9: Catalytic cycle of imidozirconocene mediated alkyne hydroamination.

Imidozirconocenes have also had their reactivity towards azides investigated. ${ }^{55-57}$ The imidozirconocene have been shown to undergo [3+2] cycloadditions with azides to form tetrazene products (Scheme 10). Compound $\operatorname{XXXIV(a)~was~characterised~by~a~single~crystal~X-ray~diffraction~study.~The~Zr-N~bond~lengths~}$ were $1.380 \AA$, longer than the the $\mathrm{N}=\mathrm{N}$ bond length of $1.281 \AA$. The tetrazene ligand had a $\mathrm{N}-\mathrm{Zr}-\mathrm{N}$ bond angle of $75.2^{\circ}$, smaller to that of XXIII $\left(82.62^{\circ}\right)$ and XXIV $\left(82.21^{\circ}\right)$.

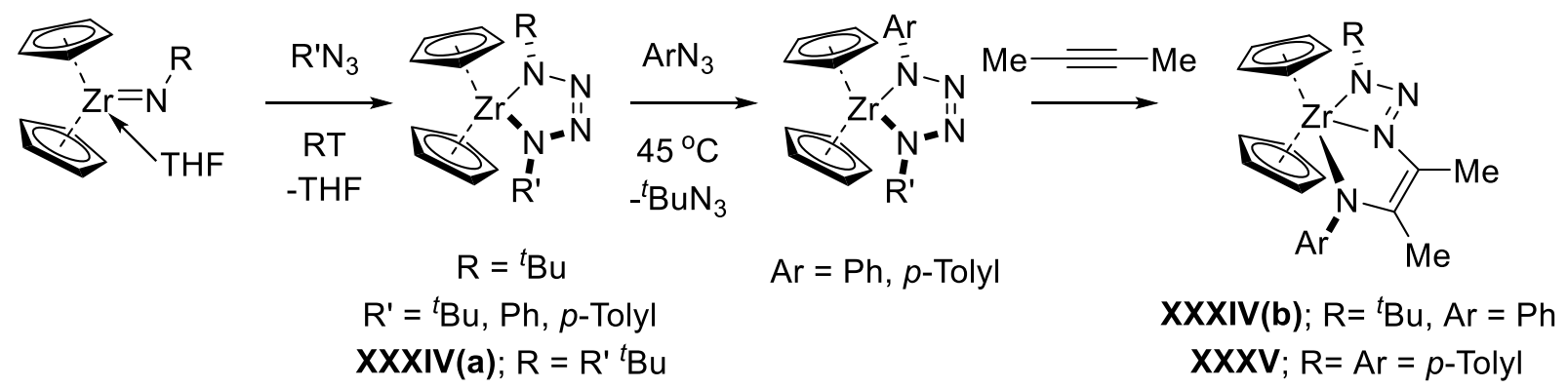

Scheme 10: Synthesis and reactivity of tetrazene zirconium complexes from an imidozirconocene. 
These tetrazene compounds have also been shown to react with methylacetylene, with the methylacetylene inserting into the $\mathrm{N}-\mathrm{N}$ bond to synthesise the insertion products $\mathbf{X X X I V ( b )}$ and $\mathbf{X X X V}$. Of these products, only $\mathbf{X X X V}$ has been characterised by $\mathbf{X}$-ray diffraction, and provides an interesting comparison to the triazenido ligand due to the lack of resonance structures and structural rigidity provided by the $\mathrm{NC}=\mathrm{CN}=\mathrm{N}$ bonding motif. $^{58}$

\subsubsection{Zirconium-Germanium compounds}

Zirconium germanium compounds are rare, but there have been two examples of a $\mathrm{Zr}$-Ge bond being characterised by X-ray diffraction. The transmetallation reaction of 1,2dipotassiotetrakis(trimethylsilyl)digermane with magnesium bromide followed treatment with zirconocene dichloride results in the zirconocenedigermane complex XXXVI (Scheme 11). ${ }^{59}$ The only characterisation of this compound was by ${ }^{1} \mathrm{H}$ NMR spectroscopy. When the compound $\mathbf{X X X V I}$ was treated with trimethylphosphine, the phosphine adduct XXXVII was isolated in the solid state and characterised by an X-ray diffraction study. The ${ }^{29} \mathrm{Si}$ NMR spectrum suggested partial germylene character of the coordinated digermane.

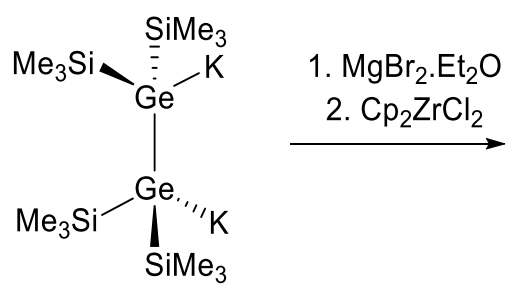

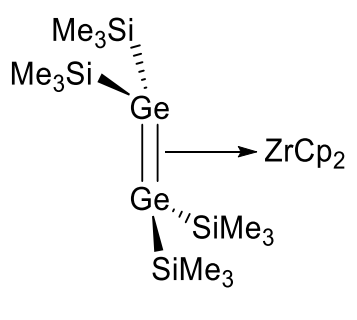

XXXVI

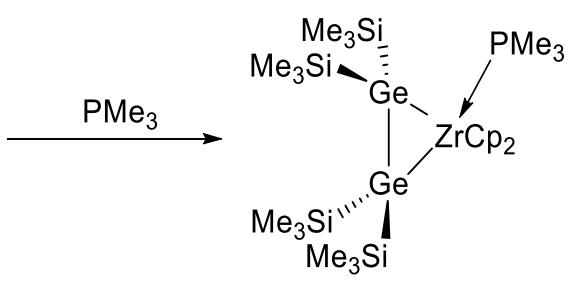

XXXVII

Scheme 11: Synthesis of XXXVII from XXXI.

More recently the reaction of a disilylated five membered cyclic germylene with a range of group IV metallocene dichlorides has been reported. ${ }^{60}$ The reduction of the group IV metallocene dichlorides with 
magnesium in the presence of the germylene triethylphosphine adduct in THF gave the metallocene germylene compounds as triethylphosphine adducts (eq. 14).
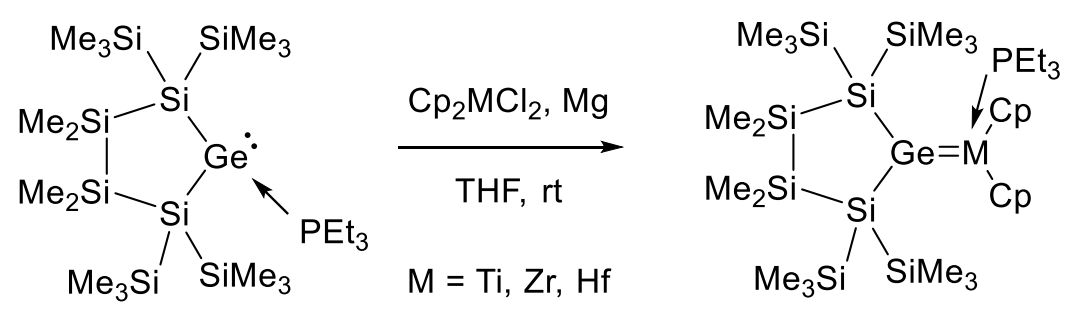

\section{XXXVIII}

The products were isolated and characterised using single crystal X-ray diffraction. For all three products the germanium atom sits in the metallocenes equatorial plane, with the bidentate silicon chelating ligand near orthogonal to this plane. This is also observed in the equivalent stannylene and plumbylene compounds, as well as known titanocene and hafnocene silylene complexes isolated as either trimethylphosphine, THF or isonitrile aducts. ${ }^{61-63}$ The authors propose that this orientation leads to favourable orbital overlap between transition metals filled $d$ orbitals and the empty germylene $p_{\pi}$ orbital. For complex XXXVIII, the Ge-Zr bond distance was found to be $2.63 \AA$ in the solid state, significantly shorter than the $2.87 \AA$ and $2.89 \AA$ Ge-Zr bond previously reported for the aforementioned zirconocene germane species. This shortening of the bond reflects the double bond character associated with the heterobimetallic complex XXXVIII. The bonding in the complex is described by a $\sigma$-bonding, $\pi$ backbonding Scheme shown in Figure $11 .{ }^{60}$ 

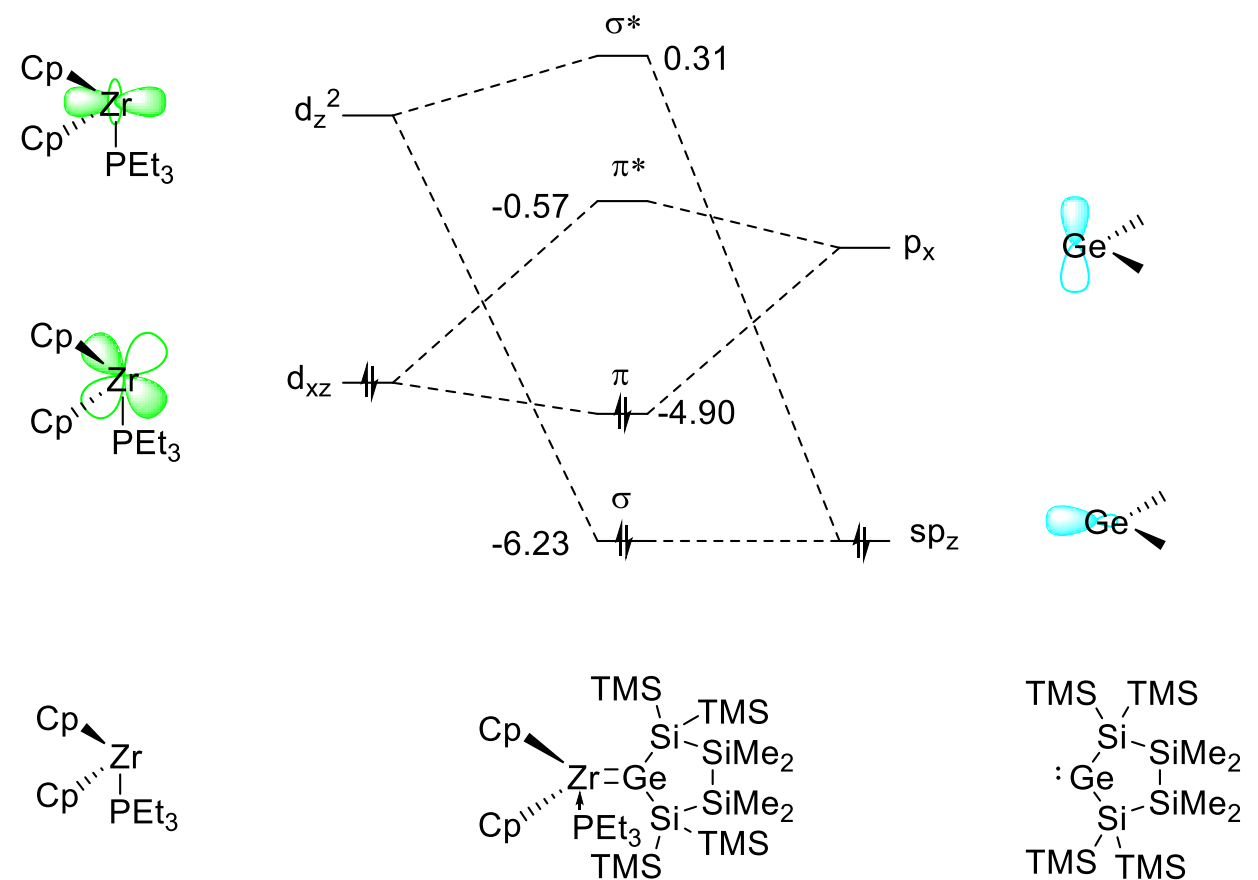

TMS TMS

Figure 11: Orbital energy diagram showing bonding interaction of the heterobimetallic complex XXVII and relative stabilisation and destabilisation of the $\pi$ and $\pi^{*}$ orbitals.

\subsection{Imido-Bridged Bimetallic Compounds Synthesis and Reactivity}

Early-late transition metal heterobimetallics have been studied in the past due to the complementary reactivity of the two different transition metals present in the compounds. The electron-rich late transition metal (often $\mathrm{Rh}, \mathrm{Ir}, \mathrm{Fe}$, or $\mathrm{Mo}$ ), and electron-poor early transition metal create an ideal environment for heterolytic bond cleavage in what is often termed 'cooperative reactivity'. ${ }^{64-65}$

A heterobimetallic compound is any compound that contains in its structure two different metal atoms. These compounds often have unique chemistry that is significantly different from the properties of the individual metals, are hence are of interest due to the unique and unpredictable properties these compounds contain. 
The synthesis of the early-late imido bridged heterobimetallic $\left[\mathrm{Cp}_{2} \mathrm{Zr}\left(\mu-\mathrm{N}-{ }^{t} \mathrm{Bu}\right)-\mathrm{IrCp}{ }^{*}\right]$ has previously been reported..$^{65}$ The addition of 2 equivalents of $n$-BuLi to $\left[\mathrm{Cp}_{2} \mathrm{ZrCl}_{2}\right]$ at $-30{ }^{\circ} \mathrm{C}$ in THF followed by addition of $\left[\mathrm{Cp}^{*} \mathrm{Ir} \equiv \mathrm{N}-{ }^{\mathrm{t}} \mathrm{Bu}\right]$ led to compound $\mathbf{X X X I X}$ (Scheme 12).

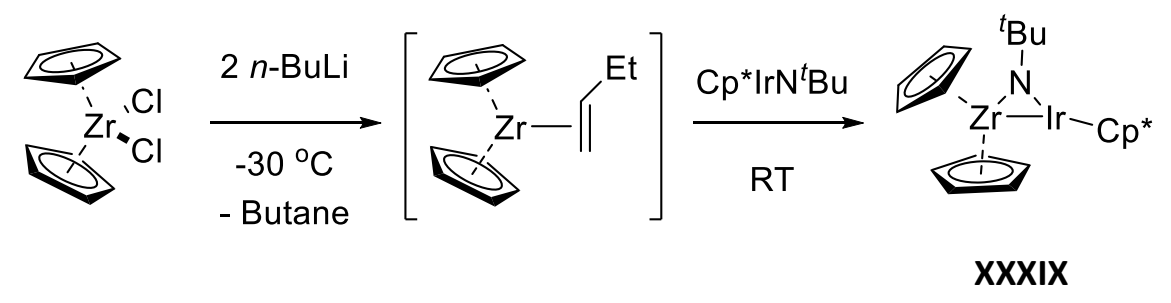

Scheme 12: Synthesis of heterobimetallic XXXIX via the Negishi reagent.

Compound XXXIX was subjected to a wide reactivity study with $\mathrm{H}_{2}, \mathrm{PhMeSiH}_{2}, \mathrm{H}_{2} \mathrm{NAr},{ }^{t} \mathrm{BuOH}, p$-tolylOH, $\left(\mathrm{CH}_{3}\right)_{2} \mathrm{CO}, \mathrm{H}_{2} \mathrm{~S}$ and $\mathrm{HPEt}_{2}$. The resulting compounds from addition of these compounds is shown in Scheme 13. Compound XXXIX reacts with both polar and non-polar substrates. Most reactions occur as addition reactions across the Ir-Zr bond, as these centers are both electron deficient regarding the 18 electron rule (both $\mathrm{Zr}$ and $\mathrm{Ir}$ have 16 electron configurations). In this reactivity, zirconium acts as a Lewis acid while iridium acts as a Lewis base.

The complex XXXIX was shown to react with X-H bonds $\left(X=H, S i P h(H) M e, N H A r, O-p\right.$-tolyl, O- ${ }^{t} B u$, $\mathrm{CH}_{2} \mathrm{C}(\mathrm{O}) \mathrm{CH}_{3}$ ) to form a new metallacycle with a bridging hydride ligand coordinated to the iridium and zirconium centres, and the $X$ functionality bound to the zirconium centre (Scheme 13). The proposed mechanism for these reactions involves the complexation of the hard heteroatom to the zirconium center, followed by a proton transfer to the iridium center. 


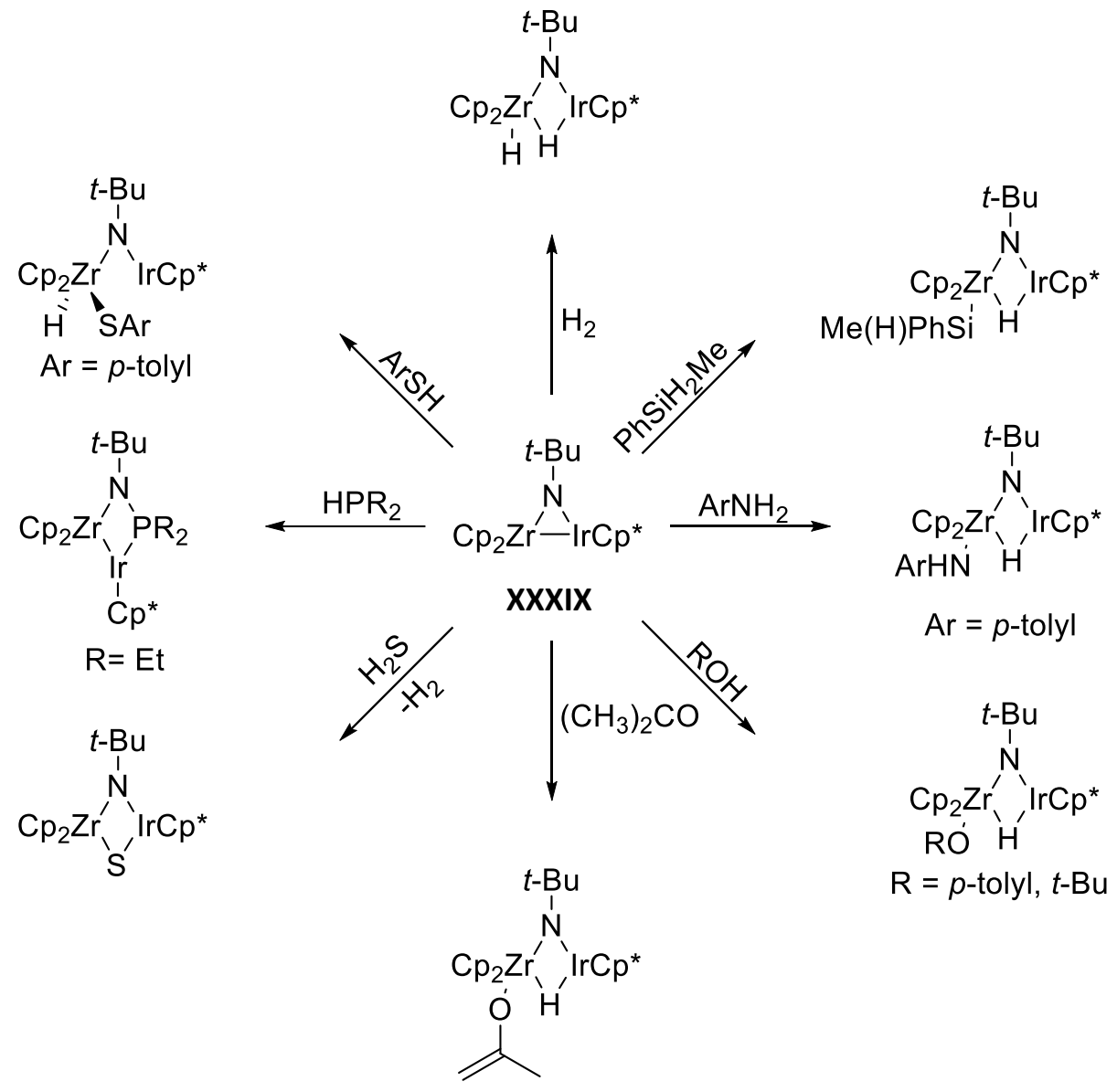

Scheme 13: Reactivity of complex XXXIX with a range of polar small molecules.

However, complex XXXIX also showed different reactivity towards other substrates. The reaction with $\mathrm{H}_{2} \mathrm{~S}$ produced a new metallacycle with a bridging sulfur ligand and the evolution of hydrogen gas. The addition of the more sterically demanding $p$-thiocresol resulted in two products in equilibrium; the product shown in Scheme 14, as well as the hydrogen bridged complex with the thiol on the zirconium atom. The reaction of complex XXXIX with the phosphine HPEt ${ }_{2}$ also produced a new metallacycle, with a bridging phosphine. However, the insertion of the phosphine into the complex was not between the Ir-Zr bond, but the Ir-N bond.

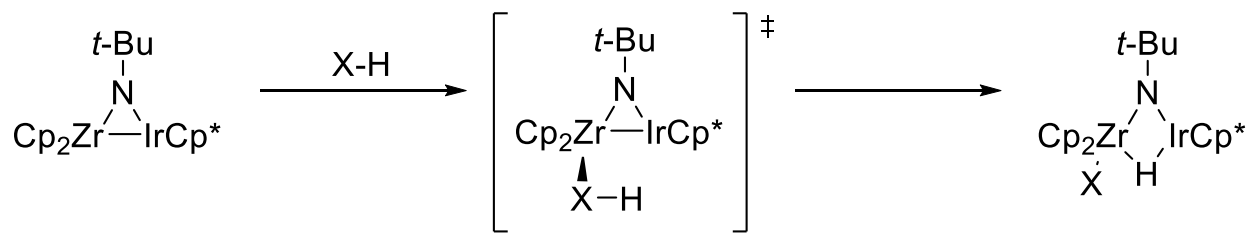

Scheme 14: X-H Addition to complex XXXIX. 


\section{Results and Discussion: Group 14 Chemistry}

This chapter discusses the synthesis and reactivity of some novel germanium compounds including germylenes, mixed coordination germanium compounds and germanimines. The germylenes and germanimines were synthesised for their use in the synthesis of $\mathbf{1}$, while the mixed coordination compounds are discussed due to their unique bonding interactions. Density functional theory (DFT) molecular orbital calculations were also used to investigate the reactivity and bonding in the germylenes and mixed coordination germanium compounds. Finally, DFT was also used to investigate the change in structure and bonding of the heavier group 14 complexes $(\mathrm{M}=\mathrm{Ge}, \mathrm{Sn}, \mathrm{Pb})$ for the $[(1 \mathrm{E})-N 1, N 2-$ dimesitylacenaphthylene-1,2-diimine $] \quad\left[\mathrm{BIAN}^{\mathrm{Mes}}\right] \quad$ and $\quad[N 1, N 8$-bis(trimethylsilyl)naphthalene-1,8diamine]; [NAPH ${ }^{\mathrm{TMS}}$ ] ligands.

\subsection{Germylene Synthesis}

$\mathrm{N}$-heterocyclic germylenes were synthesised due to their ease of synthesis and the ready availability of the required starting materials. As previously mentioned, the properties of NHGes over other germylene varieties is limited, however due to the advantages discussed earlier a mix of known and previously unknown NHGe species were synthesised.

\subsection{1 [Ge(BIAN $\left.\left.{ }^{M e s}\right)\right]$ synthesis 2}

[Ge(BIAN $\left.\left.{ }^{M e s}\right)\right], 2$ was synthesised in a two-step process. Addition of [(1E)-N1,N2-dimesitylacenaphthylene1,2-diimine]; [BIAN ${ }^{\mathrm{Mes}}-\mathrm{H}$ ] to magnesium turnings in THF resulted in the formation of a green solution after 3 days at $100{ }^{\circ} \mathrm{C}$, from which dark violet [(BIAN $\left.\left.{ }^{\mathrm{Mes}}\right) \mathrm{Mg}\left(\mathrm{THF}_{3}\right)\right]$ was isolated. Compound $\mathbf{2}$ was then formed by addition of a THF solution of this compound to $\mathrm{GeCl}_{2}$.dioxane at $-30{ }^{\circ} \mathrm{C}$. 


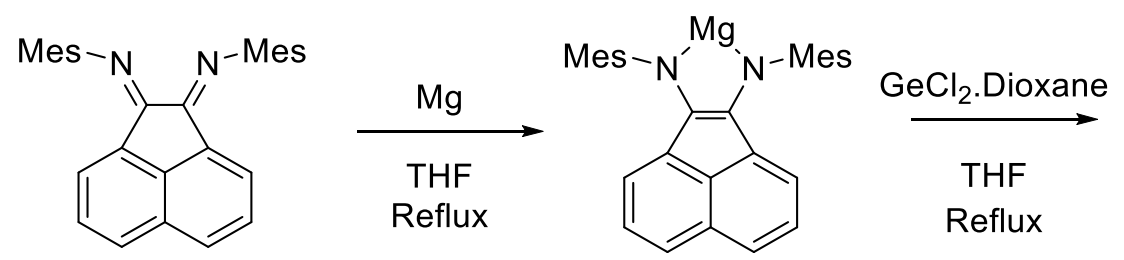

Scheme 15: Synthesis of 2 from [BIAN ${ }^{\text {Mes }}$ preligand.

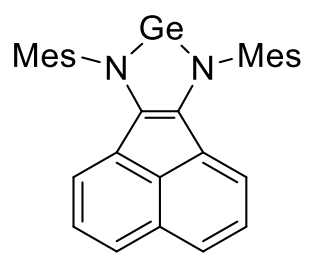

2

The ${ }^{1} \mathrm{H}$ NMR spectrum of 2 revealed two singlets at $\delta 2.29$ and $2.26 \mathrm{ppm}$ integrating to 12 and 6 protons respectivley, corresponding to the methyl groups on the $N$-mesityl functional group. The resonance at $\delta$ $6.92 \mathrm{ppm}$ integrating for $4 \mathrm{H}$ is assigned to the meta-protons of the $\mathrm{N}$-mesityl group. Three additional resonances were found corresponding to the ortho, meta and para position of the naphthalene backbone at $\delta 6.59$ (doublet), 6.90 (triplet), and 7.20 (doublet), respectively. The ${ }^{1} \mathrm{H}$ NMR spectrum is indicative of a complex with a plane of symmetry.

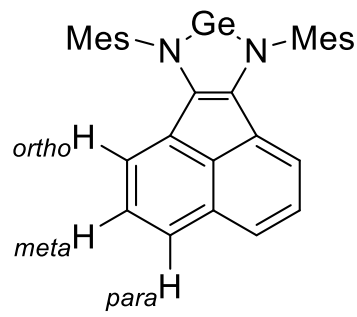

2

Figure 12: Ortho, meta and para positions of 2.

Dark brown crystals suitable for an X-ray diffraction study were grown after layering a concentrated toluene solution of $\mathbf{2}$ with hexane and letting stand at room temperature for 5 days. The solid-state crystal structure of [ $\left.\mathrm{Ge}\left(\mathrm{BIAN}^{\mathrm{Mes}}\right)\right]$ reveals two molecules in the asymmetric unit (molecule $\mathrm{A}$ and $\mathrm{B}$ ) with similar bond lengths and angles, as such, only one molecule A will be described. The angle between the mesityl group and BIAN backbone in molecule $A$ is $86.19^{\circ}$ and $87.61^{\circ}$. This angle is similar to that of other BIANgermanium complexes. For instance, in compound IV(a) the angle between the $\mathrm{N}$-aryl plane and the BIAN backbone is $86.15^{\circ}$ and $88.98^{\circ}$. The planes of the phenyl rings in each of the mesityl groups do not sit in plane with each other and instead have an angle between planes of $8.36^{\circ}$. The Ge-N bond length of molecule $A$ is 1.891 and $1.880 \AA$, similar to IV(a) (1.885 and $1.897 \AA$ ) but shorter than IV(c) (1.904 and $1.915 \AA$ ) and longer than IV(b) (1.878 $)$. These are also longer than the Ge-N bond lengths of $\mathbf{V}$ of 1.864 $\AA$. The $N(3)-G e(2)-N(4)$ bite angle of $84.39^{\circ}$ is similar to $\operatorname{IV}(a), \operatorname{IV}(b)$ and $\operatorname{IV}(c)\left(85.20^{\circ}, 85.03^{\circ}\right.$ and $\left.84.01^{\circ}\right)$, but larger than $\mathbf{V}\left(82.84^{\circ}\right)$, indicating that the different backbone of $\mathbf{2}$ has the germanium atom sitting further in the binding pocket of the ligand. ${ }^{25}$ 


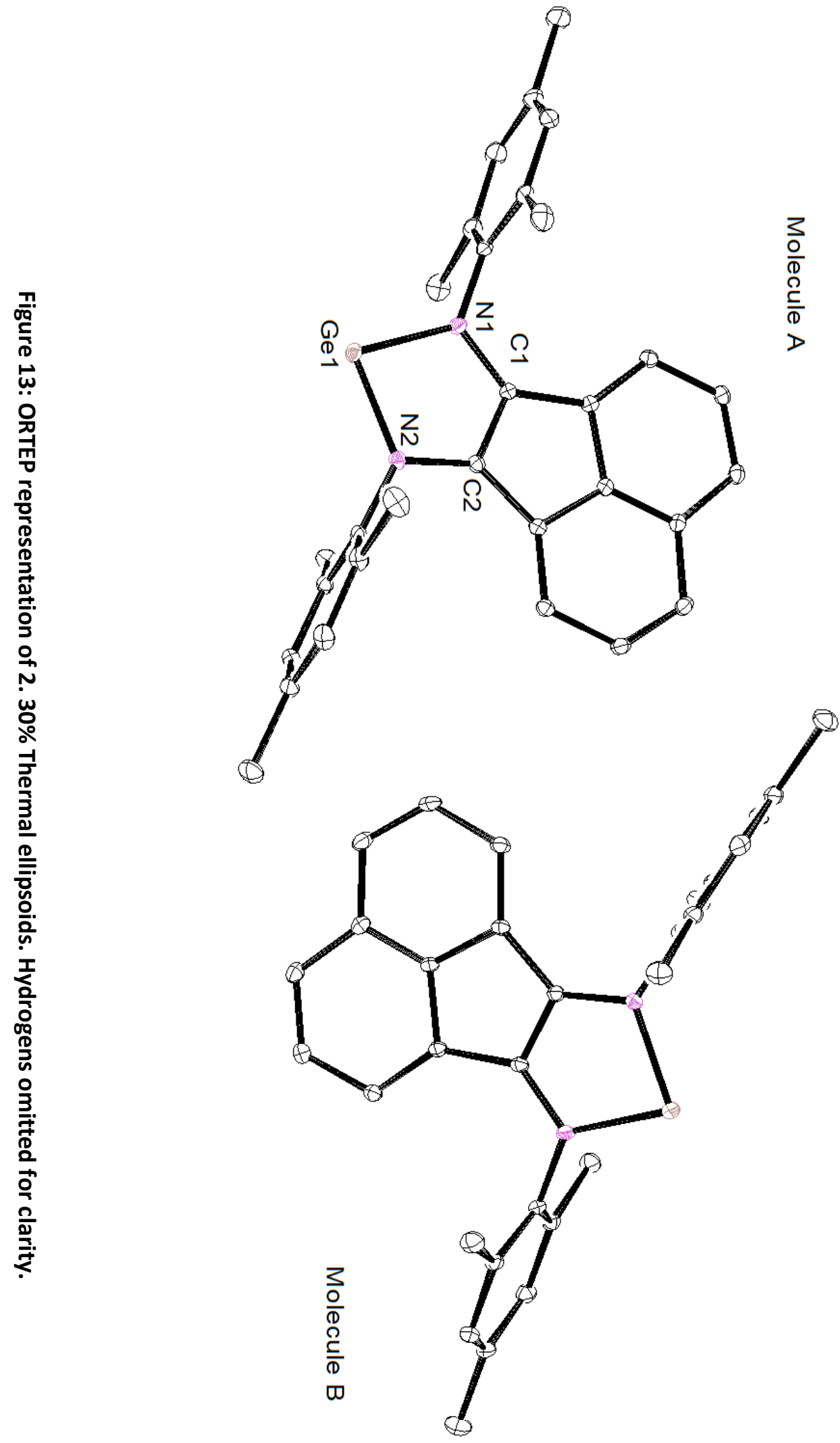


Table 2: Selected bond lengths and angles of 12.

\section{Bond Lengths ( $\AA$ )}

$\begin{array}{ll}\mathrm{Ge}(1)-\mathrm{N}(1) & 1.894(2) \\ \mathrm{Ge}(1)-\mathrm{N}(2) & 1.893(2) \\ \mathrm{N}(1)-\mathrm{C}(13) & 1.369(3) \\ \mathrm{N}(2)-\mathrm{C}(22) & 1.370(3) \\ \mathrm{C}(1)-\mathrm{C}-(2) & 1.379(4) \\ \mathrm{N}(1)-\mathrm{C}(1) & 1.435(3) \\ \mathrm{N}(2)-\mathrm{C}(2) & 1.429(3)\end{array}$

\section{Bond Angles ( $\left.{ }^{\circ}\right)$}

$\begin{array}{cc}N(1)-G e(1)-N(2) & 84.39(9) \\ G e(1)-N(1)-C(13) & 129.08(16) \\ G e(1)-N(2)-C(22) & 127.31(15) \\ N(1)-C(1)-C(3) & 135.2(2) \\ N(1)-C(1)-C(2) & 115.0(2) \\ N(2)-C(2)-C(11) & 135.3(2) \\ N(2)-C(2)-C(1) & 115.3(4)\end{array}$

\subsection{2 $\left[\mathrm{Ge}\left(\mathrm{NAPH}^{\mathrm{TMS}}\right)\right]$ synthesis 3}

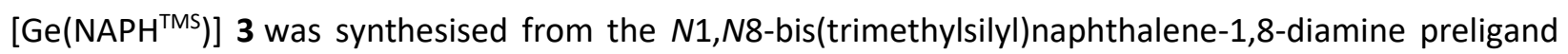
[NAPH ${ }^{\mathrm{TMS}}-\mathrm{H}$ ] via lithiation and salt methathesis. Lithiation with 2 equivalents of $n$-BuLi afforded the lithiated intermediate salt of the ligand which underwent salt metathesis with $\mathrm{GeCl}_{2}$.dioxane in THF to give the germylene [Ge(NAPH $\left.\left.{ }^{\mathrm{TMS}}\right]\right)$ as orange crystals. [Ge( $\left.\mathrm{NAPH}^{\mathrm{TMS}}\right)$ ] was characterized by ${ }^{1} \mathrm{H} \mathrm{NMR}$ spectroscopy, ${ }^{13} \mathrm{C}$ NMR spectroscopy, UV-Vis spectroscopy and single crystal X-ray diffraction.

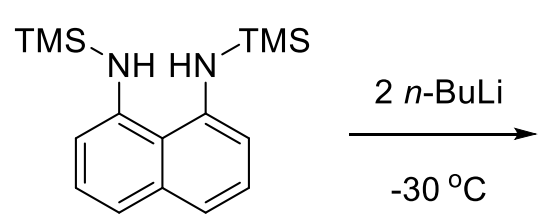

Scheme 16: Synthesis of 3 using [NAPH ${ }^{\mathrm{TMS}}$ ] ligand.

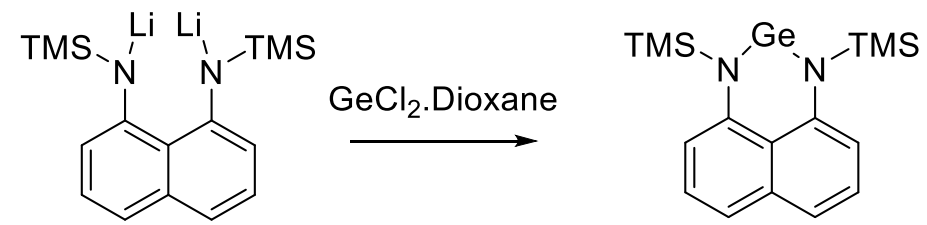

3

The ${ }^{1} \mathrm{H}$ NMR spectrum of $\mathbf{3}$ has a downfield shift in resonance attributed to the $\mathrm{N}$-TMS functional group from [NAPH ${ }^{\mathrm{TMS}}-\mathrm{H}$ ] to $\delta 0.34 \mathrm{ppm}$ from $0.18 \mathrm{ppm}$. The three aromatic peaks in the naphthalene backbone are seen as a doublet of doublets at $\delta 7.21 \mathrm{ppm}$ for the para protons, a triplet at $\delta 7.13 \mathrm{ppm}$ for the meta protons and a doublet of doublets at $\delta 6.71 \mathrm{ppm}$ for the ortho protons. 


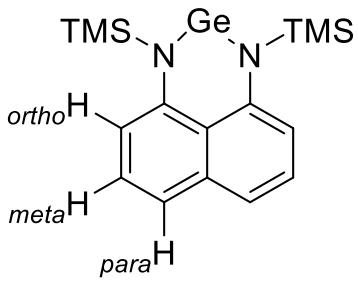

3

Figure 14: Ortho, meta and para positions of 3.

Crystals suitable for an X-ray diffraction study were grown from a concentrated solution of $\mathbf{3}$ in hexane. The solid-state crystal structure data of $\mathbf{3}$ shows one molecule in the asymmetric unit. The molecule shows a twisted naphthalene backbone with one of each of the nitrogen atoms sitting above or below the plane of the naphthalene ring. The $N$-TMS groups also sit above $\mathrm{Si}(2)$ and below $\mathrm{Si}(1)$ the naphthalene plane respectively. The twist in the naphthalene backbone is quite pronounced, and is exhibited by the angle between the $C(1)-C(5)$ and $C(6)-C(10)$ planes of $5.77(6)^{\circ}$. This is a significantly twisted structure compared to complexes $\mathbf{V I}$ and other diaminonaphthalene based ligands, in which the naphthalene ring is normally completely planar as would be expected of and $\mathrm{sp}^{2}$ hybridised system. ${ }^{66-68}$ Complex III(d) has similar Ge$\mathrm{N}$ bond distances of 1.866 and $1.862 \AA$, indicating the size of the carbon backbone has little influence on the ligands coordination to germanium. ${ }^{23} 3$ has longer $\mathrm{N}$-Si bond lengths than VI of 1.842 and $1.841 \AA$. The change in Ge-N bond length when comparing $N-{ }^{i} \operatorname{Pr}$ to $N$-TMS likely results from the increased steric bulk associated with the TMS group over the 'Pr group, as well as the difference in electronics due to the lower electronegativity of Si over $\mathrm{C}$ resulting in more electron density on the nitrogen atoms and shorter Ge-N bond distance. The solid-state structure of $\mathbf{3}$ differs significantly to the compound [Sn(NAPH $\left.{ }^{\mathrm{TMS}}\right)$ ] $(\mathrm{XL})$, in which the tin atom sits above the plane of the naphthalene ring with the N-TMS groups sitting below the plane. ${ }^{66}$

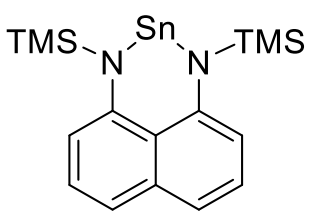

XL

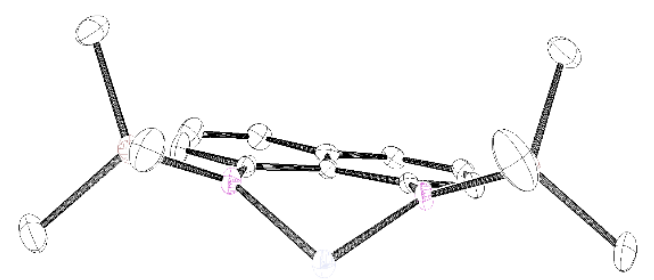

Figure 15: ORTEP representation of $X L$, showing out of the NCCCN plane tin atom.

This indicates that the tin(II) cannot fit into the bonding pocket of the ligand plane, presumably due to the larger ionic radii on $\mathrm{Sn}$ (II) over Ge(II). However, it is interesting that in the solid-state structure of $\mathbf{3}$ the 
naphthalene backbone twists to accommodate the germanium atom, opposed to being isostructural with $\mathbf{X L}$ with the metal atom sitting above the plane of the naphthalene backbone, as the large extended $\pi$ system of the [NAPH ${ }^{\mathrm{TMS}}$ ] would be expected to result in a linear naphthalene backbone, with a high energy barrier to the conformational twisting required to fit $\mathrm{Ge}(\mathrm{II})$ in plane.

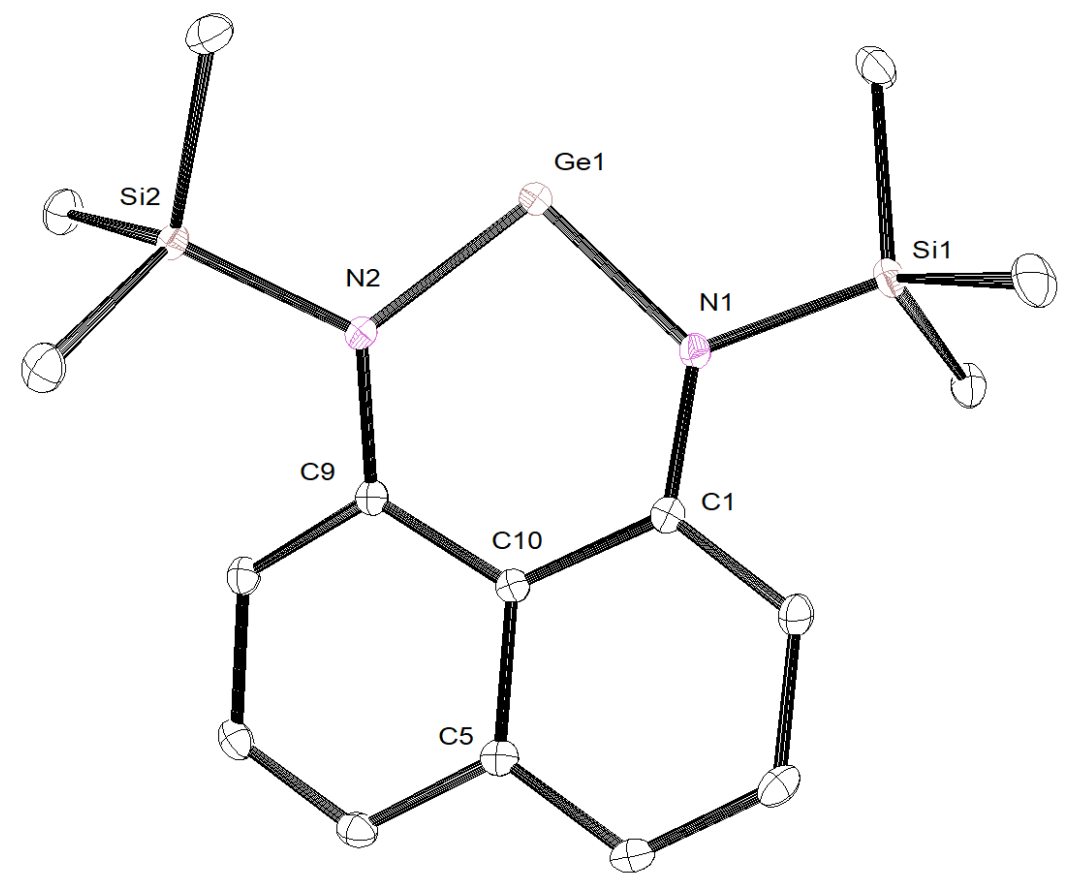

Figure 16: ORTEP representation of 3. $30 \%$ Thermal ellipsoids. Hydrogens omitted for clarity.

Table 3: Selected bond lengths and angles of 3.

Bond Lengths ( $\AA$ )

$\begin{array}{cc}\mathrm{Ge}(1)-\mathrm{N}(1) & 1.8641(16) \\ \mathrm{Ge}(1)-\mathrm{N}(2) & 1.8577(16) \\ \mathrm{N}(1)-\mathrm{C}(1) & 1.413(2) \\ \mathrm{N}(2)-\mathrm{C}(9) & 1.405(2)\end{array}$

Bond Angles ( ${ }^{\circ}$ )

$\begin{array}{lc}N(1)-G e(1)-N(2) & 98.11(7) \\ \text { Ge(1)-N(1)-Si(1) } & 114.67(10) \\ \text { Ge(1)-N(2)-Si(2) } & 116.34(10) \\ \text { Ge(1)-N(2)-C(9) } & 123.34(12) \\ \text { Ge(1)-N(1)-C(1) } & 124.31(12) \\ N(1)-C(1)-C(10) & 121.80(15) \\ N(2)-C(9)-C(10) & 121.51(15)\end{array}$




\subsubsection{Attempted synthesis of $\left[\mathrm{Pb}\left(\mathrm{NAPH}^{\mathrm{TMS}}\right)\right]$}

The synthesis of $\left[\mathrm{Pb}\left(\mathrm{NAPH}^{\mathrm{TMS}}\right)\right]$ was also attempted from using the same synthetic route that was successful for 3. After workup, a yellow solution was observed to change over a number of days to give a vibrant dark green solution, identified as $\mathbf{X L I}$ by single crystal X-ray diffraction and ${ }^{1} \mathrm{H}$ NMR spectroscopy. $\mathbf{X L I}$ has been previously synthesised from the decomposition of the thallium compound $\left[\mathrm{TI}_{2}\left(\mathrm{NAPH}^{\mathrm{TMS}}\right)\right]$ at $90{ }^{\circ} \mathrm{C} .{ }^{69}$ It is presumed that, like the thalium compound, the lead complex [Pb(NAPH $\left.{ }^{\mathrm{TMS}}\right)$ ] also undergoes demetallation to give $\mathbf{X L I}$ and a black solid assumed to be elemental lead. Our results suggest that the lead complex is a better synthetic route to $\mathrm{XLI}$, as, unlike the thalium complex, the solution gave $\mathrm{XLI}$ at $-30{ }^{\circ} \mathrm{C}$ (Scheme 17).

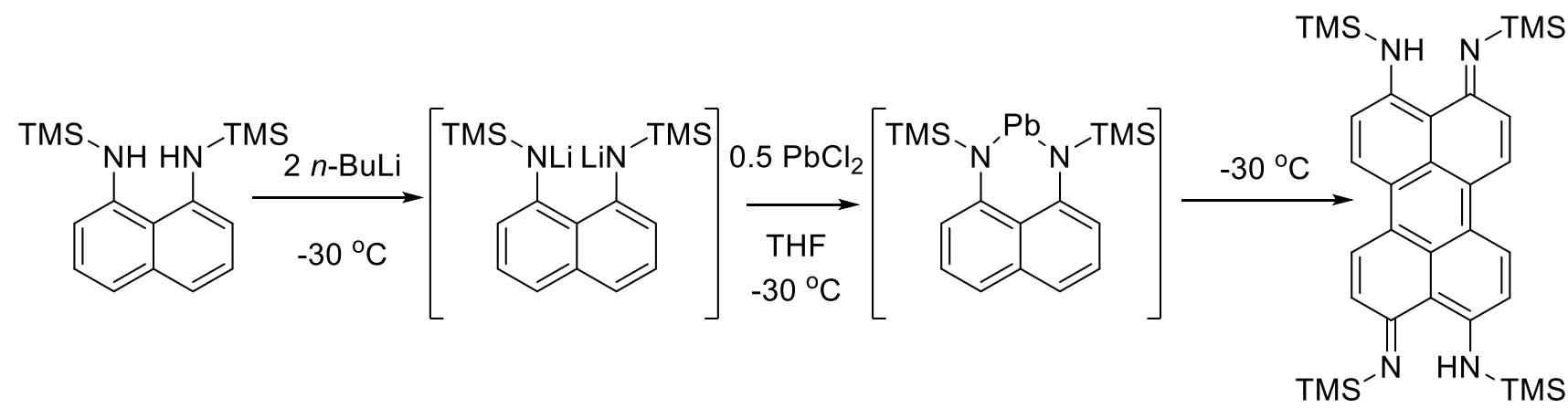

Scheme 17: Synthesis of $\mathrm{XLI}$ from attempted synthesis of [Pb(NAPH $\left.\left.{ }^{\mathrm{TMS}}\right)\right]$.

$X \mathrm{XI}$

\subsection{DFT Analysis of Group 14 Structures}

To further investigate the structure, bonding and potential reactivity of the synthesised germylenes relative to their heavier group 14 analogues $(\mathrm{Sn}, \mathrm{Pb}$ ) geometry optimisations and molecular orbital (MO) density functional theory (DFT) calculations were carried out. Structures were optimized in the PBE1PBE/def2SVP level of theory for geometry optimisations and PBE1PBE/def2TZVP level of theory for molecular orbital calculations.

\subsection{1 [Ge(NAPH $\left.\left.{ }^{\mathrm{TMS}}\right)\right]$}

The geometry optimisation of $\mathbf{2}$ gave bond lengths that agree well with the bond lengths of the solid-state crystal structure data. Other calculated computational information such as stretching frequencies, partial 
charges and molecular orbitals can provide useful insight about the structure, bonding and reactivity of inorganic complexes and will therefore be discussed.

Table 4: Crystal structure bond lengths. Computational bond lengths in brackets. The NCN plane is defined as the plane of the nitrogen atom with the carbon atom $\beta$ to both nitrogen atoms.

$\begin{array}{crcccc} & {\left[\mathrm{Ge}\left(\mathrm{NAPH}^{\mathrm{TMS}}\right)\right]} & {\left[\mathrm{Ge}\left(\mathrm{NAPH}^{\mathrm{TMS}}\right)\right]} & {\left[\mathrm{Sn}\left(\mathrm{NAPH}^{\mathrm{TMS}}\right)(\mathrm{XL})\right]} & {\left[\mathrm{Sn}\left(\mathrm{NAPH}^{\mathrm{TMS}}\right)\right]} & {\left[\mathrm{Pb}\left(\mathrm{NAPH}^{\mathrm{TMS}}\right)\right]} \\ \mathbf{M}-\mathbf{N}(\AA ̊) & 1.864 & {[1.863]} & 2.100 & {[2.078]} & {[2.186]} \\ & 1.858 & {[1.863]} & 2.100 & {[2.078]} & {[2.186]} \\ \mathbf{N}-\mathbf{C}(\AA ̊) & 1.413 & {[1.404]} & 1.383 & {[1.402]} & {[1.398]} \\ & 1.405 & {[1.404]} & 1.390 & {[1.402]} & {[1.398]} \\ \mathbf{N}-S i(\AA ̊) & 1.772 & {[1.797]} & 1.735 & {[1.782]} & {[1.766]} \\ & 1.770 & {[1.979]} & 1.746 & {[1.782]} & {[1.766]} \\ \text { C-C average (Å) } & 1.405 & {[1.408]} & 1.405 & {[1.409]} & {[1.411]} \\ \text { M-NCN Plane } & 4.31^{\circ} & {\left[0.01^{\circ}\right]} & 35.26^{\circ} & {\left[0.01^{\circ}\right]} & {\left[29.35^{\circ}\right]}\end{array}$

Table 4 compares bond lengths for gas phase optimised structures and the crystal structure data (either obtained or literature values). The solid-state bond distances agree well with the theoretical gas-phase bond distances. Also included is the angle between the metal atom and the plane of the two nitrogen atoms and the $\beta$ carbon atom to these nitrogen atoms (the NCN plane). This was included as in the crystal structure data it was noted that as progresses down group 14 the metal atom sits further out of plane with the NCN atoms. This is likely due to the increasing ionic radii of the metal ions $\left(\mathrm{Ge}^{2+} 87<\mathrm{Sn}^{2+} 118\right.$ $<\mathrm{Pb}^{2+} 133 \mathrm{pm}$ ) resulting in the binding pocket being too small. Interestingly, the DFT calculations only expect this out of plane conformation for the lead atom, but the crystal structure data clearly shows that in the solid-state structure of the tin complex $\mathbf{X L}$, this out of plane effect is pronounced. 


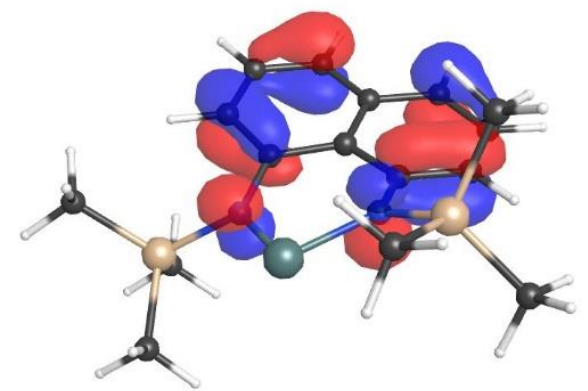

HOMO

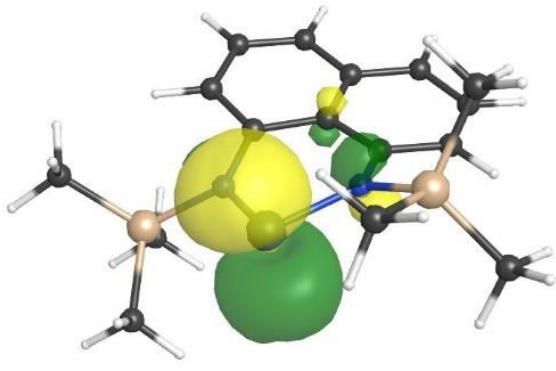

LUMO

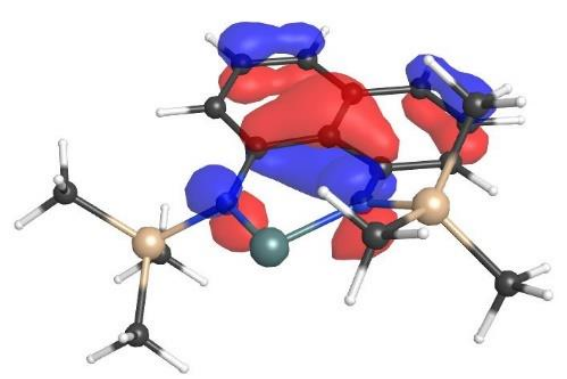

HOMO-1

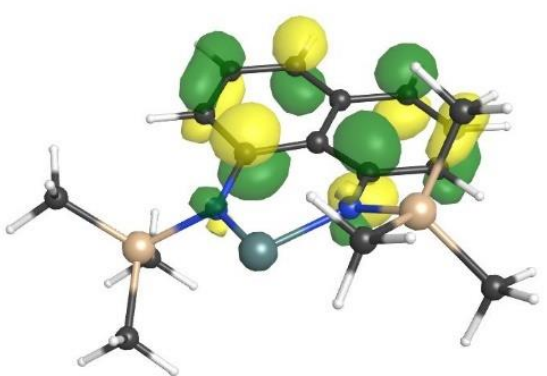

LUMO+1

Figure 17: Frontier molecular orbitals of [Ge(NAPH $\left.{ }^{\mathrm{TMS}}\right]$ ].

To gain more insight into the trend of potential reactivity for the heavier group 14 structures molecular orbital (MO) calculations were carried out to compare the energy gap of the frontier molecular orbitals. The molecular orbital calculations were completed using the PBE1PBE/def2TZVP level of theory on the geometry optimized gas-phase structures. The MOs for [Ge(NAPH $\left.\left.{ }^{\mathrm{TMS}}\right)\right]$ are depicted in Figure 17. The HOMO of the complex appears to be strongly located on the $\pi$ system of the naphthalene ring with density delocalized onto the nitrogen atoms. The LUMO has a major contribution from the empty $p$ orbital, while the LUMO+1 has the electron density located both on the $\pi$ system of the naphthalene ring and as a node on the germanium atom. The HOMO-LUMO energy gap, used commonly as an indicator for potential reactivity of inorganic complexes was calculated to be $354 \mathrm{~kJ} \mathrm{~mol}^{-1}(3.67 \mathrm{eV})$. This is a relatively large energy gap for a germylene and would indicate that the complex is likely unreactive towards a range of small molecules. 


\subsection{2 [Sn(NAPH $\left.\left.{ }^{\mathrm{TMS}}\right)\right]$}

The frontier molecular orbitals for $\left[\mathrm{Sn}\left(\mathrm{NAPH}^{\mathrm{TMS}}\right)\right]$ ( $\mathrm{XL}$ ) show similar areas of electron density as the [Ge(NAPH $\left.{ }^{\mathrm{TMS}}\right)$ ], with the HOMO electron density being located on the $\pi$ system of the naphthalene ring, while the LUMO electron density is mainly located on the empty $p$ orbirtal of the tin atom, with a small amount of electron density on the $p$ orbitals of the nitrogen atoms.

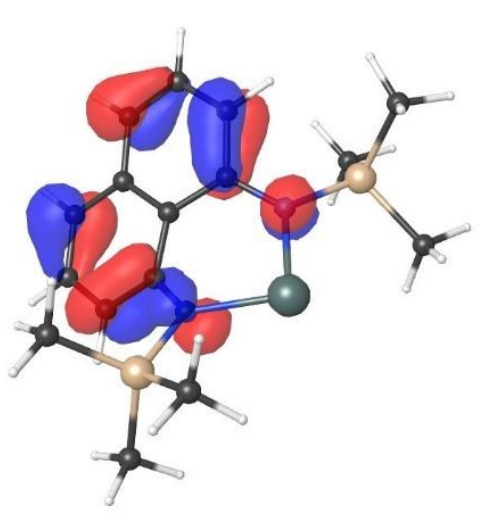

HOMO

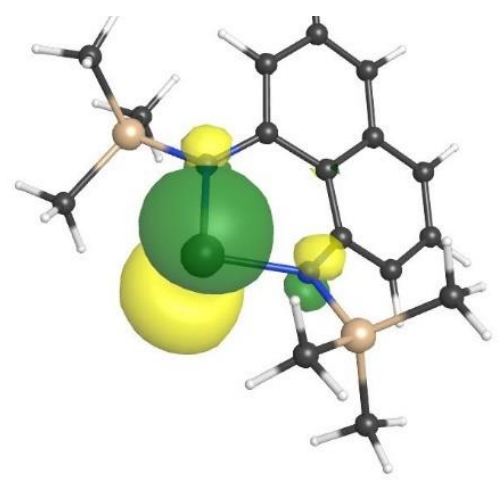

LUMO

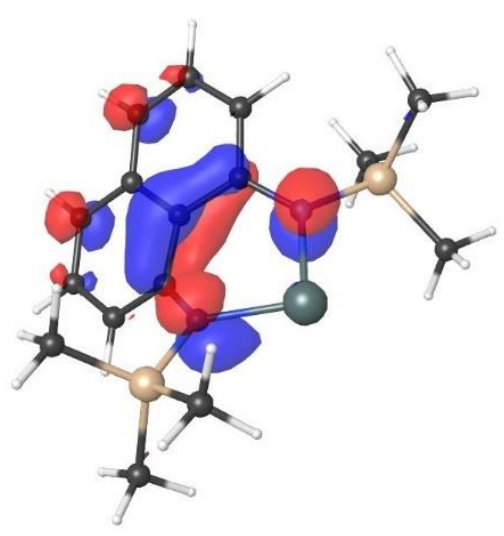

HOMO-1

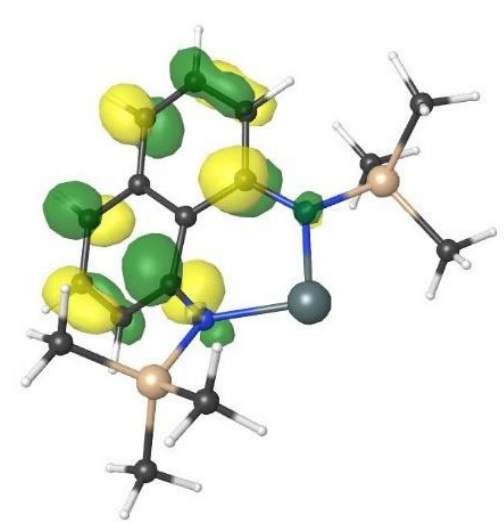

LUMO+1

Figure 18: Frontier molecular orbitals of [Sn(NAPH $\left.\left.{ }^{\mathrm{TMS}}\right)\right]$.

The orbital size the empty p orbital electron density of the LUMO has increased relative to $\mathbf{3}$, though this effect was not quantified using NBO calculations due to time constraints. The HOMO-LUMO energy gap was calculated to be $294 \mathrm{kJmol}^{-1}(3.05 \mathrm{eV}), 60 \mathrm{~kJ} \mathrm{~mol}^{-1}$ lower than for the germylene complex, indicating [Sn(NAPH $\left.\left.{ }^{\mathrm{TMS}}\right)\right]$ would be more reactive towards a range of small molecules. 


\subsection{3 [Pb(NAPH $\left.\left.{ }^{\mathrm{TMS}}\right)\right]$}

For completeness, the frontier molecular orbitals were also calculated for the complex $\left[\mathrm{Pb}\left(\mathrm{NAPH}{ }^{\mathrm{TMS}}\right)\right]$. The results showed the continued trend as group 14 is descended; the molecular orbitals have the electron density located on the $\pi$ naphthalene ring and the nitrogen atoms in the HOMO, while the LUMO has the majority of the electron density located in the empty $p$ orbital on the lead atom.

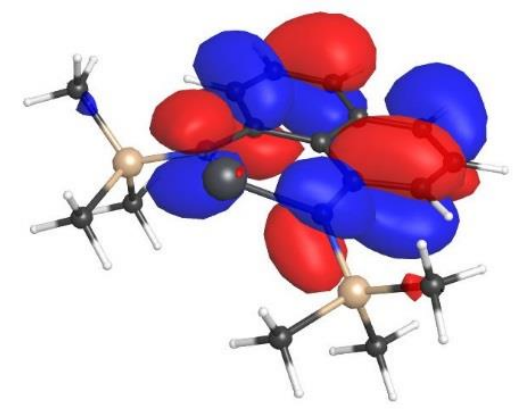

HOMO

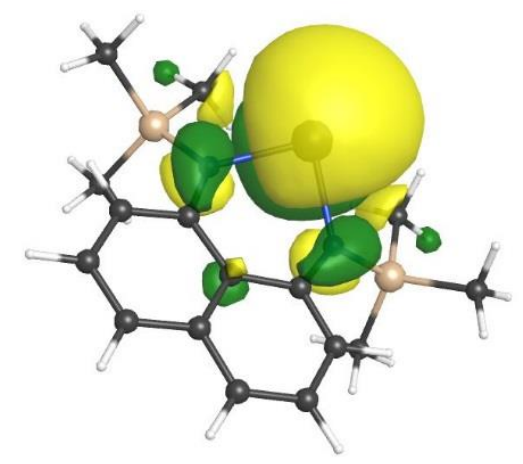

LUMO

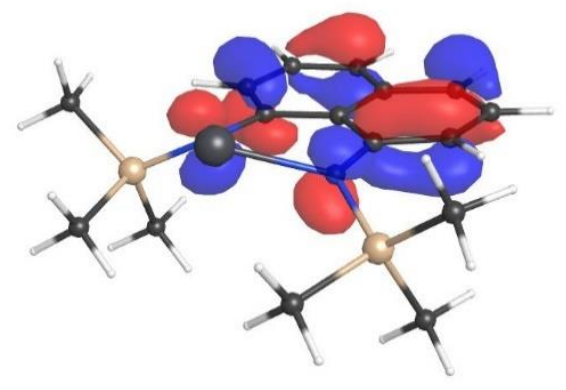

HOMO-1

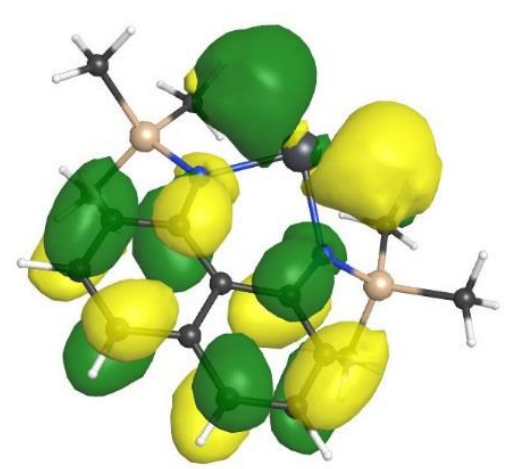

LUMO+1

Figure 19: Frontier molecular orbitals of $\left[\mathrm{Pb}\left(\mathrm{NAPH}^{\mathrm{TMS}}\right)\right]$.

The summary of the MO calculations is summarised in Table 5 and Figure 19. The data shows that as group 14 descends the energy of the LUMO decreases while the HOMO increases in energy, decreasing the HOMO-LUMO energy gap. This would be expected to result in a higher reactivity for the heavier [NAPH ${ }^{\mathrm{TMS}}$ ] compounds. 
Table 5: Relative orbital energies calculated for $3, \mathrm{XL}$ and [Pb(NAPH $\left.\left.{ }^{\mathrm{TMS}}\right)\right]$.

\begin{tabular}{|c|c|c|c|}
\hline & {$\left[\mathrm{Ge}\left(\mathrm{NAPH} \mathrm{TMS}^{\mathrm{TMS}}\right)\right]$} & {$\left[\mathrm{Sn}\left(\mathrm{NAPH}{ }^{\mathrm{TMS}}\right)\right]$} & {$\left[\mathrm{Pb}\left(\mathrm{NAPH}^{\mathrm{TMS}}\right)\right]$} \\
\hline HOMO-1 (kJ mol-1) & -656.36 & -624.85 & -588.10 \\
\hline HOMO(kJ mol-1) & -496.21 & -483.08 & -467.33 \\
\hline LUMO(kJ mol-1) & -141.77 & -189.03 & -212.66 \\
\hline LUMO+1 (kJ mol-1) & -76.14 & -68.26 & -76.14 \\
\hline HOMO-LUMO (kJ mol-1) & 354.43 & 294.05 & 254.67 \\
\hline HOMO-LUMO (eV) & 3.67 & 3.05 & 2.64 \\
\hline
\end{tabular}

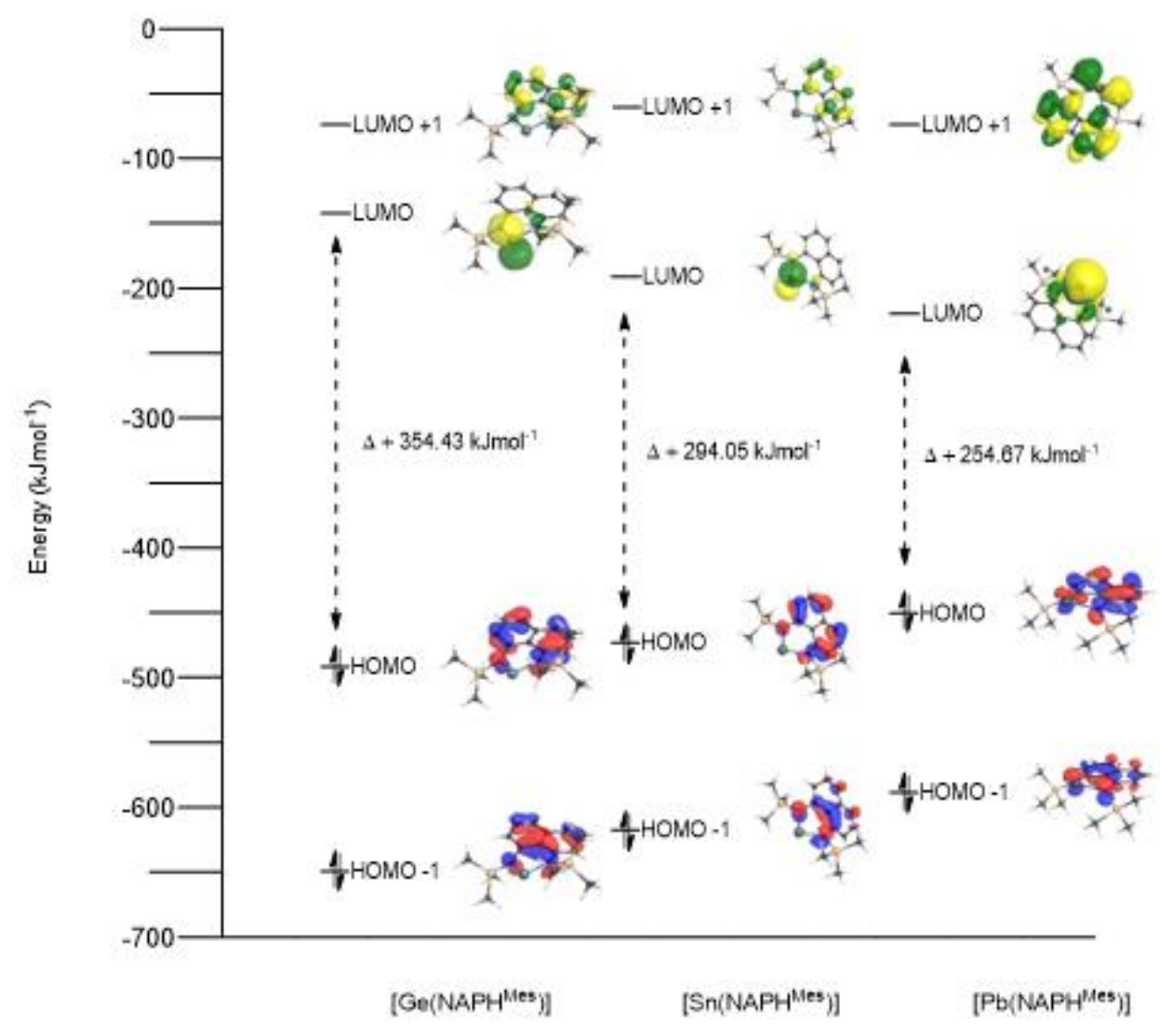

Figure 20: Diagram showing energy of frontier MO's for [NAPH ${ }^{\mathrm{TMS}}$ ] compounds. 
For a comparison, the [BIAN ${ }^{\text {Mes }}$ ] heavier group 14 compounds ( $\mathrm{Ge}, \mathrm{Sn}, \mathrm{Pb}$ ) were also investigated computationally to gain insight into their bonding and reactivity. A comparison with the crystal structure data can only be made for [(GeBIAN $\left.\left.{ }^{\text {Mes }}\right)\right]$, as the other two complexes ([Sn(BIAN $\left.\left.{ }^{M e s}\right)\right]$ and $\left[\mathrm{Pb}\left(\mathrm{BIAN}^{\mathrm{Mes}}\right)\right]$ ) were not able to be synthesised. Unlike the group 14 compounds supported with the [NAPH ${ }^{\mathrm{TMS}}$ ] ligand, all three of the optimised structures for the [BIAN ${ }^{\text {Mes }}$ ligand predict that the metal atom will sit in the plane of the $\mathrm{N}-\mathrm{C}=\mathrm{C}-\mathrm{N}$ ligand backbone. This is surprising when comparing the results to the [NAPH ${ }^{\mathrm{TMS}}$ ] optimisations, as [BIAN ${ }^{\mathrm{Mes}}$ ] would be expected to have a smaller binding pocket. The data suggests that as the metal atom ionic radii increases the metal to nitrogen bond length increases, the lengthening of this bond paired with the decreasing electronegativity as group 14 descends results in a shortening of the $\mathrm{N}-\mathrm{C}$ bond lengths to both the carbon backbone of the ligand and to the mesityl groups. This could be due to the significant increase in negative charge located on the nitrogen atoms for the tin and lead complexes, likely due to the significantly lower electronegativity for these metals compared to germanium. ${ }^{70}$

Table 6: Crystal structure of selected bond lengths compared to computationally derived optimized bond lengths.

\begin{tabular}{|c|c|c|c|c|}
\hline & {$\left[\mathrm{Ge}\left(\mathrm{BIAN} \mathrm{Nes}^{\mathrm{Mes}}\right)\right](2)$} & {$\left[\mathrm{Ge}\left(\mathrm{BIAN} \mathrm{Nes}^{\mathrm{Mes}}\right)\right]$} & {$\left[\mathrm{Sn}\left(\mathrm{BIAN}{ }^{\mathrm{Mes}}\right)\right]$} & {$\left[\mathrm{Pb}\left(\mathrm{BIAN} \mathrm{Nes}^{\mathrm{Mes}}\right)\right]$} \\
\hline \multirow[t]{2}{*}{ M-N (Å) } & 1.891 & [1.905] & [2.130] & {$[2.240]$} \\
\hline & 1.889 & [1.905] & [2.130] & {$[2.240]$} \\
\hline \multirow[t]{2}{*}{ N-C (A) } & 1.375 & [1.365] & [1.359] & [1.352] \\
\hline & 1.371 & [1.365] & [1.359] & [1.352] \\
\hline \multirow[t]{2}{*}{ N-C(Mes) (Å) } & 1.429 & [1.422] & [1.419] & {$[1.415]$} \\
\hline & 1.431 & [1.422] & [1.419] & [1.415] \\
\hline$C=C(\AA)$ & 1.379 & [1.386] & [1.398] & {$[1.406]$} \\
\hline $\mathrm{M}-\mathrm{NC}=\mathrm{CN}$ Plane $\left(^{\circ}\right)$ & 0.32 & {$[0.01]$} & {$[0.01]$} & {$[0.00]$} \\
\hline Partial Charge on Metal & & [0.145] & [0.405] & [0.408] \\
\hline Partial Charge on $\mathrm{N}$ & & {$[0.060]$} & {$[-0.279]$} & {$[-0.278]$} \\
\hline
\end{tabular}




\subsection{4 $\left[\mathrm{Ge}\left(\mathrm{BIAN}^{\mathrm{Mes}}\right)\right]$}

The calculated frontier molecular orbitals of [Ge(BIAN $\left.\left.{ }^{M e s}\right)\right]$ show the electron density of the HOMO sits in the $\pi$ system of the 5 -membered heterocyclic ring, with a slightly larger coefficient of electron density on the germanium atom relative to the rest of the heterocyclic ring. The LUMO has the electron density calculated as occupying the $\pi$ system of the naphthalene based carbon backbone of the ligand, with no electron density on either the germanium atom or the mesityl groups. The LUMO+1 orbitals almost exclusively have the electron density on an empty $\mathrm{p}$ orbital of the germanium atom. The calculated HOMO-LUMO gap for [(Ge(BIAN $\left.\left.{ }^{M e s}\right)\right]$ is $\left.304.55 \mathrm{kJmol}^{-1}, 3.16 \mathrm{eV}\right)$ which indicates that the energy barrier to reactivity is smaller for $\left[\left(\mathrm{Ge}\left(\mathrm{BIAN}^{\mathrm{Mes}}\right)\right]\right.$ than $\left[\mathrm{Ge}\left(\mathrm{NAPH}^{\mathrm{TMS}}\right)\right]$. This, however, does not consider any steric activity on reaction rate, so cannot be thought of as a definitive indication of reactivity.

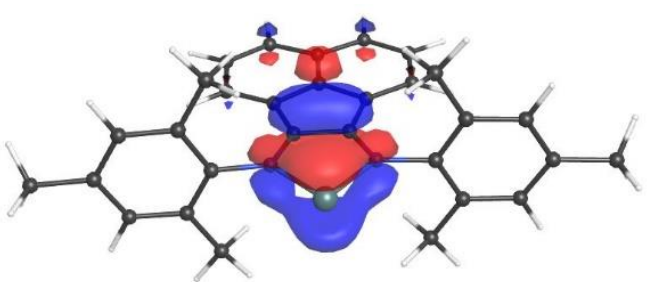

HOMO

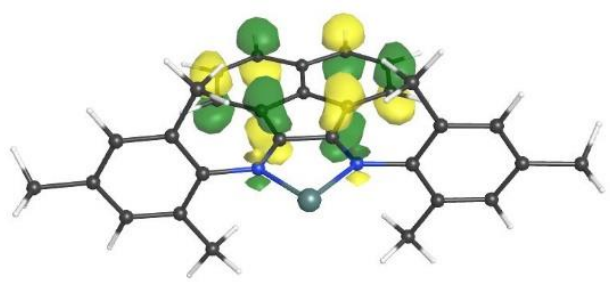

LUMO

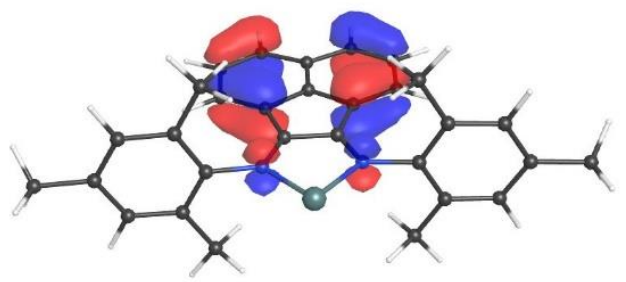

HOMO-1

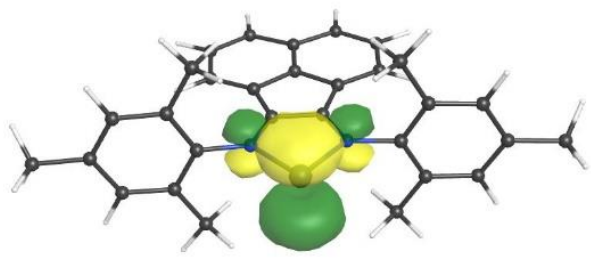

LUMO+1

Figure 21: Frontier molecular orbitals for [Ge(BIAN $\left.\left.{ }^{\mathrm{Mes}}\right)\right]$.

\subsection{5 [Sn(BIAN $\left.\left.{ }^{\text {Mes }}\right)\right]$}

The gas phase geometry optimisation shows the mesityl groups on the nitrogen atoms sit orthogonal to the plane of the $\mathrm{sp}^{2}$ hybridised carbon backbone of the ligand. This compound probably exists in this conformation to minimise steric repulsion between the methyls in the 2,6 positions of the mesityl group 
with the tin atom and with the carbons of the $\mathrm{sp}^{2}$ hybridised carbon backbone of the ligand. The frontier molecular orbitals of [Sn(BIAN $\left.{ }^{\text {Mes }}\right)$ ] show the electron density of the HOMO located on the $\pi$ system of the tin, nitrogen and $\alpha$-carbon atoms, while the LUMO orbital electron density is located on the $\pi$ system of the $\mathrm{sp}^{2}$ hybridised carbon backbone. The HOMO energy of [Sn(BIAN $\left.{ }^{\mathrm{Mes}}\right)$ ] is $-446.33 \mathrm{~kJ} \mathrm{~mol}^{-1}$, slightly higher energy than the complex [Sn(NAPH $\left.\left.{ }^{\text {TMS }}\right)\right]\left(-483.08 \mathrm{~kJ} \mathrm{~mol}^{-1}\right)$. The LUMO energy of $-162.78 \mathrm{~kJ} \mathrm{~mol}^{-1}$ is also higher energy than [Sn(NAPH ${ }^{\text {TMS }}$ )], but the actual HOMO-LUMO energy gap (283.55 $\mathrm{kJmol}^{-1}$ ) is less than the $\left[\mathrm{Sn}\left(\mathrm{NAPH}^{\mathrm{TMS}}\right)\right]\left(294.05 \mathrm{kJmol}^{-1}\right)$. This indicates that the [Sn(BIAN $\left.\left.{ }^{\mathrm{Mes}}\right)\right]$ complex would likely be more reactive towards small molecules than the complex [Sn(NAPH $\left.{ }^{\mathrm{TMS}}\right)$ ]. The LUMO orbital electron density is located on the $\pi$ system of the naphthalene ring and the $\alpha$ carbons bonded to the nitrogen atoms. The electron density of the LUMO+1 is located on a p orbital of the tin atom, the same as the germanium analogue.

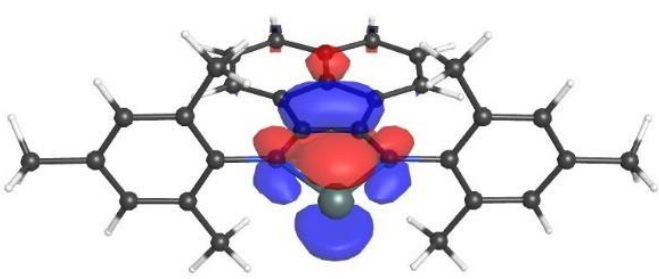

HOMO

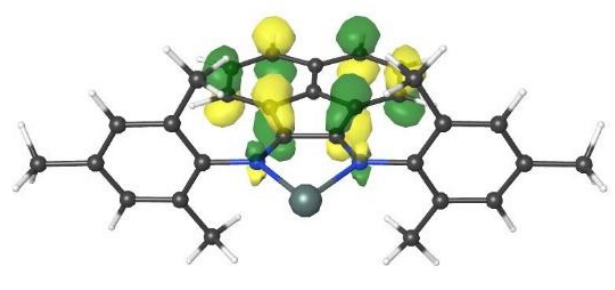

LUMO

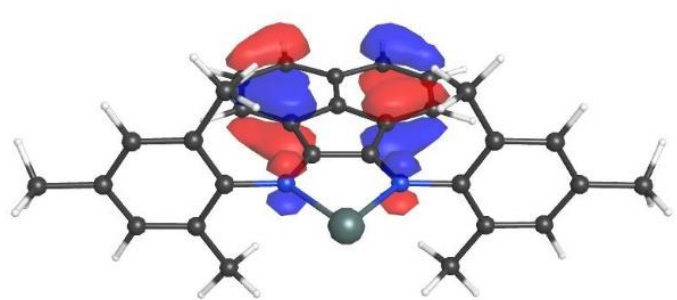

HOMO-1

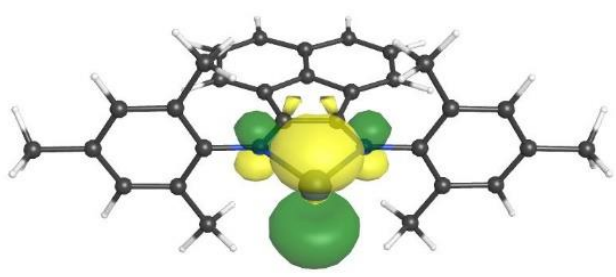

LUMO+1

Figure 22: Frontier molecular orbitals for [Sn(BIAN $\left.\left.{ }^{\text {Mes }}\right)\right]$.

\subsection{6 $\left[\mathrm{Pb}\left(\mathrm{BIAN}^{\mathrm{Mes}}\right)\right]$}

The gas phase geometry optimisation of $\left[\mathrm{Pb}\left(\mathrm{BIAN}^{\mathrm{Mes}}\right)\right]$ showed the same conformation as the germanium and tin analogues with the mesityl plane orthogonal to the plane of the $\mathrm{sp}^{2}$ hybridised backbone. The metal to nitrogen bond lengths lengthen, as would be expected due to the increased ionic radii of $\mathrm{Pb}$ (II) meaning it will sit further outside the binding pocket relative to the $\mathrm{Ge}(\mathrm{II})$ and $\mathrm{Sn}(\mathrm{II})$. The length of the $\mathrm{N}$ $\mathrm{C}$ of the $\mathrm{sp}^{2}$ hybridized system shortens from 1.359 to $1.352 \AA$ from $\mathrm{Sn}$ to $\mathrm{Pb}$, while the $\mathrm{C}-\mathrm{C}$ (Mes) bond 
also shortens from 1.419 to $1.415 \AA$. This could be due to the decreased electron density on nitrogen as group 14 is descended, as is evident from the partial charges on the nitrogen atom going from $\mathrm{Ge}$ to $\mathrm{Pb}$ (Table 7).

The frontier molecular orbitals for $\left[\mathrm{Pb}\left(\mathrm{BIAN}^{\mathrm{Mes}}\right)\right]$ show a significant difference in the LUMO electron density from the lighter two group 14 analogues. For the Ge and Sn complexes the LUMO electron density was located almost entirely on the $\pi$ system of the carbon backbone of the ligand, with the LUMO+1 having its electron density located on the metal $\pi$ orbital of the metal. For $\mathrm{Pb}$ these molecular orbitals are reversed, with the LUMO of the $\mathrm{Pb}$ complex being lower in energy than the increasing energy of the MO relating to the $\pi$ system of the carbon backbone of the ligand (Table 7). This implies that as group 14 descends, this orbital becomes a more energetically favourable state. The partial positive charge on the metal atom of the complexes can also be seen to increase as group 14 descends. This could be a function of either the decreasing electron density of the metal ions, although due to the increasing inert pair effect as the group descends it would be expected that the partial charge on the metal might decrease as more electron density is located on the metal (and therefore not in bonding MO bonding combinations).

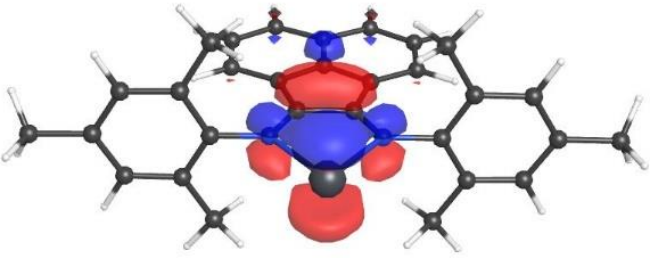

HOMO

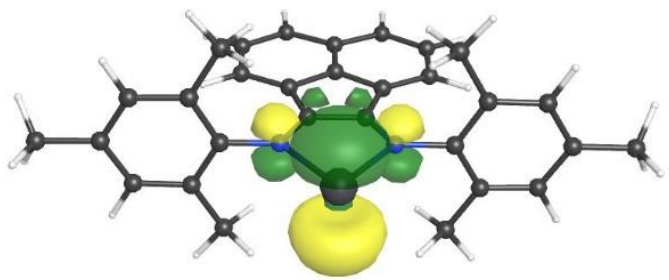

LUMO

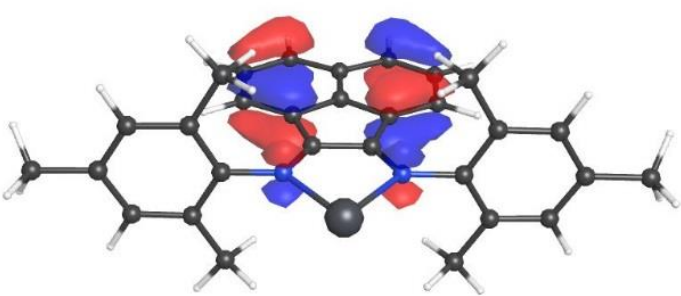

HOMO-1

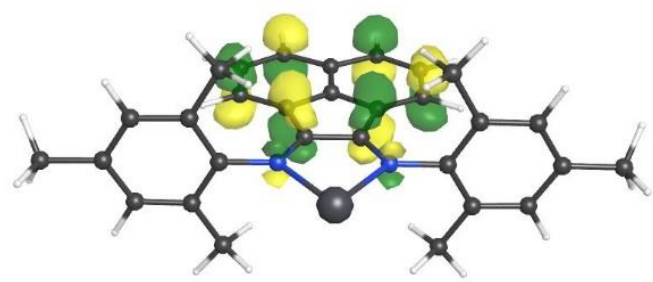

LUMO+1

Figure 23: Frontier Molecular orbitals for [Pb(BIAN $\left.\left.{ }^{\mathrm{Mes}}\right)\right]$. 
The summary of the $\mathrm{MO}$ calculations for the [BIAN $\left.{ }^{\text {Mes }}\right]$ complexes is summarised in Table 7 and Figure 24. The data shows that as group 14 descends the energy of the LUMO does not significantly change while the HOMO increases in energy, decreasing the HOMO-LUMO energy gap. This would be expected to result in a higher reactivity for the heavier [BIAN $\left.{ }^{\mathrm{Mes}}\right]$ compounds.

Table 7: Crystal structure of selected bond lengths compared to computationally derived optimised bond lengths.

\begin{tabular}{|c|c|c|c|}
\hline & [Ge(BIAN $\left.\left.{ }^{\text {Mes }}\right)\right]$ & [Sn(BIAN $\left.\left.{ }^{\text {Mes }}\right)\right]$ & {$\left[\mathrm{Pb}\left(\mathrm{BIAN}^{\mathrm{Mes}}\right)\right]$} \\
\hline HOMO-1 (kJ mol-1) & -614.35 & -603.85 & -595.97 \\
\hline HOMO(kJ mol'-1) & -475.20 & -446.32 & -425.32 \\
\hline LUMO(kJ mol-1) & -170.65 & -162.78 & -168.03 \\
\hline LUMO+1 (kJ mol-1) & -118.14 & -160.15 & -154.90 \\
\hline HOMO-LUMO (kJ mol-1) & 304.55 & 283.55 & 257.29 \\
\hline HOMO-LUMO (eV) & 3.16 & 2.94 & 2.67 \\
\hline
\end{tabular}

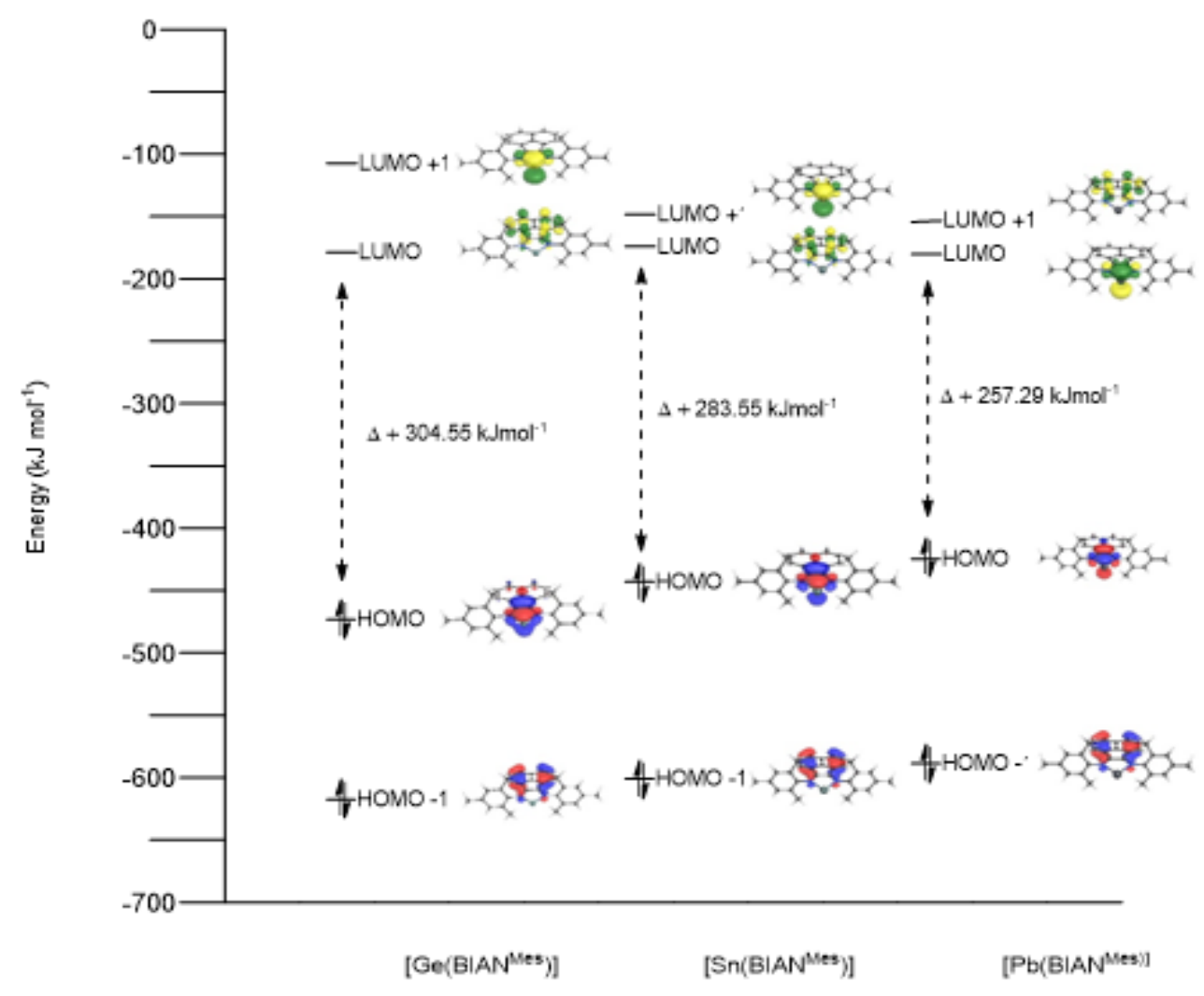

Figure 24: Diagram showing energy of frontier MO's for [BIAN"Mes] compounds. 


\subsection{Mixed Coordination Germanium Species:}

The anionic species [Ge(NAPH $\left.\left.{ }^{\mathrm{TMS}}\right) \mathrm{Ge}(\mathrm{Bu})\left(\mathrm{NAPH}^{\mathrm{TMS}}\right)\right]^{-} \mathbf{4}$ and $\left[\mathrm{Ge}\left(\mathrm{NAPH}^{\mathrm{TMS}}\right) \mathrm{Ge}(\mathrm{Me})\left(\mathrm{NAPH}^{\mathrm{TMS}}\right)\right]^{-} \mathbf{5}$ have been synthesised by the addition of $n$-BuLi or MeLi to $\mathbf{3}$ in THF. In both $\mathbf{4}$ and $\mathbf{5}$ the overall negative charge of the species appears to be located of the three-coordinate germanium in an interesting donor-acceptor type relationship. The complexes were originally synthesised accidentally during the synthesis of $\mathbf{3}$, when the $\left[\mathrm{NAPH}^{\mathrm{TMS}}-\mathrm{H}\right.$ ] preligand was lithiated in situ before its addition to $\mathrm{GeCl}_{2}$.dioxane. Residual $n$-BuLi in the reaction reacted with $\mathbf{3}$, resulting in the formation of $\mathbf{4}$. Complex $\mathbf{4}$ and $\mathbf{5}$ were more easily synthesised from the addition of half a molar equivalent of either $n$-BuLi or MeLi to clean samples of 3. Although the complexes are not relevant to the synthesis of the desired heterobimetallic $\mathbf{1}$, the complexes are reported here as they have unique structure and bonding properties, as well as giving some insight into the reactivity of germylene 3 .

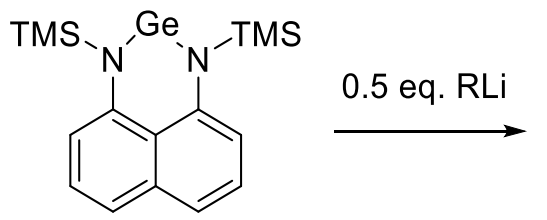

3

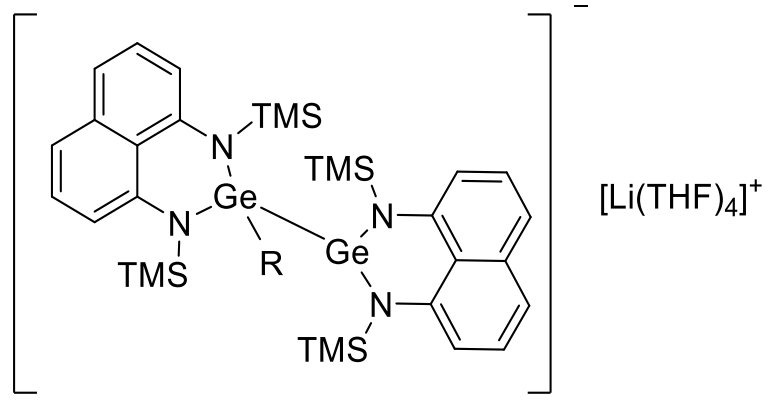

$4 ; \mathrm{R}=n-\mathrm{Bu}$

5; $\mathrm{R}=\mathrm{Me}$

\subsection{1 $\left[\mathrm{Ge}\left(\mathrm{NAPH}^{\mathrm{TMS}}\right) \mathrm{Ge}(\mathrm{Bu})\left(\mathrm{NAPH}^{\mathrm{TMS}}\right)\right]^{-[}\left[\mathrm{Li}\left(\mathrm{THF}_{4}\right)\right]^{+} \quad 4$}

The ${ }^{1} \mathrm{H}$ NMR spectrum for $\mathbf{4}$ displayed many new resonances compared to the free germylene $\mathbf{3}$. The most significant of these resonances are the high field signals attributed to the incoming alkyl fragment ( $\delta$ 1.19$1.14,0.75,0.50 \mathrm{ppm})$, which would be expected to show four resonances; however, the signal at $\delta 0.50$ ppm appears to be two overlapping signals, as is evident from its integration (four protons). The ${ }^{1} \mathrm{H}$ NMR spectrum also has two new resonances attributed to the two different TMS environments in the high field $(\delta 0.54,0.44 \mathrm{ppm})$, both of which integrate to 18 protons. The resonances at $\delta 3.29$ and $1.23 \mathrm{ppm}$ are attributed to the four THF molecules solvating the lithium ion (also confirmed by the solid-state crystal structure), but it should be noted that these shifts are upfield shifted from free THF in $\mathrm{C}_{6} \mathrm{D}_{6}$ resonances ( $\delta$ 3.57 and $1.40 \mathrm{ppm}$ respectively) which would be expected due to the electronic influence of the lithium ion. ${ }^{71}$ 
Crystals suitable for an X-ray diffraction study were grown from a concentrated solution of $\mathbf{4}$ in THF. The solid-state crystal structure data of $\mathbf{3}$ shows the $\mathrm{Ge}(1)$ in a distorted trigonal pyramidal arrangement with three coordinating atoms while $\mathrm{Ge}(2)$ is in a distorted tetrahedral arrangement (Figure 25). The Ge(1)Ge(2) bond length of $2.6222 \AA$ is longer than that of XXV (Ge-Ge 2.611 ̊), shorter than XXVII (Ge-Ge 2.881 $\AA$ ) , and significantly longer than the sum of two Ge covalent radii $\left(2.44 \AA\right.$ ).${ }^{45-46,72}$ The asymmetric unit cell of 4 shows 1 molecule with a 4 coordinate tetrahedral germanium $(\mathrm{Ge}(2))$ supported by the N-heterocyclic [NAPH ${ }^{\mathrm{TMS}}$ ] ligand and an alkyl chain, and a second 3 coordinate germanium (Ge(1)) atom supported with the same $\mathrm{N}$-heterocyclic [NAPH ${ }^{\mathrm{TMS}}$ ] ligand. A THF solvated lithium ion balances the charge of the negatively charged germanium to make the unit cell a neutral species.

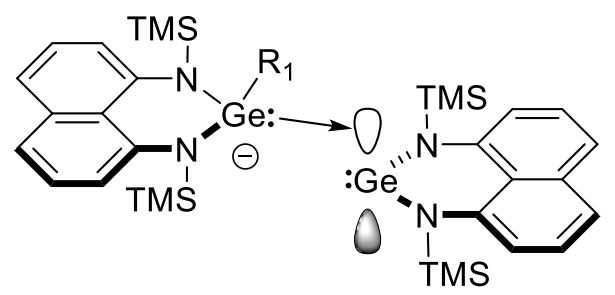

Figure 25: Diagram of potential bonding interaction of 4 and 5.

The different coordination numbers at each of the germanium atoms means that the bite angles of the two $\mathrm{N}$-heterocyclic ligands are different, as can be seen by the $\mathrm{N}(1)-\mathrm{Ge}(1)-\mathrm{N}(2)$ bond angle being $91.70(6)^{\circ}$ while the $\mathrm{N}(3)-\mathrm{Ge}(2)-\mathrm{N}(4)$ bond angle is $92.53(7)^{\circ}$. The angle the germanium sits out of the nitrogennaphthalene plane is also significantly different for the two germanium atoms, with Ge(2) sitting further out of this plane than $\mathrm{Ge}(1)$. The complex appears to be best described as a mixed coordination germanium complex, where the tetrahedral geometry Ge(2) and trigonal pyramidal Ge(1) are in the Ge(II) oxidation state. As can be seen from Table 8 the Ge(2)-N bond lengths of 1.9162(12) and 1.9189(12) A are shorter longer than the $\mathrm{Ge}(1)-\mathrm{N}$ bond lengths of 1.9433(12) and 1.9295(12) $\AA$, and are at the top range of $\mathrm{Ge}(\mathrm{IV})-\mathrm{N}$ bond distances seen in the literature, which tend to range from 1.80-1.91 $\AA$, indicating a Ge(II) oxidation state on $\mathrm{Ge}(2){ }^{73-78}$ The $\mathrm{Ge}(1)$ atom sits significantly out of the NCN plane (the plane defined by the nitrogen atoms and the naphthalene backbone) of the ligand relative to the free germylene unlike the free germylene $\mathbf{3}$, in which the germanium atom sits almost exactly in plane. The $\mathrm{Ge}(1)-\mathrm{N}$ bond lengths are also significantly longer than in the free germylene. Both of these effects are attributed to the donation of electron density into the empty $\mathrm{p}$ orbital of $\mathrm{Ge}(1)$ from $\mathrm{Ge}(2)$, resulting in a change to the idealised $\mathrm{sp}^{2}$ hybridisation present in the free germylene. 


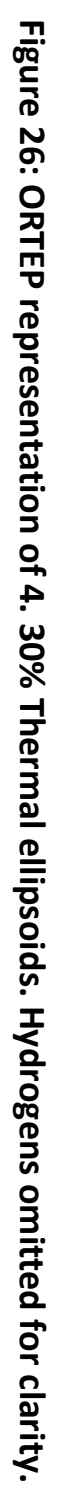
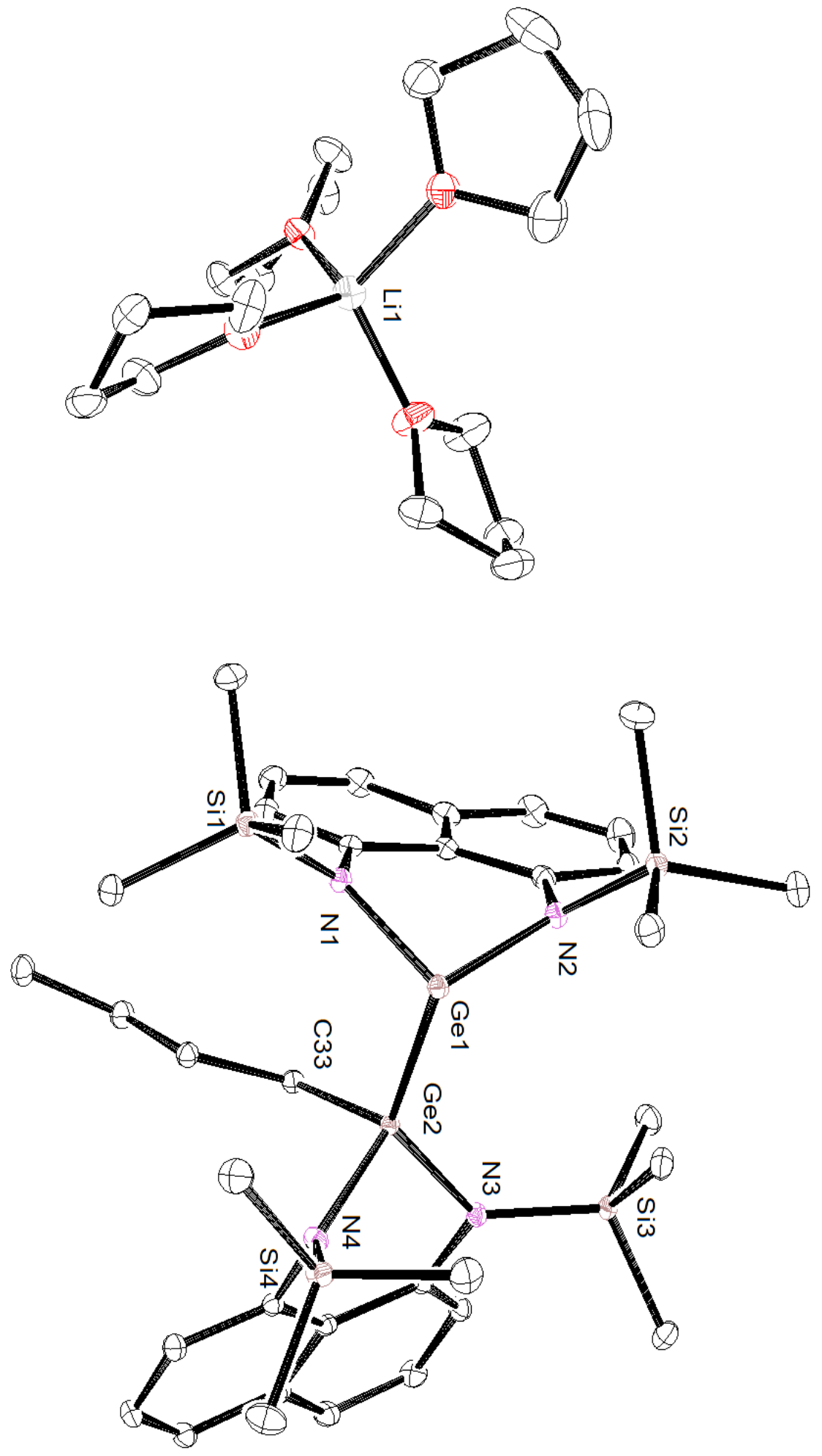
Table 8: Selected bond lengths and angles of 4.

\begin{tabular}{lc}
\multicolumn{2}{c}{ Bond Lengths (̊) } \\
$\mathrm{Ge}(1)-\mathrm{Ge}(2)$ & $2.6222(2)$ \\
$\mathrm{Ge}(1)-\mathrm{N}(1)$ & $1.9433(12)$ \\
$\mathrm{Ge}(1)-\mathrm{N}(2)$ & $1.9295(12)$ \\
$\mathrm{Ge}(2)-\mathrm{N}(3)$ & $1.9162(12)$ \\
$\mathrm{Ge}(2)-\mathrm{N}(4)$ & $1.9189(12)$ \\
$\mathrm{Ge}(2)-\mathrm{C}(33)$ & $1.9901(14)$
\end{tabular}

\begin{tabular}{lc}
\multicolumn{2}{c}{ Bond Angles ( $\left.{ }^{\circ}\right)$} \\
Ge(2)-Ge(1)-N(2) & $102.63(5)$ \\
Ge(2)-Ge(1)-N(1) & $95.32(4)$ \\
Ge(1)-Ge(2)-N(3) & $129.47(4)$ \\
Ge(1)-Ge(2)-N(4) & $107.04(4)$ \\
Ge(1)-Ge(2)-C(33) & $117.93(7)$ \\
N(3)-Ge(2)-C(33) & $102.07(7)$ \\
N(4)-Ge(2)-C(33) & $101.76(6)$ \\
Ge(2)-N(3)-Si(3) & $124.51(8)$ \\
Ge(2)-N(4)-Si(4) & $124.13(7)$ \\
Ge(1)-N(2)-Si(2) & $116.52(6)$ \\
Ge(1)-N(1)-Si(1) & $117.27(6)$ \\
$\mathrm{N}(1)-G e(1)-N(2)$ & $91.70(6)$ \\
$\mathrm{N}(3)-G e(2)-N(4)$ & $92.53(7)$
\end{tabular}

\subsection{2 $\left[\mathrm{Ge}\left(\mathrm{NAPH}^{\mathrm{TMS}}\right) \mathrm{Ge}(\mathrm{Me})\left(\mathrm{NAPH}^{\mathrm{TMS}}\right)\right]^{-}\left[\mathrm{Li}\left(\mathrm{THF}_{4}\right)\right]^{+} \quad 5$}

The ${ }^{1} \mathrm{H}$ NMR spectrum of compound 5 has two new resonances from the TMS environments $(\delta 0.38,0.37$ ppm), which have a small chemical shift downfield from the free germylene $(\delta 0.34 \mathrm{ppm})$. There are two peaks at $\delta 3.11$ and $1.09 \mathrm{ppm}$ integrating for 8 protons each which are assigned to the four THF atoms solvating the lithium ion. These signals are shifted significantly upfield from the free THF in $\mathrm{C}_{6} \mathrm{D}_{6}$ shift, and are also significantly higher field than the solvating THF signals present in the ${ }^{1} \mathrm{H}$ NMR spectrum of 5 , indicating the THF molecules are somehow more shielded in the methyl complex than in the butyl complex 4. The aromatic protons are attributed to 5 signals at $\delta 7.27$ (doublet, $2 \mathrm{H}$ ), 7.22 (doublet, $2 \mathrm{H}$ ), 7.15-7.12 (multiplet, 4H), 6.93 (doublet, 2H) and 6.84 (doublet, $2 \mathrm{H}$ ).

Crystals suitable for an X-ray diffraction study were grown from a concentrated solution of $\mathbf{5}$ in THF. The solid-state data for $\mathbf{5}$ shows that the asymmetric unit contains one molecule of the Ge anion and one THF solvated $\mathrm{Li}^{+}$counterion. The geometry around the germanium atoms is similar to 4 with $\mathrm{Ge}(1)$ having distorted trigonal pyramidal geometry while $\mathrm{Ge}(2)$ has distorted tetrahedral geometry. The $\mathrm{Ge}(1)-\mathrm{Ge}(2)$ bond length of $2.632 \AA$ is longer than is the analogous butyl complex 4 ( $2.622 \AA$ ). The bite angle of the naphthalene based ligands are $91.16^{\circ}$ and $92.68^{\circ}$ for $\mathrm{N}(1)-\mathrm{Ge}(1)-\mathrm{N}(2)$ and $\mathrm{N}(3)-\mathrm{Ge}(2)-\mathrm{N}(4)$ respectively, 
which are similar to the butyl complex $\left(91.70^{\circ}\right.$ and $\left.92.53^{\circ}\right)$, implying that the steric effect of the alkyl fragment has little effect of the bonding of the naphthalene moieties to the germanium atoms. The Ge(1)$\mathrm{N}$ bond distances of 1.954 and $1.947 \AA$ are slightly longer in the methyl complex than the butyl complex (1.943 and $1.930 \AA$ ). The Ge(2)-N bond distances (1.915 and 1.925 $\AA$ ) are slightly shorter than Ge(1)-N bond distance. The trigonal pyramidal geometry at $\mathrm{Ge}(1)$ indicates that the lone pair is stereochemically active, and that therefore the germanium is analogous to 3-coordinate germanium anions $\left[\mathrm{GeR}_{3}\right]^{-}$. Although this anionic germanium is common, it is more frequently seen in cluster compounds or chalcogen and halogen stabilised phosphonium salts. ${ }^{79-84}$ Interestingly, the germanium atom $\mathrm{Ge}(1)$ sits significantly out of the NCCCN plane relative to the free germylene as in 4 . This also aligns with the significant lengthening of the 3 coordinate Ge(1)-N bond lengths from 1.85-1.86 $\AA$ in 3 to $1.94-1.95 \AA$ in 5. This would be expected if $\mathrm{Ge}(2)$ was donating its lone pair of electron density into the empty p orbital of $\mathrm{Ge}(1)$ in the proposed dative bond.

Table 9: Selected bond lengths and angles of 5.

\section{Bond Lengths ( $\AA$ )}

$$
\mathrm{Ge}(1)-\mathrm{Ge}(2)
$$$$
\mathrm{Ge}(1)-\mathrm{N}(1)
$$$$
\mathrm{Ge}(1)-\mathrm{N}(2)
$$$$
\mathrm{Ge}(2)-\mathrm{N}(3)
$$$$
\mathrm{Ge}(2)-\mathrm{N}(4)
$$$$
\mathrm{Ge}(2)-\mathrm{C}(33)
$$

\section{Bond Angles ( $\left.{ }^{\circ}\right)$}

2.6323(6) Ge(2)-Ge(1)-N(2)

1.954(3) Ge(2)-Ge(1)-N(1)

1.947(3) Ge(1)-Ge(2)-N(3)

1.915(3) Ge(1)-Ge(2)-N(4)

1.925(3) Ge(1)-Ge(2)-C(33)

1.980(4) N(3)-Ge(2)-C(33)

$\mathrm{N}(4)-\mathrm{Ge}(2)-\mathrm{C}(33)$

$\mathrm{Ge}(2)-\mathrm{N}(3)-\mathrm{Si}(4)$

$\mathrm{Ge}(2)-\mathrm{N}(4)-\mathrm{Si}(3)$

$\mathrm{Ge}(1)-\mathrm{N}(2)-\mathrm{Si}(2)$

$\mathrm{Ge}(1)-\mathrm{N}(1)-\mathrm{Si}(1)$

$\mathrm{N}(1)-\mathrm{Ge}(1)-\mathrm{N}(2)$

$N(3)-G e(2)-N(4)$
96.30(9)

96.44(9)

115.45(9)

121.09(11)

119.59(11)

101.31(16)

101.97 (16)

121.29(18)

124.97(19)

120.59(17)

118.29(16)

91.16(13)

92.68(14) 


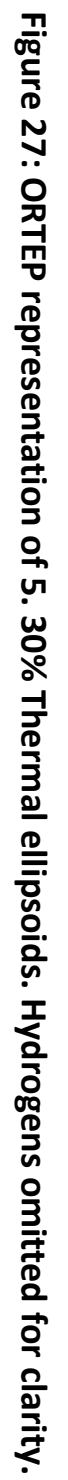

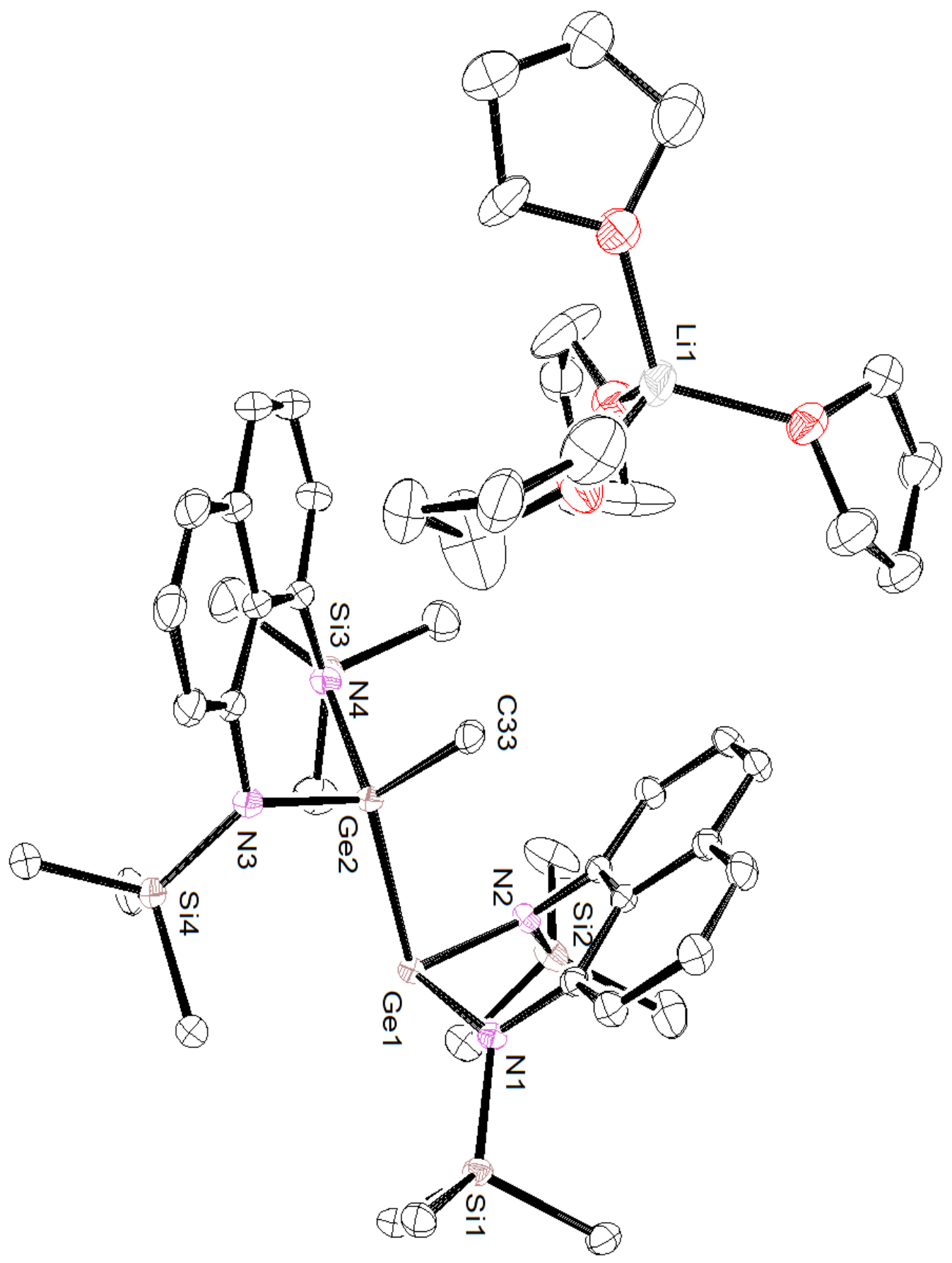


To gain further insight into the bonding of the anionic germanium species DFT calculations were run using the PBE1PBE/def2SVP level of theory for geometry optimisations and PBE1PBE/def2TZVP level of theory for molecular orbital calculations. To simplify the calculations the solvated lithium ion was excluded from calculations and the molecules were instead run with an overall negative charge. For compound 4, the partial charge calculations showed the charge on the two germanium atoms differed, with Ge(1) having a partial charge of $(-0.08)$ while Ge(2) had a positive charge of $(+0.164)$ (appendix 5.5). These values are both lower than the charge on Ge on the free germylene 3, which was calculated on the same basis set to be (+ 0.219). The negative charge of the molecule is primarily located on the carbons of the TMS groups, as well as on the nitrogen atoms in the $N$-heterocyclic ring. The positive charges are primarily located on the silicon atoms of the TMS groups and on Ge(2). The carbons present in the naphthalene rings appear to have both positive and negative partial charges, while the butyl group bonded to Ge(2) has partial negative charges on the carbon atoms, as would be expected of a alkyl fragment.

For comparison to 4, partial charges of the anionic species 5 were also calculated. Once again for simplicity the solvated lithium counterion was omitted from the calculations. After a geometry optimisation the molecular orbitals and partial charges were calculated for the compound as a singlet anion. Comparing the data to the butyl species $\mathbf{4}$, the methyl ligand in $\mathbf{5}$ carries a much higher negative charge $(-0.419$ compared to -0.223 at the $\alpha$-carbon) than the butyl fragment, but the butyl fragment overall carries more of the fraction of relative negative charge compared to the methyl fragment. It can also be seen that $\mathrm{Ge}(2)$ carries a reduced partial positive charge (0.127) relative to $\mathrm{Ge}(2)$ in the butyl complex $4(0.164)$. This reduced charge on $\mathrm{Ge}(2)$ together with the increased charge on $\mathrm{Ge}(1)$ (0.029 compared to -0.008) gives some insight into the electronic state of the Ge complexes, and offers some explanation for why the Ge(1)$\mathrm{Ge}(2)$ bond distance in the methyl complex is longer than in that of the butyl complex (2.632 $\AA$ compared to $2.622 \AA$ ). 
Table 10: Selected bond lengths and angles of 4 and 5, calculated quantities shown in brackets.

Complex $5 \quad$ Complex $4 \quad$ Complex [5] Complex [4]

\begin{tabular}{|c|c|c|c|c|}
\hline $\mathrm{N}-\mathrm{Ge}-\mathrm{N}$ angle $\left({ }^{\circ}\right)$ & 91.16 & 91.7 & [90.17] & [90.29] \\
\hline $\mathbf{N}-\mathrm{Ge}[\mathrm{R}]-\mathbf{N}\left({ }^{\circ}\right)$ & 92.68 & 92.53 & [92.21] & {$[92.30]$} \\
\hline Ge-N (Å) & $1.954,1.947$ & $1.943,1.930$ & [1.936], [1.960] & [1.944], [1.966] \\
\hline Ge[R]-N (Å) & $1.915,1.925$ & $1.916,1.919$ & [1.918], [1.913] & [1.913], [1.917] \\
\hline Ge-R (Å) & 1.9801 & 1.9901 & [1.972] & [1.985] \\
\hline $\mathrm{N}-\mathrm{Ge}(1)-\mathrm{Ge}(2)^{*}$ angle $\left(^{\circ}\right)$ & 99.13 & 102.9 & {$[100.76]$} & {$[100.74]$} \\
\hline N-Ge(2)-Ge(1)* angle $\left(^{\circ}\right)$ & 133.22 & 132.18 & [135.12] & [133.26] \\
\hline Ge-Ge (Å) & 2.6323 & 2.6222 & [2.613] & [2.609] \\
\hline NGeN-NGeN Torsion angle ( $\left(^{\circ}\right)$ & 172.74 & 158.17 & [159.18] & [158.64] \\
\hline
\end{tabular}

Interestingly the NGeN-NGeN torsion angle for the two species is significantly different for the two compounds ( $172.74^{\circ}$ and $158.17^{\circ}$ for the methyl and butyl species respectively). The data would suggest that the steric profile of the butyl substituent plays a much greater role than the methyl group. As shown in Table 10, the computational data agrees well with the the experimental solid-state data, except that there is a descrepancy between the torsion angle for the methyl compound $\mathbf{5}$. The most stable conformation for complexes $\mathbf{4}$ and $\mathbf{5}$ from both the computational optimisation appears to be an eclipsed conformation (as viewed down the Ge-Ge bond) (Figure 28). However, the experimental crystal structure evidence of $\mathbf{5}$ suggests that when the methyl substituent is used the lowest energy conformation is instead an staggered arrangement in which the lone pair on $\mathrm{Ge}(1)$ is staggered between the two $\mathrm{Ge}(2)-\mathrm{N}$ bonds (Figure 28). 


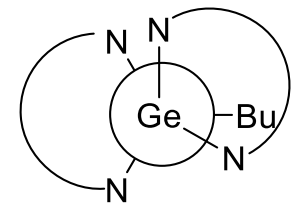

Eclipsed

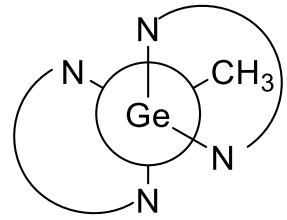

Staggered

Figure 28: Conformations of 4 and 5 as viewed down the $\mathrm{Ge}(1) \mathrm{Ge}(2)$ bond for solid-state data of 4 (eclipsed), and 5 (staggered).

The best description of the bonding present in the compounds $\mathbf{4}$ and $\mathbf{5}$ appears to be an interaction similar to the $\mathrm{Ge}(\mathrm{II})$-> Ge(II) dative bonds of (XXVII) and (XXVIII). ${ }^{47-48}$ Though while XXVII is zwitterionic, 4 and $\mathbf{5}$ instead have a lithium counterion. For XXVII the authors reasoning for the dative germanium-germanium bond was that due to the strong donor capacity of the carbodiphosphorane, making the germanium that the diphosphorane donates its lone pair to is rendered basic enough then react with a second equivalent on $\mathrm{GeCl}_{2}$. The suggested evidence for this is the shortening of the $\mathrm{Ge}-\mathrm{Cl}$ bonds on the 'donor' germanium atom shortening from 2.063 to $1.960 \AA$.

DFT studies conducted on species $\mathbf{4}$ and $\mathbf{5}$ also offer an insight into their structure and bonding. For compound 4 the HOMO has electron density mostly on the Ge(1) lone pair, as well as a smaller electron density on the $\mathrm{Ge}(2)$ lone pair. The HOMO also shows a small amount of electron density present in the $\pi$ system of the naphthalene backbone on Ge(1). The LUMO shows electron density almost exclusively located on the $\pi$ system of the naphthalene ring on the $\mathrm{Ge}(2)$ naphthalene fragment. To gain an indication of the possible reactivity of complex 4 towards small molecules the energy gap of the frontier molecular orbitals was calculated. The HOMO of 4 was stabilised relative to 5 with an energy of $-231.04 \mathrm{~kJ} \mathrm{~mol}^{-1}$. The LUMO was also lower in energy in 4 than in complex 5, with an energy calculated to be $157.53 \mathrm{~kJ} \mathrm{~mol}^{-1}$. This gave a HOMO-LUMO gap of $04.03 \mathrm{eV}$ or $388.57 \mathrm{kJmol}^{-1}$. This data is tabulated in Table 11. This large energy barrier to reactivity was also observed in experimental result, in which stochiometric reactions with a range of unsaturated small molecules (DIC, $\mathrm{PhNCNPh}, \mathrm{PhCOPh}, \mathrm{MesN}_{3}$ ) gave no observable products by ${ }^{1} \mathrm{H}$ NMR spectroscopy. 


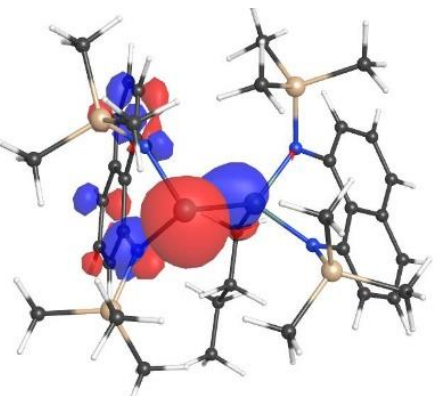

HOMO

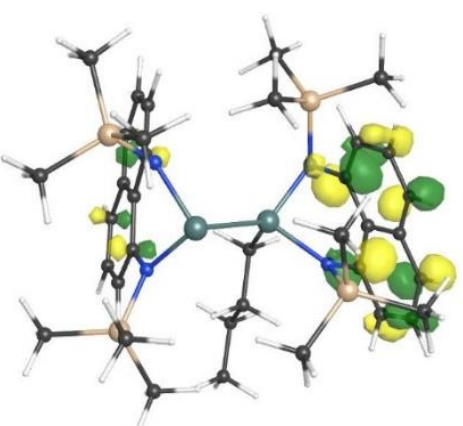

LUMO

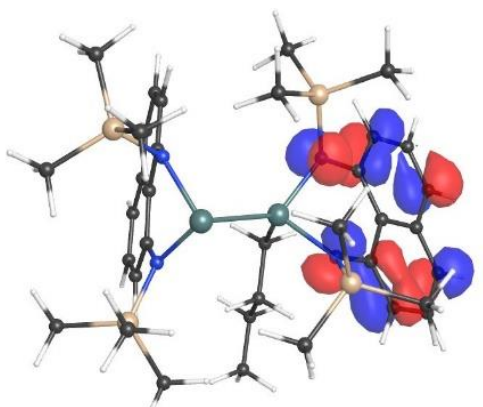

HOMO-1

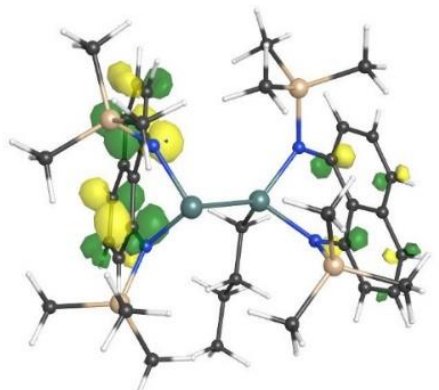

LUMO+1

Figure 29: Frontier molecular orbitals of 4.
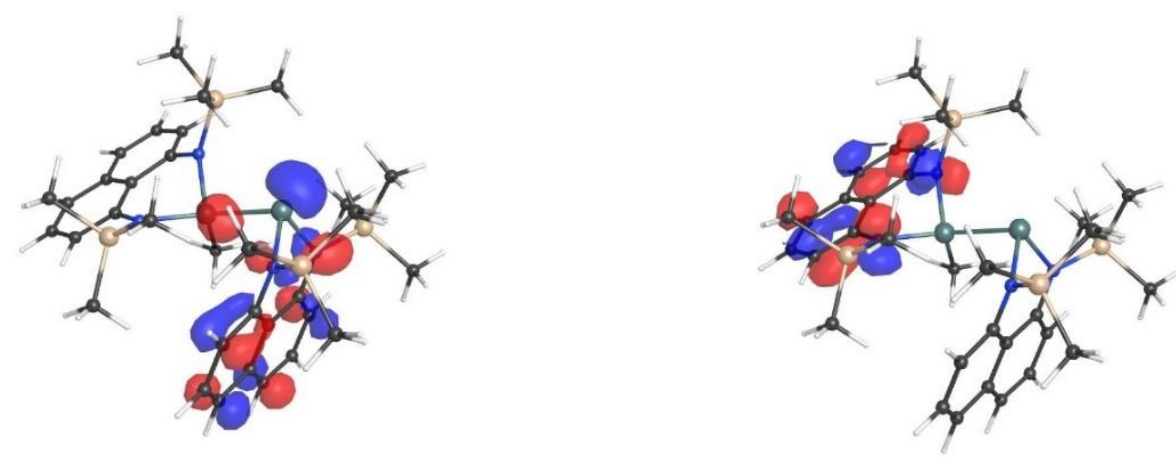

HOMO

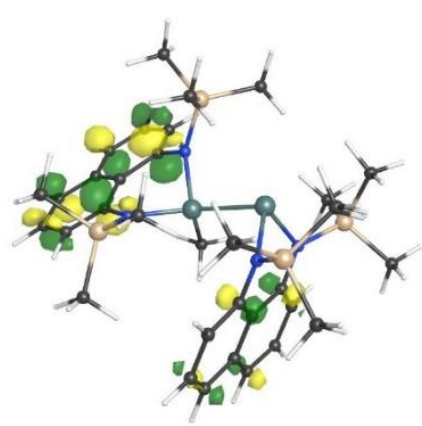

LUMO

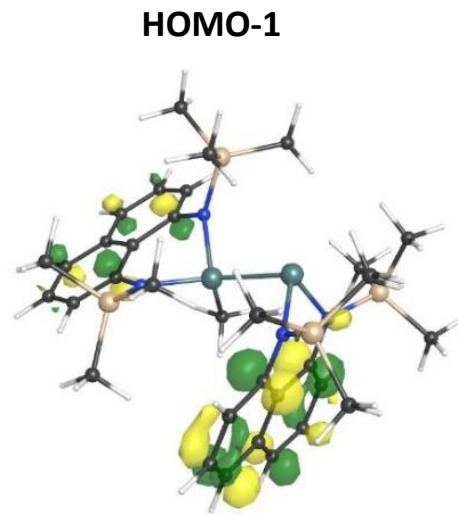

LUMO+1

Figure 30: Frontier molecular orbitals of 5. 
The structure of $\mathbf{5}$ shows that the electron density of the HOMO is primarily a $\sigma$-lone pair orbital with a significant coefficient on the germylene accepter atom and the $\pi$ orbitals of the nitrogen atom bonded to this germanium, with a small amount of electron density on the $\pi$ orbitals of the naphthalene ring of the acceptor fragment to the species. The LUMO electron density is a $\pi$ orbital on the donor germylene naphthalene backbone, with no electron density shown on either of the germanium atoms.

The HOMO-LUMO energy gap was calculated to give an indication of the possible reactivity of the species towards small molecules. The energy of the HOMO was calculated to be $-225.79 \mathrm{~kJ} \mathrm{~mol}^{-1}$ while the LUMO was calculated to be $162.78 \mathrm{~kJ} \mathrm{~mol}^{-1}$. This gives a frontier molecular orbital energy gap of $4.03 \mathrm{eV}$ or 388 $\mathrm{kJ} \mathrm{mol}^{-1}$. This energy gap is relatively large (larger than the HOMO-LUMO gaps of $\mathbf{2}$ and $\mathbf{3}$ ), and may indicate that the germanium species is relatively stable, indeed the physical properties of the species indicated this, with the dimer being stable in the solid state for 6 months and not decomposing when heated in benzene up to $80^{\circ} \mathrm{C}$.

Table 11: Calculated frontier molecular orbital energy gaps for 4 and 5.

\begin{tabular}{|c|c|c|}
\hline & Compound 4 & Compound 5 \\
\hline HOMO-1 (kJ mol-1) & -244.17 & -238.92 \\
\hline HOMO(kJ mol-1) & -231.04 & -225.79 \\
\hline LUMO(kJ mol-1) & 157.53 & 162.78 \\
\hline LUMO+1 (kJ mol-1) & 165.40 & 173.28 \\
\hline HOMO-LUMO (kJ mol-1) & 388.57 & 388.57 \\
\hline Energy (eV) & 4.03 & 4.03 \\
\hline
\end{tabular}




\subsection{Bulky germanium amides.}

\subsubsection{Attempted Synthesis of [Ge(NHAr* $\left.)_{2}\right]$}

Due to the low reactivity of the NHGe molecules $\mathbf{2}$ and $\mathbf{3}$ it was decided that a more reactive germylene species should be synthesised. Bis-amide germylenes have been shown to be more reactive than NHGes, hence it was decided that a new germylene would be synthesised using the bulky primary amine $\left[\mathrm{NH}_{2} \mathrm{Ar}^{*}\right]$ $\left(A r^{*}=2,6\right.$-dibenzhydryl-4-(tert-butyl)phenyl)). The free amine was lithiated using $n$-BuLi in situ before being added to half an equivalent of $\mathrm{GeCl}_{2}$.dioxane (Scheme 18). Although it was expected that this reaction would lead to the bis-amide, the product that was obtained was instead the cyclic amido bridged dimer $6\left[\left(\mathrm{Ge}\left(\mu-\mathrm{NAr}^{*}\right)_{2}\right]\right.$.

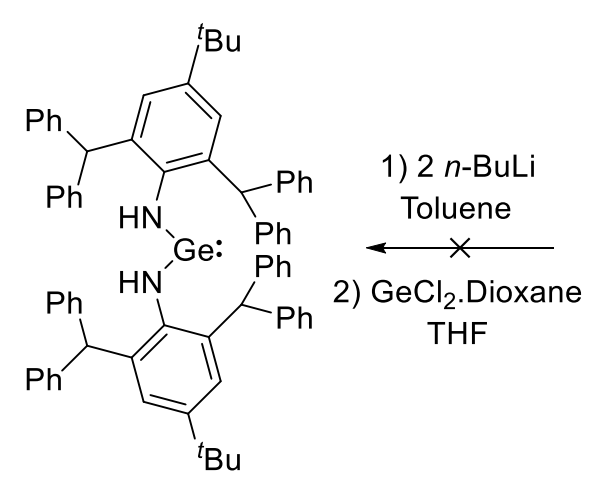

Scheme 18: Reaction of bulky lithiated amines with germanium dichloride.

\subsubsection{Synthesis of $\left[\left(\mathrm{Ge}\left(\mu-\mathrm{NAr}^{*}\right)\right)_{2}\right] 6$}

6 was originally synthesised from the attempt to generate germanium bis-amide $\left[\mathrm{Ge}\left(\mathrm{NHAr}^{*}\right)_{2}\right]$ and was characterized by ${ }^{1} \mathrm{H}$ NMR spectroscopy, ${ }^{13} \mathrm{C}$ NMR spectroscopy, elemental analysis and single crystal X-ray diffraction. The ${ }^{1} \mathrm{H}$ NMR of the crystalline product shows the downfield shift of the tert-butyl ( $\left.{ }^{\mathrm{B}} \mathrm{Bu}\right)$ peak to $\delta 1.13 \mathrm{ppm}$ from $\delta 1.11 \mathrm{ppm}$ (for the free amine) in deuterated benzene. The singlet at $\delta 5.47 \mathrm{ppm}$ from the methine $(\mathrm{CH})$ environment is shifted downfield to $\delta 6.43 \mathrm{ppm}$, which would be expected due to the deshielding effect of the germanium atom. The ${ }^{1} \mathrm{H}$ NMR spectrum also shows a strong new doublet in the aromatic region at $7.27 \mathrm{ppm}$, while the remainder of the aromatic protons are seen as multiplets overlapping with residual toluene in the sample. 
Crystals suitable for an X-ray diffraction study were grown from a concentrated solution of $\mathbf{6}$ in toluene. However, due to incomplete collection of the data resulting in obscurities in the X-ray crystal structure data, analysis of the compound was not possible, however, connectivity was able to be confirmed.

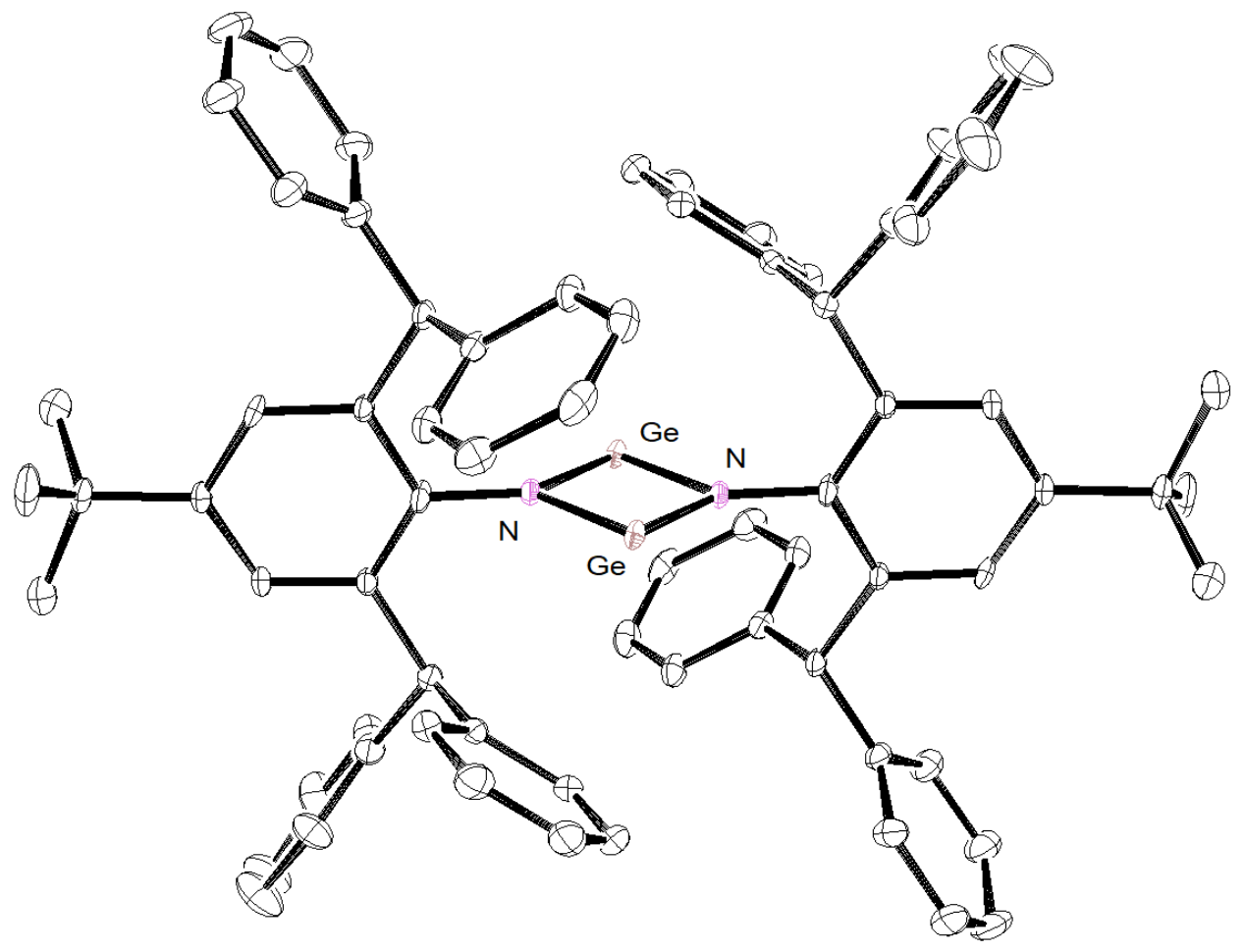

Figure 31: ORTEP representation showing connectivity of 6 . Hydrogens omitted for clarity.

The use of bulky amines such as 2,4,6 tributylaniline, terphenyl ${ }^{\text {Mes }}$ amine and 2,4,6 trifluoroaniline in the synthesis of bulky amide germanium dimers previously been reported, although the manuscripts only offer speculation as to the reaction mechanism for the formation of the dimer. ${ }^{85-87}$<smiles>CC(C)(C)c1cc(C(C)(C)C)c(N2CN(c3c(C(C)(C)C)cc(C(C)(C)C)cc3C(C)(C)C)[GeH2]2)c(C(C)(C)C)c1</smiles>

XLIII<smiles>Cc1cccc(C)c1N1[GeH]N(c2c(C)cccc2C)[GeH2]1</smiles>

XLIV

Figure 32: Amido-bridged germanium compounds XLIII and XLIV.

Three different synthetic pathways were proposed by Lappert et al. in the synthesis of XLIII. Firstly, it was proposed that after the formation of the mono-lithiated amine salt the subsequent addition of 
$\mathrm{GeCl}_{2}$.dioxane could react at the mono-amide chloride bridged dimer XLV(a) (Scheme 19). This would then react further to either go to the bis-amide and onto the imine $\mathbf{X L V}(b)$. Secondly, after formation of the chloride bridged dimer, it was proposed that elimination of $\mathrm{HCl}$ could also generate the germanimine directly $(\mathbf{X L V}(\mathbf{b}))$. Thirdly, the germanium tetracycle could also be formed from the amide bridged complex $\mathbf{X L V}(\mathbf{c})$, with the elimination of an equivalent of free amine. To further investigate the possible reaction pathway, it was decided that we would try to trap Lappert's proposed germanimine intermediate species $\mathbf{X L V}(\mathbf{b})$ with a carbodiimide, to see whether the $[2+2]$ cycloaddition product could be synthesised and characterised.

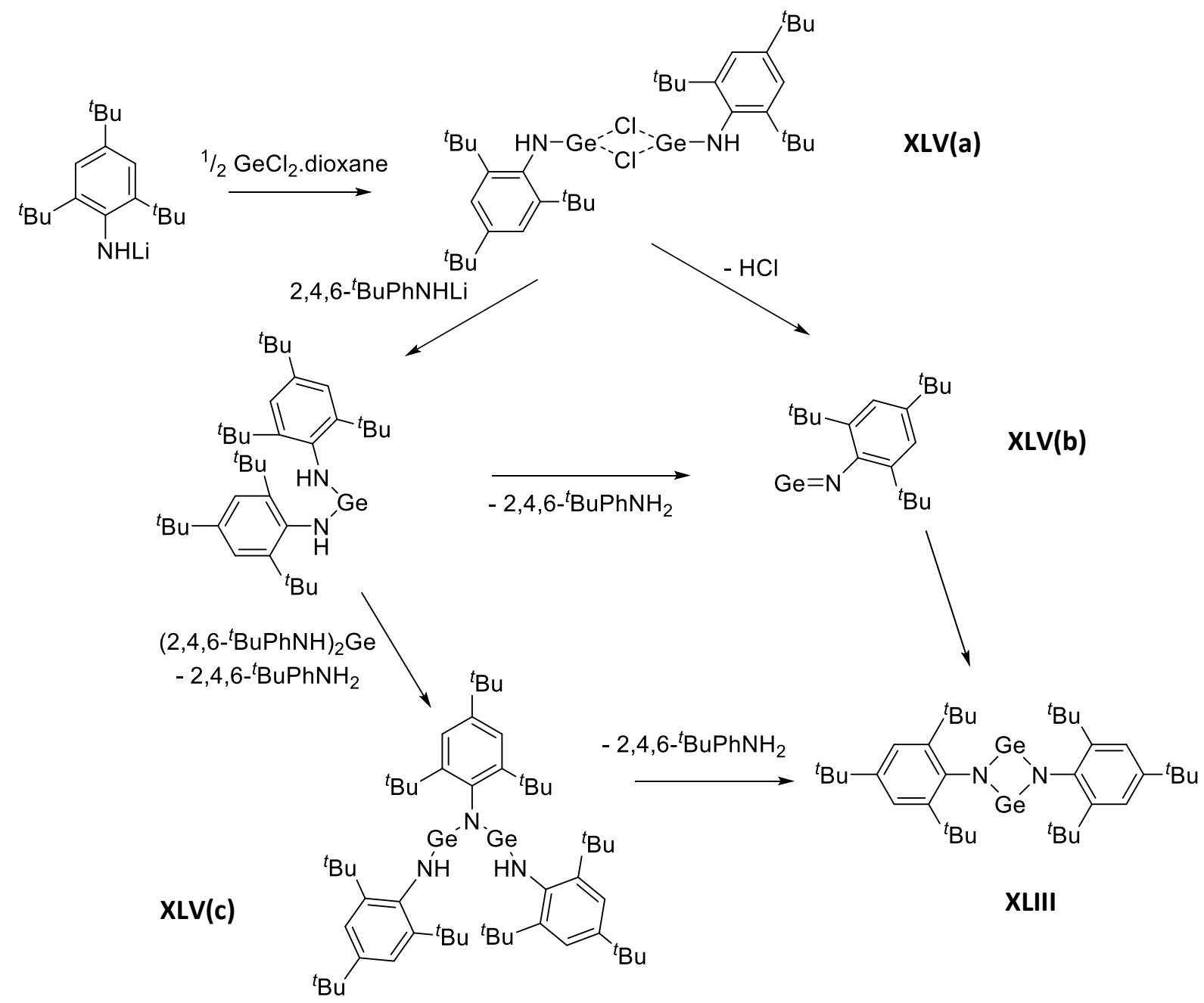

Scheme 19: Lappert's different proposed synthetic pathways to XLIII. 


\subsubsection{Synthesis of $\left[(\mathrm{Cl}) \mathrm{Ge}\left(\mathrm{N}(\mathrm{Cy}) \mathrm{C}(\mathrm{NHCy}) \mathrm{NAr}^{*}\right)\right] \quad 7$}

In order to trap out the possible intermediate germanimine in a [2+2] or [2+3] cycloaddition, the reaction to synthesise 6 was carried out again, but in the presence of dicyclohexylcarbodiimde (DCC). However, instead of the a germylene being synthesised through a cycloaddition with DCC, the product 7 was synthesised in good yields (eq. 16). Compound 7 was synthesised using equal molar amounts of the lithiated amine $\left[\mathrm{NH}_{2} \mathrm{Ar}^{*}\right]$, dicyclohexylcarbodiimide (DCC) and $\mathrm{GeCl}_{2}$.dioxane. After stirring in THF overnight and extraction into toluene the sample was purified by crystallisation at $-30{ }^{\circ} \mathrm{C}$. The crystalline product was characterised by ${ }^{1} \mathrm{H}$ NMR spectroscopy, ${ }^{13} \mathrm{C}$ NMR spectroscopy, elemental analysis and single crystal X-ray diffraction.<smiles>CC(C)(C)c1cc(C(c2ccccc2)c2ccccc2)c(N)c(C(c2ccccc2)c2ccccc2)c1</smiles>

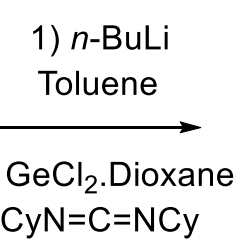

THF

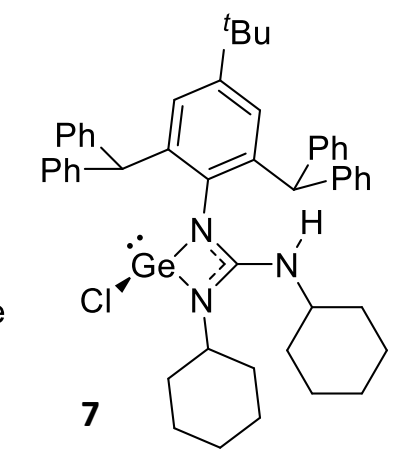

Unfortunately, the synthesis of $\mathbf{7}$ doesn't assist in determining the reaction mechanism for the synthesis of 6 , as the DCC is appears to be reacting with the chloride bridged monoamide germylene similar to $\mathbf{X L V}(\mathbf{a})$, before the generation of the germanimine $\mathbf{X L V}(\mathbf{b})$. The ${ }^{1} \mathrm{H}$ NMR spectrum of complex 7 shows a range of new high field signals from the phenyl rings, though interestingly the compound has more than the 4 expected resonances in this region. Integration of the peaks indicates that while two of the phenyl rings are still equivalent in solution, the other two phenyl rings are in different chemical environments. The ${ }^{1} \mathrm{H}$ NMR spectrum also indicates that the meta protons on the NAr* phenyl backbone are no longer equivalent, which further indicates that the molecule is asymmetric in solution. The tert-butyl chemical shift is upfield from the free amine ( $\delta 1.11 \mathrm{ppm})$ at $\delta 1.08 \mathrm{ppm}$. There are a range of weak signals from the cyclohexyl group from $\delta 1.45$ to 0.10 ppm; however, due to the relatively low intensity of these signals they have been assigned as multiplets since $J_{H H}$ coupling constants could not be determined.

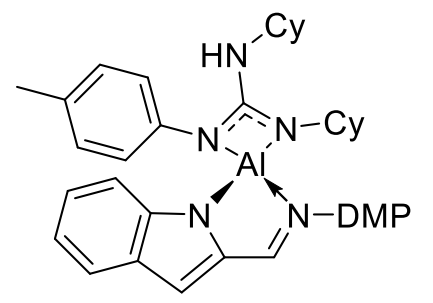

Figure 33:Structure of compound XLVI. 
Crystals suitable for an X-ray diffraction study were grown from a concentrated solution of $\mathbf{7}$ in toluene. The solid-state crystal structure data has one molecule of 7 present in the asymmetric unit, along with two disordered toluene molecules modelled as rigid bodies at half occupancy. The solid -state data reveals that the complex is a germanium-guanidinate compound, with the bulky $\mathrm{N}$-Ar* located $\alpha$ to the germanium atom, with delocalised electron density across the $\mathrm{N}(1)-\mathrm{C}(1)-\mathrm{N}(2)$ atoms. The molecule is trigonal pyramidal at $\mathrm{Ge}$, with the lone pair being stereochemically active. 7 has a $\mathrm{Ge}-\mathrm{Cl}$ bond distance of 2.3148(9) $\AA$, longer than the $\mathrm{Ge}-\mathrm{Cl}$ bond distance of $\mathrm{GeCl}_{2}$. dioxane of $2.250 \AA$. The $\mathrm{Ge}-\mathrm{N}$ bond distances of 7 are 2.024(3) and 1.998(3). The Ar* and cyclohexyl (Cy) group bonded to the $\alpha$ nitrogens are located above and below the Ge(1)-N(1)-N(2) plane. The structure indicates a possible electronic interaction between one of the flanking phenyl rings of the Ar* group and the germanium atom, though it is also possible that this is just a crystal packing effect. The distance of the centroid of this phenyl ring to the germanium atom is $3.3639(15) \AA$. The bite angle of the guanidinate ligand is $65.73(2)^{\circ}$. The most similar compound to $\mathbf{7}$ is the aluminium guanidinate XLVI (Figure 33). ${ }^{88}$ The guanidinate ligand has a bite angle of $67.35^{\circ}$, larger than that of 7 , although this would be expected due to the smaller radii of Al(III) compared to Ge(II). The Al-N bond distances of 2.027 and $1.922 \AA$ are slightly shorter than in 7 , though because the ligand is coordinating to another metal the comparison is not a great one. Other complexes with guaninidate ligands were deemed structurally to different for useful comparisons to be made. ${ }^{89-90}$

Table 12: Selected bond lengths and angles of 7.

Bond Lengths $(\AA)$

Bond Angles ( ${ }^{\circ}$ )

$\begin{array}{ll}\mathrm{Ge}(1)-\mathrm{Cl}(1) & 2.3148(9) \\ \mathrm{Ge}(1)-\mathrm{N}(1) & 2.024(3) \\ \mathrm{Ge}(1)-\mathrm{N}(2) & 1.998(3) \\ \mathrm{C}(1)-\mathrm{N}(3) & 1.349(4) \\ \mathrm{N}(1)-\mathrm{C}(1) & 2.023(3) \\ \mathrm{N}(2)-\mathrm{C}(1) & 1.995(3)\end{array}$

$\begin{array}{ll}\mathrm{Cl}(1)-\mathrm{Ge}(1)-\mathrm{N}(1) & 98.43(8) \\ \mathrm{Cl}(1)-\mathrm{Ge}(1)-\mathrm{N}(2) & 98.03(10) \\ \mathrm{N}(1)-\mathrm{Ge}(1)-\mathrm{N}(2) & 65.73(11) \\ \mathrm{Ge}(1)-\mathrm{N}(1)-\mathrm{C}(1) & 91.75(19) \\ \mathrm{Ge}(1)-\mathrm{N}(2)-\mathrm{C}(1) & 93.33(19) \\ \mathrm{N}(1)-\mathrm{C}(1)-\mathrm{N}(3) & 122.4(3) \\ \mathrm{N}(2)-\mathrm{C}(1)-\mathrm{N}(3) & 128.7(3) \\ \mathrm{Ge}(1)-\mathrm{N}(1)-\mathrm{C}(2) & 136.0(2) \\ \mathrm{Ge}(1)-\mathrm{N}(2)-\mathrm{C}(3) & 135.5(2)\end{array}$




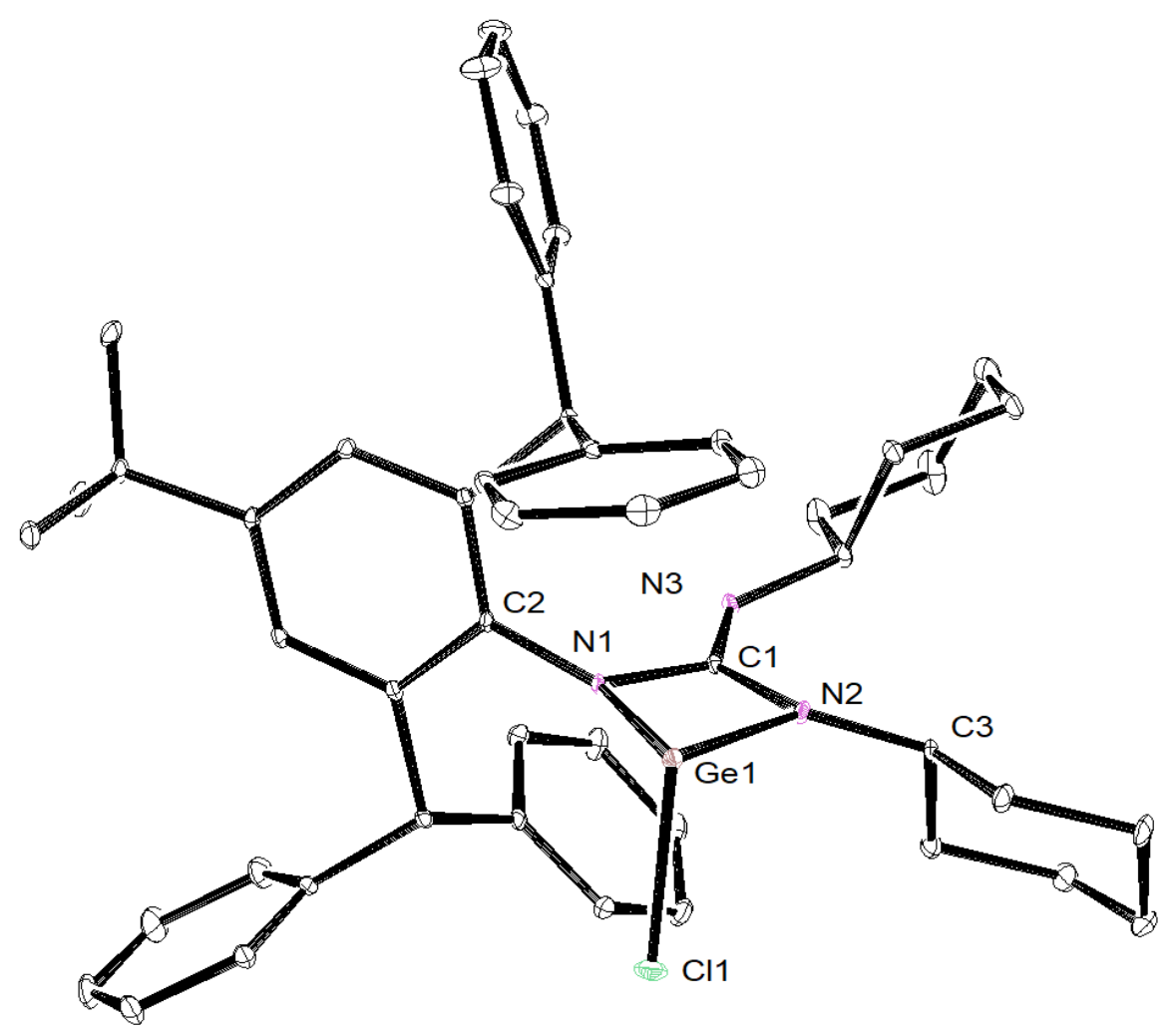

Figure 34: ORTEP representation of 7. 30\% Thermal ellipsoids. Hydrogens omitted for clarity.

\subsubsection{Attempted Synthesis of [Ge(N(Cy)(CNCy)NAr*)]}

To investigate whether 7 could be used as a precursor to the germylene [Ge(N(Cy)(CNCy)NAr*)] the reaction of $\mathbf{7}$ with $n$-BuLi was attempted. The rationale is that the Bu nucleophile could abstract the amido proton on $\mathrm{N}(3)$ of 7 , elimination of $\mathrm{LiCl}$ could result in the dianionic guanidinate ligand on germanium, and a new synthetic route to a range of new germylene compounds would be generated. To a stirred and cooled solution of $\mathbf{7}$ in diethyl ether was added one equivalent of $n$-BuLi. The reaction then had another equivalent of $n$-BuLi added and the solution rapidly went vibrant orange. After workup and purification via recrystallisation a number of lithium salts of the ligand were obtained as crystals. ${ }^{1} \mathrm{H}$ NMR spectroscopy of the reaction mixture identified several resonances for ${ }^{t} \mathrm{Bu}$ environments indicating a range of products that could not be accurately identified. Although the initial equivalent of $n$-BuLi may have deprotonated the $\beta$ carbon of the ligand the likely second equivalent likely generated unstable germanium alkyl species that quickly decomposed to a range of products. 


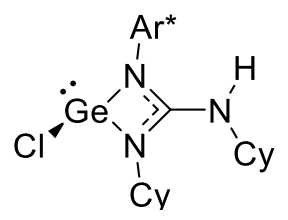<smiles>CCCCC(C)(C)OCC</smiles>

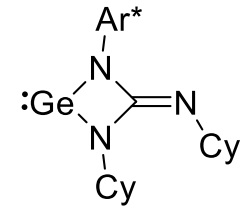

7

\subsection{Synthesis of Germanimines}

One of the synthetic routes proposded to generate zirconium germanium compounds was through the formation of a highly reactive germanimine species $\left(R_{2} G e=N R^{\prime}\right)$ followed by addition of a $\mathrm{Zr}(\mathrm{II})$ species. Germanimines have been synthesised in the literature previously from the oxidative addition of an azide to an $\mathrm{NHGe}$, however, little to no further reactivity studies have been completed and hence the suitability of the species for use in inorganic synthesis remains unknown. ${ }^{41,43,91-92}$

\subsubsection{Attempted Synthesis of [(NAPH $\left.\left.{ }^{\mathrm{TMS}}\right) \mathrm{Ge}(\mathrm{NMes})\right]$}

Our first attempted synthesis of a germanimine was through the treatment of $\mathbf{3}$ with one equivalent of $\left[\mathrm{MesN}_{3}\right]$. The rationale behind using this germylene was its reduced steric bulk on the nitrogen atoms compared to $\mathbf{2}$ and it's relatively easy synthesis. The two compounds were dissolved in deuterated benzene and transferred into an NMR tube fitted with a Young's tap. The reaction was monitored by ${ }^{1} \mathrm{H}$ NMR spectroscopy. No evidence of the germanimine was ever detected, however a new series of resonances indicated the presence of a new product in a 1:1 ratio with 3. Following workup and purification by crystallisation, the product was determined to be the tetrazene 8 .

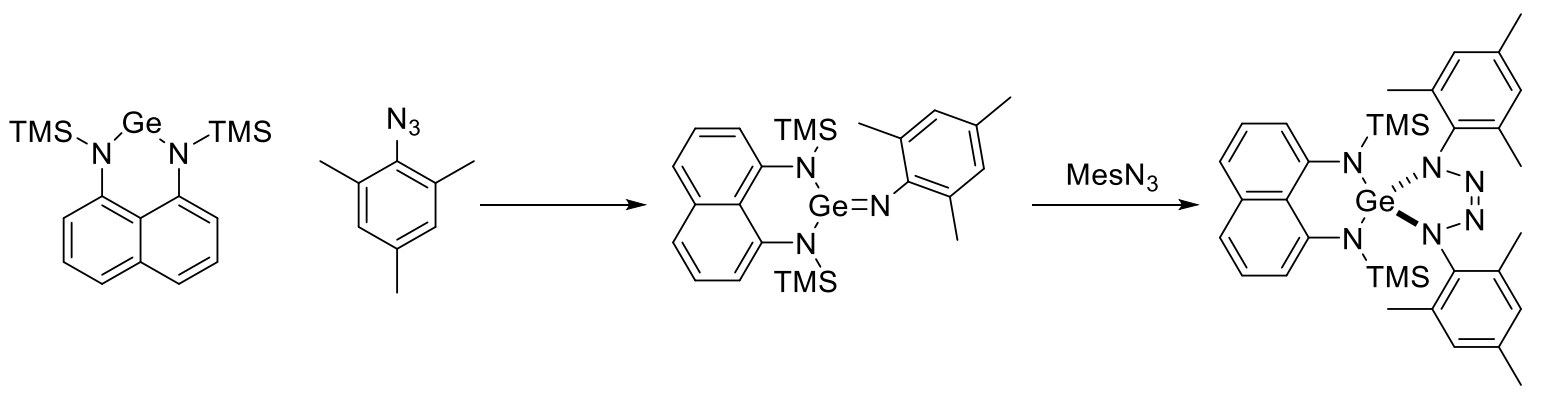

Scheme 20: Synthesis of 8 from the treatment of 3 with [MesN $\left.N_{3}\right]$. 


\subsection{2 $\left[\mathrm{Ge}\left(\mathrm{NAPH}^{\mathrm{TMS}}\right)\left(\mathrm{N}_{4} \mathrm{Mes}_{2}\right)\right]$ Synthesis 8}

Compound $\mathbf{8}$ was synthesised through the addition of two equivalents of mesitylazide to $\mathbf{2}$ at room temperature. The proposed synthetic Scheme for the reaction is shown in Scheme 20. Compound 8 is likely the addition product of the germanimine species with an extra equivalent of azide to give the thermodynamically stable tetrazene product. The reaction appears to happen extremely quickly at RT, as even when the reaction was carried out in the presence of the trapping agent 4-nitrophenylisocyanate the tetrazene 8 was observed as the only product by ${ }^{1} \mathrm{H}$ NMR spectroscopy. The ${ }^{1} \mathrm{H}$ NMR spectrum for 8 has an upfield shift of the TMS resonance to $\delta 0.09 \mathrm{ppm}$, while the two methyl resonances of the azide shift significantly downfield from the free azide to $\delta 2.58$ (ortho methyls) and 2.09 ppm (para methyl) respectively. Four aromatic resonances are also observed in the low field as would be expected for the three naphthalene environments as well as the two meta protons of the phenyl ring of the azide moiety. The integration of these resonances indicates a 2:1 ratio of the mesityl to naphthalene environments.

Crystals suitable for an X-ray diffraction study were grown from a concentrated solution of $\mathbf{8}$ in hexane. The solid-state $X$-ray crystal structure shows one molecule in the asymmetric unit. The structure has a severely distorted tetrahedral geometry at the germanium atom, likely a result of the conformational constraint from the $\left[\mathrm{NAPH}^{\mathrm{TMS}}\right]$ ligand. Like the free germylene the germanium atom lies in plane with the slightly twisted naphthalene ring, with one of each of the coordinating nitrogen atom lying above and below this plane and the TMS groups sitting further above or below the nitrogen atom (in respect of the naphthalene germanium plane). The plane of the mesityl groups lie almost perpendicular to each (the angle between the planes is $104.94^{\circ}$ ) other but are twisted away from the TMS groups presumably to reduce steric interactions. Comparing the structure to the most similar compound in the literature; XXIII the increased steric interaction between the TMS groups and the mesityl groups can be seen. ${ }^{44}$ While in XXIII the phenyl rings sit almost in plane with each other, and this plane is perpendicular to the $\mathrm{N}\left({ }^{\mathrm{t}} \mathrm{Bu}\right)$ Ge- $\mathrm{N}\left({ }^{t} \mathrm{Bu}\right)$ plane, 8 has the $\mathrm{N}(1)-\mathrm{N}(2)=\mathrm{N}(2 \mathrm{i})-\mathrm{N}(1 \mathrm{i})$ plane twisted away from being perpendicular, presumably to minimise the steric interaction between the mesityl groups and the TMS groups on the [NAPH ${ }^{\mathrm{TMS}}$ ] ligand. The Ge(1)-N(1) bond length of $1.846 \AA$ is similar to the Ge-N bond distance in XXIII, as is the $\mathrm{N}(1)-\mathrm{N}(2)$ and $\mathrm{N}(2)-\mathrm{N}(2 \mathrm{i})$ distances of 1.391 and $1.269 \AA$ (1.414 and $1.252 \AA$ resspectively in XXIII). The similar, though more conformationally free tetrazagermole XXIV has similar Ge-N bond lengths for the tetrazene ligand 1.850 and $1.852 \AA$ XXIV also has the $\mathrm{N}-\mathrm{N}$ distances in the tetrazene of 1.394 and 1.360 $\AA$, similar to 8. The Ge(1)-N(3) bond distance of $1.816 \AA$ in $\mathbf{8}$ is longer than that of XXIII (1.808 and 1.812 
$\AA$ ) and shorter than XXIV (1.822 and $1.834 \AA$ A). A summary of the relevant crystallographic bond lengths and distances is summarized in Table 12.

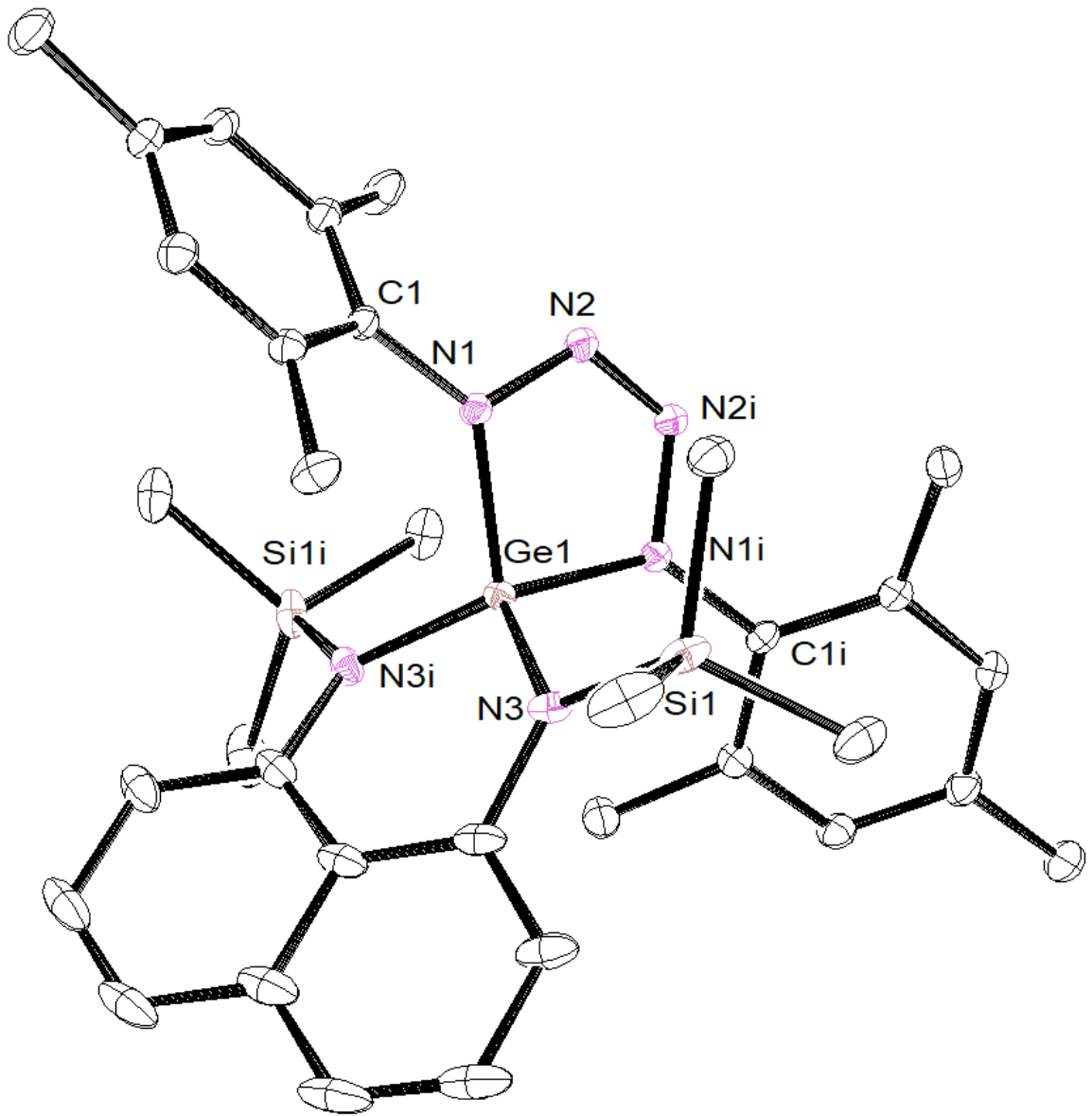

Figure 35: ORTEP representation of $\mathbf{8 . 3 0 \%}$ Thermal ellipsoids. Hydrogens omitted for clarity.

Table 12: Selected bond lengths and angles of 8.

Bond Lengths ( $\mathrm{A})$
$\mathrm{Ge}(1)-\mathrm{N}(1)$
$1.8458(12)$
$\mathrm{N}(1)-\mathrm{C}(1)$
$1.4336(18)$
$\mathrm{N}(1)-\mathrm{N}(2)$
$1.3913(17)$
$N(2)-N(2 i)$
1.269(3)
$\mathrm{Ge}(1)-\mathrm{N}(3)$
1.8155(13)
$N(3)-S i(1)$
1.7789(14)

\section{Bond Angles ( $\left.{ }^{\circ}\right)$}

$\begin{array}{lc}N(3)-G e(1)-N(3 i) & 106.19(9) \\ N(1)-G e(1)-N(1 i) & 84.28(7) \\ N(3)-G e(1)-N(1) & 127.73(5) \\ \text { Ge(1)-N(1)-C(1) } & 132.3(1) \\ \text { Ge(1)-N(3)-Si(1) } & 121.01(7) \\ \text { Ge(1)-N(1)-N(2) } & 112.12(9) \\ N(1)-N(2)-N(2 i) & 115.73(7)\end{array}$




\subsubsection{Synthesis of [(HMDS) $\left.{ }_{2} \mathrm{Ge}(\mathrm{NMes})\right] 9$}

After the synthesis of $\mathbf{8}$ indicated that $\mathbf{3}$ was too reactive to successfully synthesise the desired germanimine it was decided that a similar reaction would be attempted with the germylene $\mathbf{I}$, since it had been shown to be bulky enough to kinetically stabilise other germanimines such as XXI.

Addition of one equivalent of $\left[\mathrm{MesN}_{3}\right]$ to $\mathrm{I}$ in hexane at $-30{ }^{\circ} \mathrm{C}$ resulted in bubbles in the reaction mixture and changed the colour of the solution from orange to pale orange. After concentration of the solution, single, $\mathrm{X}$-ray quality crystals were grown at $-30^{\circ} \mathrm{C}$, which were determined to be the germanimine complex

9.

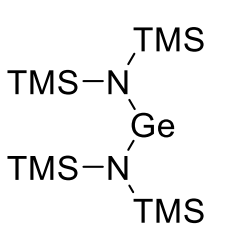

I

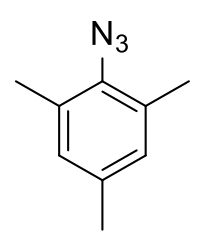

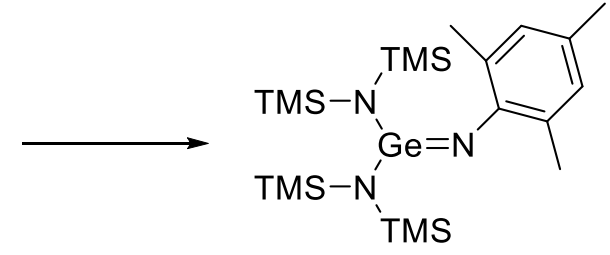

9

The ${ }^{1} \mathrm{H}$ NMR spectrum of 9 shows 4 singlets. There is one peak at $\delta 0.29 \mathrm{ppm}(36 \mathrm{H})$, upfield shifted from the free germylene $(\delta 0.34 \mathrm{ppm})$. The 3 mesityl singlet resonances at $\delta 6.99(2 \mathrm{H}), 2.47(6 \mathrm{H})$ and $2.28(3 \mathrm{H})$ are all downfield shifted from the free azide, as would be expected due to the deshielding of the germylene. The integrals indicate the presence of only one equivalent of azide in the crystals as opposed to two in the germanium-tetrazene compound 8.

Crystals suitable for an X-ray diffraction study were grown from a concentrated solution of $\mathbf{9}$ in hexane. The solid-state X-ray crystal structure of $\mathbf{9}$ is consistent with the presence of a germanium nitrogen double bond. The crystal packs with two molecules asymmetric unit, though as the compounds have similar bond lengths and angles only one molecule will be discussed here. Compound 9 has a distorted trigonal planar geometry around $\mathrm{Ge}(1)$, with the $\mathrm{N}(1)$ atom sitting $9.25^{\circ}$ above the $\mathrm{N}-\mathrm{Ge}-\mathrm{N}$ plane of the HMDS ligands $(\Sigma$ internal angles in 359.91 around the $\mathrm{Ge}(1)$ atom). This trigonal planar arrangement is strong evidence for the $\mathrm{Ge}(1)-\mathrm{N}(1)$ double bond. The $\mathrm{Ge}(1)-\mathrm{N}(1)$ bond length of 1.713(3) $\AA$ is slightly longer than the known germanium nitrogen double bonds in the literature compounds XIX (1.703 $\AA$ ), XXI (1.705 $\AA$ ) and XXIII $(1.682 \AA)$, and may indicate the $\mathrm{Ge}(1)-\mathrm{N}(1)$ bond is more labile towards a range of reactive small molecules. ${ }^{40-41,43}$ The length of this bond is surprising, as the relative steric bulk of the ligand scaffold seems to be less for $\mathbf{9}$ than $\mathbf{X X I}$, and the imine steric bulk is larger in $\mathbf{X I X}$ than complex $\mathbf{9}$. The $\mathrm{Ge}(1)-\mathrm{N}(2)$ 
and Ge(1)-N(3) distances are 1.813(3) and 1.826(3) Å respectively, which is short for Ge(IV)-HMDS bond lengths, which usually range between 1.80-1.91 $\AA^{93-97}$ The short length is probably due to a complex range of factors, though $\left[\mathrm{Ge}^{\mathrm{IV}}(\mathrm{HMDS})_{2} \mathrm{X}_{2}\right.$ ] compounds with other strongly $\sigma$-accepting $\alpha$ atoms such as nitrogen or oxygen tend to have shorter Ge-N(HMDS) bond lengths. ${ }^{75,93,98-99}$ The $\mathrm{N}(1)-\mathrm{C}(1)$ bond distance of 1.394(5) ^̊ is slightly shorter than literature germanimines $\mathbf{X X I}$ and $\mathbf{X I X}$ and there are no unexpected or especially surprising bond angles present in the structure.

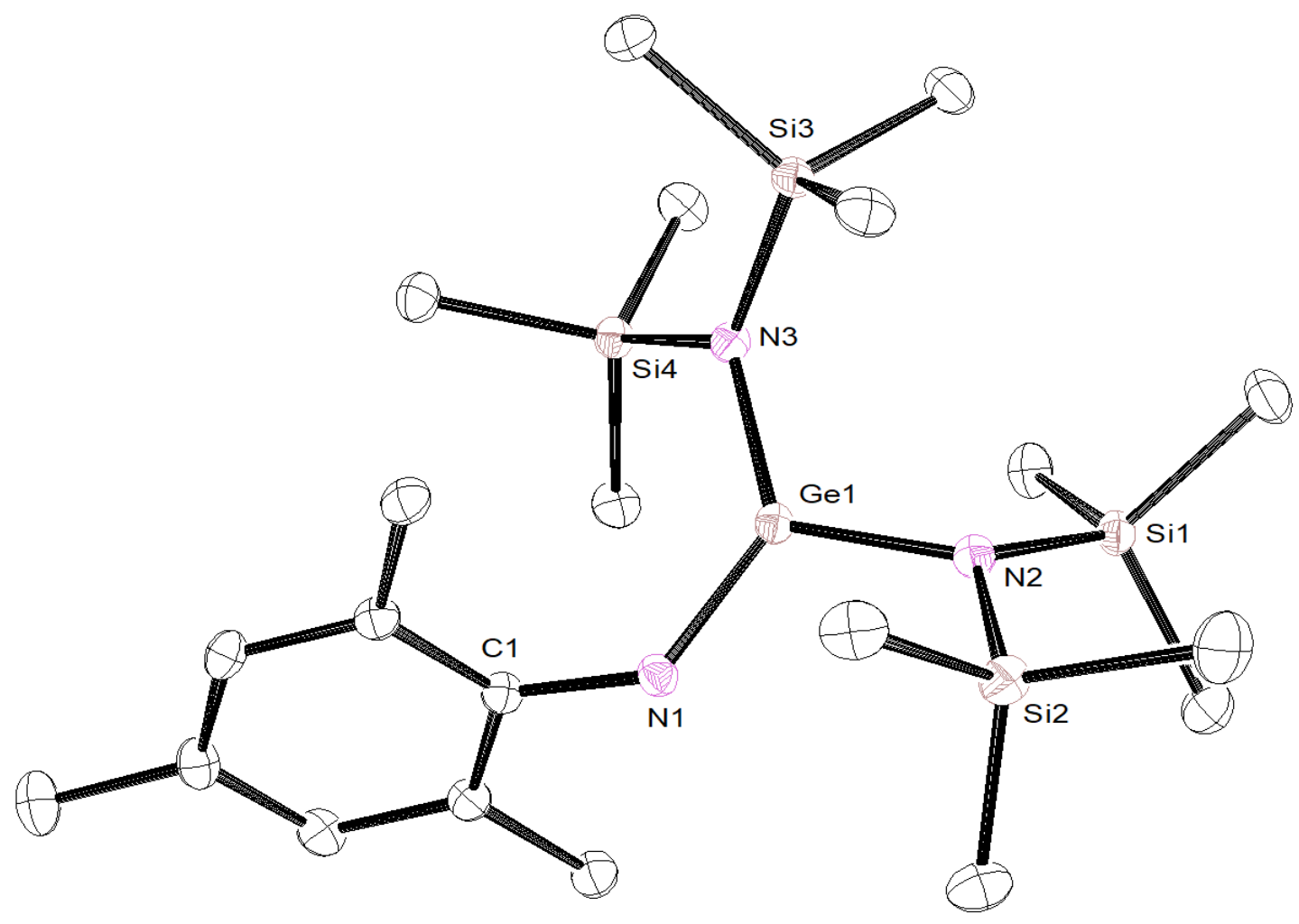

Figure 36: ORTEP representation of 9 (one molecule shown). 30\% Thermal ellipsoids. Hydrogens omitted for clarity. 
Table 13: Selected bond lengths and angles of 9.

\section{Bond Lengths ( $\AA$ )}

$\begin{array}{ll}\mathrm{Ge}(1)-\mathrm{N}(1) & 1.713(3) \\ \mathrm{Ge}(1)-\mathrm{N}(2) & 1.813(3) \\ \mathrm{Ge}(1)-\mathrm{N}(3) & 1.826(3) \\ \mathrm{N}(2)-\mathrm{Si}(1) & 1.754(3) \\ \mathrm{N}(2)-\mathrm{Si}(2) & 1.780(3) \\ \mathrm{N}(3)-\mathrm{Si}(3) & 1.769(3) \\ \mathrm{N}(3)-\mathrm{Si}(4) & 1.764(3) \\ \mathrm{N}(1)-\mathrm{C}(1) & 1.394(5)\end{array}$

\section{Bond Angles ( $\left.{ }^{\circ}\right)$}

$\mathrm{N}(1)-\mathrm{Ge}(1)-\mathrm{N}(2) \quad 112.38(15)$

$\mathrm{N}(1)-\mathrm{Ge}(1)-\mathrm{N}(3) \quad 130.72(14)$

$\mathrm{N}(2)-\mathrm{Ge}(1)-\mathrm{N}(3) \quad 116.81(14)$

$\mathrm{Ge}(1)-\mathrm{N}(2)-\mathrm{C}(1) \quad$ 130.2(3)

$\mathrm{Ge}(1)-\mathrm{N}(2)-\mathrm{Si}(1) \quad 119.54(18)$

$\mathrm{Ge}(1)-\mathrm{N}(2)-\mathrm{Si}(2) \quad 114.99(17)$

Ge(1)-N(3)-Si(3) 117.37(17)

Ge(1)-N(3)-Si(4) 120.57(5)

Si(1)-N(2)-Si(2) 121.56(19)

Si(3)-N(3)-Si(4) 121.75(18)

\subsubsection{Synthesis of [(HMDS $\left.)_{2} \mathrm{Ge}\left(\mathrm{N}_{4} \mathrm{Ph}_{2}\right)\right] 10$}

To investigate the effect of using a less bulky azide for the synthesis of germanimines, I was treated with the less bulky azide $\left[\mathrm{PhN}_{3}\right]$. Addition of 1 equivalent of phenylazide to I at room temperature gave the 1:1 ratio of compound $\mathbf{1 0}$ to $\mathbf{I}$.

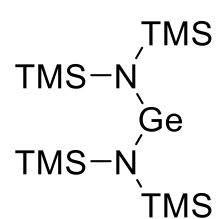

I

'TMS<smiles>Nc1ccccc1</smiles>

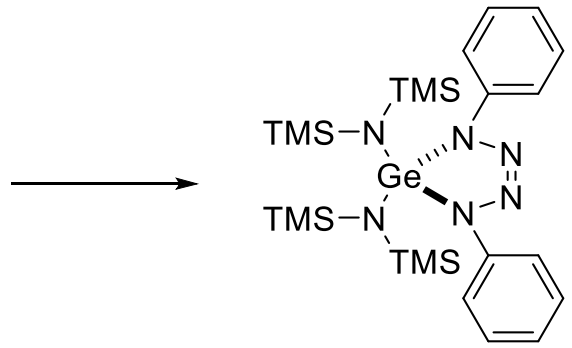

10

The ${ }^{1} \mathrm{H}$ NMR spectrum of the crude reaction mixture indicates that the desired germanimine $\left[(\mathrm{HMDS})_{2} \mathrm{Ge}(=\mathrm{NPh})\right]$ was formed but then undergoes an additional [3+2] cycloaddition with additional azide to from 10.

The ${ }^{1} \mathrm{H}$ NMR spectrum of purified 10 shows 4 resonances. The TMS resonance has shifted upfield to $\delta 0.24$ ppm from $\delta 0.32 \mathrm{ppm}$ in I. The are three resonances for the aromatic protons, a signal at $\delta 7.62 \mathrm{ppm}(2 \mathrm{H})$, a multiplet from $\delta 7.00-6.93 \mathrm{ppm}(4 \mathrm{H})$ and a multiplet from $\delta 6.89-6.80 \mathrm{ppm}(4 \mathrm{H})$. The 2:1 relative integrals 
of the aromatic resonances to the TMS resonance indicate two equivalents of phenylazide has reacted for one equivalent of $\mathbf{I}$.

Crystals suitable for an X-ray diffraction study were grown from a concentrated solution of $\mathbf{1 0}$ in hexane. The solid-state crystal structure data of $\mathbf{1 0}$ has two molecules in the asymmetric unit ( $A$ and $B$ ), however as there two molecules have similar bond lengths and angles only one (A) will be discussed here. There is a distorted tetrahedral geometry around the germanium atoms, with a bite angle of the tetrazene ligand for $\mathrm{N}(1)-\mathrm{Ge}(1)-\mathrm{N}(4)$ of 82.3 (3. Comparing 10 to 8, the HMDS ligands are spaced further apart with a N(5)Ge(1)-N(6) angle of $120.3^{\circ}$, compared to $106.19^{\circ}$ for the N-Ge-N angle of the [NAPH ${ }^{\mathrm{TMS}}$ ] ligand, though this is likely also effected by the constraint of [NAPH ${ }^{\mathrm{TMS}}$ ] being a bidentate ligand. The germanium nitrogen bond distances to the coordinating tetrazene nitrogens are longer in 10 (1.863(7) and 1.877(7) $\AA$ ) than 8 $(1.8458(12) \AA$. The $\mathrm{N}=\mathrm{N}$ bond is $0.026 \AA$ longer in $\mathbf{1 0}$ than $\mathbf{8}$, which also could be due to the decreased steric bulk of the azide or of the ancillary ligand. The intermediate germanimine species was never observed in the ${ }^{1} \mathrm{H}$ NMR in the synthesis of $\mathbf{1 0}$, indicating that the steric bulk of the azide is essential in the synthesis and kinetic stabilisation of germanimines.

Table 14: Selected bond lengths and angles of 10.

\begin{tabular}{lc}
\multicolumn{2}{c}{ Bond Lengths (Å) } \\
Ge(1)-N(1) & $1.875(7)$ \\
$\mathrm{Ge}(1)-\mathrm{N}(4)$ & $1.868(7)$ \\
$\mathrm{N}(2)-\mathrm{N}(3)$ & $1.295(11)$ \\
$\mathrm{Ge}(1)-\mathrm{N}(5)$ & $1.842(6)$ \\
$\mathrm{Ge}(1)-\mathrm{N}(6)$ & $1.831(6)$
\end{tabular}

\section{Bond Angles ( $\left.{ }^{\circ}\right)$}

$\mathrm{N}(1)-\mathrm{Ge}(1)-\mathrm{N}(4)$

$\mathrm{N}(5)-\mathrm{Ge}(1)-\mathrm{N}(6) \quad 120.3(3)$

$\mathrm{N}(1)-\mathrm{Ge}(1)-\mathrm{N}(5) \quad 108.4(3)$

$\mathrm{N}(8)-\mathrm{Ge}(1)-\mathrm{N}(12) \quad$ 109.4(3) 


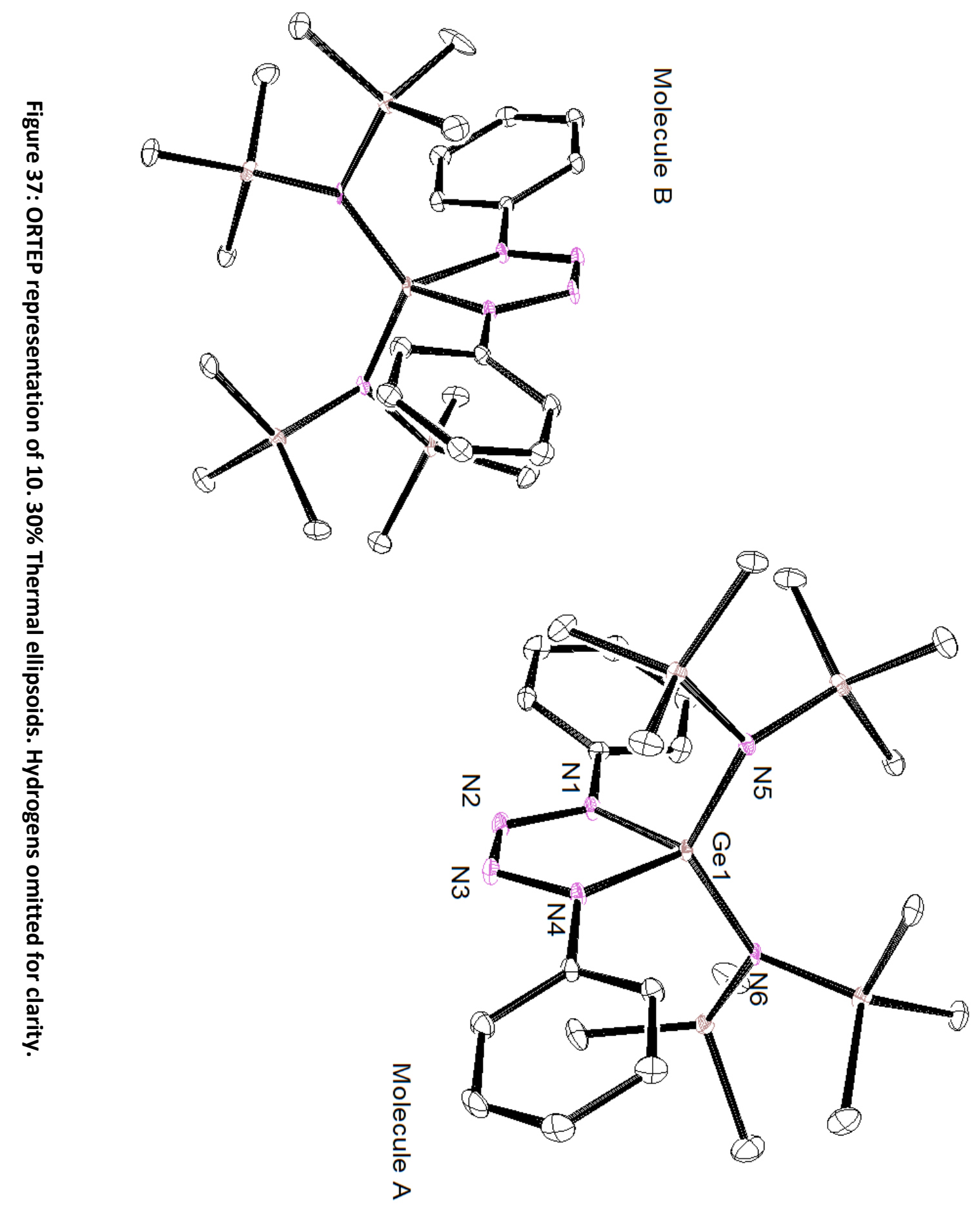




\subsubsection{Synthesis of [(HMDS)Ge(NTMS)(N(TMS)Ar*)] 11}

Complex 11 was synthesised from the reaction of I with the bulky azide $\left[\mathrm{Ar} * \mathrm{~N}_{3}\right](25)$ at $-30{ }^{\circ} \mathrm{C}$ in toluene. The solution initially turned orange and a precipitated out an orange solid as the solution warmed up to room temperature. To crystalise the solid for analysis by X-ray diffraction the suspension was heated to supersaturate the solution, resulting in a yellow solution. The solution was cooled to $-30^{\circ} \mathrm{C}$ to crystalise, resulting in the formation of yellow crystals over 3 days.

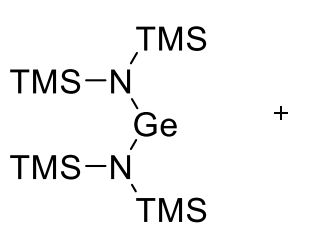<smiles>Nc1c(C(c2ccccc2)c2ccccc2)cc(Br)cc1C(c1ccccc1)c1ccccc1</smiles>

I

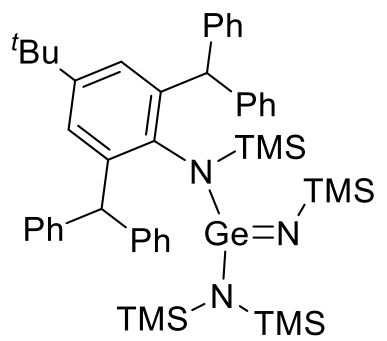

11

An X-ray diffraction study confirmed the synthesis of $\mathbf{1 1}$. This product was presumed to be the rearrangement product of the desired germanimine (Scheme 21). It is likely that the $\mathrm{Ge}=\mathrm{N}$ bond of the desired product would be long, due to the bulk and electronic properties of the Ar* ligand. It would be expected that this bond would be relatively labile due to the long bond length, and as such may readily undergo rearrangement to a more thermodynamic product at elevated temperatures. The easiest way to prove this theory would be to isolate the original germanimine, however, despite several attempts this was not achieved. The desired germanimine could also be trapped out with unsaturated molecules (carbodiimides, acetylene derivatives) to provide evidence that that the species is being formed even if It cannot be isolated.

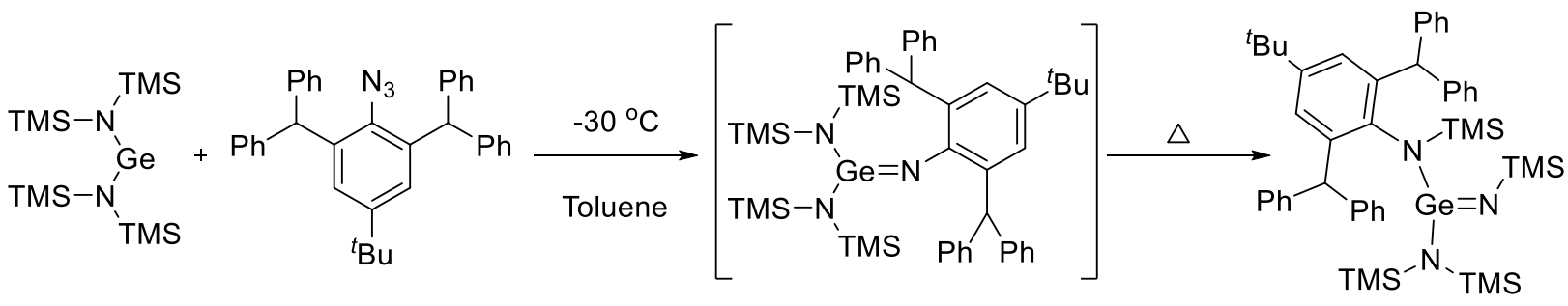

Scheme 21: Synthesis of 11 showing assumed intermediate in brackets. 
The ${ }^{1} \mathrm{H}$ NMR of the crude reaction product identifies three species in solution, assumed to be the desired product [ $\mathrm{HMDS}_{2} \mathrm{GeNAr}^{*}$ ] (identified by the integral ratio 36:9 of the TMS: ${ }^{\text {Bu }}$ resonances), the crystalised product 11 and the impurity from the azide starting material known to be $\mathrm{Ar}^{*} \mathrm{Cl}$ (appendix 5.7). Compound 11 has four high field singlet resonances at $\delta 1.04(9 \mathrm{H}), 0.49(9 \mathrm{H}), 0.45(18 \mathrm{H})$ and $-0.15 \mathrm{ppm}(9 \mathrm{H})$ attributed to the tert-butyl peak of the [N(TMS)Ar*] ligand, the TMS group of the [N(TMS)Ar*] ligand, the [HMDS] ligand and the imido TMS group respectively. The visible aromatic resonances are a doublet at $\delta 7.66 \mathrm{ppm}$ $(4 \mathrm{H})$, a doublet at $7.51 \mathrm{ppm}(4 \mathrm{H})$ and a triplet at $\delta 7.26 \mathrm{ppm}(4 \mathrm{H})$, the other 10 aromatic protons are obstructed by the other signals in the crude NMR. The methine protons have an observable resonance at $\delta 6.34 \mathrm{ppm}(2 \mathrm{H})$, shifted downfield from the free azide ( $\delta 6.06 \mathrm{ppm})$.

The solid-state X-ray crystal structure has one molecule of $\mathbf{1 1}$ and one molecule of toluene present in the asymmetric unit. 11 exhibits a distorted trigonal planar geometry at the germanium atom ( $\Sigma$ angles $=360^{\circ}$ ) with three coordinated nitrogen atoms. The short $\mathrm{Ge}(1)-\mathrm{N}(1)$ bond distance $(1.683(2) \AA ̊ \AA)$ indicates a double bond between $\mathrm{Ge}(1)$ and $\mathrm{N}(1)$. The double bond is significantly shorter than the imido bond in 9 , but falls within the range of known solid state germanium nitrogen double bonds of 1.669-1.863 $\AA^{40-41,43,91-92,100-}$ ${ }^{103}$ The Ge(1)-N(2) bond distance of $1.825(2) \AA ̊$ for $\mathbf{1 1}$ is similar to the $\mathrm{Ge}(1)-\mathrm{N}(2)$ and $\mathrm{Ge}(1)-\mathrm{N}(3)$ distances in 9. 


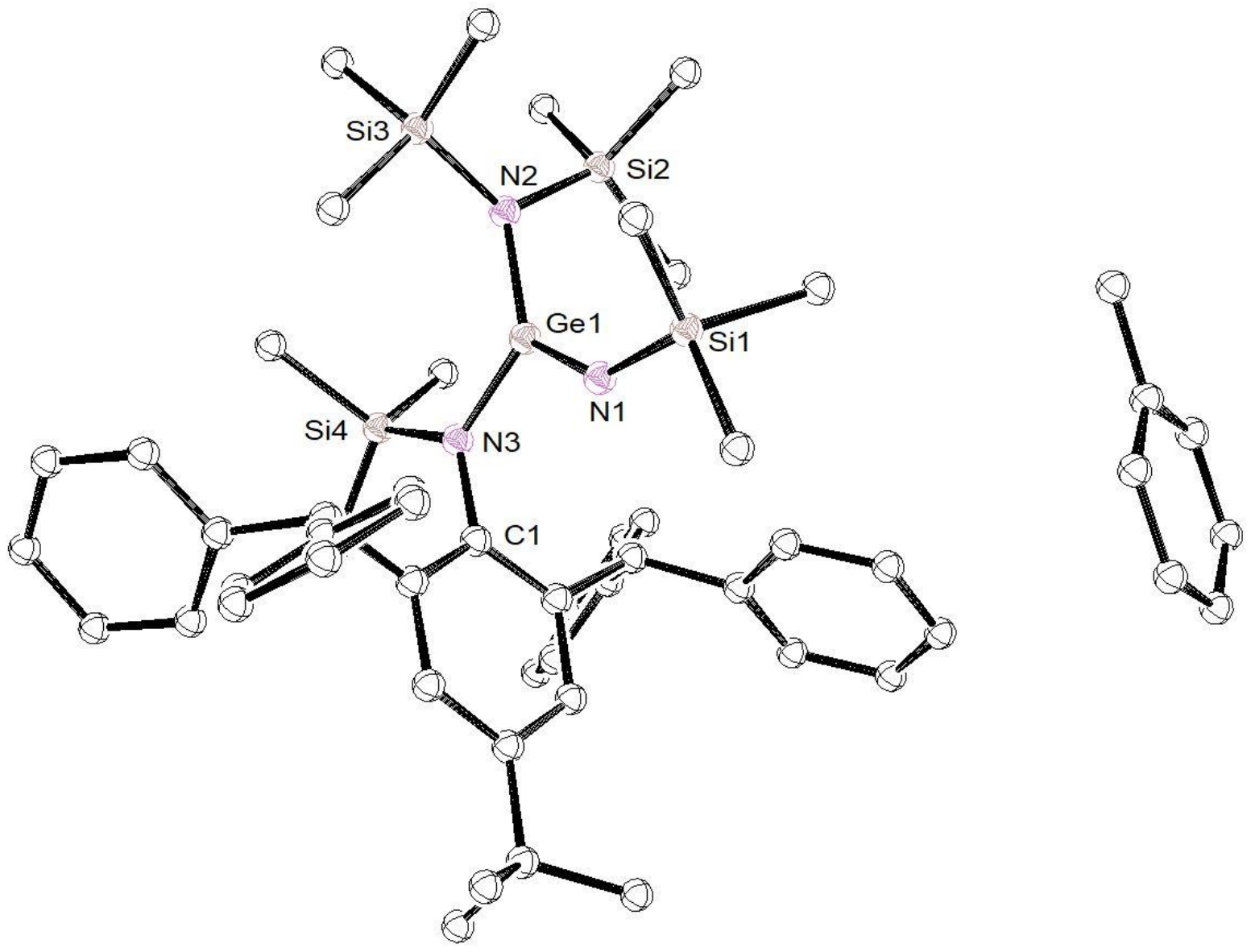

Figure 38: ORTEP representation of $11.30 \%$ Thermal ellipsoids. Hydrogens omitted for clarity.

Table 15: Selected bond lengths and angles of 11.

Bond Lengths (Å)

$\begin{array}{lc}\mathrm{Ge}(1)-\mathrm{N}(1) & 1.683(2) \\ \mathrm{Ge}(1)-\mathrm{N}(2) & 1.825(2) \\ \mathrm{Ge}(1)-\mathrm{N}(3) & 1.815(2) \\ \mathrm{N}(2)-\mathrm{Si}(1) & 1.697(2) \\ \mathrm{N}(2)-\mathrm{Si}(2) & 1.7659(11) \\ \mathrm{N}(2)-\mathrm{Si}(3) & 1.7659(11) \\ \mathrm{N}(3)-\mathrm{Si}(4) & 1.782(2) \\ \mathrm{N}(1)-\mathrm{C}(1) & 1.459(3)\end{array}$

\section{Bond Angles ( $\left.{ }^{\circ}\right)$}

$\begin{array}{cc}N(1)-G e(1)-N(2) & 130.31(10) \\ N(1)-G e(1)-N(3) & 114.0(1) \\ N(2)-G e(1)-N(3) & 115.69(9) \\ \text { Ge(1)-N(3)-C(1) } & 113.37(14) \\ \text { Ge(1)-N(1)-Si(1) } & 141.20(14) \\ \text { Ge(1)-N(2)-Si(2) } & 116.65(6) \\ \text { Ge(1)-N(3)-Si(3) } & 116.65(6) \\ \text { Ge(1)-N(3)-Si(4) } & 127.61(11) \\ \text { Si(2)-N(2)-Si(3) } & 122.81(12)\end{array}$




\subsubsection{Reactivity of [HMDS 2 ) Ge(NMes)], 9 with unsaturated small molecules}

To investigate the reactivity of $\mathbf{9}$, reactions with the unsaturated small molecules phenylactylene and 4nitrophenylisocyanate were carried out. The small sample of molecules tested indicated the germanimine bond was highly reactive towards unsaturated substrates.

\subsubsection{Reactivity towards phenylacetylene}

Treatment of the in situ generated germanimine with 1 equivalent of phenylacetylene led to good yields of the deprotonation product $\left[(\mathrm{HMDS})_{2} \mathrm{Ge}(\mathrm{CCPh})(\mathrm{NHMes})\right], \mathbf{1 1}$, with the phenylacetylene molecule coordinating terminally to the germanium center. The pale-yellow crystals were characterized by ${ }^{1} \mathrm{H} N M R$, ${ }^{13} \mathrm{C} N M R$ and single crystal X-ray diffraction.

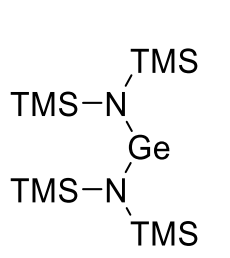

TMS
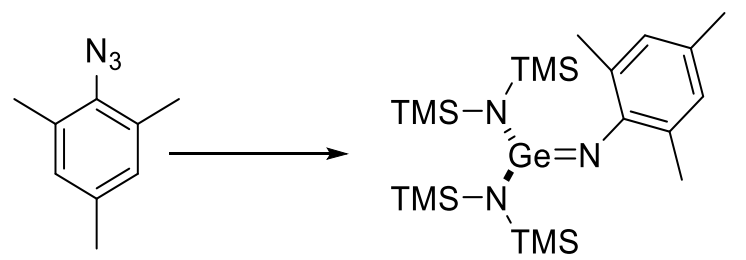

TMS
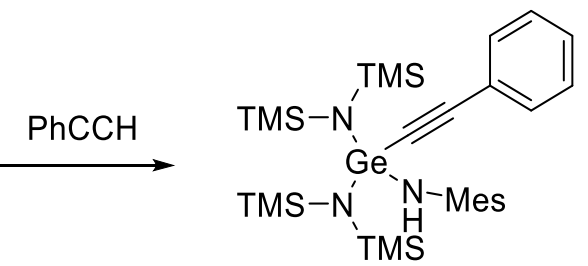

12

Scheme 22: Synthesis of 12 from in situ generation of 9.

The ${ }^{1} \mathrm{H}$ NMR spectrum of 12 shows the downfield shift of the TMS resonance to $\delta 0.47 \mathrm{ppm}$ (from $\delta 0.29$ ppm in 9) indicating more deshielding of the TMS methyl groups. The mesityl resonances are also downfield shifted to $\delta 2.53$ and $2.17 \mathrm{ppm}$ for the ortho and para methyl groups respectively from 9. A new broad peak at $\delta 3.11 \mathrm{ppm}$ that integrates to one proton is attributed to the protonation of the imido nitrogen atom to form an amide. There are four aromatic resonances in the ${ }^{1} \mathrm{H}$ NMR spectrum; a singlet at $\delta 6.82 \mathrm{ppm}$ attributed to the meta protons of the mesityl group are upfield shifted from 9 , in which the resonance was seen at $\delta 6.99 \mathrm{ppm}$. There are three multiplets in the aromatic region $(\delta 7.62,7.43$ and $6.93 \mathrm{ppm}$ ) attributed to the three proton environments of the coordinated phenylacetylene moiety.

Crystals suitable for an X-ray diffraction study were grown from a concentrated hexane solution of $\mathbf{1 2}$. The crystal structure of $\mathbf{1 2}$ shows that there is one molecule in the asymmetric unit. There is a distorted tetrahedral arrangement around the germanium centre. Notable bonding features of compound 12 include the short $\mathrm{C}(22)-\mathrm{C}(23)$ bond distance of $1.199 \AA$ and $\mathrm{Ge}(1)-\mathrm{C}(22)-\mathrm{C}(23)$ bond angle of $174.4^{\circ}$, both of which indicate the presence of a triple bond between $\mathrm{C}(22)$ and $\mathrm{C}(23)$. This bond distance falls within 
the range of known $\mathrm{C} \equiv \mathrm{C}$ bond distances (1.194-1.215 $\AA$ ) in compounds where phenylacetylene terminally coordinates to $\mathrm{Ge}(\mathrm{IV}) .{ }^{104}$ The $\mathrm{Ge}(1)-\mathrm{N}(1)$ bond distance of $1.852 \AA$ is longer than the Ge=N bond distance in complex 9 of $1.713 \AA$, reflecting the decrease in bond order between the germanium atom and the $\mathrm{N}(1)$ atom. The Ge(1)- N(HMDS) bond distances of 1.847 and $1.846 \AA$ are lengthened slightly from 9, plausibly due to the increase in steric bulk around the germanium atom moving from three to four coordinate. The relatively small steric bulk of phenylactylene is reflected in the small $N(3)-G e(1)-N(1), N(1)-G e(1)-C(22)$ and $\mathrm{N}(2)-\mathrm{Ge}(1)-\mathrm{C}(22)$ bond angles of $107.85^{\circ}, 108.10^{\circ}$ and $106.14^{\circ}$. There is a significant difference in the two $\mathrm{N}(1)-\mathrm{Ge}(1)-\mathrm{N}(\mathrm{HMDS})$ bond angles of $103.90^{\circ}$ and $112.79^{\circ}$ for $\mathrm{N}(1)-\mathrm{Ge}(1)-\mathrm{N}(3)$ and $\mathrm{N}(1)-\mathrm{Ge}(1)-\mathrm{N}(2)$ respectively which is likely due to the orientation of the mesityl group towards $N(2)$, while the proton on $\mathrm{N}(1)$ is orientated towards $\mathrm{N}(3)$ when viewed down the Ge(1)-N(1) bond.

Another notable bonding feature of the compound is the migration of the proton from $C(22)$ to $N(1)$, which indicates that the reaction likely proceeds through a deprotonation reaction (Scheme 23). This type of reaction has shown to occur for a variety of main group and transition metal complexes with coordinating nitrogen atoms. ${ }^{105-108}$

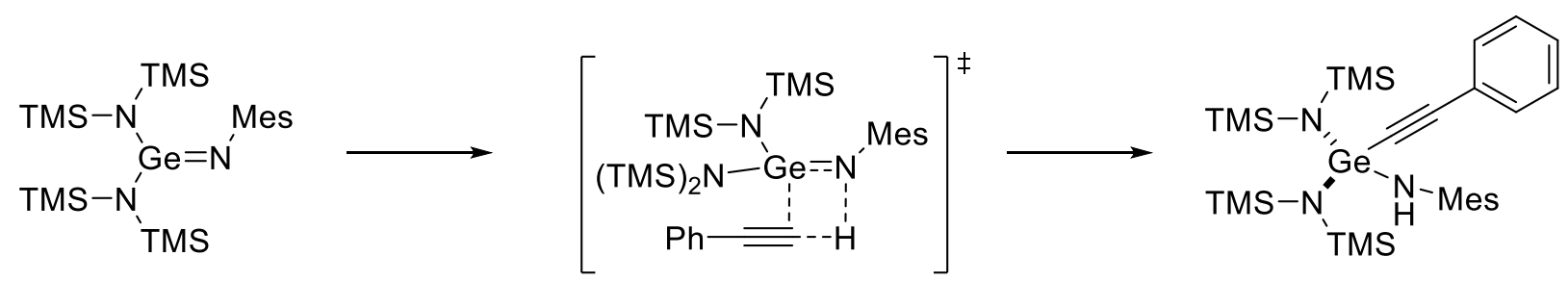

Scheme 23: Proposed reaction pathway for the synthesis of 12 showing a possible transition state. 


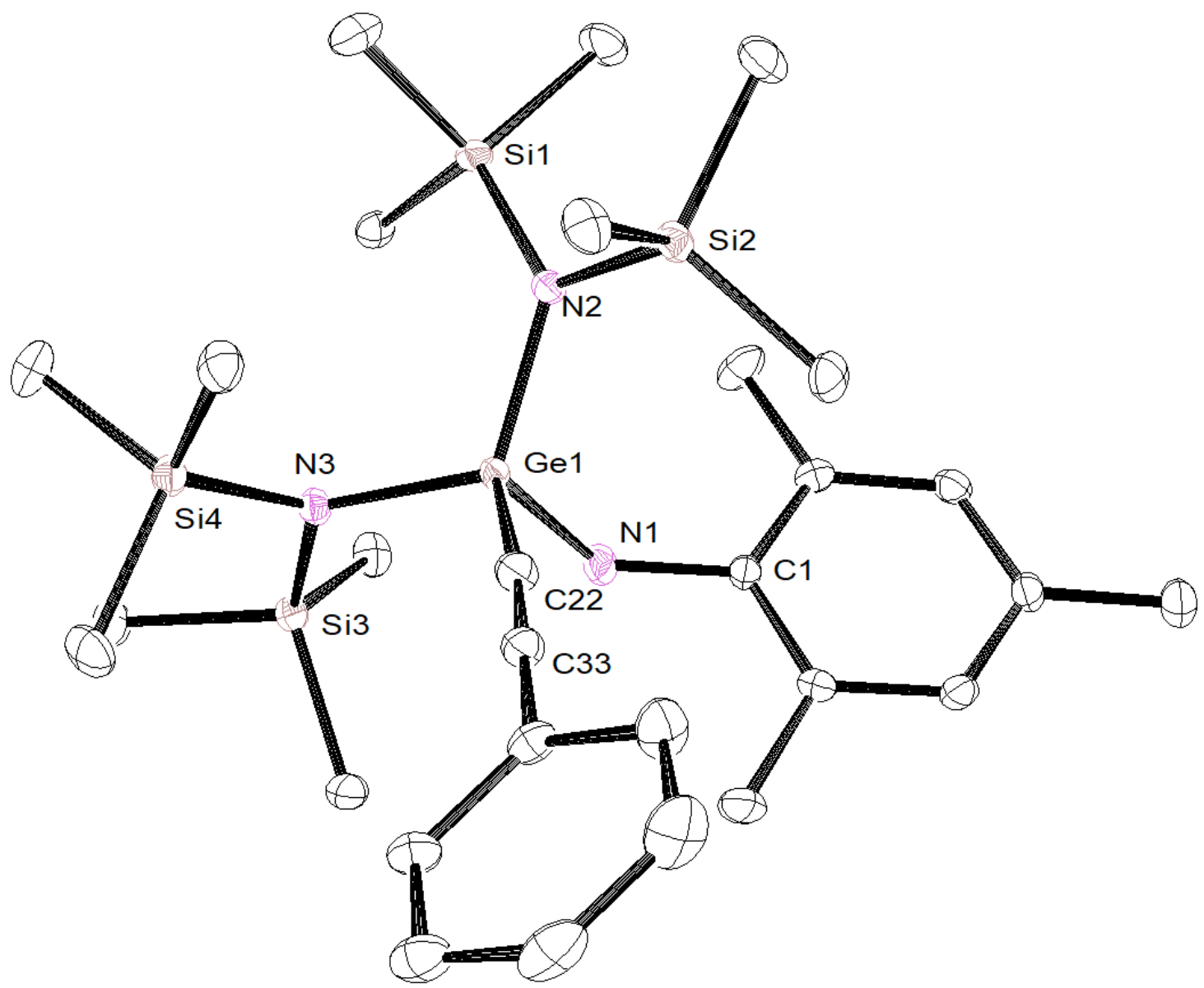

Figure 39: ORTEP representation of $12.30 \%$ Thermal ellipsoids. Hydrogens omitted for clarity.

Table 16: Selected bond lengths and angles of 12.

\begin{tabular}{lc}
\multicolumn{2}{c}{ Bond Lengths (̊̊) } \\
$\mathrm{Ge}(1)-\mathrm{N}(1)$ & $1.8515(19)$ \\
$\mathrm{Ge}(1)-\mathrm{N}(2)$ & $1.8472(17)$ \\
$\mathrm{Ge}(1)-\mathrm{N}(3)$ & $1.8463(17)$ \\
$\mathrm{Ge}(1)-\mathrm{C}(22)$ & $1.917(2)$ \\
$\mathrm{C}(22)-\mathrm{C}(23)$ & $1.199(3)$ \\
$\mathrm{N}(1)-\mathrm{C}(1)$ & $1.427(3)$
\end{tabular}

Bond Angles ( $\left.{ }^{\circ}\right)$
$N(1)-G e(1)-N(2)$
$N(2)-G e(1)-N(3)$
$N(3)-G e(1)-N(1)$
$N(1)-G e(1)-C(22)$
$N(2)-G e(1)-C(22)$
$N(3)-G e(1)-C(22)$
$G e(1)-C(22)-C(23)$
$G e(1)-N(1)-C(1)$

$112.79(8)$

117.52(19)

103.90(8)

107.85(9)

108.10(9)

106.14(9)

$174.4(2)$

$126.16(14)$ 


\subsubsection{Reactivity towards 4-nitrophenylisocyanate}

To investigate if the germanimine $\mathbf{9}$ was reactive towards the isocyanate functional group a NMR scale reaction with 4-nitrophenylisocyanate was carried out. Nitrophenyl isocyanate was chosen over phenyl isocyanate due to the advantages of weighing a solid opposed to a dense liquid. As per the synthesis of 12 the germanimine was generated in situ through the addition of $\left.\left[\mathrm{MesN}_{3} \text { ] to a solution of [Ge(HMDS }\right)_{2}\right]$ in deuterated benzene at room temperature. After 10 minutes a solution of 4-nitrophenylisocyanante was added dropwise and the reaction was transferred to an NMR fitted with a young's tap. The reaction was monitored by ${ }^{1} \mathrm{H}$ NMR spectroscopy. Once the free azide mesityl peaks were no longer observed by ${ }^{1} \mathrm{H}$ NMR spectroscopy was taken back into the glovebox and recrystalised to obtain compound $\mathbf{1 3}$ in a $59 \%$ yield. The crystals were characterized by ${ }^{1} \mathrm{H}$ NMR spectroscopy, ${ }^{13} \mathrm{C}$ NMR spectroscopy and single crystal X-ray diffraction.

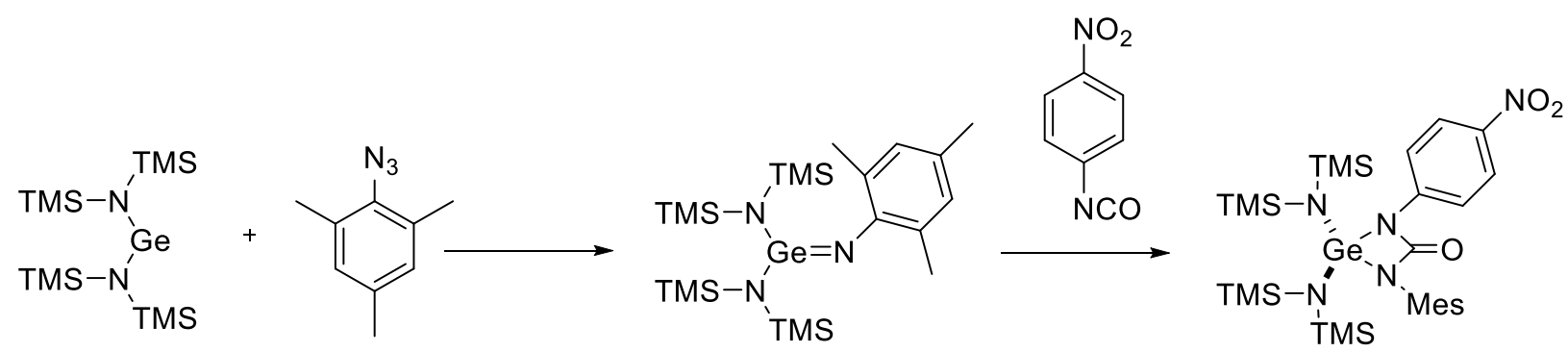

9

Scheme 24: Synthesis of 13 from 9.

The ${ }^{1} \mathrm{H}$ NMR spectrum for 13 shows the upfield shifted TMS resonance $(\delta 0.20 \mathrm{ppm}, 36 \mathrm{H})$ from the germanimine, indicating less deshielding around the TMS groups of the HMDS ligands. The characteristic 2:1 ratio singlet for the methyl groups on the mesityl group are shifted to 2.43 and 2.13 respectively, upfield shifted from the germanimine $9(\delta 2.47$ and $2.28 \mathrm{ppm})$, also indicating reduced deshielding, though this may be expected due to the loss of the extended delocalized system of the mesityl group onto the germanium atom. There are two new low field doublets $(\delta 8.07,7.64 \mathrm{ppm})$, attributed to the two hydrogen environments on the 4-nitrophenyl ring. The extremely electron withdrawing nitro group may account for the significant downfield chemical shift of these two proton environments (relative to phenylisocyanate). ${ }^{109}$

Crystals suitable for an X-ray diffraction study were grown from a concentrated hexane solution of $\mathbf{1 3 .}$ The crystal structure for $\mathbf{1 3}$ confirms the isocyanate has gone to the cycloaddition product opposed to the insertion product or the imido-group exchange as is known for some other elements in higher oxidation 
states. ${ }^{110-111}$ The solid-state crystal structure data shows 2 molecules of $\mathbf{1 3}$ in the asymmetric unit (molecules A and B). However, as these two molecules have extremely similar bond lengths and angles only one molecule will be discussed (molecule A). The geometry around the germanium atom is a distorted tetrahedral, with the $\mathrm{N}(1)-\mathrm{Ge}(1)-\mathrm{N}(2)$ bite angle averaging $70.91^{\circ}$ over the two molecules while the $\mathrm{N}(\mathrm{HMDS})-\mathrm{Ge}-\mathrm{N}(\mathrm{HMDS})$ angle averages $119.13^{\circ}$. The $\mathrm{Ge}(1)-\mathrm{N}(3)$ and $\mathrm{Ge}(1)-\mathrm{N}(4)$ bond lengths of $1.809(2)$ and $1.822(2) \AA$ is over $0.2 \AA$ shorter than is the Ge(IV) complex 12 , possibly due to the increased partial charge on the germanium atom due to the higher number of bonded nitrogen removing more electron density away from the $\mathrm{Ge}(\mathrm{IV})$ atom. The $\mathrm{N}(1)-\mathrm{C}(1)-\left(\mathrm{N}(2)\right.$ angles of $103.2^{\circ}$ is similar to the literature values for compounds with ligands of this type. ${ }^{112} \mathrm{~A}$ literature comparison for complex $\mathbf{1 3}$ is difficult, as the number of 4-coordinate isocyanate cycloaddition products is few, and as far as the author is aware this complex is the first 4-coordinate germanium species with a ligand of this type. 

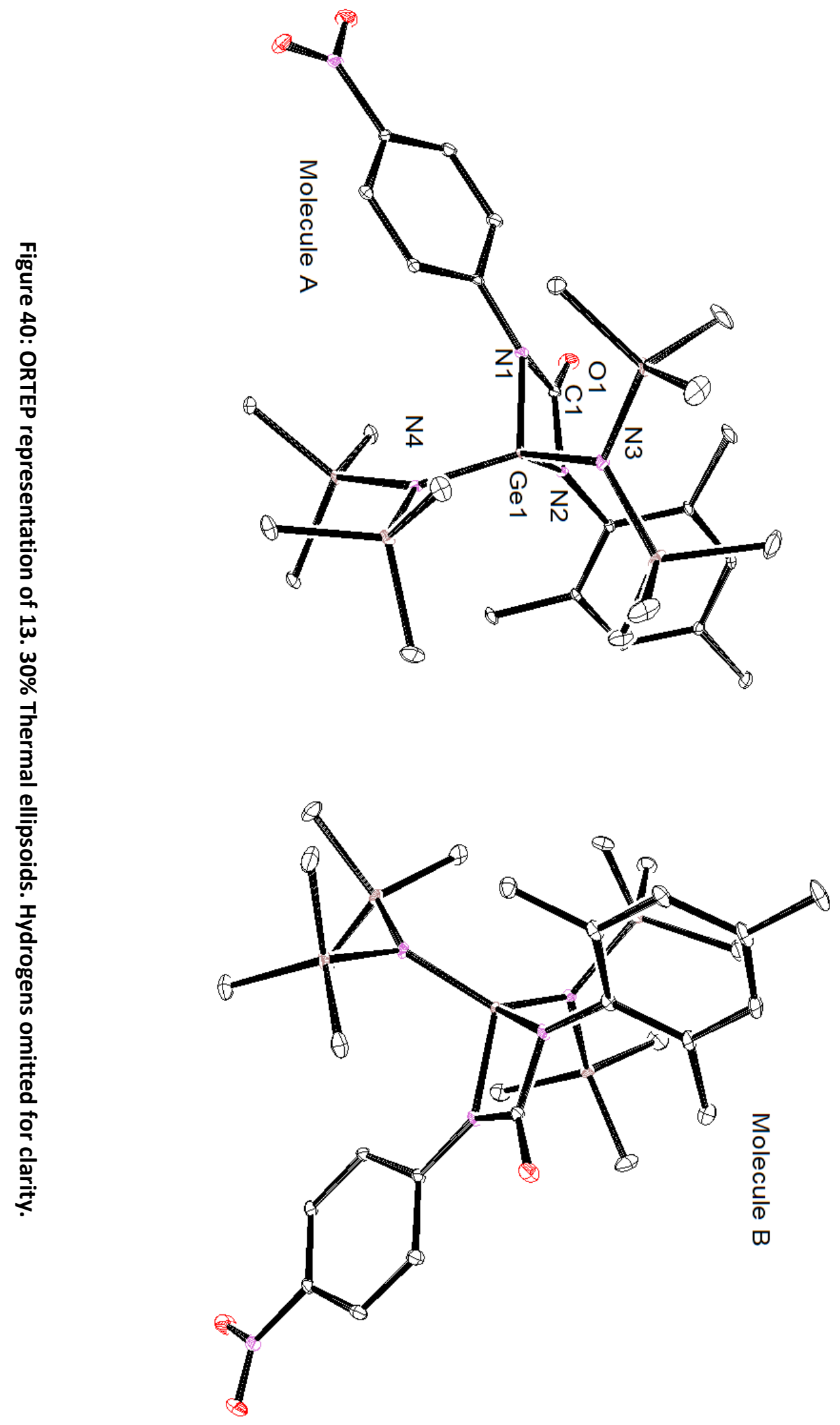
Table 17: Selected bond lengths and angles of 13.

Bond Lengths ( $\AA$ )

$\begin{array}{ll}\text { Ge(1)-N(1) } & 1.894(2) \\ \text { Ge(1)-N(2) } & 1.889(2) \\ \text { Ge(1)-N(3) } & 1.809(2) \\ \text { Ge(1)-N(4) } & 1.822(2) \\ \mathrm{N}(1)-\mathrm{C}(1) & 1.389(4) \\ \mathrm{N}(2)-\mathrm{C}(1) & 1.411(4) \\ \mathrm{C}(1)-\mathrm{O}(1) & 1.215(4)\end{array}$

Bond Angles $\left({ }^{\circ}\right)$

$\begin{array}{cc}\mathrm{N}(1)-\mathrm{Ge}(1)-\mathrm{N}(2) & 70.94(10) \\ \mathrm{N}(3)-\mathrm{Ge}(1)-\mathrm{N}(4) & 118.17(11) \\ \mathrm{N}(1)-\mathrm{C}(1)-\mathrm{N}(2) & 103.2(2) \\ \mathrm{N}(1)-\mathrm{C}(1)-\mathrm{O}(1) & 128.4(3) \\ \mathrm{N}(2)-\mathrm{C}(1)-\mathrm{O}(1) & 128.3(3)\end{array}$

\subsection{Summary of Results}

The novel germylenes $\mathbf{2}$ and $\mathbf{3}$ were synthesised and their reactivity was investigated using MO DFT calculations which indicated that $\mathbf{2}$ would be the more reactive germylene as determined by the calculated HOMO-LUMO energy gap. However, when $\mathbf{2}$ and $\mathbf{3}$ were treated with half an equivalent on $n$-BuLi or MeLi compound $\mathbf{2}$ did not give any reaction products, while compound $\mathbf{3}$ gave the donor-acceptor complexes $\mathbf{4}$ and 5. This indicated that although germylene $\mathbf{2}$ was theoretically the more reactive germylene the role of steric bulk at the $N$ positions of the ligands may have further influenced the germylenes reactivity. When germylene $\mathbf{3}$ was treated with an azide in the attempted synthesis of a germanimine, the tetrazene product $\mathbf{8}$ was instead formed, indicating that at room temperature the germanimine formed in situ is more reactive than towards additional azide than 3. The germanimine 9 was successfully synthesised from the treatment of $\left[\mathrm{MesN}_{3}\right]$ to I, however when I was treated with the less bulky azide $\left[\mathrm{PhN}_{3}\right]$ the tetrazene product $\mathbf{1 0}$ was formed under the same conditions, indicating the importance of the steric bulk of the azide. The reactivity of 9 with phenylacetylene and 4-nitrophenylisocyanate to form 12 and 13 showed the $\mathrm{Ge}=\mathrm{N}$ bond was reactive towards unsaturated small molecules. The treatment of I with the more bulky azide $\left[\mathrm{Ar}^{*} \mathrm{~N}_{3}\right]$ gave a range of products, however, the germanimine $\mathbf{1 1}$ was isolated and characterised. $\mathbf{1 1}$ was presumed to be the thermal rearrangement of the germanimine $\left[(\mathrm{HMDS})_{2} \mathrm{Ge}\left(\mathrm{NAr}^{*}\right)\right]$. 


\section{Results and Discussion: Group 4 Chemistry}

\subsection{Synthesis of Amides and Imidozirconocene species}

\subsubsection{Synthetic Strategy}

The research in this chapter investigates further the work of Bergman et al. and the use of thermolysis reactions to synthesise imidozirconocenes from cyclopentadienyl supported zirconium amides. ${ }^{49,51,54,113-}$ 114 (equation 21). These amidozirconocenes and imidozirconocenes were synthesised for their use in the synthesis of 1 (Scheme 1).
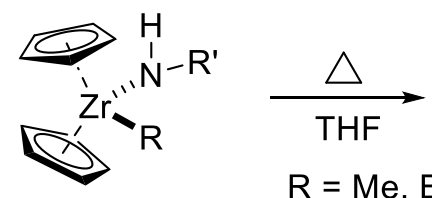

$\mathrm{R}=\mathrm{Me}, \mathrm{Bn}$

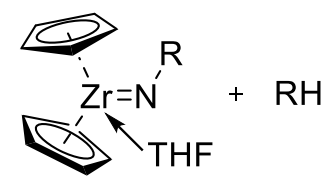

$\mathrm{R}^{\prime}=\mathrm{Ph},{ }^{\mathrm{t}} \mathrm{Bu}, \mathrm{Ar}{ }^{*}, \mathrm{Dipp}, \mathrm{DMP}$

Although the literature syntheses generate the zirconium amides in situ, in this project the amides were isolated and characterised where possible to gain insight into their structure and bonding. The amides were synthesised in two different methods. The addition of a free amine to $\left[\mathrm{Cp}_{2} \mathrm{ZrMe}_{2}\right]$ or $\left[\mathrm{Cp}_{2} \mathrm{ZrBn}_{2}\right]$ at $100{ }^{\circ} \mathrm{C}$ was used for the successful synthesis of compounds 17,18 and 19 while 14, 15 and 16 were synthesised from the salt metathesis of $\left[C p_{2} \operatorname{Zr}(\mathrm{Me})(\mathrm{Cl})\right]$ with the a lithiated amine. Two different alkyl groups ( $\mathrm{Me}=\mathrm{CH}_{3}, \mathrm{Bn}=\mathrm{CH}_{2} \mathrm{Ph}$ ) were used due to availability of starting materials, reactivity and atom efficiency. Although the salt metathesis synthetic route was the only successful method to an isolated imidozirconocene (19), this route was avoided due to the low overall yields $(5.6-20.5 \%)$ due to the high number of reactions and resulting low atom efficiency required to synthesise imidozirconocenes relative to other pathways (Scheme 25).

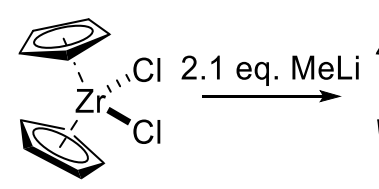

$50-80 \%$

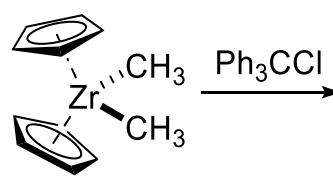

$70-80 \%$

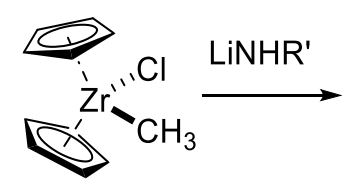

$32-64 \%$<smiles>[R]N[C@]1(C)C=C[C@H]1C1(C)CC1</smiles>

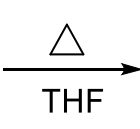

$>50 \%$

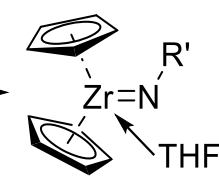

THF

Scheme 25: Synthetic route to imidozirconocenes via salt methathesis. 
Although it has been reported that the species $\left[\mathrm{Cp}_{2} \mathrm{Zr}(\mathrm{Me})(\mathrm{Cl})\right]$ could be synthesised through the addition of 1 equivalent of MeLi at $-30{ }^{\circ} \mathrm{C}$, it was found that the major product under these conditions was actually the bis-methyl species $\left[\mathrm{Cp}_{2} \mathrm{ZrMe} \mathrm{H}_{2}\right]$. As such an alternative literature procedure was used to synthesise $\left[C p_{2} \operatorname{Zr}(\mathrm{Me})(\mathrm{Cl})\right]$ using trityl chloride in ether to give the monochloride species and triphenylethane, with the two products being easily isolated due to the relative insolubility of $\left[\mathrm{Cp}_{2} \mathrm{Zr}(\mathrm{Me})(\mathrm{Cl})\right]$ in hexane.

\subsubsection{Synthesis of $\left[\mathrm{Cp}_{2} \mathrm{Zr}\right.$ (NHDipp)(Me)] 14}

The reaction of $\left[\mathrm{Cp}_{2} \mathrm{Zr}(\mathrm{Me})(\mathrm{Cl})\right]$ with one equivalent of the mono-lithiated amine; [LiNHDipp] in diethyl ether resulted in the formation of the amide $[\mathrm{Cp} 2 \mathrm{Zr}$ (NHDipp)(Me)], 14 in a moderate yield (64\%). The compound was characterized by ${ }^{1} \mathrm{H}$ NMR spectroscopy, ${ }^{13} \mathrm{C}$ NMR spectroscopy and by single crystal X-ray diffraction.

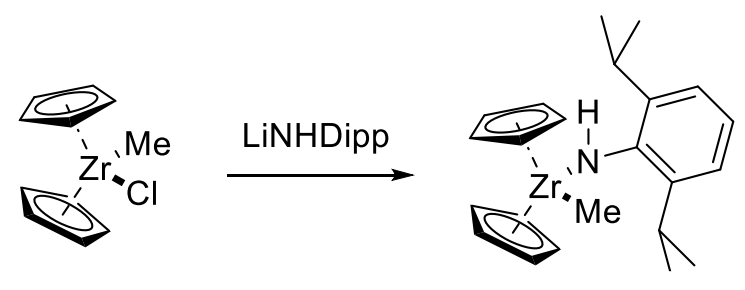

14

The ${ }^{1} \mathrm{H}$ NMR spectrum shows a chemical shift upfield for the methyl protons to $\delta 0.23 \mathrm{ppm}$ (from $\delta 0.45$ $\mathrm{ppm})$, likely due to reduced deshielding of the amide relative to the chloride. The isopropyl resonances of the amide are both shifted downfield from the free amine, to $\delta 3.41$ and $\delta 1.24 \mathrm{ppm}$ for the methine septet and methyl doublet respectively. The cyclopentadienyl resonance is upfield shifted to $\delta 5.63 \mathrm{ppm}$ and the amide proton is observable as a broad singlet at $\delta 6.21 \mathrm{ppm}$, significantly chemical shifted downfield from the free amine likely due to the increased deshielding though proximity to the zirconium atom. The meta substituted protons on the phenyl ring are observed as a doublet at $7.16 \mathrm{ppm}$ (the $\mathrm{J}_{H H}$ coupling constant could not be determined due to overlap with the solvent signal) and a triplet for the para substituted proton at $\delta 7.11 \mathrm{ppm}$. 
Crystals suitable for an X-ray diffraction study were obtained from a concentrated toluene solution of 13. As there are no suitable solid-state comparisons in the literature for 13 , analysis of the solid-state crystal structure data is carried out compared to $\mathbf{1 4}$ and $\mathbf{1 5}$ later in this chapter.

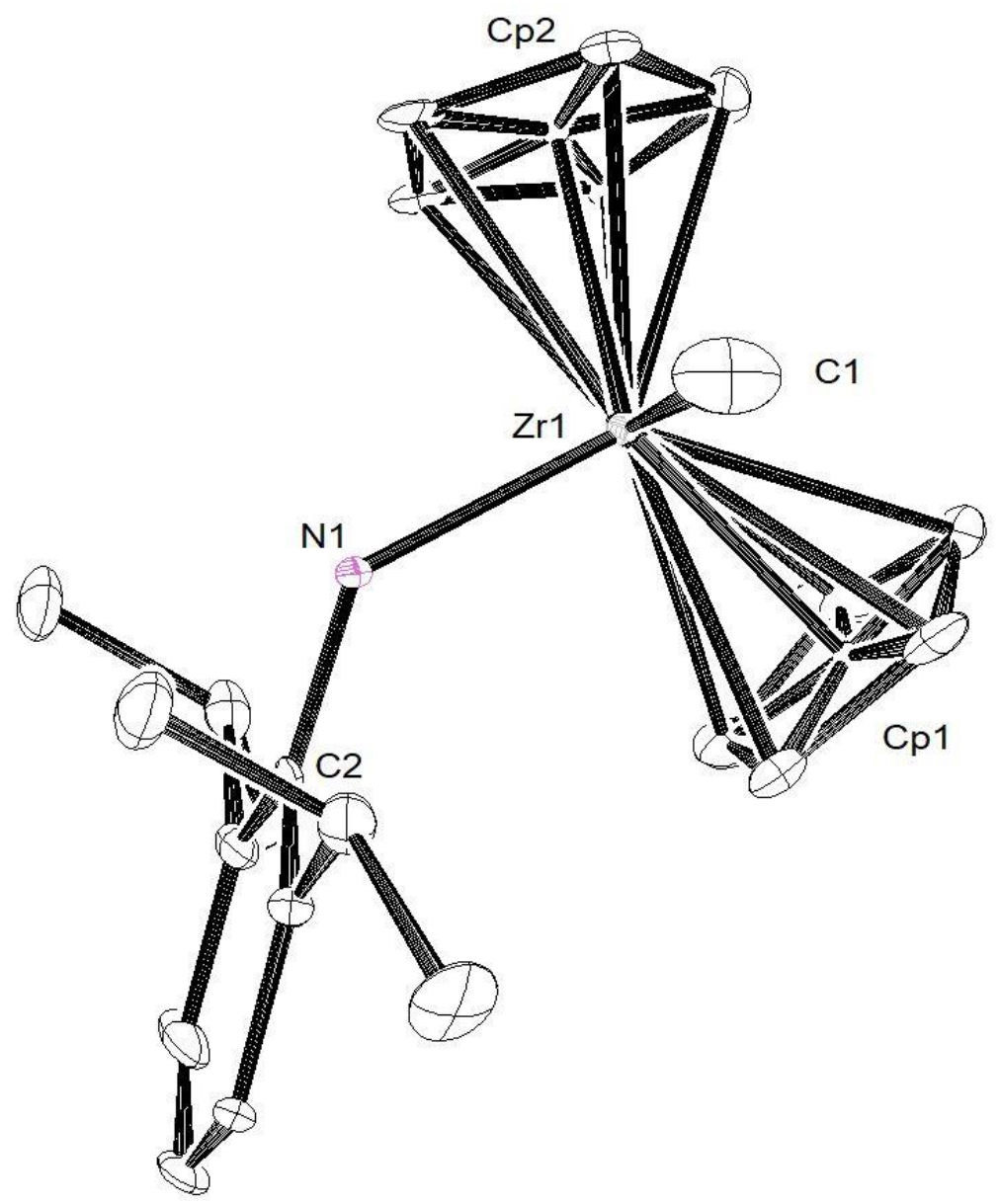

Figure 41: ORTEP representation of $14.30 \%$ Thermal ellipsoids. Hydrogens omitted for clarity.

Table 18: Selected bond lengths and angles of 14 .

\begin{tabular}{lccr}
\multicolumn{2}{c}{ Bond Lengths (Å) } & \multicolumn{2}{c}{ Bond Angles ( $\left.{ }^{\circ}\right)$} \\
$\operatorname{Zr}(1)-\mathrm{N}(1)$ & $2.080(9)$ & $\mathrm{Zr}(1)-\mathrm{N}(1)-\mathrm{C}(2)$ & $134.7(7)$ \\
$\operatorname{Zr}(1)-\mathrm{C}(1)$ & $2.345(18)$ & $\mathrm{N}(1)-\operatorname{Zr}(1)-\mathrm{C}(1)$ & $98.2(7)$ \\
$\operatorname{Zr}(1)-\mathrm{Cp}(2)$ & $2.228(8)$ & $\mathrm{Cp}(1)-\operatorname{Zr}(1)-\mathrm{Cp}(2)$ & $136.5(4)$ \\
$\operatorname{Zr}(1)-\mathrm{Cp}(1)$ & $2.220(6)$ & $\mathrm{Cp}(1)-\operatorname{Zr}(1)-\mathrm{C}(1)$ & $103.8(6)$ \\
& & $\mathrm{Cp}(2)-\operatorname{Zr}(1)-\mathrm{C}(1)$ & $96.5(8)$
\end{tabular}




\subsubsection{Synthesis of $\left[\mathrm{Cp}_{2} \mathrm{Zr}(\mathrm{Me})\left(\mathrm{NHAr}^{*}\right)\right] \quad 15$}

The amidozirconocene $\left[\mathrm{Cp}_{2} \mathrm{Zr}(\mathrm{Me})\left(\left(\mathrm{NHAr}^{*}\right)\right]\right.$ was synthesised from the addition of the lithiated amine [LiNHAr*] dropwise to $\left[\mathrm{Cp}_{2} \mathrm{Zr}(\mathrm{Cl})(\mathrm{Me})\right]$ in diethyl either at $-30{ }^{\circ} \mathrm{C}$ (equation 23). Following workup the compound was purified by crystalisation from toluene layered with hexane at $-30^{\circ} \mathrm{C}$. The crystalline solid was characterised by ${ }^{1} \mathrm{H} N M R,{ }^{13} \mathrm{C} N M R$ and single crystal X-ray spectroscopy.

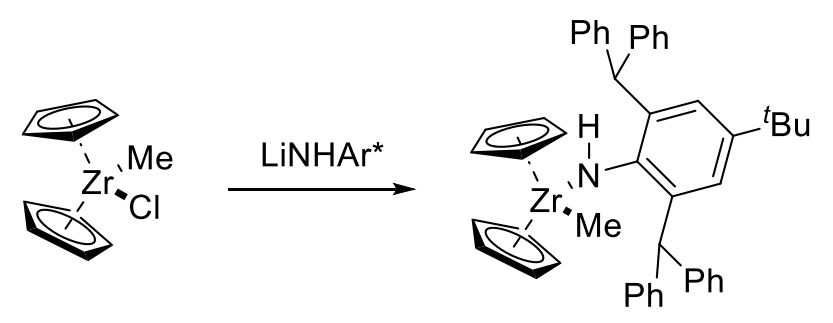

15

The ${ }^{1} \mathrm{H}$ NMR spectrum has a broad singlet at $\delta 7.24$ integrating to 8 protons as well as a broad singlet at $\delta$ $6.01\left(\mathrm{CHPh}_{2}\right)$ integrating for 2 protons, indicating a possible dynamic species in solution at room temperature. The other aromatic protons resonances at $\delta 7.19$ and a multiplet of overlapping signals at $\delta$ 7.06-6.99 ppm. The cyclopentadienyl resonance is upfield shifted to $\delta 5.58 \mathrm{ppm}$ from $\delta 5.71 \mathrm{ppm}$, indicating increased shielding of the cyclopentadienyl rings from the starting material. The amide proton peak is observable as a broad singlet at $\delta 2.79 \mathrm{ppm}$, while the singlet resonance attributed to the tertbutyl environment is shifted to $\delta 1.09 \mathrm{ppm}$, upfield from the free amine in deuterated benzene ( $\delta 1.11$ ppm). The broad singlet could be due to the inequivalence of some of the phenyl rings on the NHAr* amide ligand, due to the large steric bulk restricting free rotation. If this were the case, heating the compound up would expect to sharpen the peaks up (as the energy related to overcoming the energetic rotation barrier is overcome), while cooling the temperature of the NMR probe down might allow the two dynamic species to be isolated. 
Crystals suitable for an X-ray diffraction study were obtained from a concentrated toluene solution of 14. As discussed earlier analysis of the solid-state crystal structure is carried out later in this chapter.

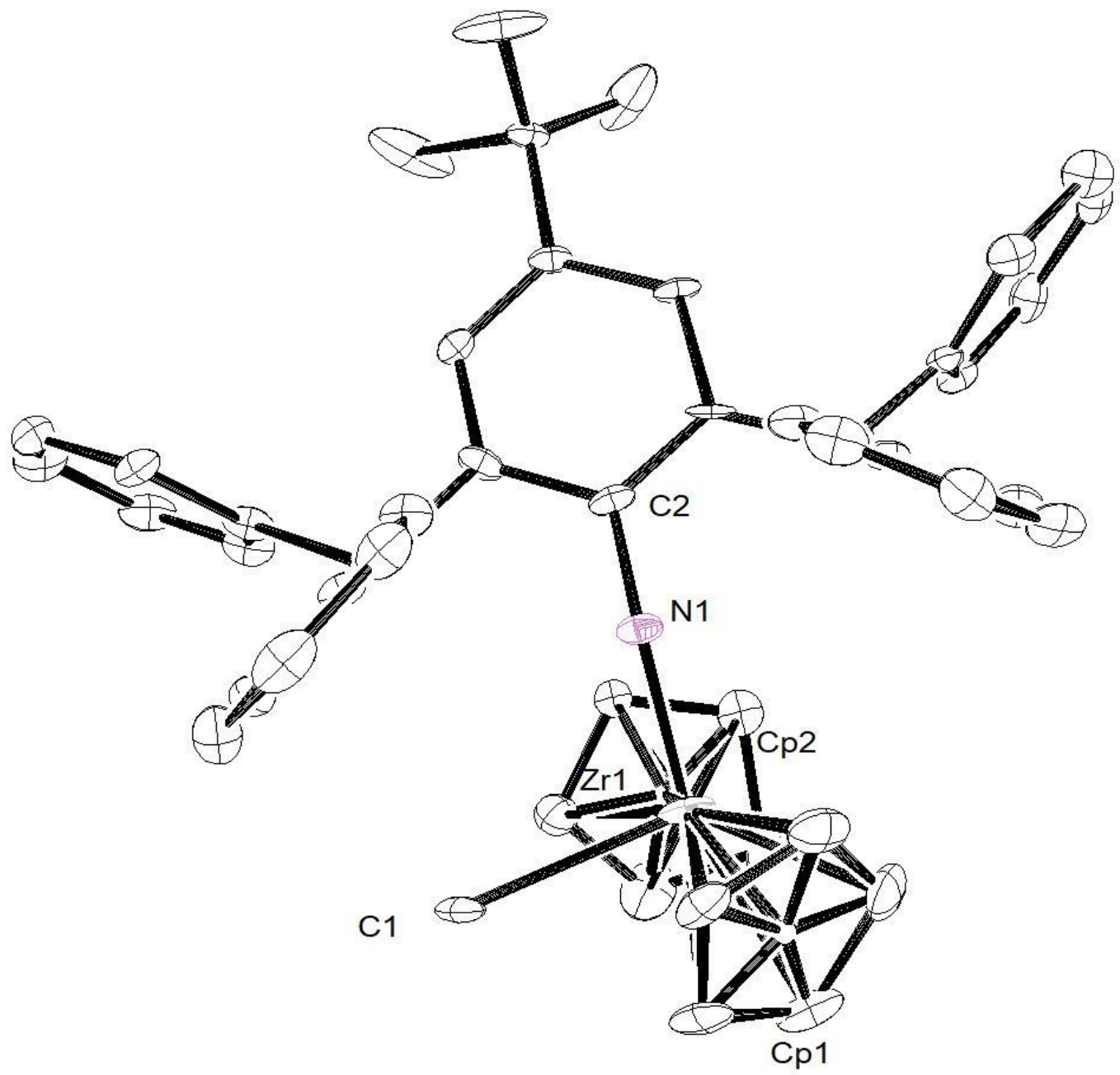

Figure 42: ORTEP representation of $15.30 \%$ Thermal ellipsoids. Hydrogens omitted for clarity.

Table 19: Selected bond lengths and angles of 15.

Bond Lengths ( $\AA$ )

$\begin{array}{cc}\operatorname{Zr}(1)-\mathrm{N}(1) & 2.080(3) \\ \mathrm{N}(1)-\mathrm{C}(2) & 1.412(5) \\ \mathrm{Cp}(1)-\operatorname{Zr}(1) & 2.2316(17) \\ \mathrm{Cp}(2)-\operatorname{Zr}(1) & 2.2251(17) \\ \operatorname{Zr}(1)-\mathrm{C}(1) & 2.303(4)\end{array}$

Bond Angles ( ${ }^{\circ}$ )

$\begin{array}{lc}\mathrm{Cp}(1)-\operatorname{Zr}(1)-\mathrm{N}(1) & 109.15(10) \\ \mathrm{Cp}(2)-\operatorname{Zr}(1)-\mathrm{N}(1) & 105.42(10) \\ \mathrm{Cp}(1)-\operatorname{Zr}(1)-\mathrm{C}(2) & 104.78(15) \\ \mathrm{Cp}(2)-\operatorname{Zr}(1)-\mathrm{C}(2) & 103.85(17) \\ \mathrm{Zr}(1)-\mathrm{N}(1)-\mathrm{C}(2) & 134.9(2) \\ \mathrm{C}(1)-\operatorname{Zr}(1)-\mathrm{N}(1) & 99.94(15) \\ \mathrm{Cp}(1)-\operatorname{Zr}(1)-\mathrm{Cp}(2) & 130.51(8)\end{array}$




\subsubsection{Synthesis of $\left[\mathrm{Cp}_{2} \mathrm{Zr}(\mathrm{Me})(\mathrm{NH}(\mathrm{DMP}))\right] \quad 16$}

$\left[\mathrm{Cp}_{2} \mathrm{Zr}(\mathrm{Me}) \mathrm{NH}(\mathrm{DMP})\right]$ (DMP = 2,6 dimethylphenyl) was synthesised using two different synthetic routes. The first was the through salt metathesis of the lithiated amine with $\left[\mathrm{Cp}_{2} \mathrm{Zr}(\mathrm{Cl})(\mathrm{Me})\right]$, while the second was the treatment of $\left[\mathrm{Cp}_{2} \mathrm{ZrMe}_{2}\right]$ with the free amine at $110{ }^{\circ} \mathrm{C}$ (Scheme 26). For both reactions, the product was purified after workup by crystallisation. Compound 16 was characterised by ${ }^{1} \mathrm{H}$ NMR spectroscopy, ${ }^{13} \mathrm{C}$ NMR spectroscopy, single crystal X-ray diffraction. Unlike many earlier compounds elemental analysis was able to be obtained accurately for $\mathbf{1 6 .}$

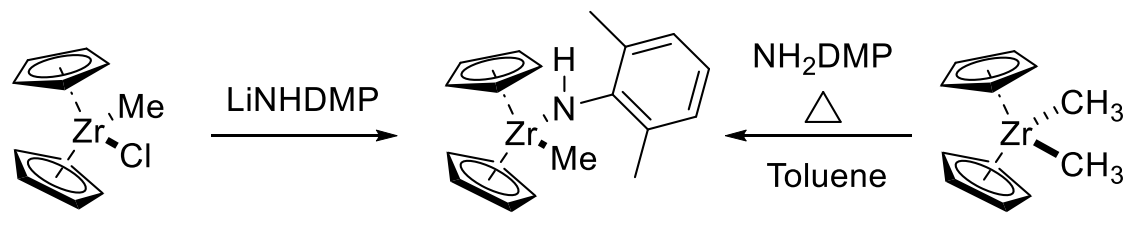

16

Scheme 26: Synthesis of 16 from transamination and salt metathesis reactions.

The ${ }^{1} \mathrm{H}$ NMR spectrum of 16 demonstrated one aromatic multiplet resonances for the meta and para protons on the DMP ring at $\delta$ 7.11-7.07 ppm (3H). The cyclopentadienyl resonance at $\delta 5.34 \mathrm{ppm}(10 \mathrm{H})$ has a chemical shift upfield from either starting material, and is also upfield of both $\mathbf{1 4}$ and $\mathbf{1 5}$. This could be due to a steric or electronic resulting from the different substitution of the meta positions of the phenylamide ring. The 2,6 methyl environments resonate at $\delta 2.04 \mathrm{ppm}(6 \mathrm{H})$ and have a chemical shift downfield compared to the free amine resonance of $\delta 1.88 \mathrm{ppm}$. The zirconium-methyl resonance at $\delta$

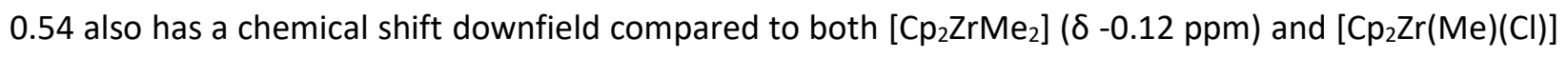
( $\delta 0.45 \mathrm{ppm}$ ), indicating a change to a more deshielded chemical environment.

Crystals suitable for an X-ray diffraction study were obtained from a concentrated toluene solution of 16. Analysis of the solid-state crystal structure is carried out later in this chapter (Section 3.1.5). 


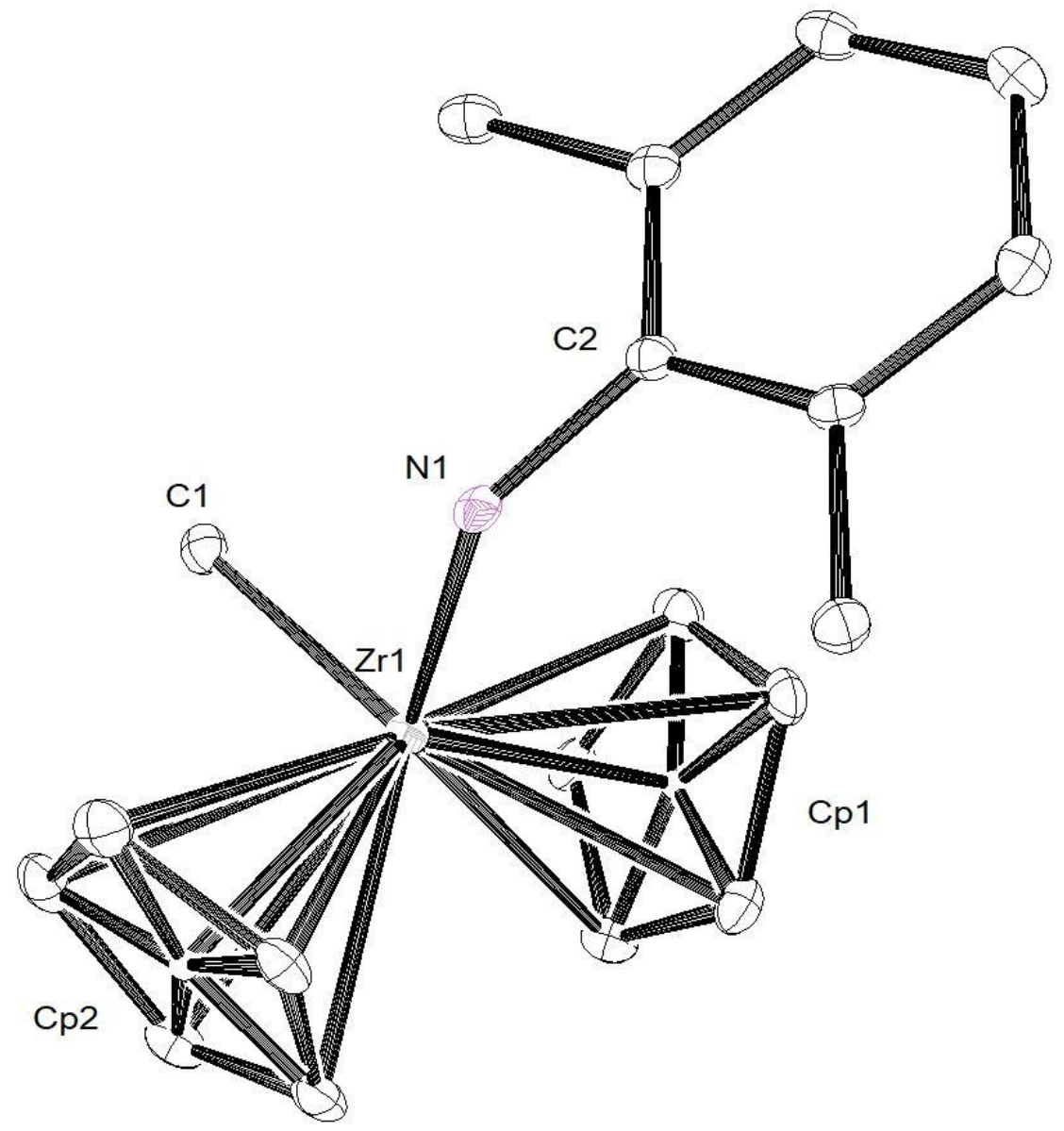

Figure 43: ORTEP representation of $16.30 \%$ Thermal ellipsoids. Hydrogens omitted for clarity.

Table 20: Selected bond lengths and angles of 16.

\begin{tabular}{|c|c|c|c|}
\hline \multicolumn{2}{|c|}{ Bond Lengths (Å) } & \multicolumn{2}{|c|}{ Bond Angles $\left({ }^{\circ}\right)$} \\
\hline $\mathrm{Zr}(1)-\mathrm{N}(1)$ & $2.078(3)$ & $\mathrm{Zr}(1)-\mathrm{N}(1)-\mathrm{C}(2)$ & $139.6(2)$ \\
\hline $\operatorname{Zr}(1)-C(1)$ & $2.526(5)$ & $N(1)-\operatorname{Zr}(1)-C(1)$ & $98.8(2)$ \\
\hline $\operatorname{Zr}(1)-\operatorname{Cp}(2)$ & $2.156(2)$ & $C p(1)-Z r(1)-C p(2)$ & $141.88(11)$ \\
\hline $\operatorname{Zr}(1)-C p(1)$ & $2.205(2)$ & $C p(1)-\operatorname{Zr}(1)-C(1)$ & $88.23(15)$ \\
\hline & & $C p(2)-Z r(1)-C(1)$ & $99.08(14)$ \\
\hline
\end{tabular}

\subsubsection{Comparison of solid-state crystal structures 14, 15 and 16}

The solid-state crystal structures of the amides 13,14 and 15 all have a distorted tetrahedral geometry around the $\mathrm{Zr}(1)$ atom. The $\mathrm{Zr}-\mathrm{N}$ bond lengths are extremely similar for all three compounds (2.080(9) $\AA$, 
2.078(3) Å and 2.080(3) $\AA$ for 14, 15 and 16 respectively), which would indicate that the steric bulk at the ortho positions of the aryl amides has little effect on the length of the $\mathrm{Zr}-\mathrm{N}$ bond. The data indicates that the $\operatorname{Zr}(1)-C(1)$ zirconium-methyl bond distance increases as the steric bulk of the aryl amide is increased. This effect is quite pronounced, with 15 having a $\operatorname{Zr}(1)-C(1)$ bond distance over $0.2 \AA$ longer than complex 16. This effect could be due to a number of different reasons, including solid state crystal packing effects, lengthening due to the increased steric bulk of the amide or a complicated electronic effect due the differing inductive effects of the substituents on the ortho positions (and possibly para position) of the phenyl ring of the amide. The $\operatorname{Zr}(1)-\mathrm{N}(1)-\mathrm{C}(2)$ bond angle of $15\left(139.6(2)^{\circ}\right)$ is significantly more distorted than that in 14 and $16\left(134.9(2)^{\circ}\right.$ and $134(7)^{\circ}$ respectively), indicating that as the steric bulk is increased the angle between these atoms increases, which could be expected to minimise steric interaction between the ligands.

\subsubsection{Synthesis of $\left[\mathrm{Cp}_{2} \mathrm{Zr}(\mathrm{NHPh})(\mathrm{Bn})\right] \quad 17$}

The amide $\left[\mathrm{Cp}_{2} \mathrm{Zr}(\mathrm{NHPh})(\mathrm{Bn})\right]$ was synthesised through treatment of bisbenzylzirconocene with aniline at elevated temperatures (eq. 24). This reaction gave a mixture of the products 17 and $\mathbf{1 8}$, although as 18 is an insoluble green solid the ${ }^{1} \mathrm{H}$ NMR spectrum of the unpurified reaction solution indicated $\mathbf{1 7}$ was the only product in solution.

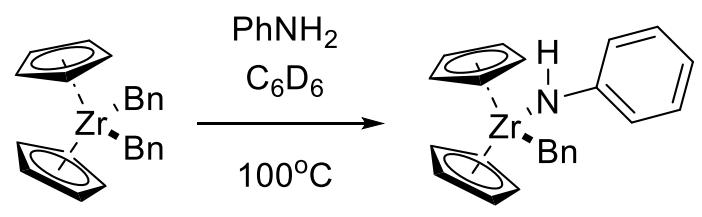

17

The ${ }^{1} \mathrm{H}$ NMR spectrum of $\mathbf{1 7}$ shows a number of overlapping signals in the aromatic region of the spectrum that are assigned as multiplets $\delta 7.26-7.19$ and $6.90-6.86 \mathrm{ppm}$. The doublets at $\delta 7.06(2 \mathrm{H})$ and $6.76 \mathrm{ppm}$ $(2 \mathrm{H})$ are likely the ortho substituted protons for each of the phenyl rings. Assignment for each ring resonance was not determined, however, one could speculate that the more downfield shifted resonance would be expected to be from the amide phenyl ring due the expected increased deshielding effect of the nitrogen due to increased electronegativity of carbon over the benzyl. The cyclopentadienyl resonance at $\delta 5.51 \mathrm{ppm}$ is not shifted from the bis-benzylzirconocene, indicating similar shielding on the cyclopentadienyl ligands. However, the resonance at $\delta 2.24 \mathrm{ppm}(2 \mathrm{H})$ indicates that there has been a 
change on the methylene environment on the benzyl fragment, which provides evidence that the amide has been synthesised.

Crystals suitable for an X-ray diffraction study were obtained from a concentrated hexane solution of 17. The crystal structure reveals a single molecule of 17 in the asymmetric unit. $\operatorname{The} \operatorname{Zr}(1)-\mathrm{N}(1)$ bond distance of 2.112(5) $\AA$ is longer than that of the 14 (2.080(9) $\AA$ ), 15 (2.078(3) $\AA$ ) and 16 (2.080(3) $\AA$ ), exhibiting the different electronic and steric profile of the benzyl ligand compared to the methyl ligand. Comparison of the solid-state structure of $\mathbf{1 7}$ with $\left[\mathrm{Cp}_{2} \mathrm{Zr}(\mathrm{Me})(\mathrm{NHPh})\right]$ would have given more insight into the effect of the benzyl ligand, however, despite several attempts the synthesis and isolation of [ $\left.\mathrm{Cp}_{2} \mathrm{Zr}(\mathrm{Me})(\mathrm{NHPh})\right]$ was not successful. The $\operatorname{Zr}(1)-C(1)$ bond length of $2.324(7) \AA$ falls within the range of $\operatorname{Zr}(1)-C(1)$ bond distances of 14, 15 and 16 (2.303(4)-2.526(5) $\AA$ ), indicating that the $\mathrm{Zr}-\mathrm{C}$ bond length of arylamidozirconocenes with a benzyl ligand is longer than the equivalent complex with a methyl ligand. The angle between the centroids of the cyclopentadienyl ligands is smaller in $\mathbf{1 7}\left(128.82(7)^{\circ}\right)$ than in the methyl complexes 14,15 and $16\left(136.5(4)^{\circ}, 141.88(11)^{\circ}\right.$ and $134.9(2)^{\circ}$ respectively). This is likely due to the increased steric profile of the benzyl ligand compared to the methyl ligand.

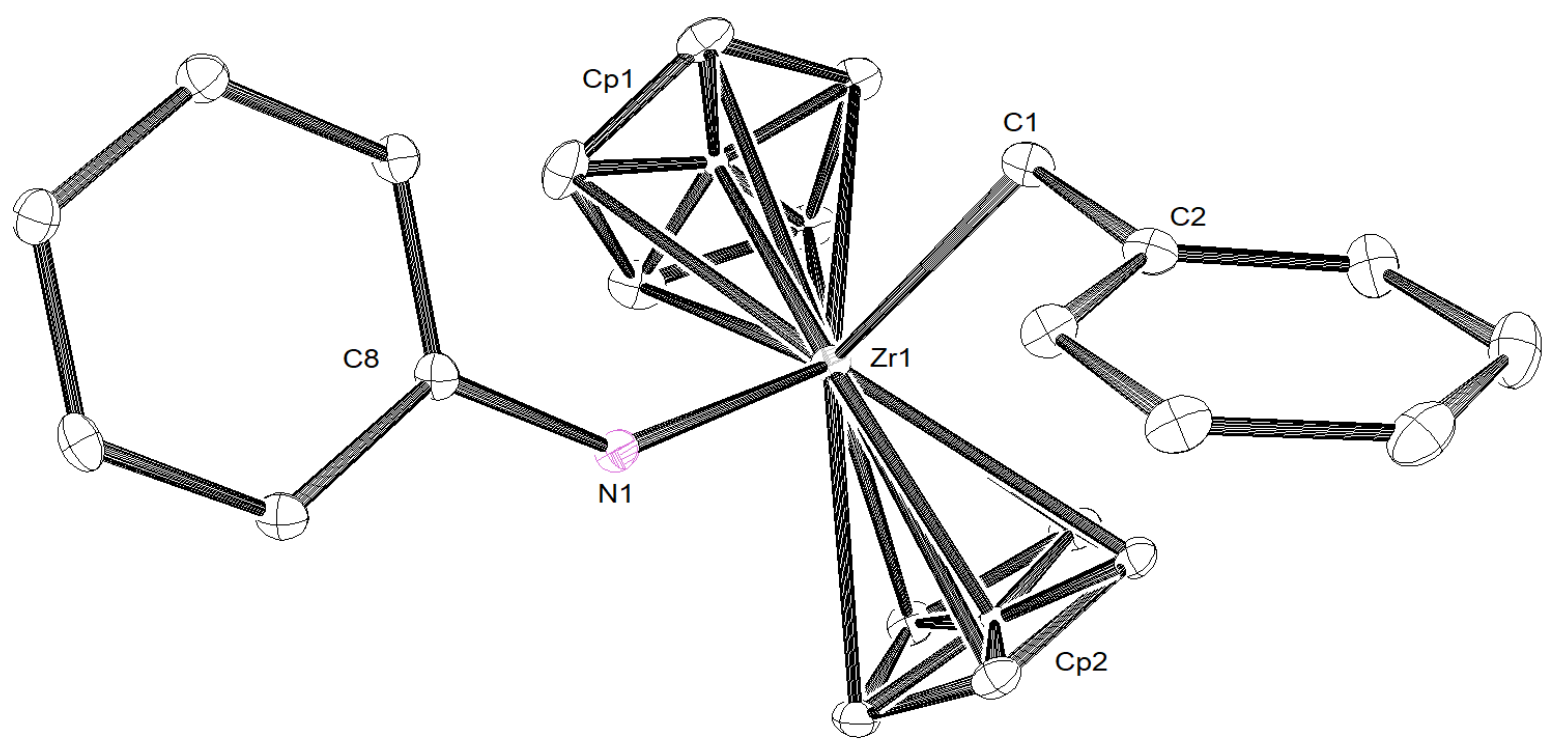

Figure 44: ORTEP representation of $17.30 \%$ Thermal ellipsoids. Hydrogens omitted for clarity. 
Table 21: Selected bond lengths and angles of 17.

\section{Bond Lengths ( $\AA$ )}

$\begin{array}{ll}\operatorname{Zr}(1)-\mathrm{N}(1) & 2.112(5) \\ \operatorname{Zr}(1)-\mathrm{C}(1) & 2.324(7) \\ \operatorname{Zr}(1)-\mathrm{Cp}(1) & 2.229(3) \\ \operatorname{Zr}(1)-\mathrm{Cp}(2) & 2.207(3) \\ \mathrm{N}(1)-\mathrm{C}(8) & 1.394(8) \\ \mathrm{C}(1)-\mathrm{C}(2) & 1.498(9)\end{array}$

Bond Angles ( $\left.{ }^{\circ}\right)$

$\begin{array}{cc}\operatorname{Zr}(1)-\mathrm{N}(1)-\mathrm{C}(8) & 140.8(4) \\ \operatorname{Zr}(1)-\mathrm{C}(1)-\mathrm{C}(2) & 120.6(4) \\ \mathrm{Cp}(1)-\operatorname{Zr}(1)-\mathrm{Cp}(2) & 128.82(12) \\ \mathrm{N}(1)-\operatorname{Zr}(1)-\mathrm{C}(1) & 96.2(2)\end{array}$

\subsubsection{Synthesis of $\left[\left(\mathrm{Cp}_{2} \mathrm{Zr}(\mu-\mathrm{NPh})_{2}\right)\right] \quad 18$}

The amide bridged zirconium dimer 18 was synthesised from the addition of free aniline to bisbenzylzirconocene in deuterated benzene at $100{ }^{\circ} \mathrm{C}$. After heating at for 7 days insoluble green crystals were obtained as the major product. Analysis by ${ }^{1} \mathrm{H}$ NMR spectroscopy and ${ }^{13} \mathrm{C}$ NMR spectroscopy was not possible due to the insolubility of the crystals in all available solvents.

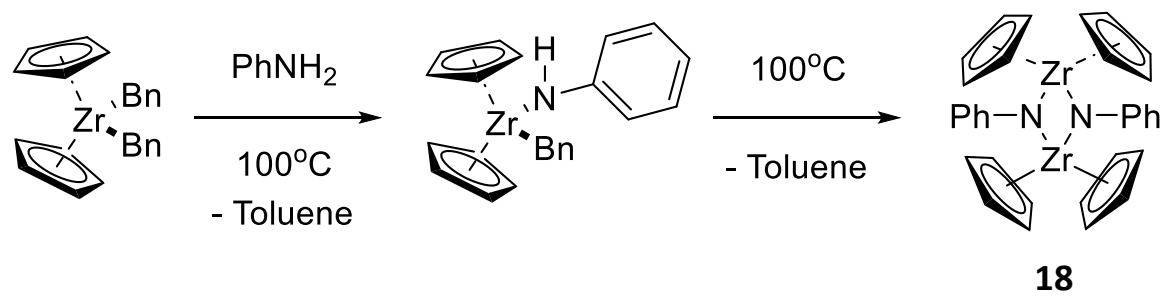

Scheme 27: Synthesis of 16 from transamination and salt metathesis reactions.

The green crystals were submitted for an X-ray diffraction study. However due to inadequate refinement and modelling the crystal structure data is not deemed to be of acceptable quality to be discussed in this thesis (the refinement data and ORTEP diagram are reported in the appendices). The crystal structure data indicates the synthesis of the amido-bridged dimer $\left[\left(\mathrm{Cp}_{2} \mathrm{Zr}(\mu-\mathrm{NPh})_{2}\right)\right]$. The crystal structure data for 18 shows the zirconium atoms and bridging phenylamide ligand is one plane, with two cyclopentadienyl ligands bonded to each zirconium atom, one located above and one below this plane. The structure of 18 is extremely similar to the literature compound XXIII (including significant disorder of the crystal structure) and is also likely formed due to the lack of steric bulk at the meta positions of the phenylamide. 


\subsubsection{Synthesis of $\left[C p_{2} Z r(N H D M P)(B n)\right] 19$}

The amide $\left[\mathrm{Cp}_{2} \mathrm{Zr}(\mathrm{NHDMP})(\mathrm{Bn})\right], 19$ was synthesised through treatment of bisbenzylzirconocene with dimethylphenylaniline at elevated temperatures (eq. 25). Compound 19 was characterised by ${ }^{1} \mathrm{H}$ NMR spectroscopy, ${ }^{13} \mathrm{C}$ NMR spectroscopy and single crystal X-ray diffraction.

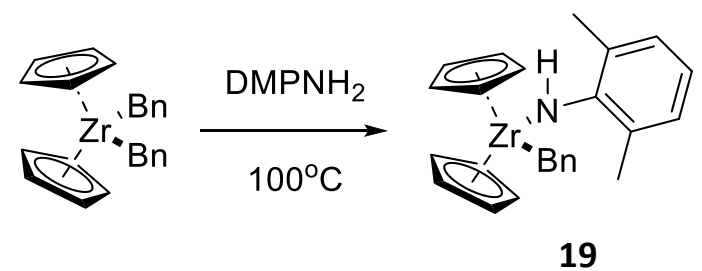

The ${ }^{1} \mathrm{H}$ NMR spectrum of 19 revealed 5 aromatic signals, a triplet at $\delta 7.34 \mathrm{ppm}(2 \mathrm{H})$, a doublet at $\delta 7.22$ ppm (2H), a multiplet at $\delta 7.11 \mathrm{ppm}(2 \mathrm{H})$, a triplet at $\delta 7.05 \mathrm{ppm}(1 \mathrm{H})$ and a triplet at $\delta 6.95 \mathrm{ppm}(1 \mathrm{H})$. The aromatic resonances were not assigned to specific hydrogen environments but one would assume the two triplets at $\delta 7.05$ and $6.95 \mathrm{ppm}$ and from the para substituted protons of the NHDMP and Bn ligands respectively. The cyclopentadienyl environment is attributed to the resonance at $\delta 5.44 \mathrm{ppm}(10 \mathrm{H})$, chemical shifted upfield from bisbenzylzirconocene $(\delta 5.51 \mathrm{ppm})$. The methylene resonance of the benzyl ligand is chemical shifted downfield from $\delta 1.83 \mathrm{ppm}(4 \mathrm{H})$ to $\delta 2.25 \mathrm{ppm}(2 \mathrm{H})$. The ortho substituted methyl environments of the NHDMP ligand are observed as a resonance at $\delta 2.14 \mathrm{ppm}$, chemical shifted downfield from the free amine ( $\delta 1.88 \mathrm{ppm})$.

Crystals suitable for an X-ray diffraction study were obtained from a concentrated hexane solution of 19. The crystal structure reveals a single molecule of 19 in the asymmetric unit as well as a molecule of toluene at half occupancy that was determined not to be interacting with the molecule of 19 . The $\operatorname{Zr}(1)-\mathrm{N}(1)$ bond distance of 2.087(2) $\AA$ is longer than that of the methyl complexes 14 (2.080(9) $\AA$ ), 15 (2.078(3) $\AA$ ) and 16 $(2.080(3) \AA ̊)$, indicating that the different electronic and steric profile of the benzyl ligand compared to the methyl ligand, however the small difference between the complexes makes doesn't make the differences significant. $\operatorname{The} \operatorname{Zr}(1)-C(9)$ bond length of 2.355(3) A falls within the range of $\operatorname{Zr}(1)-C(1)$ bond distances of 14,15 and 16 (2.303(4)-2.526(5) $\AA$ ), indicating that the Zr-C bond length of arylamidozirconocenes with a benzyl ligand is longer than the equivalent complex with a methyl ligand. The angle between the centroids of the cyclopentadienyl ligands is much smaller in $\mathbf{1 7}\left(130.88(7)^{\circ}\right)$ than in the methyl complexes 14, 15 and $16\left(136.5(4)^{\circ}, 141.88(11)^{\circ}\right.$ and $134.9(2)^{\circ}$ respectively), but larger than the benzyl complex 17 $\left(128.82(12)^{\circ}\right)$. The $\mathrm{Zr}-\mathrm{N}(1)-\mathrm{C}(1)$ angle of $137.13^{\circ}$ is smaller than that of the $\operatorname{Zr}(1)-\mathrm{N}(1)-\mathrm{C}(8)$ angle of 
$140.8(4)^{\circ}$ in 17 , indicating that as the steric bulk at the ortho positions of the phenylamide rings is increased this angle decreases. This is in contrast to the effect observed in the methyl complexes 14, 15 and 16.

Table 22: Selected bond lengths and angles of 19.

\begin{tabular}{|c|c|c|c|}
\hline \multicolumn{2}{|c|}{ Bond Lengths ( $(\AA)$} & \multicolumn{2}{|c|}{ Bond Angles $\left({ }^{\circ}\right)$} \\
\hline $\operatorname{Zr}(1)-N(1)$ & $2.087(2)$ & $\operatorname{Zr}(1)-N(1)-C(1)$ & $137.13(16)$ \\
\hline $\operatorname{Zr}(1)-C(9)$ & $2.355(3)$ & $\operatorname{Zr}(1)-C(9)-C(10)$ & $113.21(17)$ \\
\hline $\operatorname{Zr}(1)-C p(1)$ & $2.212(3)$ & $N(1)-\operatorname{Zr}(1)-C p(1)$ & $106.91(11)$ \\
\hline \multirow[t]{3}{*}{$\operatorname{Zr}(1)-C p(2)$} & $2.284(13)$ & $N(1)-\operatorname{Zr}(1)-C p(2)$ & $108.31(7)$ \\
\hline & & $C(9)-\operatorname{Zr}(1)-C p(1)$ & $104.61(11)$ \\
\hline & & $C(9)-\operatorname{Zr}(1)-C p(2)$ & $130.88(7)$ \\
\hline
\end{tabular}

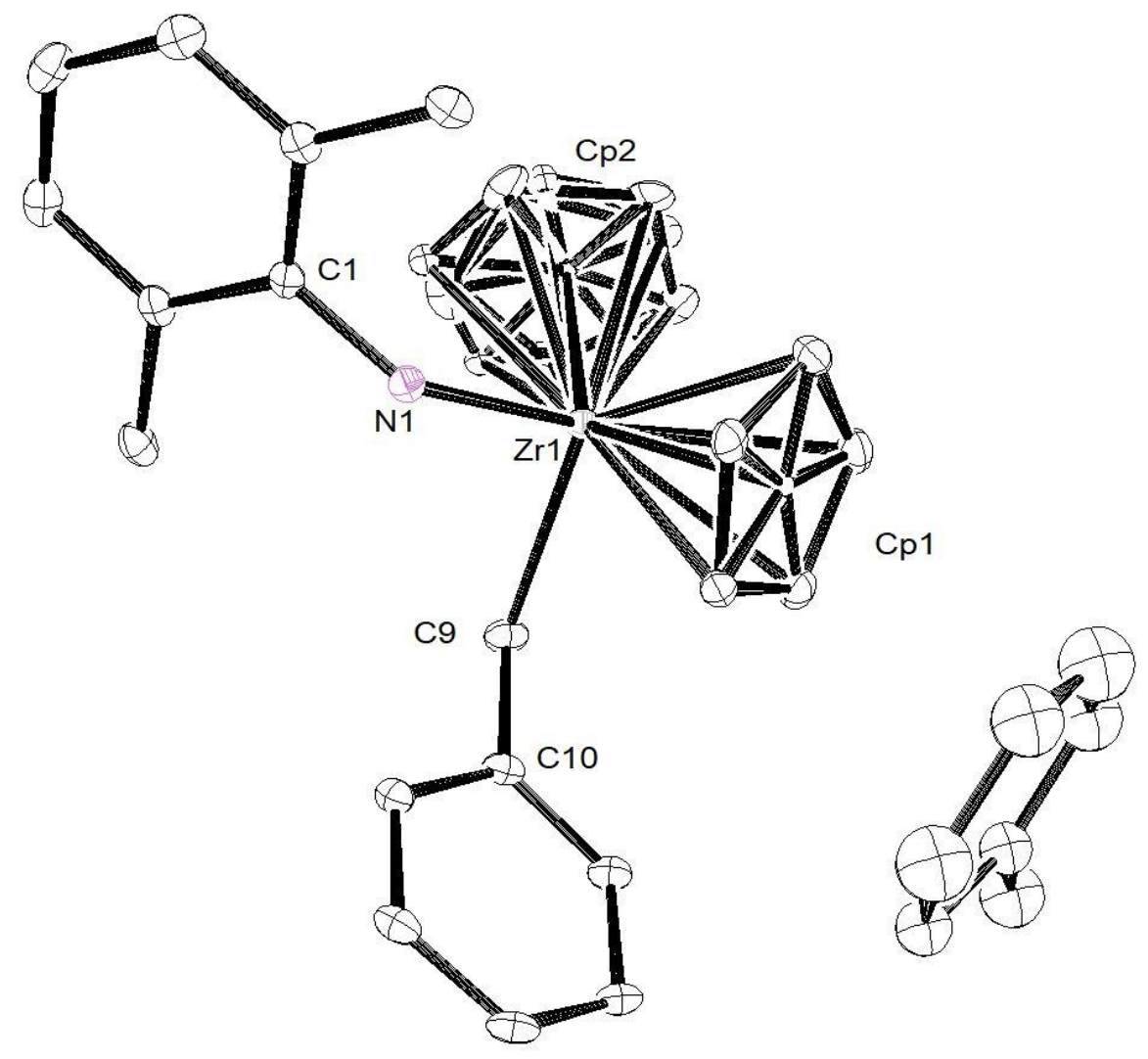

Figure 45: ORTEP representation of $19.30 \%$ Thermal ellipsoids. Hydrogens omitted for clarity. Disorder in the $\mathrm{Cp} 2$ ring modelled over two positions. 


\subsubsection{Synthesis of $\left[\mathrm{Cp}_{2} \mathrm{Zr}(\mathrm{NAr} *)(\mathrm{THF})\right] \quad 20$}

$\left[\mathrm{Cp}_{2} \mathrm{Zr}\left(\mathrm{NAr}{ }^{*}\right)(\mathrm{THF})\right](\mathbf{2 0})$ was synthesised from the thermolysis of 15 at $100{ }^{\circ} \mathrm{C}$ in the presence of THF. The orange crystals were characterised by ${ }^{1} \mathrm{H}$ NMR spectroscopy, ${ }^{13} \mathrm{C}$ NMR spectroscopy and single crystal Xray diffraction.
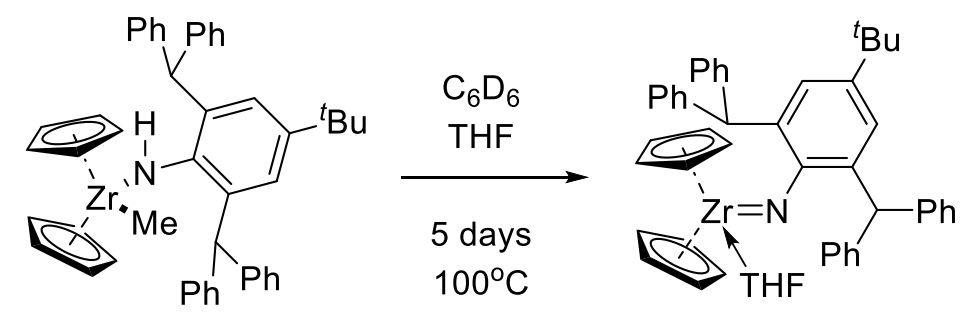

20

The ${ }^{1} \mathrm{H}$ NMR spectrum of $\mathbf{2 0}$ indicated all four of the flanking phenyl rings were equivalent, hence were observed as three resonances; a doublet $\delta 7.39 \mathrm{ppm}(8 \mathrm{H})$, a triplet at $\delta 7.20 \mathrm{ppm}(8 \mathrm{H})$ and a triplet of at $\delta 7.08 \mathrm{ppm}(4 \mathrm{H})$ correspond to the ortho, meta and para substituted protons respectively. The singlet at $\delta 7.05 \mathrm{ppm}(2 \mathrm{H})$ was attributed to the meta protons on the tetrasubstituted phenyl ring, while the singlet at $\delta 6.43 \mathrm{ppm}(2 \mathrm{H})$ was determined to be the resonance of the equivalent methine $(\mathrm{CH})$ protons. The singlet at $\delta 5.74 \mathrm{ppm}(10 \mathrm{H})$ was attributed to the cyclopentadienyl environment, a chemical shift downfield from the resonance in $15(\delta 5.58 \mathrm{ppm}(10 \mathrm{H}))$. The two THF multiplets integrate to 4 protons each, and are shifted from free THF to $\delta 3.44 \mathrm{ppm}$ and $\delta 1.08 \mathrm{ppm}$ respectively, indicating increased shielding likely due to coordination to the zirconium atom. ${ }^{71}$ There is no observable high field alkyl resonance or broad amide proton in the ${ }^{1} \mathrm{H}$ NMR spectrum, indicating the loss of the methyl as methane from 15.

Crystals suitable for an X-ray diffraction study were obtained from a concentrated toluene solution of 20. The X-ray crystal structure shows one molecule of $\mathbf{2 0}$ in the asymmetric unit along with one molecule of toluene and one molecule of THF acting as an electron donor to the zirconium atom. The toluene atom was determined not to be interacting with the solid-state structure $\mathbf{2 0}$. The crystal structure data shows a distorted tetrahedral geometry at the zirconium centre and has the unique almost linear $\mathrm{Zr}(1)-\mathrm{N}(1)-\mathrm{C}(1)$ bond angle of $167.60^{\circ}$, characteristic of the known complexes of this type. ${ }^{49,54-55,114}$ This angle is larger than compound $(\mathbf{X X X I}(\mathbf{a}))\left(157.17^{\circ}\right)$, but smaller than the known tert-butyl imidozirconocene $\mathbf{X X X}$ $\left(172.55^{\circ}, 174.32^{\circ}\right)$. The almost linear bond angle is attributed to the nitrogen acting as a four electron donor, lengthening the $\mathrm{Zr}-\mathrm{N}$ bond distance and formally making the species a 20 electron complex. ${ }^{49}$ The smaller angle in $\mathbf{2 0}$ relative to the literature indicates that the nitrogen may be acting less as a four 
electron donor than in XXXX. The Zr(1)-N(1) bond length of $1.891 \AA$ is longer than that of $\mathbf{X X X I ( a )}$ and $\mathbf{X X X}$ (1.889 and $1.826 \AA$ respectively), likely due to the increased steric profile of the NAr* ligand over the NDipp and $N^{t} B u$ ligands. ${ }^{115} \operatorname{The} \operatorname{Zr}(1)-O(1)$ bond distance of $2.300 \AA$ is longer than in the complexes $\mathbf{X X X}$ $(2.260 \AA)$ and $\mathbf{X X X ( a )}(2.240 \AA)$, likely also a reflection of the increased bulkiness of the NAr* ligand, though possibly a function of the ligands increased $\sigma$-donor ability relative to other bulky amines due to the increased carbon substitution at the 2,4 and 6 positions of the ligands central phenyl ring. The cyclopentadienyl-zirconium distances $(\mathrm{Cp}-\operatorname{Zr}(1))$ are $2.272 \AA$ and $2.289 \AA$ for the two cyclopentadenyl ligands, measured from the centre of the 5 carbon atoms to the zirconium atom. These distances are slightly longer than in that repeated in the literature for the compounds $\mathbf{X X X}$ and $\mathbf{X X X I ( a ) . ~}$ 


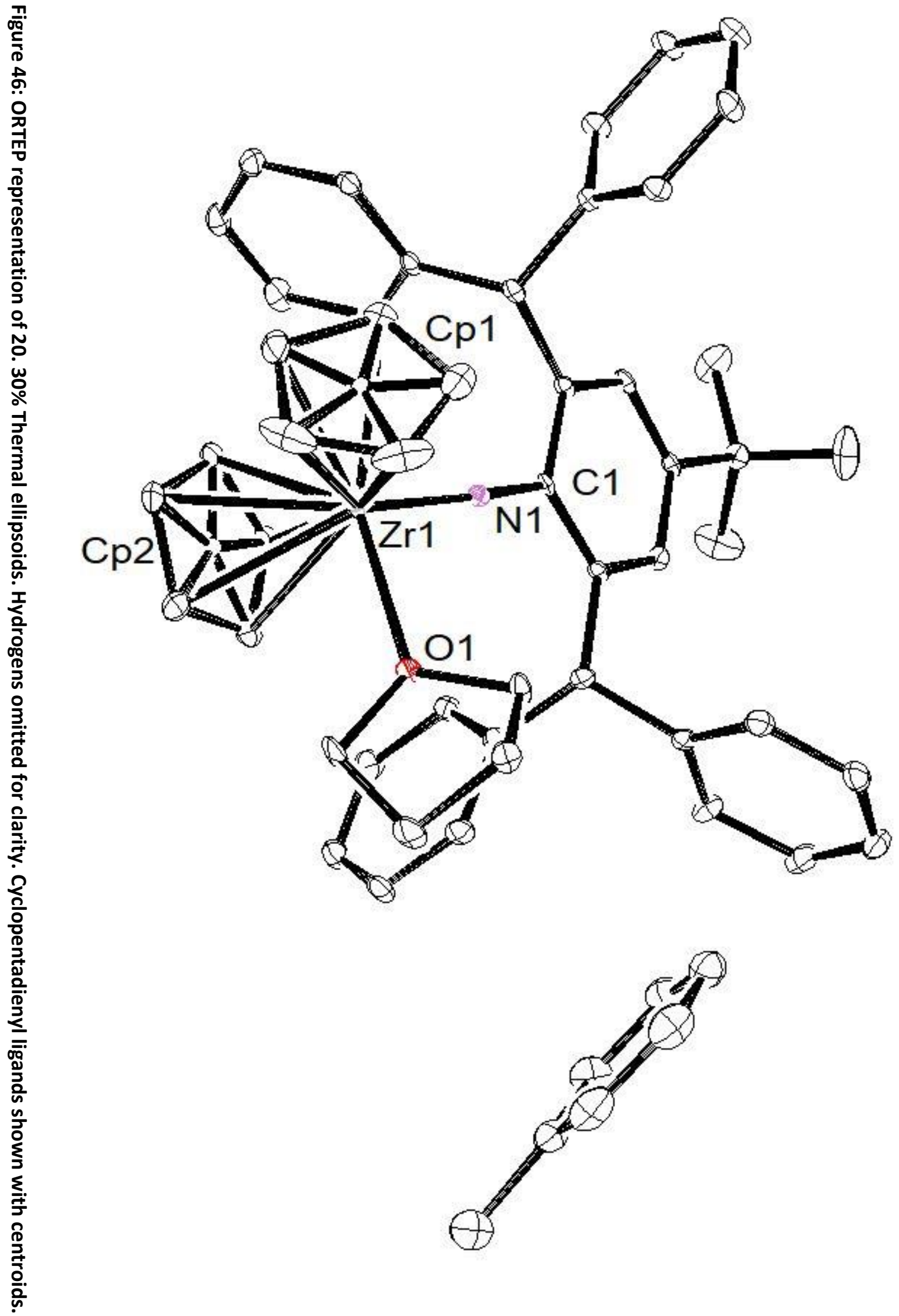


Table 23: Selected bond lengths and angles of 20.

Bond Lengths $(\AA)$

$\begin{array}{cc}\operatorname{Zr}(1)-\mathrm{N}(1) & 1.891 \\ \mathrm{~N}(1)-\mathrm{C}(1) & 1.365 \\ \mathrm{Zr}(1)-\mathrm{Cp}(1) & 2.289 \\ \mathrm{Zr}(1)-\mathrm{Cp}(2) & 2.272 \\ \operatorname{Zr}(1)-\mathrm{O}(1) & 2.300\end{array}$

Bond Angles ( $\left.{ }^{\circ}\right)$

$\begin{array}{cc}\operatorname{Zr}(1)-N(1)-C(1) & 167.60 \\ C p(1)-\operatorname{Zr}(1)-C p(2) & 124.89 \\ N(1)-\operatorname{Zr}(1)-O(1) & 95.91 \\ N(1)-\operatorname{Zr}(1)-C p(1) & 113.21 \\ N(1)-\operatorname{Zr}(1)-C p(2) & 110.30\end{array}$

\subsection{Insertion Reactions of Azides with alkylzirconocenes}

\subsubsection{Synthesis of $\left[\mathrm{Cp}_{2} \mathrm{Zr}(\mathrm{Bu})\left(\mathrm{N}_{3}(\mathrm{Bu})(\mathrm{Mes})\right)\right] \quad 21$}

The first route toward a zirconium nitrogen double bond was the synthesis of [ $\mathrm{Cp}_{2} \mathrm{Zr}=\mathrm{NMes}$ ]. The rationale behind the reaction was that upon generation of the Negishi reagent; ' $C p_{2} Z r$ ' a $\mathrm{Zr}(\mathrm{II})$ species, oxidative addition with an azide would result in formation of the nitrogen double bond with the elimination of nitrogen gas being a driving force behind bond formation. This synthetic strategy has been used for a wide range of other metals for the formation of nitrogen double bonds including $\mathrm{Fe}, \mathrm{Co}, \mathrm{Ge}, \mathrm{Sn}$ and Nb. ${ }^{41,116-118}$ 119

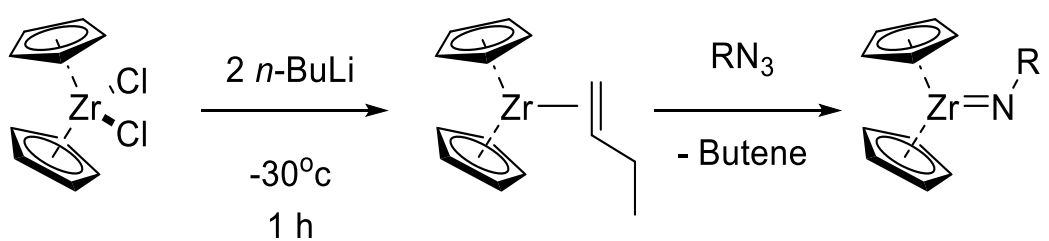

Scheme 28: Proposed synthetic route to imidozirconocenes from oxidative addition to the Negishi reagent.

However, when the reaction was carried out via the assumed generation of the Negishi reagent at $-30{ }^{\circ} \mathrm{C}$, upon addition of the azide the reaction gave the insertion product $\mathbf{2 1}$. The formation of the triazenido ligand from azides is known in the literature for zirconium, however, the majority of triazenido complexes with zirconium are synthesised via deprotonation of the corresponding triazene followed by coordination opposed to insertion into the zirconium-alkyl bond. ${ }^{55,120-121}$ 


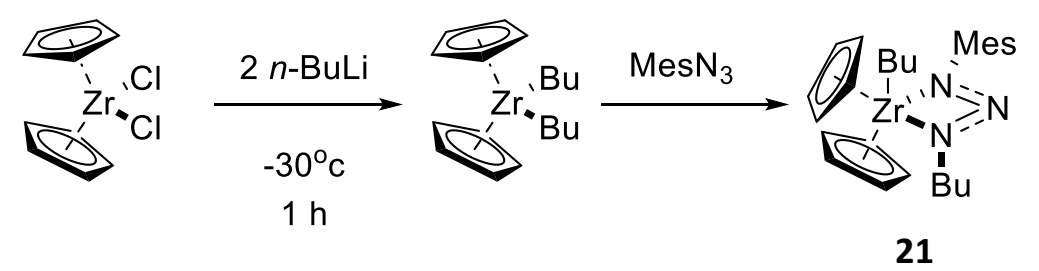

Scheme 29: Synthesis of triazenido complex 21.

The ${ }^{1} \mathrm{H}$ NMR spectrum of $\mathbf{2 1}$ shows the chemical shift downfield $(\delta 3.26,2 \mathrm{H})$ of the triplet signal of one of the two $n$-Bu fragments. This resonance is attributed to the protons on the $\alpha$-carbon of the butyl fragment of the triazenido ligand, which would be expected to have increased deshielding due to the adjacent nitrogen atom. The resonances associated with the mesityl group are shifted downfield $(\delta 6.81(2 \mathrm{H}), 2.33$ $(6 \mathrm{H}), 2.15(3 \mathrm{H}))$ relative to the free mesityl azide $(\delta 6.54(2 \mathrm{H}), 2.13(6 \mathrm{H}), 2.01(3 \mathrm{H}))$, indicating a change to a more deshielded chemical environment. The presence of several signals in the high field of the spectrum indicates that there are two $n$-Bu fragments present in $\mathbf{2 1}$, indicating that the $\beta$-hydrogen elimination of the dialkyl zirconocene to give the Negishi reagent never occurred, and instead the azide inserted into one of two zirconium alkyl fragments present.

Crystals suitable for an X-ray diffraction study were obtained from a concentrated toluene solution of 21. The X-ray crystal structure data of $\mathbf{2 1}$ shows 2 molecules in the asymmetric unit with approximately the same bond lengths and angles, hence only one molecule will be discussed here. The 5-coordinate zirconium metal centre is in a distorted trigonal bipyramidal geometry (Figure 47). The two alkyl fragments are oriented anti to each other, and the plane of the mesityl group is orientated almost orthogonal to the equatorial $\mathrm{ZrN}_{3}$ plane, presumably to minimize steric repulsion. Comparing the structure to the most similar complex in the literature, XXXIV; the Zr-N distances in $\mathbf{2 1}$ are extremely similar (2.339 and 2.314 $\AA$ )), indicating the delocalisation of electrons over the all three of the nitrogens. This differs from complexes XXXIV, which has one of the nitrogen-nitrogen bonds almost $0.2 \AA$ A shorter (2.388 compared to $2.197 \AA$ ) , though this compound does not have delocalisation of electron density over all three nitrogen atoms, so a different bond length would be expected. ${ }^{55}$ The bite angle of the triazenido ligand (53.63 $\AA$ ) is comparable to the literature values for triazenido ligands on zirconium (IV). ${ }^{122}$ The two butyl groups are orientated anti each other $\left(C(1)-\left(\operatorname{Zr}(1)-N(3)-C(14)\right.\right.$ dihedral angle $\left.(D H A)=178.13^{\circ}\right)$. 

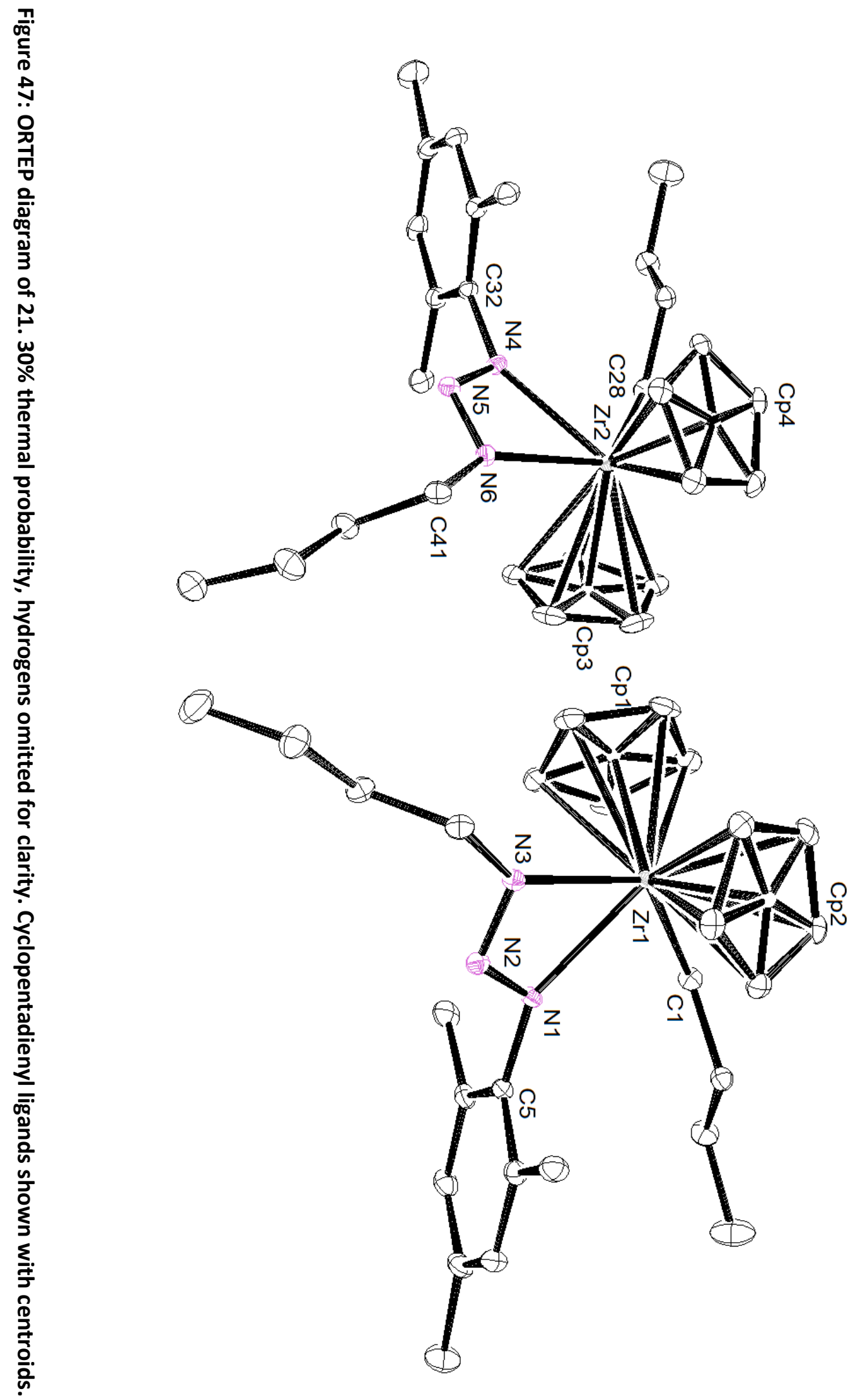
Table 24: Selected bond lengths and angles of 21.

\section{Bond Lengths (Å)}

$\begin{array}{cc}\operatorname{Zr}(1)-\mathrm{N}(1) & 2.339(2) \\ \operatorname{Zr}(1)-\mathrm{N}(3) & 2.314(2) \\ \operatorname{Zr}(1)-\mathrm{C}(1) & 2.325(2) \\ \mathrm{N}(3)-\mathrm{C}(14) & 1.464(3) \\ \mathrm{N}(1)-\mathrm{C}(5) & 1.437(3) \\ \operatorname{Zr}(1)-\mathrm{Cp}(1) & 2.2251(11) \\ \operatorname{Zr}(1)-\mathrm{Cp}(2) & 2.2460(13)\end{array}$

Bond Angles ( $\left.{ }^{\circ}\right)$

$\begin{array}{cc}N(1)-N(2)-N(3) & 107.0(2) \\ N(1)-\operatorname{Zr}(1)-N(3) & 53.63(7) \\ \operatorname{Zr}(1)-N(1)-C(5) & 147.41(16) \\ \operatorname{Zr}(1)-N(3)-C(14) & 145.17(17) \\ C p(1)-\operatorname{Zr}(1)-C p(2) & 127.90(5) \\ (N(1)-\operatorname{Zr}(1)-C(1) & 77.85(8)\end{array}$

\subsubsection{Synthesis of $\left[\mathrm{Cp}_{2} \mathrm{Zr}(\mathrm{Me})\left(\mathrm{N}_{3}(\mathrm{Me})(\mathrm{Mes})\right)\right] \quad 22$}

Having synthesised $\mathbf{2 1}$ it was decided that the other readily accessed bis-alkyl zirconocenes would be synthesised to gain some insight as to the effect of the alkyl fragment on the structure and bonding of the triazenido zirconocene complex. The reaction of $\left[\mathrm{Cp}_{2} \mathrm{ZrMe}_{2}\right]$ with one equivalent of $\left[\mathrm{MesN}_{3}\right]$ in toluene generated the insertion product with high yield (85\%).

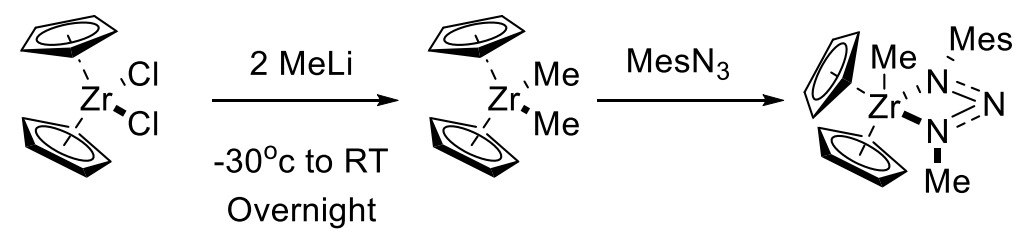

22

Scheme 30: Synthesis of 22 via bismethylzirconocene.

The ${ }^{1} \mathrm{H}$ NMR spectrum of 22 shows the downfield chemical shift to $\delta 3.00(3 \mathrm{H})$ of one of the methyl singlets, which is attributed to the methyl going from the extremely shielded zirconium methyl present in $\left[\mathrm{Cp}_{2} \mathrm{ZrMe}_{2}\right](\delta-0.12)$ to the more deshielded triazenido ligand due to the electronegative nitrogen atom. A second methyl environment is observed at $\delta-0.23(3 \mathrm{H})$. The chemical shift indicates increased shielding relative to the starting material. The methyl mesityl resonances are also downfield shifted relative to the azide starting material to $\delta 2.33(6 \mathrm{H})$ and $2.17 \mathrm{ppm}(3 \mathrm{H})$. The meta protons of the mesityl group resonate as a singlet at $\delta 6.82 \mathrm{ppm}(2 \mathrm{H})$, while the cyclopentadienyl resonance is a singlet at $\delta 5.62 \mathrm{ppm}$.

Crystals suitable for an X-ray diffraction study were obtained from a concentrated diethyl ether solution of 22. The solid-state crystal structure data reveals $\mathbf{2 2}$ has one molecule in the asymmetric unit. The 
molecule has the same conformation as $\mathbf{2 1}$, with the mesityl ring almost orthogonal to the plane on the three nitrogen atoms (the angle between planes $\left.95.9(6)^{\circ}\right)$. As per 21, the crystal structure data for 22 reveals the two alkyl fragments are orientated anti to each other $\left(C(1)-N(3)-\operatorname{Zr}(1)-C(2) D H A=169.45^{\circ}\right)$. This DHA is smaller than 21, possibly due to the reduced steric bulk of the methyl ligand relative to the butyl ligand. The bite angle of the triazenido ligand in 20 is $53.7(2)^{\circ}$, effectively the same as $\mathbf{2 1}\left(53.63(7)^{\circ}\right)$.

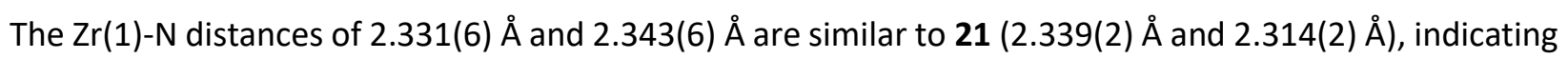
the change of alkyl substituent has little effect on the coordination of the triazenido ligand. $\operatorname{The} \operatorname{Zr}(1)-C(2)$ distance of 2.297(9) $\AA$ is shorter than the $\operatorname{Zr}(1)-C(1)$ distance of 21 (2.325(2) $\AA$ ). Other bond distances and angles are available in Table 25.

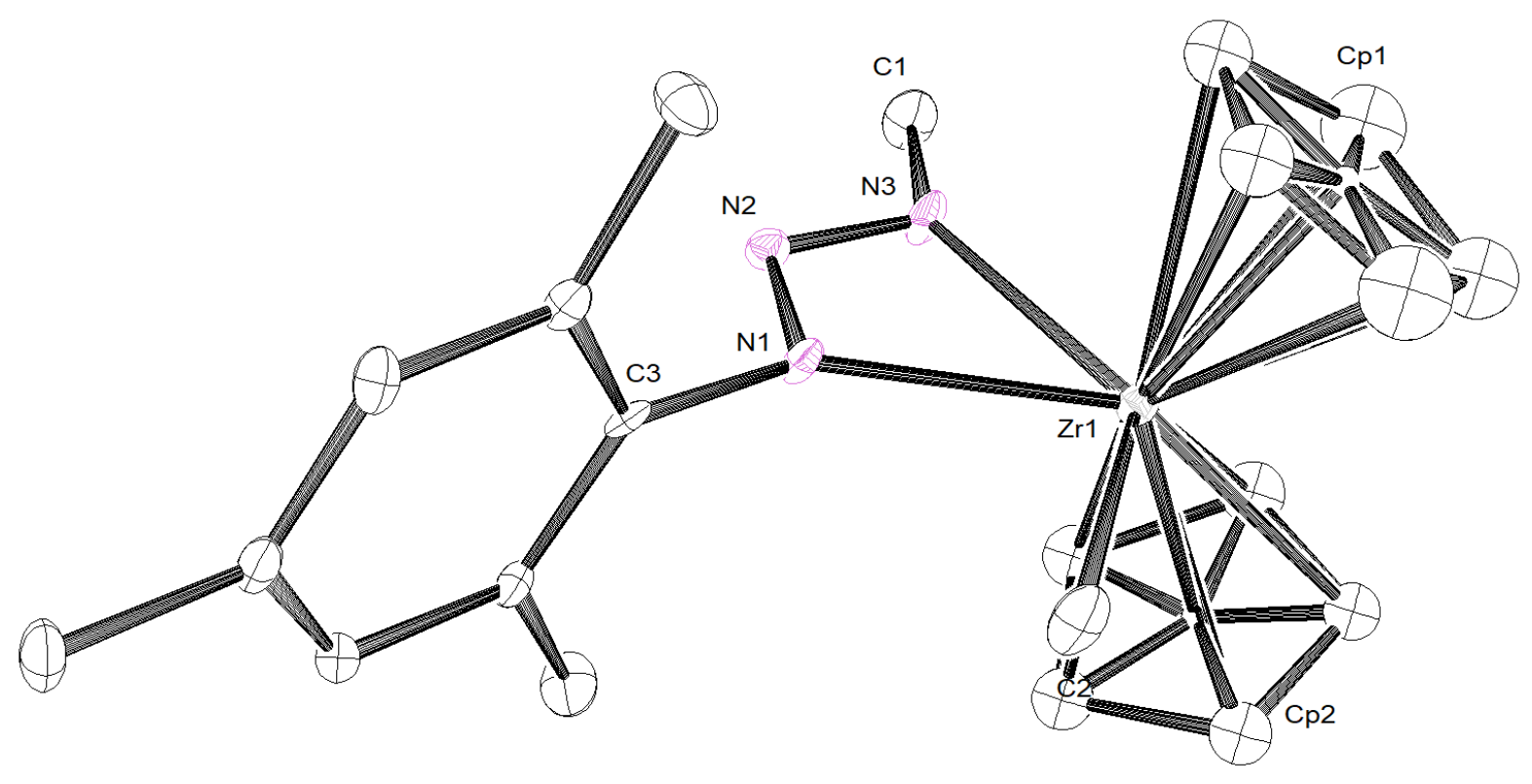

Figure 48: ORTEP diagram of $22.30 \%$ thermal probability, hydrogens omitted for clarity. Cyclopentadienyl ligands shown with centroids.

Table 25: Selected bond lengths and angles of 22.

Bond Lengths (Å)

Bond Angles ( $\left.{ }^{\circ}\right)$

$\begin{array}{lc}\operatorname{Zr}(1)-\mathrm{N}(3) & 2.331(6) \\ \operatorname{Zr}(1)-\mathrm{N}(1) & 2.343(6) \\ \operatorname{Zr}(1)-\mathrm{C}(2) & 2.297(9) \\ \mathrm{N}(3)-\mathrm{C}(1) & 1.44(1) \\ \mathrm{N}(2)-\mathrm{N}(1) & 1.333(8)\end{array}$

$\begin{array}{lc}\mathrm{N}(1)-\mathrm{Zr}(1)-\mathrm{N}(3) & 53.7(2) \\ \mathrm{N}(3)-\mathrm{Zr}(1)-\mathrm{C}(2) & 128.4(3) \\ \mathrm{Zr}(1)-\mathrm{N}(1)-\mathrm{C}(3) & 144.1(6) \\ \mathrm{Zr}(1)-\mathrm{N}(3)-\mathrm{C}(1) & 145.9(5) \\ \mathrm{N}(1)-\mathrm{N}(2)-\mathrm{N}(3) & 107.4(6)\end{array}$




$\begin{array}{cccc}\operatorname{Zr}(1)-C p(1) & 2.230(5) & C p(1)-\operatorname{Zr}(1)-C p(2) & 130.4(2) \\ \operatorname{Zr}(1)-C p(2) & 2.225(5) & C p(1)-\operatorname{Zr}(1)-C(2) & 99.6(3) \\ N(1)-C(3) & 1.416(10) & C p(2)-\operatorname{Zr}(1)-C(2) & 98.2(3)\end{array}$

\subsubsection{Synthesis of $\left[\mathrm{Cp}_{2} \mathrm{Zr}(\mathrm{Me})\left(\mathrm{N}_{3}(\mathrm{Me})\left(\mathrm{C}_{6} \mathrm{H}_{5}\right)\right)\right] 23$}

Compound $\mathbf{2 3}$ was synthesised from the addition mesitylazide to $\left[\mathrm{Cp}_{2} \mathrm{ZrMe}_{2}\right]$ at $-30^{\circ} \mathrm{C}$ in diethyl ether. 23 was characterised by ${ }^{1} \mathrm{H}$ NMR spectroscopy, ${ }^{13} \mathrm{C}$ NMR spectroscopy, IR, elemental analysis and single crystal X-ray diffraction.

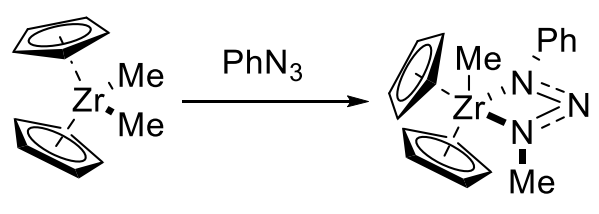

23

The ${ }^{1} \mathrm{H}$ NMR spectrum shows the characteristic downfield shift for the NMe environment at $\delta 3.14 \mathrm{ppm}$ $(3 \mathrm{H})$, likely due to the increased deshielding from the $\alpha$ nitrogen atom. The zirconium bonded methyl environment is chemical shifted downfield to $\delta 0.04 \mathrm{ppm}(3 \mathrm{H})$ from $\delta-0.12 \mathrm{ppm}$ and the cyclopentadienyl resonance is upfield shifted to $\delta 5.60 \mathrm{ppm}(1 \mathrm{OH})$ from the starting material $(\delta 5.71 \mathrm{ppm})$. The aromatic signals are observed as two multiplet at $\delta 7.33-7.28 \mathrm{ppm}(4 \mathrm{H})$ for the ortho and meta protons and $\delta 7.01$ ppm (1H) for the para substituted proton.

Crystals suitable for an X-ray diffraction study were obtained from a concentrated diethyl ether solution of 23. The solid-state crystal structure of $\mathbf{2 3}$ has $\mathbf{2}$ molecules of in the asymmetric unit. These molecules have extremely similar bond lengths and angles, as such only one molecule will be discussed in detail. There are 5 coordinated sites occupied resulting in a distorted trigonal pyramidal geometry around the central zirconium atoms with the $Z r$-methyl ligand and triazenido ligand occupying the equatorial plane. Unlike 22 the methyl groups are located syn to each other $C(1), N(3)-\operatorname{Zr}(2)-C(4) D H A=2.99^{\circ}$. As 22 has the anti-DHA of the methyl ligands, it is indicated that the steric bulk of the azide the determines the syn or anti orientation of the alkyl groups. The zirconium nitrogen bond, $\operatorname{Zr}(1)-N(5),(2.356(3) \AA)$ in which the nitrogen has an $\alpha$ phenyl substituent of is longer than the zirconium nitrogen bond length with an $\alpha$ methyl substituent; $\operatorname{Zr}(1)-\mathrm{N}(4)$ of $2.296(3) \AA$, reflecting the non-symmetrical nature of the triazenido ligand. The 
zirconium cyclopentadienyl bond distances are shorter than in $\mathbf{2 2}$, though whether this is a steric or electronic effect is unknown. The angle between the cyclopentadienyl planes is similar to 22.
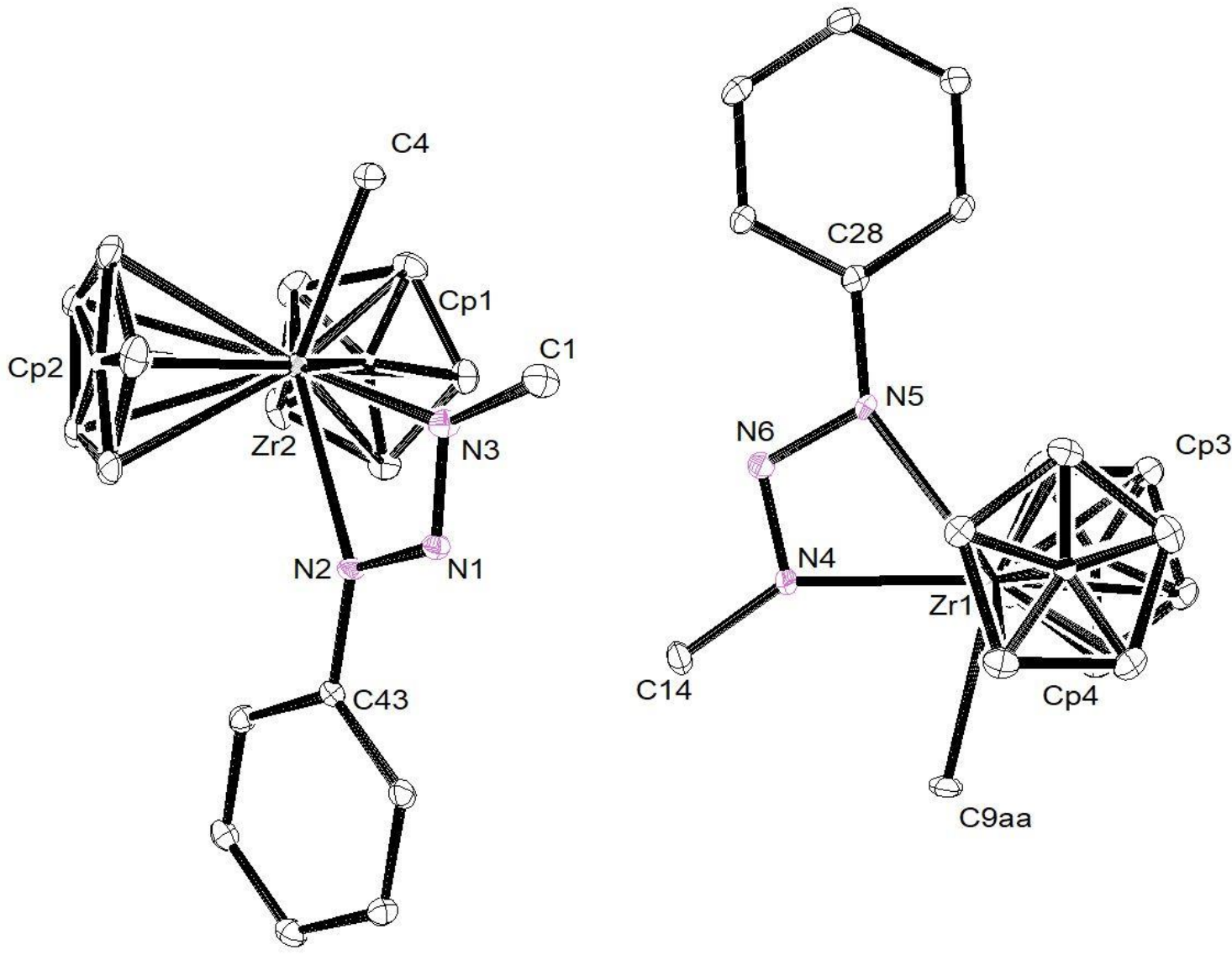

Figure 49: ORTEP representation of 23. 30\% Thermal ellipsoids. Hydrogen atoms omitted for clarity.

Table 26: Selected bond lengths and angles of 23.

\begin{tabular}{lc}
\multicolumn{2}{c}{ Bond Lengths (Å) } \\
$\operatorname{Zr}(1)-\mathrm{Cp}(3)$ & $2.2233(15)$ \\
$\mathrm{Zr}(1)-\mathrm{Cp}(4)$ & $2.2244(16)$ \\
$\mathrm{N}(4)-\mathrm{N}(6)$ & $1.303(4)$ \\
$\mathrm{N}(6)-\mathrm{N}(5)$ & $1.319(4)$ \\
$\mathrm{Zr}(1)-\mathrm{C}(9)$ & $2.330(3)$ \\
$\mathrm{Zr}(1)-\mathrm{N}(5)$ & $2.356(3)$ \\
$\operatorname{Zr}(1)-\mathrm{N}(4)$ & $2.296(3)$
\end{tabular}

Bond Angles ( $\left.{ }^{\circ}\right)$

$\begin{array}{cc}\mathrm{Cp}(1)-\mathrm{Cp}(2) \text { planes } & 129.17(15) \\ \mathrm{N}(4)-\mathrm{Zr}(1)-\mathrm{N}(5) & 53.84(10) \\ \mathrm{Zr}(1)-\mathrm{N}(4)-\mathrm{C}(14) & 145.2(2) \\ \mathrm{Zr}(1)-\mathrm{N}(6)-\mathrm{C}(28) & 146.9(2) \\ \mathrm{Cp}(3)-\mathrm{Cp}(4) \text { planes } & 129.52(7) \\ \mathrm{N}(4)-\mathrm{N}(5)-\mathrm{N}(6) & 107.0(3)\end{array}$




\subsubsection{Synthesis of $\left[\mathrm{Cp}_{2} \mathrm{Zr}(\mathrm{Bn})\left(\mathrm{N}_{3}(\mathrm{Bn})(\mathrm{Mes})\right)\right]$}

24 was synthesised from the treatment of $\left[\mathrm{Cp}_{2} \mathrm{ZrBn}_{2}\right]$ with mesitylazide at $-30{ }^{\circ} \mathrm{C}$. Following workup and purification by crystallisation the compound was characterised by ${ }^{1} \mathrm{H} N M R$ spectroscopy, ${ }^{13} \mathrm{C}$ NMR spectroscopy and single crystal X-ray diffraction.

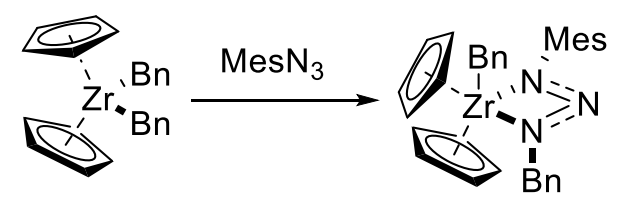

24

The ${ }^{1} \mathrm{H}$ NMR spectrum of 24 shows the significant chemical shift downfield from $\delta 1.83 \mathrm{ppm}$ to $\delta 4.43 \mathrm{ppm}$ $(2 \mathrm{H})$ for the methylene environment of the $\mathrm{ZrBn}$ group that the azide had inserted into. The other methylene $\mathrm{CH}_{2}$ resonance has shifted upfield slightly to $\delta 1.70 \mathrm{ppm}(2 \mathrm{H})$. The phenyl aromatic resonances are visible as 5 signals at $\delta 7.25(2 \mathrm{H}), 7.13(4 \mathrm{H}), 7.09(1 \mathrm{H}), 6.99(2 \mathrm{H}), 6.85 \mathrm{ppm}(1 \mathrm{H})$ for 6 environments, while the meta-substituted phenyl protons that are part of the mesityl group resonate as a singlet at $\delta$ $6.80 \mathrm{ppm}(2 \mathrm{H})$, similar to $22(\delta 6.82 \mathrm{ppm}(2 \mathrm{H}))$. The equivalent cyclopentadienyl ligands resonate at $\delta 5.62$ ppm (10H), upfield from 21 ( $\delta 5.79$ ppm) and 22 ( $\delta 5.69$ ppm), but downfield from 23 ( $\delta 5.60 \mathrm{ppm})$. The mesityl methyl environments are visible in a 2:1 ratio at $\delta 2.33 \mathrm{ppm}(6 \mathrm{H})$ and $\delta 2.14 \mathrm{ppm}(2 \mathrm{H})$, downfield shifted from the free azide.

Crystals suitable for an X-ray diffraction study were obtained from a concentrated hexane solution of 24. The solid-state crystal structure data indicates that $\mathbf{2 4}$ has one molecule present in the asymmetric unit. As per the other triazenido compounds the zirconium atom is 5 coordinate at the zirconium atom with a distorted trigonal bipyramidal geometry. The triazenido ligand and $\mathrm{ZrBn}$ ligand occupy the equatorial plane, while the of the cyclopentadienyl ligands (as measured from the centroid in the centre of each ring) are separated by an angle of $128.26(6)^{\circ}$. The bite angle $(\mathrm{N}(1)-\operatorname{Zr}(1)-\mathrm{N}(3))$ of the triazenido ligand is $53.72^{\circ}$, similar to compounds $\mathbf{2 1}\left(53.63^{\circ}\right), \mathbf{2 2}\left(52.7^{\circ}\right)$ and $\mathbf{2 3}\left(53.84^{\circ}\right)$, indicating that the bite angle doesn't appear to the changing electronic and steric properties of the substituents. $\operatorname{The} \operatorname{Zr}(1)-C(17)$ bond distance of $2.384(2) \AA ̊$ is slightly longer than the zirconium carbon bond of $\mathbf{2 1}(2.325(2) \AA)$ and $\mathbf{2 2}$ (2.330(3) $\AA$ ), though whether this is an electronic effect through increased $\sigma$-donation or a steric effect was unable to be determined. The benzyl groups are orientated anti to one another $(C(10)-N(1)-\operatorname{Zr}(1)-C(17) D H A=172.84)$, 
as would be expected if the observation that the orientation is dependent on the steric bulk of the azide is correct.

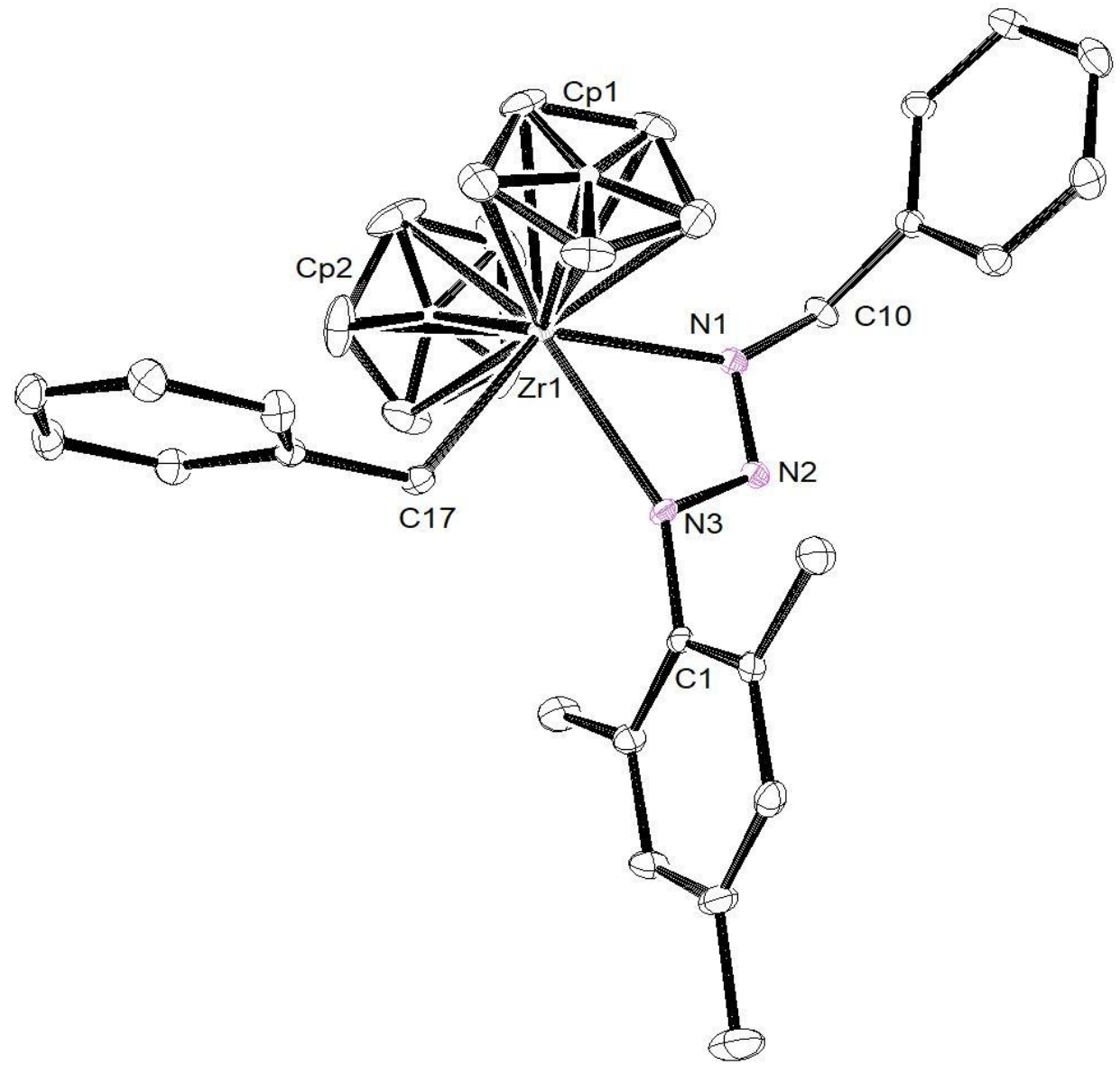

Figure 50: ORTEP diagram of $\mathbf{2 4}$ with $\mathbf{3 0 \%}$ thermal probability, hydrogens omitted for clarity. Cyclopentadienyl ligands shown with centroids.

Table 27: Selected bond lengths and angles of 24.

Bond Lengths (Å)

$\begin{array}{cc}\operatorname{Zr}(1)-\mathrm{N}(3) & 2.329(2) \\ \operatorname{Zr}(1)-\mathrm{N}(1) & 2.342(2) \\ \operatorname{Zr}(1)-\mathrm{C}(17) & 2.384(2) \\ \mathrm{N}(2)-\mathrm{N}(1) & 1.294(3) \\ \operatorname{Zr}(1)-\mathrm{Cp}(1) & 2.2193(12) \\ \operatorname{Zr}(1)-\mathrm{Cp}(2) & 2.2305(13)\end{array}$

Bond Angles ( ${ }^{\circ}$ )

$\begin{array}{lc}N(1)-\operatorname{Zr}(1)-N(3) & 53.72(7) \\ N(3)-\operatorname{Zr}(1)-C(17) & 74.24(8) \\ \operatorname{Zr}(1)-N(3)-C(6) & 147.87(6) \\ \operatorname{Zr}(1)-N(1)-C(1) & 146.26(16) \\ N(1)-N(2)-N(3) & 107.76(18) \\ C p(1)-\operatorname{Zr}(1)-C p(2) & 128.26(6) \\ C p(1)-\operatorname{Zr}(1)-C(17) & 102.08(7) \\ C p(2)-\operatorname{Zr}(1)-C(17) & 102.35(7)\end{array}$




\subsection{Summary of Results}

The novel amidozirconocenes 14, 15, 16, 17, 18 and 19 were synthesised for their use in the synthesis of 1. The amidozirconocenes have varying steric bulk on the amide ligand so that the effect of amide bulk on the synthesis of 1 can be investigated. The amides 14 ([C $\left.\left.\mathrm{Cp}_{2} \mathrm{Zr}(\mathrm{NHDipp})(\mathrm{Me})\right]\right), 15\left(\left[\mathrm{Cp} \mathrm{Zr}_{2}(\mathrm{Me})(\mathrm{NHAr} *)\right]\right)$ and $16\left(\left[\mathrm{Cp}_{2} \mathrm{Zr}(\mathrm{Me})(\mathrm{NH}(\mathrm{DMP}))\right]\right)$ indicated that as the steric bulk of the amide is increased, the $\mathrm{Zr}-\mathrm{N}-\mathrm{C}$ angle of

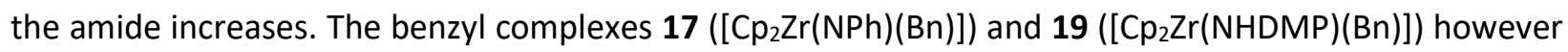
indicate the opposite effect, with the $\mathrm{Zr}-\mathrm{N}-\mathrm{C}$ bond angle decreasing with increased steric bulk. Hence more information is needed in order to fully understand the relationship. The imidozirconocene $\mathbf{2 0}$ was synthesised from the thermolysis of $\mathbf{1 5}$ with THF, and crystal structure data showed similar bond lengths and angles to the literature complexes $\mathbf{X X X}$ and $\mathbf{X X X I}(\mathbf{a})$. The insertion reactions of mesitylazide and phenyl azide into $\left[\mathrm{Cp}_{2} \mathrm{ZrMe} \mathrm{r}_{2}\right],\left[\mathrm{Cp}_{2} \mathrm{ZrBn}_{2}\right]$ and $\left[\mathrm{Cp}_{2} \mathrm{ZrBu}_{2}\right]$ were investigated and it was determined that the steric bulk of the azide influences the structural configuration of the triazenido product. The synthesis of the butyl complex $\mathbf{2 1}$ indicated that the Negishi reagent was not generated and instead the azide moeity were inserting into the bis-alkylzirconocene. 


\section{Results and Discussion: Group 4 - Group 14 Heterobimetallics}

\subsection{Synthetic Strategy}

The strategy employed towards synthesising an amido-bridged germanium zirconium complex was based on two main approaches. The first was the reaction of the imidozirconocene with a germylene, while the second was the reaction of reduced zirconium(II) with a germanimine. Since the reaction of imidozirconocene with a germylene was thought to be the more desirable synthetic route, the majority of reactions that were carried out used the attempted route of an imidozirconocene with a germylene, which was thought would either act as a donor to stabilise the zirconium nitrogen bond, or rearrange to provide the desired heterobimetallic.

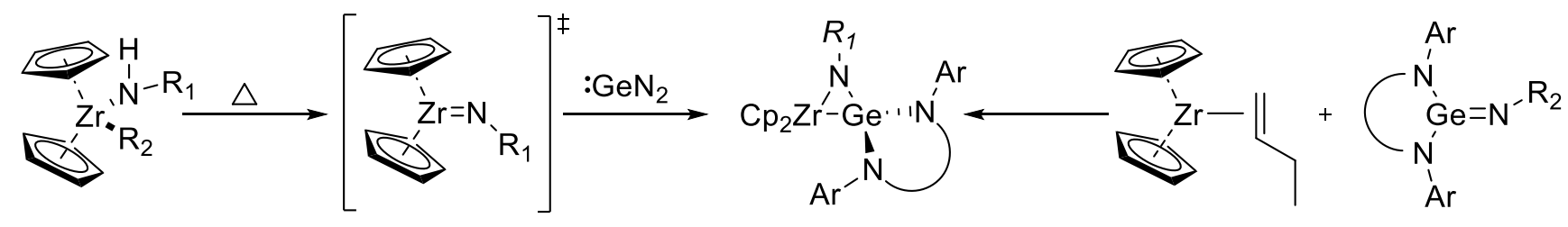

Scheme 31: Proposed Synthetic Route to heterobimetallic 1.

\subsection{Reactions between Group 4 and Group 14 Compounds}

\subsubsection{Addition of $\left[\mathrm{Cp}_{2} \mathrm{ZrMe}_{2}\right]$ to [DippNH $\left.\mathrm{H}_{2}\right]$ in the presence of [Ge(NAPH $\left.\left.{ }^{\mathrm{TMS}}\right)\right]$}

The reaction of bismethylzirconocene with diisopropylaniline and [Ge( $\left.\left.\mathrm{NAPH}^{\mathrm{TMS}}\right)\right]$ was carried out in an NMR tube fitted with a young's tap and heated on an aluminium heating block to $75^{\circ} \mathrm{C}$. Reaction progress was monitored by ${ }^{1} \mathrm{H}$ NMR spectroscopy. After 2 days of heating the ${ }^{1} \mathrm{H}$ NMR resonances attributed to the zirconium amide could be seen growing in along with another set of diisopropyl peaks. Further heating resulted in a number of new resonances indicating a range of new products. The ${ }^{1} \mathrm{H}$ NMR spectrum did not give an indication of the desired reaction product, as although there was a new intense peak growing in, consistent with a isopropyl environment, there was no observed change to the TMS resonances of 
[Ge(NAPH $\left.\left.{ }^{\mathrm{TMS}}\right)\right]$. The low field zirconium alkyl resonance did also not disappear to give methane as desired. Although attempted, separation of the products was not successful.

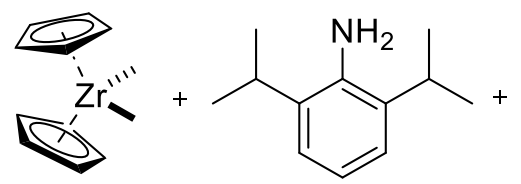<smiles>CN(C)c1cccc2cccc(N(C)S(C)(=O)=O)c12</smiles>
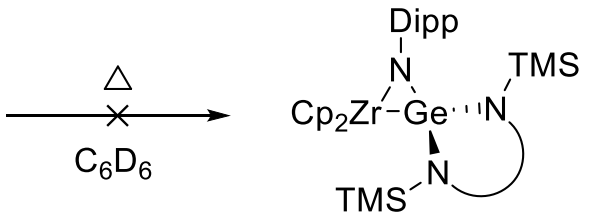

\subsubsection{Addition of $\left[\mathrm{Cp}_{2} \mathrm{Zr}(\mathrm{Me})(\mathrm{NHDipp})\right]$ to [Ge(NAPH $\left.\left.{ }^{\mathrm{TMS}}\right)\right]$}

To investigate whether the THF stabilized imidozirconocene would react with [Ge(NAPH $\left.{ }^{\mathrm{TMS}}\right)$ ], giving an imido-bridged heterobimetallic, or germylene stabilized imidozirconocene, the addition of 2 equivalents of THF to $\left[\mathrm{Cp}_{2} \mathrm{Zr}(\mathrm{Me})(\mathrm{NHDipp})\right]$ followed by heating formed the imidozirconocene in situ. Presence of the imidozirconocene was confirmed from comparison to literature ${ }^{1} \mathrm{H}$ NMR spectroscopy values. ${ }^{54}$ To this solution was added [Ge( $\left.\left.\mathrm{NAPH}^{\mathrm{TMS}}\right)\right]$ and ${ }^{1} \mathrm{H}$ NMR spectra were taken after various temperature intervals.

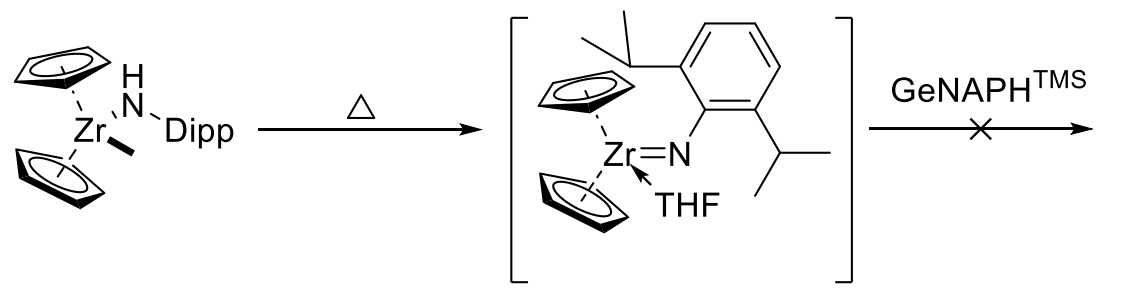

14

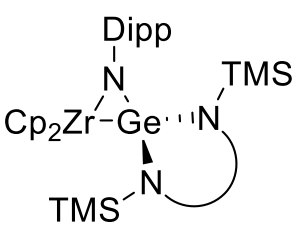

1

Scheme 32: Proposed reaction to amide bridged heterobimetallic 1.

Analysis of the ${ }^{1} \mathrm{H}$ NMR spectra of the crude reaction mixture indicated that there was no change in chemical environment of the germylene or imidozirconocene (no change to a chemical shift or change in resonance multiplicity). The imidozirconocene species appeared to decompose after several days, with a range of new cyclopentadienyl peaks in the region $\delta 6.4-5.3$ ppm while the characteristic isopropyl resonances at $\delta 3.70$ (septet) and 1.38 (doublet) ppm slowly diminished. Although no single product could be isolated, the chemical shifts in the ${ }^{1} \mathrm{H}$ NMR spectra indicated the imidozirconocene [Cp $\left.2 \mathrm{Zr}(\mathrm{NDipp})(\mathrm{THF})\right]$ had been synthesised before subsequently decomposing. Although not a success, this indicates that that the THF stabilised imidozirconocene was more stable than the proposed germylene stabilised imidozirconocene. This is perhaps unsurprising, as THF is known to be a strong donor and coordinating 
solvent. Although exchange of the THF molecule for the germylene was expected, the ${ }^{1} \mathrm{H}$ NMR spectra indicated that the lone pair on complex $\mathbf{3}$ is not a strong enough donor atom to displace THF.

\subsubsection{Addition of $\left[\mathrm{Cp}_{2} \mathrm{Zr}(\mathrm{NAr} *)(\mathrm{THF})\right]$ to $\left[\mathrm{Ge}\left(\mathrm{BIAN}^{\mathrm{Mes}}\right)\right]$}

To investigate whether the THF stabilised imidozirconocenes were reactive towards a different germylene, a reaction similar to that above was carried out. To one equivalent of the bulky isolated imidozirconocene $\mathbf{2 0}$ was added one equivalent of the germylene [GeBIAN ${ }^{\text {Mes}}$. The reaction was carried out on an NMR scale so that the reaction could be monitored throughout by ${ }^{1} \mathrm{H}$ NMR spectroscopy.

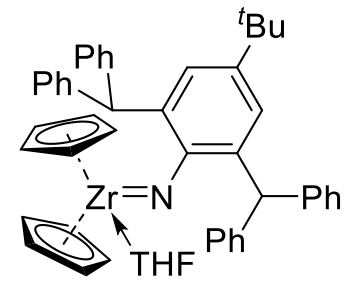

20

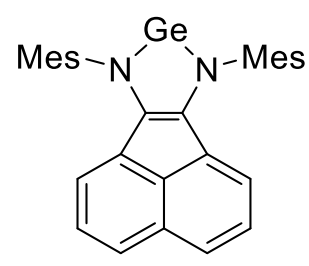

2

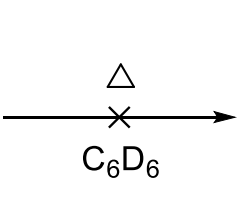

.

(20)

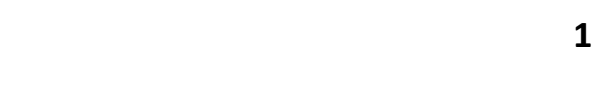

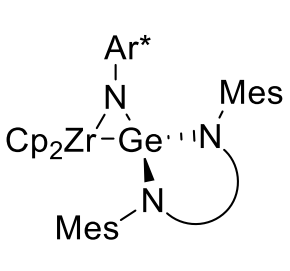

1

The ${ }^{1} \mathrm{H}$ NMR spectrum indicated no change to the [GeBIAN ${ }^{\text {Mes }}$ ] resonances for the aromatic BIAN backbone at $\delta 7.20$ (doublet), 6.90 (triplet), and 6.59 (doublet) ppm, or to the mesityl resonances at $\delta 6.94$ (singlet), 2.29 (singlet) or 2.26 (singlet), indicating no change in environment in solution for any of the atoms present in [GeBIAN ${ }^{\text {Mes }}$. A new singlet at $\delta 1.23 \mathrm{ppm}$ grew in over 5 days at $100^{\circ} \mathrm{C}$, this was assumed to be a new tert-butyl environment as the resonance of the tert-butyl peak of 18 ( $\delta 1.17 \mathrm{ppm})$ decreased proportionally. Although this indicated the presence of one new product, a number of new resonances in the cyclopentadienyl region of chemical shifts $(\delta 5.59,5.58$ and $5.48 \mathrm{ppm})$ indicated a range of cyclopentadienyl environments, either indicating a number of products or possible loss of symmetry of the cyclopentadienyl ligands. Due to the high number of aromatic protons present in $\mathbf{1 8}$ the aromatic region a complex series of multiplets, and hence did not help identify the species present in solution. Attempts to isolate individual products proved unsuccessful, however it seems likely that the imidozirconocene was undergoing some sort of decomposition at $100{ }^{\circ} \mathrm{C}$ and did not proceed to the desired heterobimetallic complex. 


\subsubsection{Addition of $\left[\mathrm{Cp}_{2} \mathrm{Zr}\left(\mathrm{NHAr}^{*}\right)(\mathrm{Me})\right]$ to $\left[\mathrm{GeBIAN}^{\mathrm{Mes}}\right]$}

Since substitution of the relative donor molecules didn't occur from the treatment of the $\mathbf{1 8}$ with the germylenes $\mathbf{2}$ or $\mathbf{3}$, thermal elimination by the alkyl-amidozirconocene in the presence of a germylene was attempted (eq. 31). Prior reactions had indicated that without the presence of THF present the thermal elimination of methane would not proceed to the imidozirconocene, hence the reaction of $\mathbf{1 5}$ with $\mathbf{2}$ was carried out to investigate whether the germylene would act as a donor molecule when a more suitable donor molecule such as THF was not present.

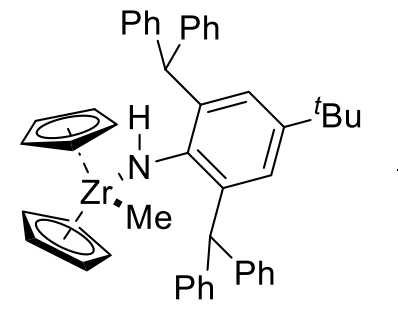

15

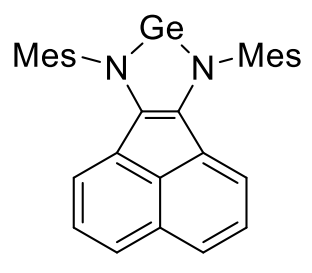

2

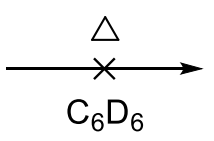

1

The reaction was carried out in an NMR tube fitted with a Young's tap in order to monitor the reaction by ${ }^{1} \mathrm{H}$ NMR spectroscopy. Over the period of 5 days up to $100{ }^{\circ} \mathrm{C}$ the methyl peak of 15 vanished, indicating the elimination of methane, which was confirmed from the increased intensity of a new signal attributed to methane at $0.16 \mathrm{ppm}^{71}$ The tert-butyl singlet resonance at $\delta 1.10 \mathrm{ppm}$ became weaker over the 5 days while a new singlet at $\delta 1.24 \mathrm{ppm}$ grew in proportion to the diminishing signal, indicating the presence of a single new tert-butyl environment (likely the same product as the reaction described by eq. 30 ). The cyclopentadienyl singlet of the amide $(\delta 5.59 \mathrm{ppm})$ diminishes over the 5 days, with two new singlets appearing in spectrum at $\delta 5.60$ and $5.49 \mathrm{ppm}$ respectively attributed to two new cyclopentadienyl environments. There was no observed change to the [Ge(BIAN $\left.\left.{ }^{M e s}\right)\right]$ aromatic signals of $\delta 7.20$ (doublet), 6.90 (triplet), and 6.59 (doublet) ppm, or to the mesityl resonances at 6.94 (singlet), 2.29 (singlet) or 2.26 (singlet), indicating no coordination or decomposition of the germylene over the 5 days. Interestingly, the signals at $\delta 5.49$, and $1.24 \mathrm{ppm}$ indicate that the reaction of the amide 15 with 2 and the reaction of the imidozirconocene $\mathbf{2 0}$ with $\mathbf{2}$ may go to the same product. This perhaps shouldn't be surprising as it is likely that the imidozirconocene is being generated in situ. However, there is no evidence of the imidozirconocene in the ${ }^{1} \mathrm{H}$ NMR spectrum, indicating that if the imidozirconocene is being generated, it is then reacting again instantly. This is in contrast to the THF stabilised imidozirconocene, which was observed to react over several days, even at $100^{\circ} \mathrm{C}$. 


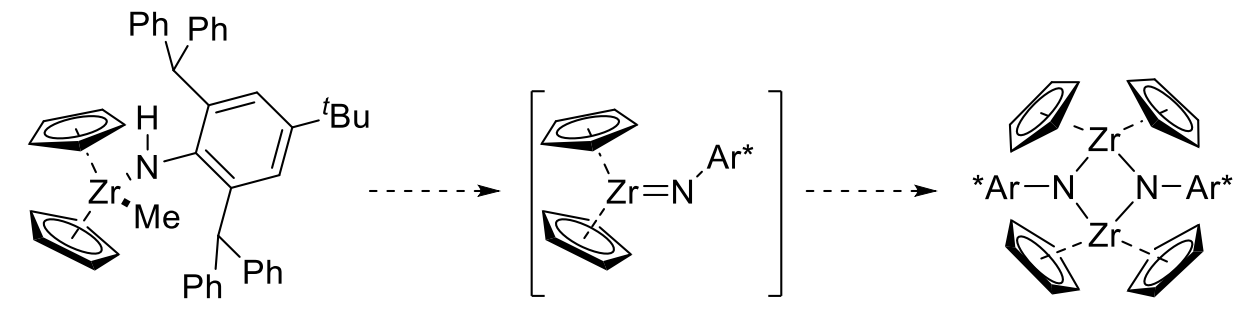

15

Scheme 33: Possible reaction of 15 to zirconium tetracycle.

Assuming generation of the imidozirconocene in situ, it seems most likely that the reaction product could be the [2+2] cycloaddition dimer (zirconium tetracycle) (Scheme 33). Complexes of this type are known (including $\mathbf{X X X I ( d ) ~ a n d ~ 1 8 ) , ~ a l t h o u g h ~ t h e ~ z i r c o n i u m ~ a t o m s ~ h a v e ~ p r e v i o u s l y ~ o n l y ~ b e e n ~ r e p o r t e d ~ s u p p o r t e d ~}$ by the dimethyl amide ligand with bridging amidosilyl or amidotertbutyl ligands, or with the relatively small 4-tert-butylphenyl amide supported with cyclopentadienyl ligands. ${ }^{49,}{ }^{123-124} \mathbf{1 5}$ was chosen for use in this reaction due to the large steric bulk of [NHAr*], which it was assumed would kinetically hinder the cycloaddition through steric stabilisation. A simple way to investigate Scheme $\mathbf{3 4}$ would be to heat $\mathbf{1 5}$ or 19 to see whether the same ${ }^{1} \mathrm{H}$ NMR resonance are seen in the ${ }^{1} \mathrm{H}$ NMR spectrum.

\subsubsection{Addition of $\left[\mathrm{DMPNH}{ }_{2}\right]$ to $\left[\mathrm{Cp}_{2} \mathrm{ZrBn}_{2}\right]$ with $\left[\mathrm{Ge}(\mathrm{HMDS})_{2}\right]$}

To see whether using a smaller amine source [2-6- $\left.\mathrm{MeC}_{6} \mathrm{H}_{3} \mathrm{NH}_{2}\right]$ and different germylene would promote formation of 1, the reaction detailed in Scheme 34 was carried out. The reagents were heated together to $70{ }^{\circ} \mathrm{C}$ over 5 days in an NMR tube fitted with a Young's tap. ${ }^{1} \mathrm{H}$ NMR spectra were collected over the period to the monitor the reaction in situ. Over this period, a change in the cyclopentadienyl resonance from $\delta 5.51 \mathrm{ppm}$ to two new resonances in an approximate 1:10 ratio at $\delta 5.56$ and $5.44 \mathrm{ppm}$ was observed. The new major product cyclopentadienyl peak at $\delta 5.44 \mathrm{ppm}$ was attributed to the benzyl amide species 19, while the product at $\delta 5.56$ was the suspected zirconium tetracylcle, although this product could not be isolated for further analysis. The presence of a new singlet at $\delta 3.35 \mathrm{ppm}$ which integrated in a 6:10 ratio for the 2,6 methyl positions relative to the new cyclopentadienyl resonance indicates either the synthesis of the imidozirconocene or the zirconium tetracycle shown in Scheme 34. In the high field the $\left[\mathrm{Ge}(\mathrm{HMDS})_{2}\right]$ resonance at $\delta 0.32 \mathrm{ppm}$ fully disappears after the first 24 hours of heating at $70^{\circ} \mathrm{C}$, with a new singlet at $\delta 0.09 \mathrm{ppm}$ appearing with an equivalent integral to the [Ge(HMDS) ${ }_{2}$ ] starting material. This new resonance in likely the protonated free ligand [(HMDS)-H], which is known to appear at this chemical shift. This resonance could either occur through thermal degradation of $\left[\mathrm{Ge}(\mathrm{HMDS})_{2}\right]$, or ligand exchange with the 2,6-dimethylaniline. Thermal degradation requires the ligand to pick up proton, while 
ligand transfer would be expected to give the by-product [Ge(NHDMP $\left.)_{2}\right]$, which was not observed in the ${ }^{1} \mathrm{H}$ NMR spectrum. The low field shows a new triplet at $\delta 7.34 \mathrm{ppm}$, doublet at $\delta 7.22 \mathrm{ppm}$, attributed the new amide resonances, as well as toluene signals at $\delta 7.13$ (multiplet), 7.02 (multiplet) and $2.11 \mathrm{ppm}$ (singlet) indicating the hydride transfer from the free amine to the benzyl group to give toluene as a reaction by-product.

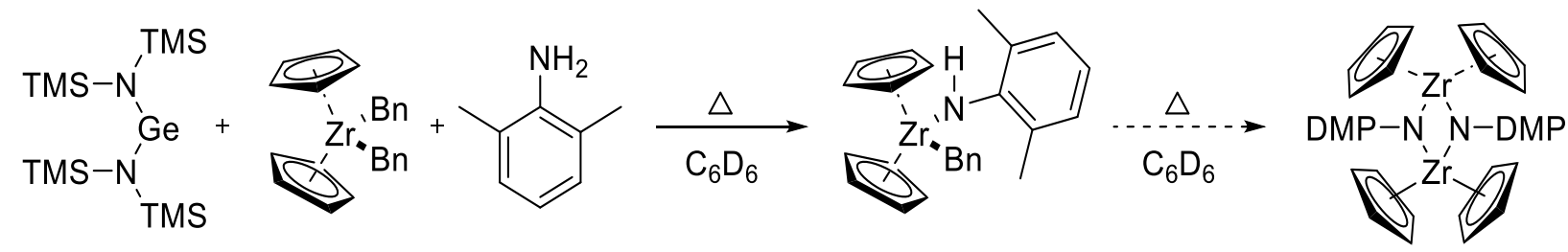

Scheme 34: Reaction of Bisbenzylzirconcene with 2,6-dimethylaniline and [Ge(HMDS) ${ }_{2}$.

\subsubsection{Addition of $\left[\mathrm{Ge}(\mathrm{HMDS})_{2}\right]$ to $\left[\mathrm{Cp}_{2} \mathrm{Zr}(\mathrm{NHPh})(\mathrm{Bn})\right]$}

After the previous reaction had shown that $\left[\mathrm{Cp}_{2} \mathrm{Zr}(\mathrm{NHPh})(\mathrm{Bn})\right]$ thermally ejects toluene to give the amide bridged dimer $\mathbf{1 8}$, the reaction was repeated in the presence of a germylene to investigate whether the germylene could act as a suitable donor or even react with the amide to the desired heterobimetallic. This approach would also avoid possible ligand exchange between [Ge(HMDS $\left.)_{2}\right]$ and the free amine, as was a possible side product as shown in the reaction in Scheme 35. Equal molar amounts of $\left[\mathrm{Cp}_{2} \mathrm{Zr}(\mathrm{NHPh})(\mathrm{Bn})\right]$ and $\left[\mathrm{Ge}(\mathrm{HMDS})_{2}\right]$ were dissolved in deuterated benzene heated to $40{ }^{\circ} \mathrm{C}$ in an NMR tube fitted with a Young's tap for 2 days, before being heated to $100{ }^{\circ} \mathrm{C}$.

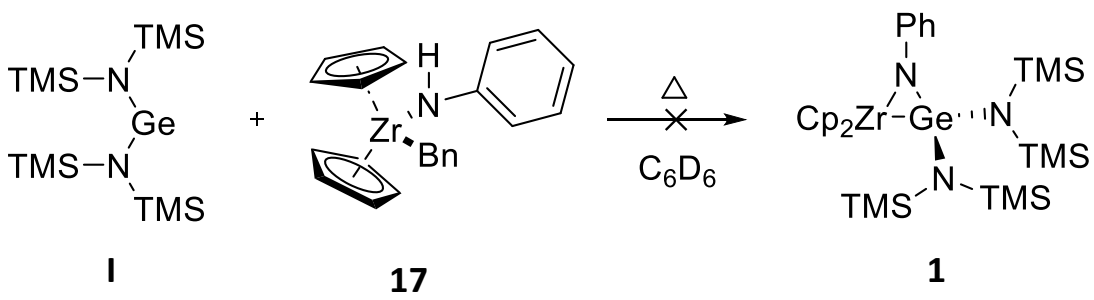

Analysis of the ${ }^{1} \mathrm{H}$ NMR spectra over the time period showed that the evolution of two small new cyclopentadienyl resonances at $\delta 5.67$ and $5.85 \mathrm{ppm}$. For the high field resonances, the intensity of the methylene peak at $\delta 2.22 \mathrm{ppm}$ decreased over time, while the methyl toluene resonance at $2.11 \mathrm{ppm}$ grew in over time, indicating the elimination of the benzyl group from $\left[\mathrm{Cp}_{2} \mathrm{Zr}(\mathrm{NHPh})(\mathrm{Bn})\right]$. The HMDS resonance of $\mathrm{I}$ at $\delta 0.32 \mathrm{ppm}$ decreased over time with the evolution of two new singlets in a 1:4 ratio at $\delta 0.53$ and $0.09 \mathrm{ppm}$ growing in over time. These peaks indicate that while most of the [Ge(HMDS $\left.)_{2}\right]$ is 
likely decomposing to the free ligand HMDS-H, a small fraction of I may have reacted. The ${ }^{1} \mathrm{H}$ NMR spectra does not allow this signal to be assigned with any certainty, but one could speculate that it could either be the complexation of the germylene to the zirconium centre in a donor-accepter relationship, or could be ligand exchange between the free HMDS-H and the phenyl amide to give the zirconium coordinated HMDS ligand and free aniline (Scheme 35).

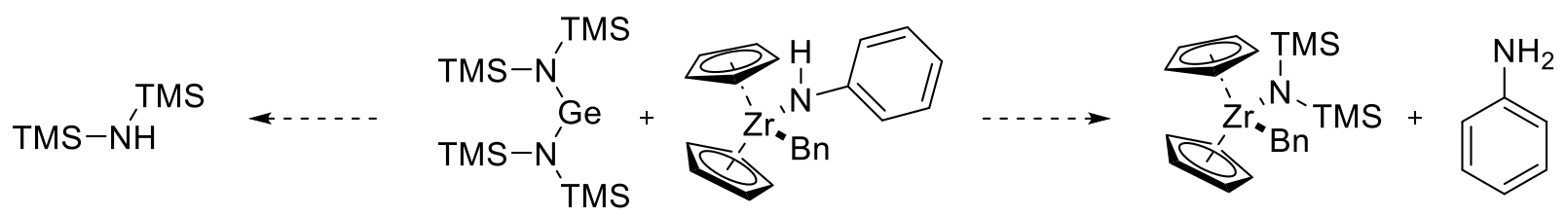

Scheme 35: Possible products of the reaction between phenylamide-benzylzirconocene and $\left[\mathrm{Ge}(\mathrm{HMDS})_{2}\right]$ at $100^{\circ} \mathrm{C}$.

The decomposition of $\left[\mathrm{Ge}(\mathrm{HMDS})_{2}\right.$ ] to give free protonated $\mathrm{HMDS}-\mathrm{H}$ requires an acid or proton transfer. The only acidic proton available in the reaction (other than minute amounts of impurities) is the acidic $\alpha$ proton of the phenyl amide. This proton is required for the elimination of the benzyl fragment as toluene so the migration to HMDS seems unlikely. Upon further heating a green crystalline solid identified as $\mathbf{1 8}$ by single crystal X-ray diffraction was isolated in a $70 \%$ yield.

\subsubsection{Reduction of $\left[\mathrm{Cp}_{2} \mathrm{ZrCl}\right]$ with magnesium in the presence of $\left[\mathrm{Ge}\left(\mathrm{BIAN}^{\mathrm{Mes}}\right)\right]$}

Applying the synthetic strategy employed by Muller et al. in their synthesis of donor stabilised germaniumzirconium bonds, the reduction of zirconocene dichloride in the presence of a germylene was attempted to try and form a direct zirconium germanium bond without the use of a bridging amide ligand.$^{60}$ After 24 hours there was no magnesium metal left in the reaction leaving a purple black solution (eq. 33). The reaction was dried to remove volatiles, extracted into toluene and filtered to give a dark purple solution.
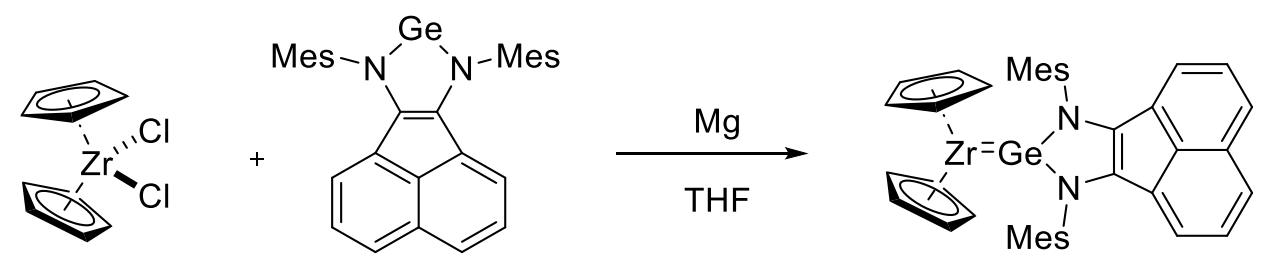
Analysis of the ${ }^{1} \mathrm{H}$ NMR spectrum of a dried aliquot of the purple solution showed the resonances of the free germylene in solution at $\delta 7.20$ (doublet), 6.94 (singlet), 6.90 (triplet), 6.59 (doublet) 2.29 (singlet) and $2.26 \mathrm{ppm}$ (singlet). Several resonances present in the chemical shift range $\delta 6.0-4.5 \mathrm{ppm}$ indicated a range of cyclopentadienyl environments and hence a range of products. The major resonance at $\delta 5.72$ ppm was shifted upfield from the starting material ( $\delta 5.89 \mathrm{ppm})$. The singlet integrated for 10 protons relative to the known germylene environments, indicating this unknown peak was the major product of the zirconocene dichloride reduction. Attempts to isolate and crystalise the compound attributed to this peak were not successful.

\subsubsection{Addition of $\left[(\mathrm{HMDS})_{2} \mathrm{Ge}(\mathrm{NMes})\right]$ to $\left[\mathrm{Cp}_{2} \mathrm{Zr}(\mathrm{Bu})_{2}\right]$}

In an attempt to synthesise 1 by the reaction of a germanimine with a reduced source of zirconium (Scheme 1), zirconocene dichloride was treated with 2 equivalents of $n$-BuLi at $-30{ }^{\circ} \mathrm{C}$ to generate the negishi reagent. After warming to room temperature over 30 minutes the orange solution was treated with a solution of in situ generated compound 9. After 2 days the solvent was removed the dark brown residue was extracted into hexane and filtered to give a dark yellow solution. ${ }^{1} \mathrm{H}$ NMR spectroscopy indicated two new major products. The two products proved inseparable.

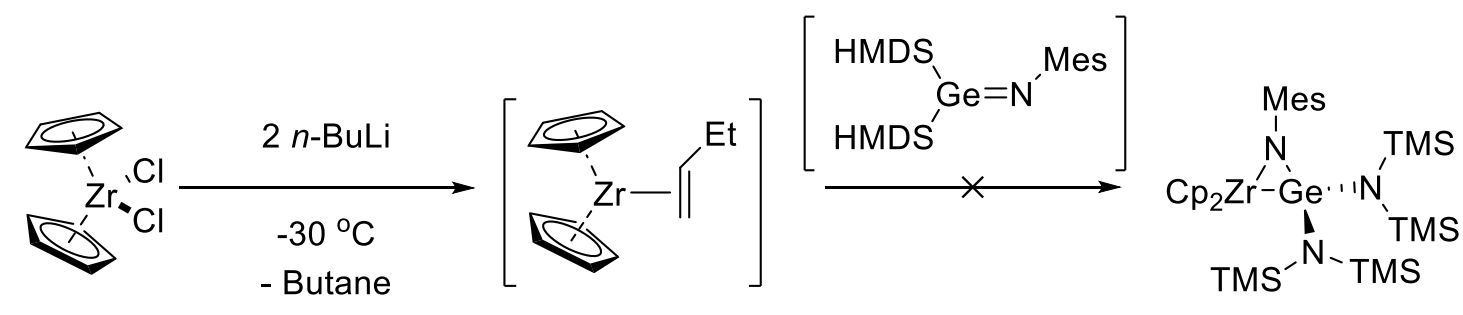

Scheme 36: Addition of germanimine 9 to the Negishi reagent.

\subsubsection{Reduction of $\left[\mathrm{Cp}_{2} \mathrm{ZrCl}_{2}\right]$ with magnesium in the presence of [(HMDS) $\left.{ }_{2} \mathrm{Ge}(\mathrm{NMes})\right]$}

In an attempt to synthesise $\mathbf{1}$ by the reaction of a germanimine with a reduced source of zirconium (Scheme 1), zirconocene dichloride was treated with magnesium metal in THF. This suspension was then then treated with an in situ generated solution of $\mathbf{9}$ in hexane. The suspension was left to stir for two days until no more solid magnesium was present, resulting in a dark orange solution. Following removal of the solvent the dark yellow residue was extracted into hexane and filtered to remove a black solid. ${ }^{1} \mathrm{H}$ NMR 
spectroscopy of the hexane extraction indicated a range of products. Isolation of these products was not possible.

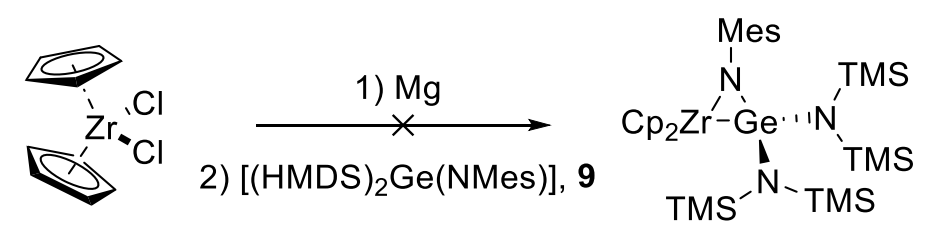

\subsection{Summary of Results}

The two synthetic routes to 1 were attempted; firstly, through the addition of a germylene to an imidozirconocene, secondly through the reactions of a germanimine with a $\operatorname{Zr}(I I)$ species. However, neither route lead to the synthesis of the desired heterobimetallic complex 1.

For the synthetic route to $\mathbf{1}$ from the imidozirconocene species it was proposed that the [2+2] cycloaddition amido-bridged tetracycle product could be explain the products observed by ${ }^{1} \mathrm{H}$ NMR spectroscopy. This is known to occur when there is insufficient steric bulk on the arylamide, to give the compounds XXIX and 18.

For the synthetic route to 1 from the germanimine, the reaction with the in situ generated Negishi reagent gave two observable products by ${ }^{1} \mathrm{H}$ NMR spectroscopy, though neither product indicated that compound 1 had been formed. Attempted isolation of these two compounds is ongoing.

In conclusion, the desired heterobimetallic complex 1 was not successfully synthesised from either of the theorised synthetic routes. However, as the chemistry of low valent group 14 compounds develops new and exciting synthetic methods, complex 1 is a viable synthetic target for the future. 


\section{Appendices}

\subsection{Experimental}

\subsubsection{General Procedures}

Unless otherwise stated all manipulations were performed under a dry and oxygen free $\mathrm{N}_{2}$ environment using Schlenk-line and cannula techniques, or in a nitrogen filled glovebox. Solvents were dried using a PureSolv. System (Innovative Technologies) and stored over $4 \AA$ molecular sieves. ${ }^{1} \mathrm{H}$ and ${ }^{13} \mathrm{C}$ spectra were recorded in deuterated benzene at $298 \mathrm{~K}$ using a Varian INOVA system at ${ }^{1} \mathrm{H}: 300$ and $600 \mathrm{MHz}$ or ${ }^{13} \mathrm{C}: 125$ $\mathrm{MHz}$ and internally referenced to residual solvent resonances. Elemental analyses were performed by $\mathrm{S}$. Boyer at London Metropolitan University. Infrared spectra were recorded on a Bruker Tensor 27 FTIR spectrometer using Nujol mineral oil. Absorption spectra were obtained on a Shimadzu UV-2600 Spectrophotometer. All chemicals were purchased from Sigma-Aldrich chemical company and used as received unless otherwise stated. Liquids were subjected to a minimum of three freeze-pump-thaw cycles before storage over $4 \AA$ A molecular sieves in the glovebox.

Samples suitable for X-ray diffraction were grown in a concentrated solution of the sample using at $-30{ }^{\circ} \mathrm{C}$ unless stated otherwise. Crystals were covered in inert oil and suitable crystals were selected under a microscope and mounted on an Agilent SuperNova X-ray Diffraction system fitted with an Atlas detector. Data were collected at the temperature indicated using focused Mo Ka radiation at $0.71073 \AA$ or $\mathrm{Cu}$ K $\alpha$ radiation at $1.54128 \AA$ as specified. Intensities were collected for Lorentz and polarisation effects and for absorption using multi-scan methods. Space groups were determined from systematic absences and checked for higher symmetry. All structures were solved using direct methods with SHELXT or SHELXL, refined on F2 using all data by full matrix least-squares procedures with SHELXL-97, within OLEX-2.3. ${ }^{125}$ Non-hydrogen atoms were refined with anisotropic displacement parameters. Hydrogen atoms were placed in calculated positions or manually assigned from residual electron density where appropriate unless otherwise stated. The functions minimized were $\Sigma w(F 20-81 F 2 c)$, with $w=[\sigma 2(F 20)+a P 2+b P]-1$, where $P=[\max (F o) 2+2 F 2 c] / 3$. The isotropic displacement parameters are 1.2 or 1.5 times the isotropic equivalent of their carrier atoms.

The lithium salts of the following: hexamethyldisilazane, 2,6 diisopropylphenylamine, 2,6 dimethylphenylamine, N1,N8-bis(trimethylsilyl)naphthalene-1,8-diamine, 2,6-dibenzhydryl-4-(tert- 
butyl)aniline and aniline were synthesised from the slow addition of $2.0 \mathrm{M} \mathrm{n}$-BuLi (4 mmol) or $2.0 \mathrm{M} \mathrm{n}$ BuLi $\left(8.0 \mathrm{mmol}\right.$ ) (for $N^{1}, N^{8}$-bis(trimethylsilyl)naphthalene) in cyclohexane to a solution of the amine (4 $\mathrm{mmol}$ ) in hexane at $-30^{\circ} \mathrm{C}$. The solvent was removed under reduced pressure to yield the products as white, yellow or red solids.

\subsection{2 $\left[\left(\mathrm{C}_{5} \mathrm{H}_{5}\right)_{2} \mathrm{ZrN}\left(2,6-{ }^{i} \mathrm{Pr}_{2} \mathrm{C}_{6} \mathrm{H}_{3}\right)\left(\mathrm{C}_{4} \mathrm{H}_{8} \mathrm{O}\right)\right] \quad\left[\mathrm{Cp}_{2} \mathrm{Zr}(\mathrm{NDipp})(\mathrm{THF})\right]$}

This compound was made with modification to the literature procedure. ${ }^{54}$ To an NMR tube fitted with a Young's tap was added [ $\left.\mathrm{Cp}_{2} \mathrm{Zr}(\mathrm{Me})(\mathrm{NHDipp})\right](22 \mathrm{mg}, 0.05 \mathrm{mmol})$ in $\mathrm{C}_{6} \mathrm{D}_{6}(300 \mu \mathrm{L})$ along with 2 drops of deuterated THF. The reaction was heated on an aluminium heating block for 5 days and monitored by ${ }^{1} \mathrm{H}$ NMR spectroscopy until no starting material remained. No yield was taken for this complex due to it being used immediately in subsequent reactions.

${ }^{1} \mathrm{H}$ NMR $\left(\mathrm{C}_{6} \mathrm{D}_{6}, 600 \mathrm{MHz}\right) \delta 7.22\left(\mathrm{~d}, \mathrm{~J}_{\mathrm{HH}}=7.2 \mathrm{~Hz}, 2 \mathrm{H}\right.$, meta-ArH); $6.90\left(\mathrm{t}, \mathrm{J}_{\mathrm{HH}}=7.4 \mathrm{~Hz}, 1 \mathrm{H}\right.$, para-ArH); $6.07(\mathrm{~s}$, $10 \mathrm{H}, \mathrm{C}_{5} \mathrm{H}_{5}$ ); 3.70 (sept, $\left.J_{\mathrm{HH}}=6.8 \mathrm{~Hz}, 2 \mathrm{H}, \mathrm{CH}\left(\mathrm{CH}_{3}\right)_{3}\right) ; 1.38\left(\mathrm{~d}, J_{\mathrm{HH}}=6.8 \mathrm{~Hz}, 12 \mathrm{H}\right.$ ). A small amount of starting material was still observable in the ${ }^{1} \mathrm{H}$ NMR spectrum as an impurity.

\subsection{3 $\left[2,4,6-(\mathrm{Me}) \mathrm{C}_{6} \mathrm{H}_{2} \mathrm{~N}_{3}\right] \quad\left[\mathrm{MesN}_{3}\right] \quad \mathrm{C}_{9} \mathrm{H}_{11} \mathrm{~N}_{3}$}

In a modification of the literature procedure, 2,4,6 trimethyl aniline $(6.80 \mathrm{~mL}, 0.05 \mathrm{~mol})$ was added to a $250 \mathrm{~mL} \mathrm{RB}$ (round-bottomed) flask with $9.7 \mathrm{~mL}$ of concentrated $\mathrm{HCl}\left(12 \mathrm{M}, 0.12 \mathrm{~mol}\right.$ ) and $50 \mathrm{~mL}$ of $\mathrm{H}_{2} \mathrm{O}$. The suspension was stirred for 30 minutes until a solution obtained. The solution was cooled to $0{ }^{\circ} \mathrm{C}$ with an ice bath and to the solution was added a prepared solution of sodium nitrite $(6.89 \mathrm{~g}, 0.10 \mathrm{~mol})$ in $\mathrm{H}_{2} \mathrm{O}$ $(5 \mathrm{~mL})$ dropwise over 2 minutes. The resulting solution was left to stir for 1 hour. The solution had the $\mathrm{pH}$ adjusted to 7 with saturated sodium bicarbonate solution. The solution was again cooled to $0{ }^{\circ} \mathrm{C}$ with an ice bath and treated with a solution of sodium azide $(6.50 \mathrm{~g}, 0.10 \mathrm{~mol})$ in $5 \mathrm{~mL}$ water dropwise over 10 minutes. The resulting solution was left to stir for 2 hours. The compound was extracted into diethyl ether $(2 \times 50 \mathrm{~mL})$, washed with $\mathrm{H}_{2} \mathrm{O}(2 \times 50 \mathrm{~mL})$ and saturated sodium chloride solution $(2 \times 50 \mathrm{~mL})$ before having the solvent removed under reduced pressure. Due to the potential risk of explosion a blast shield was used to isolate the azide any time the compound was put under reduced pressure. The resulting dark orange liquid was purified by vacuum distillation to give $\left[\mathrm{MesN}_{3}\right]$ as a dense yellow liquid. The solution discoloured over the next week so was stored under $\mathrm{N}_{2}$ in the glovebox at $-30{ }^{\circ} \mathrm{C}$ to minimise further degradation. ${ }^{1} \mathrm{H}$ NMR data for the compound agreed with literature values. ${ }^{126}$

Yield: $6.74 \mathrm{~g}(87 \%)$. 


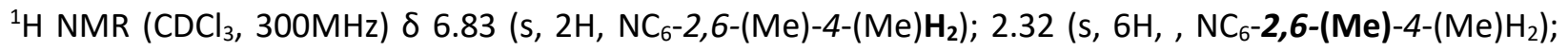
$2.25\left(\mathrm{~s}, 3 \mathrm{H}, \mathrm{NC}_{6}-2,6-(\mathrm{Me})-4-(\mathrm{Me}) \mathrm{H}_{2}\right)$.

\subsection{4 $\left[\mathrm{C}_{6} \mathrm{H}_{5} \mathrm{~N}_{3}\right] \quad\left[\mathrm{PhN}_{3}\right] \quad \mathrm{C}_{6} \mathrm{H}_{5} \mathrm{~N}_{3}$}

In a modification of the literature procedure, aniline ( $4.66 \mathrm{~g}, 0.05 \mathrm{~mol})$ was added to a $250 \mathrm{~mL}$ RB flask with $10.0 \mathrm{~mL}$ of concentrated $\mathrm{HCl}(12 \mathrm{M}, 0.12 \mathrm{~mol})$ in $\mathrm{H}_{2} \mathrm{O}(50 \mathrm{~mL})$. The solution was stirred for 30 minutes before being cooled to $0{ }^{\circ} \mathrm{C}$ with an ice bath. A solution of sodium nitrite $(4.82 \mathrm{~g}, 0.07 \mathrm{~mol})$ in $\mathrm{H}_{2} \mathrm{O}(4 \mathrm{~mL})$ was added dropwise over 2 minutes. The resulting solution was left to stir for 1 hour. The $\mathrm{pH}$ was adjusted to 7 sodium with sodium bicarbonate solution. The solution was cooled to $0{ }^{\circ} \mathrm{C}$ with an ice bath and treated with sodium azide $(5.20 \mathrm{~g}, 0.08 \mathrm{~mol})$ in $\mathrm{H}_{2} \mathrm{O}(2 \mathrm{~mL})$ dropwise over 10 minutes. The compound was extracted into diethyl ether $(2 \times 50 \mathrm{~mL})$, washed with $\mathrm{H}_{2} \mathrm{O}(2 \times 50 \mathrm{~mL})$ and saturated sodium chloride solution $(2 \times 50 \mathrm{~mL})$ before solvent removal under reduced pressure. Due to the potential risk of explosion a blast shield was used to isolate the azide any time it was put under reduced pressure. The resulting orange liquid was purified by vacuum distillation to give $\left[\mathrm{PhN}_{3}\right]$ as a yellow liquid. Due to the short lifetime of the azide the product was stored under $\mathrm{N}_{2}$ in the glovebox at $-30^{\circ} \mathrm{C}$ to minimise degradation. ${ }^{127}{ }^{1} \mathrm{H} \mathrm{NMR}$ data for the compound agreed with the literature values. ${ }^{128}$

Yield: $5.65 \mathrm{~g}(91 \%)$.

${ }^{1} \mathrm{H} \mathrm{NMR}\left(\mathrm{CDCl}_{3}, 300 \mathrm{MHz}\right) \delta 7.36\left(\mathrm{t}, \mathrm{J}_{\mathrm{HH}}=8.2 \mathrm{~Hz}, 2 \mathrm{H}\right.$, meta- $\left.\mathrm{C}_{6} \mathrm{H}_{5}\right) ; 7.15\left(\mathrm{t}, \mathrm{J}_{\mathrm{HH}}=8.1 \mathrm{~Hz}, 1 \mathrm{H}\right.$, para- $\left.\mathrm{C}_{6} \mathrm{H}_{5}\right) ; 7.04$ (d, $J_{\mathrm{HH}}=8.4 \mathrm{~Hz}, 2 \mathrm{H}$, ortho- $\mathrm{C}_{6} \mathrm{H}_{5}$ ).

\subsection{5 [2,6- $\left.\left(\left(\mathrm{C}_{6} \mathrm{H}_{5}\right)_{2} \mathrm{CH}\right)_{2}-4-\left(\left(\mathrm{CH}_{3}\right)_{3} \mathrm{C}\right) \mathrm{C}_{6} \mathrm{H}_{2} \mathrm{NH}_{2}\right] \quad\left[\mathrm{Ar}^{*} \mathrm{NH}_{2}\right] \quad \mathrm{C}_{36} \mathrm{H}_{35} \mathrm{~N}$}

This compound was made In a modification to the literature procedure. ${ }^{129}$ To a $250 \mathrm{~mL}$ RB flask with 4tert-buytl-aniline $(2.50 \mathrm{~g}, 0.02 \mathrm{~mol})$ was added 2 equivalents of diphenyl-methanol $(6.17 \mathrm{~g}, 0.03 \mathrm{~mol})$. The suspension was heated to $125^{\circ} \mathrm{C}$ for 40 minutes. The resulting solution was treated with $\left[\mathrm{ZnBr}_{2}\right](2.09 \mathrm{~g}$, $9.28 \mathrm{mmol})$ in $\mathrm{HCl}(12.1 \mathrm{M}, 1.4 \mathrm{~mL})$ and the reaction was left to stir overnight. The resulting crude solid was filtered and washed with methanol using a Buchner funnel and dried in a heating pistol for 1 week $\left(60^{\circ} \mathrm{C}\right)$ to give the compound as a white solid. The compound agreed with literature ${ }^{1} \mathrm{H}$ NMR spectral data.

${ }^{1} \mathrm{H} \mathrm{NMR}\left(\mathrm{CDCl}_{3}, 300 \mathrm{MHz}\right) \delta 7.29\left(\mathrm{t}, \mathrm{J}_{\mathrm{HH}}=7.5 \mathrm{~Hz}, 8 \mathrm{H}\right.$, meta- $\left.\mathrm{C}_{6} \mathrm{H}_{5}\right) ; 7.22\left(\mathrm{t}, J_{\mathrm{HH}}=7.3 \mathrm{~Hz}, 4 \mathrm{H}\right.$, para- $\left.\mathrm{C}_{6} \mathrm{H}_{5}\right) ; 7.10$ $\left(\mathrm{d}, J_{\mathrm{HH}}=7.3 \mathrm{~Hz}, 8 \mathrm{H}\right.$, ortho- $\left.\mathrm{C}_{6} \mathrm{H}_{5}\right) ; 6.58\left(\mathrm{~s}, 2 \mathrm{H}\right.$, meta- $\left.\mathrm{C}_{6} \mathrm{H}_{2}\right) ; 5.47\left(\mathrm{~s}, 2 \mathrm{H},\left(\mathrm{CHPh}_{2}\right)_{2}\right) ; 3.28\left(\mathrm{~s}, 2 \mathrm{H}, \mathrm{NH}_{2}\right) ; 0.98(\mathrm{~s}$, $\left.9 \mathrm{H}, \mathrm{C}\left(\mathrm{CH}_{3}\right)_{3}\right)$. 
${ }^{1} \mathrm{H}$ NMR $\left(\mathrm{C}_{6} \mathrm{D}_{6}, 300 \mathrm{MHz}\right)$ 8 7.15-6.99 (m, 20H, ArH); $6.93(\mathrm{~s}, 2 \mathrm{H}, \mathrm{ArH}) ; 5.47\left(\mathrm{~s}, 2 \mathrm{H},\left(\mathrm{CHPh}_{2}\right)_{2}\right) ; 1.11(\mathrm{~s}, 9 \mathrm{H}$, $\left.\mathrm{C}\left(\mathrm{CH}_{3}\right)_{3}\right)$.

\subsection{6 [2,6-(( $\left.\left.\left.\mathrm{C}_{6} \mathrm{H}_{5}\right)_{2} \mathrm{CH}\right)_{2}-4-\left(\left(\mathrm{CH}_{3}\right)_{3} \mathrm{C}\right) \mathrm{C}_{6} \mathrm{H}_{2} \mathrm{~N}_{3}\right] \quad\left[\mathrm{Ar} * \mathrm{~N}_{3}\right] \quad 25 \quad \mathrm{C}_{36} \mathrm{H}_{33} \mathrm{~N}_{3}$}

In a $250 \mathrm{~mL}$ RB flask with stirring, [Ar* $\mathrm{NH}_{2}$ ] $(2.68 \mathrm{~g}, 5.57 \mathrm{mmol})$ was dissolved in 1:2 $\mathrm{H}_{2} \mathrm{O}(50 \mathrm{~mL})$ to THF $(100 \mathrm{~mL})$ mixture. Concentrated $\mathrm{HCl}$ was added $(10 \mathrm{~mL}, 12 \mathrm{M})$ and the temperature of the reaction was brought down to $0{ }^{\circ} \mathrm{C}$ using an ice bath. The solution was treated with 4 equivalents of sodium nitrite (1.50 g, $22.3 \mathrm{mmol})$ in $\mathrm{H}_{2} \mathrm{O}(10 \mathrm{~mL})$ added dropwise over 5 minutes before being left to stir for four hours, after which time the solution had turned yellow. A solution of $\left[\mathrm{NaN}_{3}\right](1.44 \mathrm{~g}, 0.02 \mathrm{~mol})$ in $\mathrm{H}_{2} \mathrm{O}(10 \mathrm{~mL})$ was added dropwise over 5 minutes while maintaining an internal reaction temperature of under $5{ }^{\circ} \mathrm{C}$. The reaction was left to stir for four hours. The compound was extracted into toluene $(3 \times 50 \mathrm{~mL})$ and washed with sodium bicarbonate $(2 \times 100 \mathrm{~mL}), \mathrm{H}_{2} \mathrm{O}(2 \times 50 \mathrm{~mL})$ and brine $(2 \times 50 \mathrm{~mL})$ before the toluene fraction was dried over $\mathrm{MgSO}_{4}$ and filtered. The volatiles removed under reduced pressure to give the azide as an off white powder. A small amount ( $300 \mathrm{mg}$ ) of compound was recrystalised from the minumem value of toluene $\left(2 \mathrm{~mL}\right.$ ) for single crystal $\mathrm{X}$-ray diffraction. The compound was analysed by ${ }^{1} \mathrm{H}$ and ${ }^{13} \mathrm{C}$ NMR, liquid chromatography mass spectrometry (LC-MS) and single crystal X-ray diffraction.

Yield: $4.39 \mathrm{~g}(51 \%)$

${ }^{1} \mathrm{H} \mathrm{NMR}\left(\mathrm{CDCl}_{3}, 600 \mathrm{MHz}\right) \delta 7.28\left(\mathrm{t}, \mathrm{J}_{\mathrm{HH}}=7.3 \mathrm{~Hz}, 8 \mathrm{H}\right.$, meta $\left.-\mathrm{C}_{6} \mathrm{H}_{5}\right) ; 7.21\left(\mathrm{t}, \mathrm{J}_{\mathrm{HH}}=7.4 \mathrm{~Hz}, 4 \mathrm{H}\right.$, para $\left.-\mathrm{C}_{6} \mathrm{H}_{5}\right) ; 7.07$ $\left(\mathrm{d}, \mathrm{J}_{\mathrm{HH}}=7.0 \mathrm{~Hz}, 8 \mathrm{H}\right.$, ortho- $\left.\mathrm{C}_{6} \mathrm{H}_{5}\right) ; 6.80\left(\mathrm{~s}, 2 \mathrm{H}\right.$, meta- $\left.\mathrm{C}_{6} \mathrm{H}_{2}\right) ; 5.85\left(\mathrm{~s}, 2 \mathrm{H},\left(\mathrm{CHPh}_{2}\right)_{2}\right) ; 1.02\left(\mathrm{~s}, 9 \mathrm{H}, \mathrm{C}\left(\mathrm{CH}_{3}\right)_{3}\right)$.

$\left.{ }^{1} \mathrm{H} \mathrm{NMR}\left(\mathrm{C}_{6} \mathrm{D}_{6}\right), 600 \mathrm{MHz}\right)$ 8 7.14-7.08 (m, 18H, ArH); $7.01\left(\mathrm{t}, J_{\mathrm{HH}}=7.3 \mathrm{~Hz}, 4 \mathrm{H}, \mathrm{ArH}\right) ; 6.06\left(\mathrm{~s}, 2 \mathrm{H},\left(\mathrm{CHPh}_{2}\right)_{2}\right)$; $1.02\left(\mathrm{~s}, 9 \mathrm{H}, \mathrm{C}\left(\mathrm{CH}_{3}\right)_{3}\right)$.

${ }^{13} \mathrm{C}$ NMR (C $\left.\mathrm{C}_{6} \mathrm{D}_{6} 125 \mathrm{MHz}\right) \delta 149.1$ (ArC); $143.6(\mathrm{ArC}) ; 139.7$ (ArC); $135.4(\mathrm{ArC}) ; 129.8(\operatorname{ArC}) ; 128.8(\operatorname{ArC}) ;$ $126.9(\mathrm{ArC}) ; 126.7(\mathrm{ArC}) ; 53.3\left(\mathrm{CHPh}_{2}\right) ; 34.7\left(\mathrm{C}\left(\mathrm{CH}_{3}\right)_{3}\right) ; 31.1\left(\mathrm{C}\left(\mathrm{CH}_{3}\right)_{3}\right)$.

LC-MS (Q-TOF): $m / z$ [M+] calculated for $\mathrm{C}_{36} \mathrm{H}_{33} \mathrm{~N}_{3}$ : 507.2674; found: 507.2670 .

\subsection{7 $\left[\mathrm{Ge}(\mathrm{HMDS})_{2}\right] \quad\left[\left(\left(\mathrm{SiMe}_{3}\right)_{2} \mathrm{~N}\right)_{2} \mathrm{Ge}\right] \quad \mathrm{C}_{12} \mathrm{H}_{36} \mathrm{GeN}_{2} \mathrm{Si}_{4}$}

This compound was made with a modification to the literature procedure. ${ }^{130}$ To a solution of stirred [(HMDS)-H] $(600 \mathrm{mg}, 3.71 \mathrm{mmol})$ in hexane $(13 \mathrm{~mL})$ at $-30{ }^{\circ} \mathrm{C}$ was added one equivalent of $n$-Buli in cyclohexane solution $(1.49 \mathrm{~mL}, 3.71 \mathrm{mmol})$. The solution was allowed to come to room temperature and 
left to stir overnight. The solvent was removed under reduced pressure and the solid was redissolved in THF (6 mL). The new solution was added dropwise to a stirred solution of $\mathrm{GeCl}_{2}$.dioxane $(0.430 \mathrm{~g}, 1.85$ $\mathrm{mmol}$ ) at $-30^{\circ} \mathrm{C}$ in THF $(6 \mathrm{~mL})$. The solution was left to stir for 12 hours, over which time a strong orange colour developed. The solvent was removed under reduced pressure and the residue was extracted into hexane $(2 \times 5 \mathrm{~mL})$ and filtered through celite. The solvent was removed under reduced pressure to give an oily orange solid which was characterised by ${ }^{1} \mathrm{H}$ NMR spectroscopy. The compound was used without further purification.

${ }^{1} \mathrm{H} \mathrm{NMR}\left(\mathrm{C}_{6} \mathrm{D}_{6}, 300 \mathrm{MHz}\right) \delta 0.32\left(\mathrm{~s}, 36 \mathrm{H},\left[\mathrm{Ge}\left(\mathrm{N}(\mathrm{TMS})_{2}\right)_{2}\right]\right)$.

\subsection{8 $\left[\left(\mathrm{C}_{5} \mathrm{H}_{5}\right)_{2} \mathrm{Zr}(\mathrm{Me})_{2}\right]\left[\mathrm{Cp}_{2} \mathrm{ZrMe}_{2}\right] \mathrm{C}_{12} \mathrm{H}_{16} \mathrm{Zr}$}

This compound was synthesised in a modification to the literature procedure. ${ }^{131}$ To a solution of $\left[\mathrm{Cp}_{2} \mathrm{ZrCl}_{2}\right]$ (500 mg, $1.71 \mathrm{mmol})$ in THF $(15 \mathrm{~mL})$ at $-30^{\circ} \mathrm{C}$ was added 2.1 equivalents of MeLi (3M, $\left.1.19 \mathrm{~mL}, 3.59 \mathrm{mmol}\right)$ in dimethoxyethane. The solution was left to stir for a minimum of 6 hours before the volatiles were removed under reduced pressure. The residue was extracted into toluene $(3 \times 3 \mathrm{~mL})$ and filtered through celite to give a pale yellow/green solution. The solution had the solvent removed under reduced pressure to give the product as a white powder. ${ }^{1} \mathrm{H}$ NMR data agreed with literature values.

Yield: $349 \mathrm{mg}(81 \%)$

${ }^{1} \mathrm{H} \mathrm{NMR}\left(\mathrm{C}_{6} \mathrm{D}_{6}, 300 \mathrm{MHz}\right) \delta 5.71\left(\mathrm{~s}, 10 \mathrm{H}, \mathrm{C}_{5} \mathrm{H}_{5}\right) ;-0.12\left(\mathrm{~s}, 6 \mathrm{H}, \mathrm{ZrMe}_{2}\right)$.

\subsection{9 $\left[\left(\mathrm{C}_{5} \mathrm{H}_{5}\right)_{2} \mathrm{Zr}\left(\mathrm{CH}_{3}\right)(\mathrm{Cl})\right] \quad\left[\mathrm{Cp}_{2} \mathrm{Zr}(\mathrm{Me})(\mathrm{Cl})\right] \quad \mathrm{C}_{12} \mathrm{H}_{16} \mathrm{ZrCl}$}

This compound was made with a modification to the literature procedure. ${ }^{131}$ To a stirred solution of [Cp $\mathrm{CrMe}_{2}$ ] $(400 \mathrm{mg}, 1.59 \mathrm{mmol})$ in toluene $(5 \mathrm{~mL})$ was added 1 equivalent of trityl chloride $(440 \mathrm{mg}, 1.59$ $\mathrm{mmol}$ ) and the reaction was left to stir overnight. Over this time the solution went from pale yellow/green to bright yellow. The solvent was removed under reduced pressure and the residue was washed with hexane $(3 \times 10 \mathrm{~mL})$. The product was dried under reduced pressure to give the $\left[\mathrm{Cp}_{2} \mathrm{Zr}(\mathrm{Me})(\mathrm{Cl})\right]$ as an off white solid. ${ }^{1} \mathrm{H}$ NMR data agreed with the literature values.

Yield: $0.329 \mathrm{mg}$ (76\%).

${ }^{1} \mathrm{H} N M R\left(\mathrm{C}_{6} \mathrm{D}_{6}, 300 \mathrm{MHz}\right) \delta 5.74\left(\mathrm{~s}, 10 \mathrm{H}, \mathrm{C}_{5} \mathrm{H}_{5}\right) ; 0.45(\mathrm{~s}, 3 \mathrm{H}, \mathrm{ZrMe})$. 


\subsubsection{0 $\quad\left[\left(\mathrm{C}_{5} \mathrm{H}_{5}\right)_{2} \mathrm{Zr}\left(\mathrm{CH}_{2} \mathrm{C}_{6} \mathrm{H}_{5}\right)_{2}\right] \quad\left[\mathrm{Cp}_{2} \mathrm{ZrBn}_{2}\right] \quad \mathrm{C}_{24} \mathrm{H}_{24} \mathrm{Zr}$}

This compound was made using a modification to the literature procedure. ${ }^{132}$ To a stirred solution of $\left[\mathrm{Cp}_{2} \mathrm{ZrCl}_{2}\right](550 \mathrm{mg}, 1.71 \mathrm{mmol})$ in diethyl ether $(10 \mathrm{~mL})$ at $-30{ }^{\circ} \mathrm{C}$ was added a solution of benzylmagnesiumchloride in diethyl ether $(1 \mathrm{M}, 3.71 \mathrm{~mL}, 3.71 \mathrm{mmol})$. The resulting orange suspension was left to stir overnight before the solvent was removed under reduced pressure. The compound was extracted into hexane $(4 \times 5 \mathrm{~mL})$ and filtered through celite to give a vibrant orange solution. Removal of the solvent under reduced pressure gave $\left[\mathrm{Cp}_{2} \mathrm{ZrBn}_{2}\right]$ as an orange solid. ${ }^{1} \mathrm{H}$ NMR data agreed with literature values.

${ }^{1} \mathrm{H} \mathrm{NMR}\left(\mathrm{C}_{6} \mathrm{D}_{6}, 300 \mathrm{MHz}\right) \delta 7.25\left(\mathrm{t}, J_{\mathrm{HH}}=7.8 \mathrm{~Hz}, 4 \mathrm{H}\right.$, meta-ArH); $6.93\left(\mathrm{t}, J_{\mathrm{HH}}=7.5 \mathrm{~Hz}, 2 \mathrm{H}\right.$, para-ArH); 6.88 (d, $J_{\mathrm{HH}}=8.9 \mathrm{~Hz}, 4 \mathrm{H}$, ortho-ArH); $5.51\left(\mathrm{~s}, 10 \mathrm{H}, \mathrm{C}_{5} \mathrm{H}_{5}\right) ; 1.83\left(\mathrm{~s}, 4 \mathrm{H}, \mathrm{CH}_{2} \mathrm{Ph}\right)$.

\subsubsection{1 $\quad\left[\mathrm{Zr}\left(\mathrm{CH}_{2} \mathrm{C}_{6} \mathrm{H}_{5}\right)_{4}\right] \quad\left[\mathrm{Zr}(\mathrm{Bn})_{4}\right] \quad \mathrm{C}_{28} \mathrm{H}_{28} \mathrm{Zr}$}

This compound was made using a modification to the literature procedure. ${ }^{133}$ To a stirred suspension of $\left[\mathrm{ZrCl}_{4}\right](1.00 \mathrm{~g}, 4.29 \mathrm{mmol})$ in diethyl ether at $-78^{\circ} \mathrm{C}$ was added an excess of benzylmagnesiumchloride solution $(1 \mathrm{M}, 20 \mathrm{~mL}, 5 \mathrm{mmol})$. The solution was allowed to come to temperature overnight. The suspension was filtered through a glass frit packed with celite and the solvent was removed under reduced pressure. The residue was extracted into toluene $(4 \times 5 \mathrm{~mL})$, filtered through celite and left to crystalise at $-30^{\circ} \mathrm{C}$ to give the compound as intense orange crystals. Due to light sensitivity it is recommended this compound is stored in a light free environment. The compound was confirmed as $\left[\mathrm{Zr}(\mathrm{Bn})_{4}\right]$ by ${ }^{1} \mathrm{H}$ NMR spectroscopy.

${ }^{1} \mathrm{H}$ NMR $\left(\mathrm{C}_{6} \mathrm{D}_{6}, 300 \mathrm{MHz}\right) \delta 7.06(\mathrm{~m}, 12 \mathrm{H}, \mathrm{ArH}) ; 6.39$ (d, JHH $=7.3 \mathrm{~Hz}, 8 \mathrm{H}$, ortho-ArH); 1.54 (s, 8H, $\left.\mathrm{CH}_{2} \mathrm{Ph}\right)$.

\subsubsection{Bis(trimethylsilyl)-4,10-bis((trimethylsilyl)imino)-4,10- dihydroperylene-3,9-diamine}

A previously prepared sample of [ $\left.\mathrm{Li}_{2} \mathrm{NAPH}\right](260 \mathrm{mg}, 0.85 \mathrm{mmol})$ in THF $(7 \mathrm{~mL})$ was added dropwise to a stirred solution of $\left[\mathrm{PbCl}_{2}\right](230 \mathrm{mg}, 0.85 \mathrm{mmol})$ in THF $(5 \mathrm{~mL})$ at $-30{ }^{\circ} \mathrm{C}$ and the solution was left to stir overnight. The solvent was removed under reduced pressure and the compound was extracted into toluene $(3 \times 5 \mathrm{~mL})$. The extraction was filtered through celite to give a yellow solution which was concentrated and stored at $-30{ }^{\circ} \mathrm{C}$ to crystalise. Over several days the solution went dark green. The 
solvent was removed under reduced pressure to give the named compound. ${ }^{1} \mathrm{H}$ NMR spectroscopy resonances agree with the literature values. ${ }^{69}$

${ }^{1} \mathrm{H}$ NMR $\left(\mathrm{C}_{6} \mathrm{D}_{6}, 600 \mathrm{MHz}\right) \delta 14.13(\mathrm{~s}, 2 \mathrm{H}, \mathrm{NH}) ; 8.13\left(\mathrm{~d}, 4 \mathrm{H}, J_{\mathrm{HH}}=10.0 \mathrm{~Hz}, \mathrm{C}_{20} \mathrm{H}_{8}\right) ; 7.19\left(\mathrm{~d}, J_{\mathrm{HH}}=9.7 \mathrm{~Hz}, 4 \mathrm{H}\right.$, $\left.\mathrm{C}_{20} \mathrm{H}_{8}\right) ; 0.48\left(\mathrm{~s}, 36 \mathrm{H},\left(\mathrm{SiMe}_{3}\right)_{4}\right)$.

\subsubsection{3 $\quad\left[\mathrm{Zr}\left(2,6-\left(\left(\mathrm{CH}_{3}\right)_{2} \mathrm{CH}\right)_{2} \mathrm{NHC}_{6} \mathrm{H}_{4}\right)_{4}\right] \quad\left[\mathrm{Zr}(\mathrm{NHDipp})_{4}\right] \quad \mathrm{C}_{48} \mathrm{H}_{72} \mathrm{Zr}$}

This compound was made using a modification to the literature procedure. ${ }^{134}$ To a solution of $\left[\mathrm{Zr}(\mathrm{Bn})_{4}\right]$ (50 mg, $0.11 \mathrm{mmol})$ in $\mathrm{C}_{6} \mathrm{D}_{6}(0.20 \mathrm{~mL})$ was added 4 equivalents of 2,6-diisopropylaniline $(77 \mathrm{mg}, 0.44 \mathrm{mmol}$, $0.08 \mathrm{~mL}$ ). The solution went from orange to a vibrant yellow. The solution was transferred into an NMR tube fitted with a Young's tap and heated to $100^{\circ} \mathrm{C}$ for 3 days until only one product was identifiable by ${ }^{1}$ H NMR spectrscopy. No yield was taken as the product was used immediately for another reaction.

${ }^{1} \mathrm{H}$ NMR $\left(\mathrm{C}_{6} \mathrm{D}_{6}, 300 \mathrm{MHz}\right) \delta$ 7.14-6.85 (m, 12H, ArH); $6.98(\mathrm{~s}, 4 \mathrm{H}, \mathrm{ZrNH}) ; 3.26\left(\mathrm{sep}, J_{\mathrm{HH}}=7.3 \mathrm{~Hz}, 8 \mathrm{H}\right.$, $\left.\left(\mathrm{CH}\left(\mathrm{CH}_{3}\right)_{2}\right)_{2}\right) ; 1.18\left(\mathrm{~d}, \mathrm{~J}_{\mathrm{HH}}=6.8 \mathrm{~Hz}, 48 \mathrm{H},\left(\mathrm{CH}\left(\mathrm{CH}_{3}\right)_{2}\right)_{2}\right)$.

\subsubsection{4 [ $\quad\left[\mathrm{Ge}\left(\left(2,6-\left(\left(\mathrm{CH}_{3}\right)_{2} \mathrm{CH}\right)_{2} \mathrm{NC}_{6} \mathrm{H}_{4}\right)_{3}\right] \quad\left[(\mathrm{GeNDipp})_{3}\right]\right.$ $\mathrm{C}_{36} \mathrm{H}_{51} \mathrm{Ge}_{3} \mathrm{~N}_{3}$}

This compound was made using a modification from the literature procedure. ${ }^{135}$ To a solution of [Zr(NHDipp $)_{4}$ ] $(88 \mathrm{mg}, 0.11 \mathrm{mmol})$ in $\mathrm{C}_{6} \mathrm{D}_{6}(0.30 \mathrm{~mL})$ was added 2 equivalents of [Ge(NAPH $\left.{ }^{\mathrm{TMS}}\right)$ ] $(86 \mathrm{mg}$, $0.22 \mathrm{mmol})$. The solution was transferred into an NMR tube fitted with a Young's tap and heated $50{ }^{\circ} \mathrm{C}$ for 5 days. The NMR tube was taken into a glove box and the solvent was removed under reduced pressure. The residue was extracted into minimal toluene $(3 \mathrm{~mL})$ and heated to give a clear solution. This solution was transferred to the $-30^{\circ} \mathrm{C}$ freezer to crystalise. The named compound was identified by single crystal X-ray crystallography and ${ }^{1} \mathrm{H}$ NMR spectroscopy. ${ }^{1} \mathrm{H}$ NMR spectroscopy resonances agreed with literature values. $^{135}$

${ }^{1} \mathrm{H}$ NMR $\left(\mathrm{C}_{6} \mathrm{D}_{6}, 300 \mathrm{MHz}\right) \delta 7.16\left(\mathrm{~m}, 6 \mathrm{H}\right.$, meta-ArH); $7.10\left(\mathrm{~m}, 3 \mathrm{H}\right.$, para-ArH); $3.40\left(\mathrm{~s}, 6 \mathrm{H},\left(\mathrm{CHMe}_{2}\right)_{2}\right) ; 1.12$ $\left(\mathrm{d}, J_{\mathrm{HH}}=6.6 \mathrm{~Hz}, 36 \mathrm{H},\left(\mathrm{CHMe}_{2}\right)_{2}\right)$.

\subsubsection{5 $\quad\left[((\mathrm{TMS}) \mathrm{NH})_{2} \mathrm{C}_{10} \mathrm{H}_{6}\right] \quad\left[\mathrm{NAPH}^{\mathrm{TMS}}-\mathrm{H}\right]$}

This compound was made in a modification to the literature procedure. ${ }^{136}$ To a stirred solution of 1,8 diaminonaphthalene $(3.98 \mathrm{~g}, 25 \mathrm{mmol})$ in THF $(25 \mathrm{~mL})$ at $-30{ }^{\circ} \mathrm{C}$ was added MeLi $(16.1 \mathrm{~mL}, 50 \mathrm{mmol})$ 
dropwise by cannula transfer resulting in a green solution after 1 hour. The solution was heated to reflux then cooled to $-30{ }^{\circ} \mathrm{C}$. TMSCl $(6.35 \mathrm{~mL}, 67 \mathrm{mmol})$ was added dropwise by cannula transfer. The solution turned purple and was left to stir overnight. The solution was concentrated to half volume and cooled to $-30{ }^{\circ} \mathrm{C}$ to give $\left[((\mathrm{TMS}) \mathrm{NH})_{2} \mathrm{C}_{10} \mathrm{H}_{6}\right]\left(\mathrm{NAPH}^{\mathrm{TMS}}-\mathrm{H}\right)$ as colourless crystals.

Yield 3.86g (51\%)

${ }^{1} \mathrm{H} \mathrm{NMR}\left(\mathrm{CDCl}_{3}, 300 \mathrm{MHz}\right) \delta 7.15(\mathrm{~m}, 4 \mathrm{H}, \mathrm{ArH}) ; 6.71$ (dd, $\left.\mathrm{J}_{\mathrm{HH}}=6.8,1.8 \mathrm{~Hz}, 2 \mathrm{H}, \mathrm{ArH}\right) ; 5.55$ (br s, 2H, NH); 0.29 (s, 18H, TMS).

${ }^{1} \mathrm{H}$ NMR $\left(\mathrm{C}_{6} \mathrm{D}_{6}, 300 \mathrm{MHz}\right) \delta 7.36\left(\mathrm{~d}, J_{\mathrm{HH}}=7.9 \mathrm{~Hz}, 2 \mathrm{H}, \operatorname{ArH}\right) ; 7.20\left(\mathrm{t}, J_{\mathrm{HH}}=7.2 \mathrm{~Hz}, 2 \mathrm{H}, \operatorname{ArH}\right) ; 6.72\left(\mathrm{~d}, J_{\mathrm{HH}}=7.6\right.$ $\mathrm{Hz}, 2 \mathrm{H}, \mathrm{ArH}) ; 5.39$ (s, 2H, NH); 0.18 (s, 18H, TMS).

\subsubsection{6 $\left[\mathrm{Ge}\left(\left(\mathrm{N}\left(\mathrm{SiMe}_{3}\right)\right)_{2} \mathrm{C}_{10} \mathrm{H}_{6}\right)\right]\left[\mathrm{Ge}\left(\mathrm{NAPH}^{\mathrm{TMS}}\right)\right] \quad 3 \mathrm{C}_{16} \mathrm{H}_{24} \mathrm{GeN}_{2} \mathrm{Si}_{2}$}

A stirred solution of [NAPH $\left.{ }^{\mathrm{TMS}}-\mathrm{H}\right](500 \mathrm{mg}, 1.65 \mathrm{mmol})$ in $\mathrm{Et}_{2} \mathrm{O}(5 \mathrm{~mL})$ at $-30{ }^{\circ} \mathrm{C}$ was treated with $n$-BuLi (1.32 mL, 2.5 M in cyclohexane) dropwise over 3 minutes. The resulting solution was left to stir for 1 hour at room temperature before the solution was added dropwise to a solution of [ $\mathrm{GeCl}_{2}$.dioxane] (380 $\mathrm{mg}$, $1.65 \mathrm{mmol}$ ) in THF ( $8 \mathrm{~mL}$ ). The pale yellow solution was left to stir for 15 hours before having the solvent removed under reduced pressure. The compound was extracted into toluene $(10 \mathrm{~mL})$, filtered through celite and concentrated to $2 \mathrm{~mL}$. After 24 hours at $-30{ }^{\circ} \mathrm{C}$ orange crystals were deposited, identified as 3 by single crystal X-ray diffraction. Yield $0.45 \mathrm{~g}(73 \%)$.

${ }^{1} \mathrm{H} \mathrm{NMR}\left(\mathrm{C}_{6} \mathrm{D}_{6}, 600 \mathrm{MHz}\right) \delta 7.21\left(\mathrm{dd}, J_{\mathrm{HH}}=8.3,0.8 \mathrm{~Hz}, 2 \mathrm{H}, p-\mathrm{CH}\right), 7.13\left(\mathrm{t}, J_{\mathrm{HH}}=8.6 \mathrm{~Hz}, 2 \mathrm{H}, m-\mathrm{CH}\right), 6.71(\mathrm{dd}$, $\left.\left.J_{\mathrm{HH}}=7.1,0.9 \mathrm{~Hz}, 2 \mathrm{H}, \mathrm{o}-\mathrm{CH}\right), 0.34\left(\mathrm{~s}, 18 \mathrm{H}, \mathrm{Si}\left(\mathrm{CH}_{3}\right)_{3}\right)\right)$.

${ }^{13} \mathrm{C}$ NMR $\left.\left(\mathrm{C}_{6} \mathrm{D}_{6}, 125 \mathrm{MHz}\right) \delta 142.3,137.6,128.0,125.5,120.5,112.3\left(\mathrm{C}_{10} \mathrm{H}_{6}\right), 2.5\left(\mathrm{SiCH}_{3}\right)_{3}\right)$.

UV-vis (Hexane, $\left.25^{\circ} \mathrm{C}\right)\left[\lambda_{\max }, \mathrm{nm}\left(\varepsilon, \mathrm{mol}^{-1} \mathrm{~L} \mathrm{~cm}^{-1}\right)\right]: 235(33,800), 349(13,500)$.

Elemental analysis was not able to be obtained due to the extreme oxophilicity of this compound.

\subsubsection{7 $\left[\mathrm{Ge}\left(\mathrm{N}\left(2,4,6-\mathrm{CH}_{3}\left(\mathrm{C}_{6} \mathrm{H}_{2}\right)_{2}\left(\mathrm{C}_{12} \mathrm{H}_{6}\right)\right] \quad\left[\mathrm{Ge}\left(\mathrm{BIAN}{ }^{M e s}\right)\right] \quad 2 \quad \mathrm{C}_{30} \mathrm{H}_{28} \mathrm{GeN}_{2}\right.\right.$}

A stirred solution of $\left[\mathrm{GeCl}_{2}\right.$.dioxane] $(167 \mathrm{mg}, 0.67 \mathrm{mmol})$ in THF $(5 \mathrm{~mL})$ was treated with $\left[\mathrm{Mg}\left(\mathrm{BIAN}^{\mathrm{Mes}}\right)(\mathrm{THF})_{3}\right](490 \mathrm{mg}, 0.67 \mathrm{mmol})$ in $\mathrm{THF}(5 \mathrm{~mL})$ at $-30^{\circ} \mathrm{C}$. The solution changed from dark green to a dark brown over 4 hours. The reaction was left to stir overnight before being concentrated to dryness under reduced pressure. The residue was extracted into toluene and filtered through celite to give a dark 
brown solution. This solution was concentrated and cooled to $-30{ }^{\circ} \mathrm{C}$ to give dark brown crystals. Yield: $0.247 \mathrm{~g}(74 \%)$.

${ }^{1} \mathrm{H}$ NMR $\left(\mathrm{C}_{6} \mathrm{D}_{6}, 600 \mathrm{MHz}\right) \delta 7.20\left(\mathrm{~d}, J_{\mathrm{HH}}=7.6 \mathrm{~Hz}, 2 \mathrm{H}, p-\mathrm{CH}\right) ; 6.94\left(\mathrm{~s}, 4 \mathrm{H}\left(\mathrm{C}_{6} \mathrm{H}_{2}\right)_{2}\right) ; 6.90\left(\mathrm{t}, J_{\mathrm{HH}}=7.4 \mathrm{~Hz}, 2 \mathrm{H}, m-\right.$ $\mathrm{CH}) ; 6.59\left(\mathrm{~d}, \mathrm{~J}_{\mathrm{HH}}=6.6 \mathrm{~Hz}, 2 \mathrm{H}, o-\mathrm{CH}\right) ; 2.29\left(\mathrm{~s}, 12 \mathrm{H},\left(o-\mathrm{CH}_{3}\right)_{2}\left(\mathrm{C}_{6} \mathrm{H}_{2}\left(p-\mathrm{CH}_{3}\right)\right) ; 2.26\left(\mathrm{~s}, 6 \mathrm{H},\left(o-\mathrm{CH}_{3}\right)_{2}\left(\mathrm{C}_{6} \mathrm{H}_{2}\left(p-\mathrm{CH}_{3}\right)\right)\right.\right.$.

${ }^{13} \mathrm{C}$ NMR $\left(\mathrm{C}_{6} \mathrm{D}_{6}, 125 \mathrm{MHz}\right) \delta 140.1(\mathrm{~N}-\mathrm{C}), 138.9,136.01,134.2,132.0,132.1,129.6,129.4,127.7,125.6$, $118.2,21.2,18.7$.

Anal. Calcd. for $\mathrm{C}_{30} \mathrm{H}_{28} \mathrm{GeN}_{2}$ : C, 73.66; $\mathrm{H}, 5.77 ; \mathrm{N}, 5.73$. Found: $\mathrm{C}, 70.02 ; \mathrm{H}, 5.43 ; \mathrm{N}, 5.09$.

UV-vis (Hexane, $\left.25^{\circ} \mathrm{C}\right)\left[\lambda_{\max }, \mathrm{nm}\left(\varepsilon, \mathrm{mol}^{-1} \mathrm{~L} \mathrm{~cm}^{-1}\right)\right]: 324(15,900), 422(16,800)$.

\subsubsection{8 [Ge(NAPH $\left.\left.{ }^{\mathrm{TMS}}\right) \mathrm{Ge}\left(\mathrm{NAPH}^{\mathrm{TMS}}\right)(\mathrm{Bu})(\mathrm{NAPH})\right]\left[\mathrm{Li}(\mathrm{THF})_{4}\right] \quad 4$ $\mathrm{C}_{52} \mathrm{H}_{86} \mathrm{Ge}_{2} \mathrm{~N}_{4} \mathrm{Si}_{4} \mathrm{LiO}_{4}$}

\subsubsection{Method A:}

$n$-BuLi $(2.0 \mathrm{~mL}, 4.0 \mathrm{mmol})$ was added dropwise to a purple stirred solution of [NAPH ${ }^{\mathrm{TMS}}-\mathrm{H}$ ] $(610 \mathrm{mg}, 2.0$ $\mathrm{mmol})$ at $-30^{\circ} \mathrm{C}$ in $\mathrm{Et}_{2} \mathrm{O}(7 \mathrm{~mL})$ and left for 1 hour. The resulting solution was added dropwise to a stirred solution of $\left[\mathrm{GeCl}_{2}\right.$.dioxane] $(460 \mathrm{mg}, 2.0 \mathrm{mmol})$ in THF $(5 \mathrm{~mL})$ at $-30{ }^{\circ} \mathrm{C}$. The solution was left to stir overnight before the solvent was removed under reduced pressure. The residue was extracted with toluene $(2 \times 5 \mathrm{~mL})$, filtered and reduced to dryness.

Yield: $620 \mathrm{mg}(56 \%)$

\subsubsection{Method B}

To a solution of [Ge(NAPH $\left.\left.{ }^{\mathrm{TMS}}\right)\right](40 \mathrm{mg}, 0.11 \mathrm{mmol})$ in THF $(5 \mathrm{~mL})$ was added 0.5 equivalents of $n$-BuLi solution in cyclohexane $(0.03 \mathrm{~mL}, 0.05 \mathrm{mmol})$. The solution went from a dark orange to yellow. The solution was concentrated and stored in the freezer resulting in yellow crystals. Yield $38 \mathrm{mg}(63 \%)$.

${ }^{1} \mathrm{H}$ NMR $\left(\mathrm{C}_{6} \mathrm{D}_{6}, 600 \mathrm{MHz}\right) \delta 7.27\left(\mathrm{~d}, \mathrm{~J}_{\mathrm{HH}}=7.4 \mathrm{~Hz}, 2 \mathrm{H}\right) ; 7.22\left(\mathrm{~d}, \mathrm{~J}_{\mathrm{HH}}=8.0 \mathrm{~Hz}, 2 \mathrm{H}\right) ; 7.15-7.12(\mathrm{~m}, 4 \mathrm{H}) ; 6.93(\mathrm{~d}$, $\left.J_{H H}=8.1 \mathrm{~Hz}, 2 \mathrm{H}\right) ; 6.84\left(\mathrm{~d}, J_{\mathrm{HH}}=6.98 \mathrm{~Hz}, 2 \mathrm{H}\right), 3.29(\mathrm{~m}, 8 \mathrm{H}) ; 1.23(\mathrm{~m}, 8 \mathrm{H}) ; 1.19-1.14(\mathrm{~m}, 2 \mathrm{H}) ; 0.75\left(\mathrm{t}, J_{\mathrm{HH}}=\right.$ $6.54 \mathrm{~Hz}, 3 \mathrm{H}) ; 0.54(\mathrm{~s}, 18 \mathrm{H}) ; 0.50(\mathrm{~m}, 4 \mathrm{H}) ; 0.44(\mathrm{~s}, 18 \mathrm{H})$.

${ }^{13} \mathrm{C}$ NMR $\left(\mathrm{C}_{6} \mathrm{D}_{6}, 125 \mathrm{MHz}\right) \delta 149.3(\mathrm{ArC}) ; 138.3(\operatorname{ArC}) ; 137.7(\operatorname{ArC}) ; 128.4(\operatorname{ArC}) ; 126.0(\operatorname{ArC}) ; 125.7(\operatorname{ArC})$; 125.3 (ArC); 120.5 (ArC); 120.2 (ArC); $116.7(\operatorname{ArC}) ; 113.8(\operatorname{ArC}) ; 27.8 ; 27.0 ; 25.5 ; 15.6 ; 14.2 ; 3.3$. Other aromatic carbon signal assumed to be obscured by solvent resonance. 


\subsubsection{9 [Ge(NAPH $\left.\left.{ }^{\mathrm{TMS}}\right) \mathrm{Ge}(\mathrm{NAPH})(\mathrm{Me})\left(\mathrm{NAPH}^{\mathrm{TMS}}\right)\right]\left[\mathrm{Li}(\mathrm{THF})_{4}\right] \quad 5$ $\mathrm{C}_{52} \mathrm{H}_{86} \mathrm{Ge}_{2} \mathrm{~N}_{4} \mathrm{Si}_{4} \mathrm{LiO}_{4}$}

\subsubsection{Method A:}

MeLi (1.00 mL, $3.1 \mathrm{mmol}, 3.1 \mathrm{M}$ in dimethoxyethane) was added dropwise to a stirred solution of [NAPH ${ }^{\mathrm{TMS}}-\mathrm{H}$ ] (400 mg, $\left.1.32 \mathrm{mmol}\right)$ at $-30^{\circ} \mathrm{C}_{\text {in }} \mathrm{Et}_{2} \mathrm{O}(7 \mathrm{~mL})$ and left for 1 hour. The resulting solution was added dropwise to a stirred solution of $\mathrm{GeCl}_{2}$.dioxane $\left(306 \mathrm{mg}, 1.32 \mathrm{mmol}\right.$ ) in THF $(5 \mathrm{~mL})$ at $-30{ }^{\circ} \mathrm{C}$. The reaction was left to stir overnight before the solvents were removed in vacuo. The residue was extracted with toluene $(2 \times 5 \mathrm{~mL})$, filtered through celite and concentrated to dryness to give the compound as a yellow powder.

Yield: $132 \mathrm{mg}(19 \%)$

\subsubsection{Method B}

To a solution of 2 (46 mg, $0.04 \mathrm{mmol})$ in THF $(5 \mathrm{~mL})$ was added 0.5 equivalents of $n$-BuLi solution (0.02 $\mathrm{mmol}, 0.01 \mathrm{~mL}$ ). The solution went from a dark orange to yellow over several hours. The solution was concentrated to dryness to give a yellow solid. Yield: $26 \mathrm{mg}(62 \%)$.

${ }^{1} \mathrm{H}$ NMR $\left(\mathrm{C}_{6} \mathrm{D}_{6}, 600 \mathrm{MHz}\right) \delta 7.34\left(\mathrm{~d},, J_{\mathrm{HH}}=8.4 \mathrm{~Hz}, 2 \mathrm{H}\right) ; 7.25\left(\mathrm{~d}, J_{\mathrm{HH}}=8.9 \mathrm{~Hz}, 2 \mathrm{H}\right) ; 7.21\left(\mathrm{t}, J_{\mathrm{HH}}=7.7 \mathrm{~Hz}, 2 \mathrm{H}\right)$; $7.15-7.13(\mathrm{~m}, 2 \mathrm{H}) ; 7.03-6.99(\mathrm{~m}, 2 \mathrm{H}) ; 6.85\left(\mathrm{~d}, \mathrm{~J}_{\mathrm{HH}}=7.7 \mathrm{~Hz}, 2 \mathrm{H}\right), 3.11(\mathrm{~m}, 8 \mathrm{H}) ; 1.09(\mathrm{~m}, 8 \mathrm{H}) ; 0.38(\mathrm{~s}$, $18 \mathrm{H}) ; 0.37$ (s, 18H); -0.03 (br s, 3H).

${ }^{13} \mathrm{C}$ NMR $\left(\mathrm{C}_{6} \mathrm{D}_{6}, 125 \mathrm{MHz}\right) \delta 150.7(\mathrm{ArC}) ; 138.3(\operatorname{ArC}) ; 137.5(\operatorname{ArC}) ; 134.3(\operatorname{ArC}) ; 134.3(\operatorname{ArC}) ; 128.0(\operatorname{ArC}) ;$ $125.3(\operatorname{ArC}) ; 124.8(\operatorname{ArC}) ; 120.4(\operatorname{ArC}) ; 120.2(\operatorname{ArC}) ; 113.8(\operatorname{ArC}) ; 67.8 ; 65.5 ; 63.8 ; 24.8 ; 15.1 ; 3.1 ; 3.0$. Other aromatic carbon signal assumed to be present under the solvent signal.

\subsubsection{0 $\left[\left(\mathrm{Ge}\left(2,6-\mathrm{CH}\left(\mathrm{C}_{6} \mathrm{H}_{5}\right)_{2}\right)-4-\left(\mathrm{C}\left(\mathrm{CH}_{3}\right)_{3}\right) \mathrm{C}_{6} \mathrm{H}_{2} \mathrm{~N}\right)_{2}\right] \quad\left[\left(\mathrm{Ge}\left(\mathrm{NAr}^{*}\right)\right)_{2}\right] \quad 6$ $\mathrm{C}_{72} \mathrm{H}_{66} \mathrm{Ge}_{2} \mathrm{~N}_{2}$}

To a solution of [ $\mathrm{GeCl}_{2}$.dioxane] (48 mg, $0.21 \mathrm{mmol}$ ) in THF $(5 \mathrm{~mL}$ ) was added two equivalents of [LiNHAr*] $(0.20 \mathrm{~g}, 0.42 \mathrm{mmol})$ in THF $(5 \mathrm{~mL})$. The solution turned yellow overnight. The solvent was removed under reduced pressure and the residue was extracted with toluene $(3 \times 5 \mathrm{~mL})$. The solution was filtered through celite, then concentrated under reduced pressure before being transferred to a $-30^{\circ} \mathrm{C}$ freezer from which $\mathrm{X}$-ray quality crystals were grown. 
Yield $0.72 \mathrm{~g}, 31 \%$.

${ }^{1} \mathrm{H}$ NMR $\left(\mathrm{C}_{6} \mathrm{D}_{6}, 600 \mathrm{MHz}\right)$ ठ 7.27-7.25 (m, 12H); 7.15-7.11 (m, 16H); 7.06-7.00 (m, 16H), $6.43(\mathrm{~s}, 4 \mathrm{H}) ; 1.13$ $(\mathrm{s}, 18 \mathrm{H})$.

${ }^{13} \mathrm{C} \mathrm{NMR}\left(\mathrm{C}_{6} \mathrm{D}_{6}, 125 \mathrm{MHz}\right) \delta 145.7,138.5,130.4,129.9,129.2,127.0,125.7,125.5,54.2\left(\mathrm{CHPh}_{2}\right), 31.6\left(\mathrm{CH}_{3}\right)$, $30.1\left(\mathrm{CCH}_{3}\right)$.

\subsubsection{1 $\left[(\mathrm{Cl}) \mathrm{Ge}\left(\mathrm{N}(\mathrm{Cy}) \mathrm{C}(\mathrm{NHCy}) \mathrm{NAr}^{*}\right)\right] \quad 7 \quad \mathrm{C}_{43} \mathrm{H}_{45} \mathrm{ClGeN}_{3}$}

To a stirred solution of [ $\mathrm{GeCl}_{2}$.dioxane] $(0.23 \mathrm{~g}, 1.0 \mathrm{mmol})$ and dicyclohexylcarbodiimide $(0.21 \mathrm{~g}, 1.0 \mathrm{mmol})$ in THF (6 mL) was added a solution of LiNHAr* $(0.500 \mathrm{~g}, 1.1 \mathrm{mmol})$ in THF $(2 \mathrm{~mL})$ and the resulting solution was left to stir overnight. The solution was diluted with toluene $(2 \mathrm{~mL})$ and the solvent was removed under reduced pressure. The residue was extracted into hexane $(5 \mathrm{~mL})$ and filtered through celite to give a yellow solution. The solution was cooled to $-30^{\circ} \mathrm{C}$ until X-ray quality crystals were grown.

Yield: $402 \mathrm{mg}$ (56\%)

${ }^{1} \mathrm{H} N M R\left(C_{6} \mathrm{D}_{6}, 600 \mathrm{MHz}\right) \delta 7.64\left(\mathrm{~d},, J_{\mathrm{HH}}=7.6 \mathrm{~Hz}, 2 \mathrm{H}\right) ; 7.47\left(\mathrm{~d}, J_{\mathrm{HH}}=8.1 \mathrm{~Hz}, 2 \mathrm{H}\right) ; 7.45\left(\mathrm{~d}, J_{\mathrm{HH}}=2.7 \mathrm{~Hz}, 1 \mathrm{H}\right) ; \mathrm{k}$ $7.38(\mathrm{~s}, 1 \mathrm{H}) ; 7.25\left(\mathrm{~d}, J_{\mathrm{HH}}=7.3 \mathrm{~Hz}, 2 \mathrm{H}\right) ; 7.05\left(\mathrm{t}, \mathrm{J}_{\mathrm{HH}}=6.9 \mathrm{~Hz}, 8 \mathrm{H}\right) ; 6.95(\mathrm{~m}, 2 \mathrm{H}) ; 5.95(\mathrm{~s}, 1 \mathrm{H}) ; 2.01(\mathrm{~m}, 2 \mathrm{H}, \mathrm{Cy}-$ H); $1.87(\mathrm{~m}, 2 \mathrm{H}, \mathrm{Cy}-\mathrm{H}) ; 1.66(\mathrm{~m}, 2 \mathrm{H}, \mathrm{Cy}-\mathrm{H}) ; 1.45(\mathrm{~m}, 2 \mathrm{H}, \mathrm{Cy}-\mathrm{H}) ; 1.32(\mathrm{~m}, 8 \mathrm{H}, \mathrm{Cy}-\mathrm{H}) ; 1.08\left(\mathrm{~s}, 9 \mathrm{H}, \mathrm{C}\left(\mathrm{CH}_{3}\right)_{3}\right)$; $0.81(\mathrm{~m}, 2 \mathrm{H}, \mathrm{Cy}-\mathrm{H}) ; 0.72(\mathrm{~m}, 2 \mathrm{H}, \mathrm{Cy}-\mathrm{H}) ; 0.46$ (m, 1H, ipso-Cy-H); 0.10 (m, 1H, ipso-Cy-H).

Other ${ }^{1} \mathrm{H}$ signals assumed to be hidden under toluene impurity.

${ }^{13} \mathrm{C} \mathrm{NMR}\left(\mathrm{C}_{6} \mathrm{D}_{6}, 125 \mathrm{MHz}\right) \delta 203.7(\mathrm{NCN}) ; 148.4(\operatorname{ArC}) ; 132.7(\operatorname{ArC}) ; 130.5(\operatorname{ArC}) ; 130.3(\operatorname{ArC}) ; 130.2(\operatorname{ArC}) ;$ $129.8(\operatorname{ArC}) ; 129.5$ (ArC); $129.4(\operatorname{ArC}) ; 128.7$ (ArC); 128.6 (ArC); $128.5(\operatorname{ArC}) ; 128.4(\operatorname{ArC}) ; 126.7(\operatorname{ArC}) ; k 35.8$ (CyC); 35.7 (CyC); 33.2 (CyC); $31.3\left(\mathrm{CCH}_{3}\right) ; 30.1$ ( $\left.\mathrm{CPh}_{2}\right)$; 24.8 (CyC); 25.1 (CyC); 25.3 (CyC); 25.6 (CyC); 25.9 (CyC); 26.5 (CyC). Toluene impurity present. Other ${ }^{13} \mathrm{C}$ signals assumed to be hidden by the solvent signal.

\subsubsection{2 [Ge(HMDS) $\left.{ }_{2}(\mathrm{NMes})\right] \quad \mathrm{C}_{21} \mathrm{H}_{47} \mathrm{GeN}_{3} \mathrm{Si}_{4}$}

To a stirred solution of [Ge(HMDS) 2 ] $(140 \mathrm{mg}, 0.36 \mathrm{mmol})$ in hexane $5 \mathrm{~mL}$ at $-30{ }^{\circ} \mathrm{C}$ was added [MesN $\mathrm{N}_{3}$ ( 57 $\mathrm{mg}, 0.35 \mathrm{mmol})$ in hexane $(3 \mathrm{~mL})$. The resulting orange solution was immediately transferred into the freezer at $-30^{\circ} \mathrm{C}$ and $\mathrm{X}$-ray quality crystals were grown overnight.

Yield: $102 \mathrm{mg}, 55 \%$ 
${ }^{1} \mathrm{H}$ NMR $\left(\mathrm{C}_{6} \mathrm{D}_{6}, 600 \mathrm{MHz}\right) \delta 6.99$ (s, 2H, NC 6 -2,6-(Me)-4-(Me)H $\left.\mathrm{H}_{2}\right) ; 2.47$ (s, 6H, NC 6 -2,6-(Me)-4-(Me) $\left.\mathrm{H}_{2}\right) ; 2.28 \mathrm{k}$ (s, 3H, NC $\left.6-2,6-(\mathrm{Me})-4-(\mathrm{Me}) \mathrm{H}_{2}\right), 0.29\left(\mathrm{~s}, 36 \mathrm{H},\left(\mathrm{SiMe}_{3}\right)_{4}\right)$.

${ }^{13} \mathrm{C}$ NMR ( $\left.\mathrm{C}_{6} \mathrm{D}_{6}, 125 \mathrm{MHz}\right) \delta 149.8$ (N-C), 129.3 (ArC), 129.2 (ArC), $20.7\left(\mathrm{NC}_{6}-2,6-(\mathrm{Me})_{2}-4-(\mathrm{Me}) \mathrm{H}_{2}\right) ; 4.5$ $\left.\left(\mathrm{SiMe}_{3}\right)_{4}\right), 4.2\left(\mathrm{NC}_{6}-2,6-(\mathrm{Me})_{2}-4-(\mathrm{Me}) \mathrm{H}_{2}\right)$. The final aromatic signal was assumed to be obscured by the solvent resonance.

\subsubsection{3 $\quad\left[\mathrm{Ge}(\mathrm{HMDS})_{2}\left(\mathrm{~N}_{4} \mathrm{Ph}_{2}\right)\right] \quad 10 \mathrm{C}_{24} \mathrm{H}_{46} \mathrm{GeN}_{6} \mathrm{Si}_{4}$}

To a solution of [Ge(HMDS) $)_{2}$ (100 mg, $\left.0.25 \mathrm{mmol}\right)$ in $\mathrm{C}_{6} \mathrm{D}_{6}(0.15 \mathrm{~mL})$ was added one equivalent of phenylazide $\left[\mathrm{PhN}_{3}\right](30 \mathrm{mg}, 0.25 \mathrm{mmol})$ in $\mathrm{C}_{6} \mathrm{D}_{6}(0.15 \mathrm{~mL})$. The solution was mixed by extracting into and out of a pipette for one minute before the now orange solution was put into an NMR tube and sealed with a Young's tap. The reaction was monitored by ${ }^{1} \mathrm{H}$ NMR spectropscopy until there was no more phenylazide left in solution (10 miuntes). The NMR tube was taken back into the glovebox where toluene $(1 \mathrm{~mL})$ was added and the solvent was removed under reduced pressure. The residue was extracted into hexane $(4 \mathrm{~mL})$ and filtered through celite. The solvent was removed under reduced pressure to give the product as a yellow/orange solid.

Yield: $58 \mathrm{mg}(76 \%)$

${ }^{1} \mathrm{H}$ NMR $\left(\mathrm{C}_{6} \mathrm{D}_{6}, 600 \mathrm{MHz}\right) \delta 7.62\left(\mathrm{~d}, \mathrm{~J}_{\mathrm{HH}}=8.1 \mathrm{~Hz}, 2 \mathrm{H}\right.$, para ArH); 7.00-6.93 (m, 4H, ArH); 6.89-6.80 (m, 4H, $\mathrm{ArH}) ; 0.24\left(\mathrm{~s}, 36 \mathrm{H},\left(\mathrm{N}\left(\mathrm{SiMe}_{3}\right)_{2}\right)_{2}\right)$.

${ }^{13} \mathrm{C}$ NMR $\left(\mathrm{C}_{6} \mathrm{D}_{6}, 125 \mathrm{MHz}\right) \delta 145.7 ; 138.5 ; 130.4 ; 129.3 ; 127.0 ; 125.7 ; 31.6 ; 30.1$.

\subsubsection{4 [Ge(NAPH) $\left.\left(\mathrm{N}_{4} \mathrm{Mes}_{2}\right)\right] \quad 8 \quad \mathrm{C}_{34} \mathrm{H}_{46} \mathrm{GeN}_{6} \mathrm{Si}_{2}$}

To a solution of [Ge(NAPH $\left.{ }^{\mathrm{TMS}}\right)$ ] ( $\left.30 \mathrm{mg}, 0.08 \mathrm{mmol}\right)$ in $\mathrm{C}_{6} \mathrm{D}_{6}(0.20 \mathrm{~mL})$ was added a solution of $\left[\mathrm{MesN}_{3}\right.$ ] in $\mathrm{C}_{6} \mathrm{D}_{6}(0.10 \mathrm{~mL})$. The solution immediately went from orange to burnt orange. The solution was transferred into an NMR tube fitted with a Young's tap and monitored by ${ }^{1} \mathrm{H}$ NMR spectrscopy. When no free azide was observed by ${ }^{1} \mathrm{H}$ NMR spectroscopy (1 minute) the NMR tube was taken into the glove box, diluted with toluene $(1 \mathrm{~mL})$ and the volatiles were removed under reduced pressure to give the compound as an orange solid.

Yield: $22 \mathrm{mg}(87 \%)$. 
${ }^{1} \mathrm{H} \mathrm{NMR}\left(\mathrm{C}_{6} \mathrm{D}_{6}, 600 \mathrm{MHz}\right) \delta \delta 7.19\left(\mathrm{~d}, J_{\mathrm{HH}}=7.9 \mathrm{~Hz}, 2 \mathrm{H}, \mathrm{ArH}\right) ; 7.03\left(\mathrm{t}, J_{\mathrm{HH}}=7.9 \mathrm{~Hz}, 2 \mathrm{H}, \mathrm{ArH}\right) ; 6.87\left(\mathrm{~d}, J_{\mathrm{HH}}=7.9\right.$

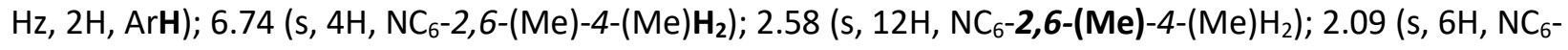
2,6-(Me)-4-(Me) $\left.\mathrm{H}_{2}\right) ; 0.09$ (s, 18H, $\left.\left(\mathrm{SiMe}_{3}\right)_{2}\right)$.

${ }^{13} \mathrm{C}$ NMR $\left(\mathrm{C}_{6} \mathrm{D}_{6}, 125 \mathrm{MHz}\right) \delta 144.0(\operatorname{ArC}) ; 139.0(\operatorname{ArC}) ; 136.8(\operatorname{ArC}) ; 133.6(\operatorname{ArC}) ; 131.9(\operatorname{ArC}) ; 130.6(\operatorname{ArC}) ;$ $125.7(\operatorname{ArC}) ; 121.5(\operatorname{ArC}) ; 116.3(\operatorname{ArC}) ; 112.7(\operatorname{ArC}) ; 22.7\left(\mathrm{NC}_{6}-2,6-(\mathrm{Me})-4-(\mathrm{Me}) \mathrm{H}_{2}\right) ; 20.5$ ( $\mathrm{NC}_{6}-2,6-(\mathrm{Me})-4-$ $\left.(\mathrm{Me}) \mathrm{H}_{2}\right) ; 2.2\left(\left(\mathrm{SiCH}_{3}\right)_{3}\right)$.

\subsubsection{5 [(TMSN)Ge(HMDS)(N(TMS)Ar*)] $11 \mathrm{C}_{48} \mathrm{H}_{69} \mathrm{GeN}_{3} \mathrm{Si}_{4}$}

To a solution of [Ge(HMDS) 2 ( $58 \mathrm{mg}, 0.15 \mathrm{mmol})$ in hexane $(2 \mathrm{~mL})$ was added [ $\left.\mathrm{N}_{3} \mathrm{Ar}^{*}\right](90 \mathrm{mg}, 0.18 \mathrm{mmol})$ at $-30^{\circ} \mathrm{C}$. The solution was heated up to room temperature were and orange/yellow solid precipitated over an hour. The suspension was heated to supersaturate the solution. The solution was cooled to -30 ${ }^{\circ} \mathrm{C}$ to give crystals suitable for $\mathrm{X}$-ray diffraction overnight.

${ }^{1} \mathrm{H}$ NMR $\left(\mathrm{C}_{6} \mathrm{D}_{6}, 600 \mathrm{MHz}\right) \delta 7.66\left(\mathrm{~d}, J_{\mathrm{HH}}=7.2 \mathrm{~Hz}, 4 \mathrm{H}, \operatorname{ArH}\right) ; 7.51\left(\mathrm{~d}, J_{\mathrm{HH}}=7.0 \mathrm{~Hz}, 4 \mathrm{H}, \operatorname{ArH}\right) ; 7.26\left(\mathrm{t}, J_{\mathrm{HH}}=7.5\right.$ $\mathrm{Hz}, 4 \mathrm{H}, \mathrm{ArH}) ; 6.35\left(\mathrm{~s}, 2 \mathrm{H},\left(\mathrm{CHPh}_{2}\right)_{2}\right) ; 1.04\left(\mathrm{~s}, 9 \mathrm{H}, \mathrm{C}\left(\mathrm{CH}_{3}\right)_{3}\right) ; 0.49$ (s, 9H, SiMe $) ; 0.42\left(\mathrm{~s}, 18 \mathrm{H}, \mathrm{N}\left(\mathrm{SiMe}_{3}\right)_{2}\right) ;-0.15$ (s, 9H, $\left.\mathrm{SiMe}_{3}\right)$.

Other aromatic proton resonances are obscured by $[\mathrm{Ar} * \mathrm{Cl}](26)$ and assumed $\left[(\mathrm{HMDS})_{2} \mathrm{Ge}\left(\mathrm{NAr}^{*}\right)\right]$ impurities in the NMR. ${ }^{13} \mathrm{C}$ NMR data was not obtained for this compound.

\subsubsection{6 [(HMDS $\left.)_{2} \mathrm{Ge}\left(\mathrm{N}_{2} \mathrm{OPhNC}(\mathrm{O}) \mathrm{NMes}\right)\right] 13 \mathrm{C}_{28} \mathrm{H}_{51} \mathrm{GeN}_{5} \mathrm{O}_{3} \mathrm{Si}_{4}$}

In an NMR tube fitted with a Young's tap, [Ge(HMDS) 2 ] $(70 \mathrm{mg}, 0.18 \mathrm{mmol})$, [MesN $\mathrm{M}_{3}$ (29 mg, $\left.0.18 \mathrm{mmol}\right)$ and 4-nitrophenylisocyanate $(29 \mathrm{mg}, 0.18 \mathrm{mmol})$ were added together in $\mathrm{C}_{6} \mathrm{D}_{6}(0.30 \mathrm{~mL})$. The solution was monitored by ${ }^{1} \mathrm{H}$ NMR spectroscopy until no free azide was observed (20 hours). The NMR tube was taken back into a nitrogen glovebox where toluene $(1 \mathrm{~mL})$ was added before the solvent was evaporated under reduced pressure. The residue was extracted into hexane $(2 \mathrm{~mL})$, filtered through celite and reduced to dryness to give an off-white solid.

Yield: $72 \mathrm{mg}$ (59\%).

${ }^{1} \mathrm{H}$ NMR $\left(\mathrm{C}_{6} \mathrm{D}_{6}, 600 \mathrm{MHz}\right) \delta 8.07\left(\mathrm{~d}, J_{\mathrm{HH}}=6.4 \mathrm{~Hz}, 2 \mathrm{H}\right.$, meta- $\left.\mathrm{C}_{6} \mathrm{H}_{4} \mathrm{NO}_{2}\right) ; 7.64\left(\mathrm{~d}, J_{\mathrm{HH}}=8.1 \mathrm{~Hz}, 2 \mathrm{H}\right.$, ortho$\left.\mathrm{C}_{6} \mathrm{H}_{4} \mathrm{NO}_{2}\right) ; 6.80\left(\mathrm{~s}, 2 \mathrm{H}, \mathrm{NC}_{6}-2,6-(\mathrm{Me})_{2}-4-(\mathrm{Me}) \mathrm{H}_{2}\right) ; 2.43$ (s, 6H, NC 6 -2,6-(Me)-4-(Me) $\left.\mathrm{H}_{2}\right) ; 2.13$ (s, 3H NC${ }_{6}-2,6-$ (Me)-4-(Me) $\left.\mathrm{H}_{2}\right) ; 0.20\left(\mathrm{~s}, 36 \mathrm{H},\left(\mathrm{N}(\mathrm{TMS})_{3}\right)_{2}\right)$. 
${ }^{13} \mathrm{C}$ NMR $\left(\mathrm{C}_{6} \mathrm{D}_{6}, 125 \mathrm{MHz}\right) \delta 142.8(\mathrm{C}=0) ; 135.8(\operatorname{ArC}) ; 129.9(\operatorname{ArC}) ; 128.6(\operatorname{ArC}) ; 124.7(\operatorname{ArC}) ; 121.6(\operatorname{ArC}) ;$ $32.0(\mathrm{MeC}), 21.7(\mathrm{MeC}), 5.8(\mathrm{TMS})$. Other ${ }^{13} \mathrm{C}$ signals assumed to be hidden under the solvent signal.

Anal. Calcd. for $\mathrm{C}_{28} \mathrm{H}_{51} \mathrm{GeN}_{5} \mathrm{O}_{3} \mathrm{Si}_{4}$ (691.23): C, 48.69; $\mathrm{H}, 7.44 ; \mathrm{N}, 10.14$; Found: $\mathrm{C}, 48.78 ; \mathrm{N}, 7.39 ; \mathrm{H}, 9.96$.

\subsubsection{7 [(HMDS $\left.)_{2} \mathrm{Ge}(\mathrm{CCPh})(\mathrm{NHPh})\right] \quad 12 \quad \mathrm{C}_{29} \mathrm{H}_{53} \mathrm{GeN}_{3} \mathrm{Si}_{4}$}

To a stirred solution of $\left[\mathrm{Ge}(\mathrm{HMDS})_{2}\right](60 \mathrm{mg}, 0.16 \mathrm{mmol})$ in hexane $(6 \mathrm{~mL})$ was added dropwise a solution of [Mes $\mathrm{N}_{3}$ ] (25 mg, $\left.0.16 \mathrm{mmol}\right)$ in hexane $(2 \mathrm{~mL})$. A colourless gas was released and the solution turned from orange to yellow. After 10 minutes a suspension of phenylacetylene (16 $\mathrm{mg}, 0.16 \mathrm{mmol}$ ) in hexane $(2 \mathrm{~mL})$ was added dropwise. The reaction was left to stir overnight before being concentrated under reduced pressure and cooled to $-30{ }^{\circ} \mathrm{C}$ to grow crystals suitable for single crystal X-ray diffraction.

Yield: $68 \mathrm{mg}$, (67\%).

${ }^{1} \mathrm{H}$ NMR $\left(\mathrm{C}_{6} \mathrm{D}_{6}, 600 \mathrm{MHz}\right) \delta 7.61\left(\mathrm{~m}, 1 \mathrm{H}\right.$, para-ArH) $7.43(\mathrm{~m}, 2 \mathrm{H}, \mathrm{ArH}) ; 6.93(\mathrm{~m}, 2 \mathrm{H}, \mathrm{ArH}) ; 6.82\left(\mathrm{~s}, 2 \mathrm{H}, \mathrm{NC}_{6^{-}}\right.$ 2,6-(Me)-4-(Me)H $\mathrm{H}_{2}$ ); 3.11 (br s, 1H, NH); 2.53 (s, 6H, NC6-2,6-(Me)-4-(Me)H2); 2.17 (s, 3H, NC 6-2,6-(Me)-4$\left.\left.(\mathrm{Me}) \mathrm{H}_{2}\right) ; 0.47\left(\mathrm{~s}, 36 \mathrm{H},\left(\mathrm{N}\left(\mathrm{SiMe}_{3}\right)_{2}\right)_{2}\right)\right)$.

${ }^{13} \mathrm{C}$ NMR $\left(\mathrm{C}_{6} \mathrm{D}_{6}, 125 \mathrm{MHz}\right) \delta 163.6(\mathrm{ArC}) ; 141.5(\mathrm{ArC}) ; 131.6(\operatorname{ArC}) ; 130.1(\operatorname{ArC}) ; 130.0(\operatorname{ArC}) ; 129.6(\operatorname{ArC}) ;$

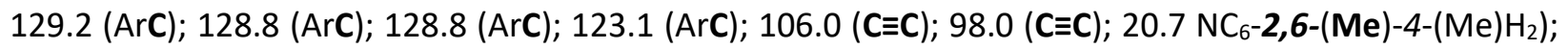
$\left.\left.6.4\left(\mathrm{~N}\left(\mathrm{SiMe}_{3}\right)_{2}\right)_{2}\right)\right) ; 3.7\left(\mathrm{NC}_{6}-2,6-(\mathrm{Me})-4-(\mathrm{Me}) \mathrm{H}_{2}\right)$.

\subsubsection{8 $\quad\left[\mathrm{Cp}_{2} \mathrm{Zr}(\mathrm{Bn})(\mathrm{NHPh})\right] \quad 17 \mathrm{C}_{23} \mathrm{H}_{23} \mathrm{NZr}$}

To a solution of $\left[\mathrm{Bn}_{2} \mathrm{ZrCp}_{2}\right](75 \mathrm{mg}, 0.19 \mathrm{mmol})$ in $\mathrm{C}_{6} \mathrm{D}_{6}(0.20 \mathrm{~mL})$ was added a solution of aniline (17 mg, $0.18 \mathrm{mmol})$ in $\mathrm{C}_{6} \mathrm{D}_{6}(0.20 \mathrm{~mL})$. The resulting orange solution was transferred into an NMR tube fitted with a Young's tap and heated to $100^{\circ} \mathrm{C}$ for two days using an aluminium heating block to give a yellow solution and a green solid (18). The NMR tube was taken back into the glovebox and the yellow solution was the green solid was decantered from the solution. Toluene was added $(2 \mathrm{~mL})$ to the solution before the solvent was removed under reduced pressure. The residue was extracted into hexane $(3 \mathrm{~mL})$, filtered through celite and placed in the freezer at $-30{ }^{\circ} \mathrm{C}$ from which yellow plates suitable for X-ray diffraction were grown. Yield: 52 mg, 69\%. 
${ }^{1} \mathrm{H} \mathrm{NMR}\left(\mathrm{C}_{6} \mathrm{D}_{6}, 600 \mathrm{MHz}\right)$ 8 7.26-7.19 (m, 4H, ArH); 7.06 (d, $\left.J_{\mathrm{HH}}=7.8 \mathrm{~Hz}, 2 \mathrm{H}, \mathrm{ArH}\right) ; 6.90-6.86(\mathrm{~m}, 2 \mathrm{H}, \operatorname{ArH})$; $6.76\left(\mathrm{~d}, \mathrm{~J}_{\mathrm{HH}}=7.6 \mathrm{~Hz}, 2 \mathrm{H}, \mathrm{ArH}\right) ; 5.51\left(\mathrm{~s}, 10 \mathrm{H}, \mathrm{C}_{5} \mathrm{H}_{5}\right) ; 3.58(\mathrm{br} \mathrm{s}, 1 \mathrm{H}, \mathrm{NH}) ; 2.24\left(\mathrm{~s}, 2 \mathrm{H}, \mathrm{CH}_{2} \mathrm{Ph}\right)$.

${ }^{13} \mathrm{C} \mathrm{NMR}\left(\mathrm{C}_{6} \mathrm{D}_{6}, 125 \mathrm{MHz}\right) \delta 156.1(\operatorname{ArC}), 153.9(\operatorname{ArC}), 129.0(\operatorname{ArC}), 128.3(\operatorname{ArC}), 126.1(\operatorname{ArC}), 121.0(\operatorname{ArC})$, $120.4(\operatorname{ArC}), 120.2(\operatorname{ArC}), 110.7\left(\mathrm{C}_{5} \mathrm{H}_{5}\right), 50.0\left(\mathrm{CH}_{2} \mathrm{C}_{6} \mathrm{H}_{5}\right)$.

\subsubsection{9 $\quad\left[\left(\mathrm{Cp}_{2} \mathrm{Zr}(\mu-\mathrm{NPh})\right)_{2}\right] \quad 18 \quad \mathrm{C}_{32} \mathrm{H}_{30} \mathrm{~N}_{2} \mathrm{Zr}_{2}$}

To a solution of $\left[\mathrm{Bn}_{2} \mathrm{ZrCp}_{2}\right](75 \mathrm{mg}, 0.19 \mathrm{mmol})$ in $\mathrm{C}_{6} \mathrm{D}_{6}(0.20 \mathrm{~mL})$ was added a solution of aniline $(17 \mathrm{mg}$, $0.18 \mathrm{mmol})$ in $\mathrm{C}_{6} \mathrm{D}_{6}(0.20 \mathrm{~mL})$. The resulting orange solution was transferred into an NMR tube fitted with a Young's tap and heated to $100{ }^{\circ} \mathrm{C}$ for two days to give a yellow solution and a green solid (18). The green solid was decanted from the solution and washed with THF $(2 \times 2 \mathrm{~mL})$. The crystals were insoluble in all available NMR solvents (D6-benzene, D8-THF, D3-acetonitrile, heavy water, D6-DMSO and D8-toluene). However, the product was identified by single crystal X-ray diffraction.

\subsubsection{0 $\quad\left[\mathrm{Cp}_{2} \mathrm{Zr}(\mathrm{Bu})\left(\mathrm{N}_{3}(\mathrm{Bu})(\mathrm{Mes})\right)\right] 21 \mathrm{C}_{27} \mathrm{H}_{39} \mathrm{~N}_{3} \mathrm{Zr}$}

To a stirred solution on $\left[\mathrm{Cp}_{2} \mathrm{ZrCl}_{2}\right](0.29 \mathrm{~g}, 1.0 \mathrm{mmol})$ in THF $(5 \mathrm{~mL})$ at $-30{ }^{\circ} \mathrm{C}$ was added dropwise a $2 \mathrm{M}$ solution $n$-BuLi in cyclohexane $(1.0 \mathrm{~mL}, 2.0 \mathrm{mmol})$, resulting in a yellow solution. After letting this solution come up to room temperature over 15 minutes a solution of [Mes $\left.\mathrm{N}_{3}\right](0.17 \mathrm{~g}, 1.0 \mathrm{mmol})$ in hexane was added to the solution dropwise. After 1.5 hours the dark orange solution had the solvent removed under reduced pressure to give a dark oily residue. This oil was redissolved in a minimum volume of diethyl ether $(2 \mathrm{~mL})$ and cooled to $-30{ }^{\circ} \mathrm{C}$ to grow $\mathrm{X}$-ray quality crystals.

Yield: $352 \mathrm{mg}$ (71\%)

${ }^{1} \mathrm{H} \mathrm{NMR}\left(\mathrm{C}_{6} \mathrm{D}_{6}, 600 \mathrm{MHz}\right) \delta 6.81$ (s, 2H, NC 6 -2,6-(Me) $\left.{ }_{2}-4-(\mathrm{Me}) \mathrm{H}_{2}\right) ; 5.79\left(\mathrm{~s}, 10 \mathrm{H}, \mathrm{C}_{5} \mathrm{H}_{5}\right) ; 3.26\left(\mathrm{t}, \mathrm{J}_{\mathrm{HH}}=6.1 \mathrm{~Hz}\right.$, $2 \mathrm{H}, \mathrm{Bu}), 2.33\left(\mathrm{~s}, 6 \mathrm{H}, \mathrm{NC}_{6}-2,6-(\mathrm{Me})-4-\left(\mathrm{Me}_{2} \mathrm{H}_{2}\right) ; 2.15\right.$ (s, 3H, NC 6 -2,6-(Me)-4-(Me) $\left.\mathrm{H}_{2}\right) ; 1.67-1.62$ (m, 2H, Bu); $1.31\left(\mathrm{sex}, J_{\mathrm{HH}}=7.6 \mathrm{~Hz}, 2 \mathrm{H}, \mathrm{Bu}\right) ; 1.18-1.11(\mathrm{~m}, 5 \mathrm{H}, \mathrm{Bu}) ; 0.86\left(\mathrm{t}, J_{\mathrm{HH}}=7.1 \mathrm{~Hz}, 3 \mathrm{H}, \mathrm{Bu}\right) ; 0.33-0.30(\mathrm{~m}, 2 \mathrm{H}, \mathrm{Bu})$.

${ }^{13} \mathrm{C} \mathrm{NMR}\left(\mathrm{C}_{6} \mathrm{D}_{6}, 125 \mathrm{MHz}\right) \delta 144.8(\mathrm{ArC}), 134.0(\operatorname{ArC}), 133.9(\operatorname{ArC}), 128.3(\operatorname{ArC}), 109.3\left(\mathrm{C}_{5} \mathrm{H}_{5}\right), 67.0(\mathrm{BuC}), 55.8$ (BuC), 48.0 (BuC), 36.8 (BuC), 32.6 (BuC), 30.3 (BuC), 20.9 (BuC), 19.7 (ortho -Me), 15.6 (para-Me), 14.1 (BuC). 


\subsubsection{1 $\left[\mathrm{Cp}_{2} \mathrm{Zr}(\mathrm{Me})\left(\mathrm{N}_{3}(\mathrm{Me})(\mathrm{Mes})\right)\right] 22 \quad \mathrm{C}_{21} \mathrm{H}_{27} \mathrm{~N}_{3} \mathrm{Zr}$}

To a stirred solution on $\left[\mathrm{Cp}_{2} \mathrm{ZrMe}\right.$ ] $(93 \mathrm{mg}, 0.32 \mathrm{mmol})$ in toluene $(5 \mathrm{~mL})$ at $-30{ }^{\circ} \mathrm{C}$ was added a solution of $\left[\mathrm{MesN}_{3}\right](0.17 \mathrm{~g}, 1.0 \mathrm{mmol})$ in toluene $(2 \mathrm{~mL})$ dropwise. The solution was left to stir at room temperature overnight. The solvent was reduced to approximately $1.5 \mathrm{~mL}$ before being placed in the freezer at $-30^{\circ} \mathrm{C}$ overnight to give the product as crystals.

Yield $0.11 \mathrm{~g}(85 \%)$.

${ }^{1} \mathrm{H} \mathrm{NMR}\left(\mathrm{C}_{6} \mathrm{D}_{6}, 600 \mathrm{MHz}\right) \delta 6.82\left(\mathrm{~s}, 2 \mathrm{H},\left(\mathrm{N}-2,4,6-\mathrm{MeC}_{6} \mathrm{H}_{2}\right)\right) ; 5.69\left(\mathrm{~s}, 10 \mathrm{H}, \mathrm{C}_{5} \mathrm{H}_{5}\right), 3.00(\mathrm{~s}, 3 \mathrm{H}$, (NMe)); $2.33(\mathrm{~s}$, $\left.6 \mathrm{H},\left(\mathrm{N}-2,4,6-\mathrm{MeC}_{6} \mathrm{H}_{2}\right)\right) ; 2.17\left(\mathrm{~s}, 3 \mathrm{H},\left(\mathrm{N}-2,4,6-\mathrm{MeC}_{6} \mathrm{H}_{2}\right)\right) ;-0.23(\mathrm{~s}, 3 \mathrm{H},(\mathrm{ZrMe}))$.

${ }^{13} \mathrm{C}$ NMR $\left(\mathrm{C}_{6} \mathrm{D}_{6}, 125 \mathrm{MHz}\right) \delta 144.8(\mathrm{ArC}), 134.2(\operatorname{ArC}), 133.6(\mathrm{ArC}), 129.4(\operatorname{ArC}), 109.1\left(\mathrm{C}_{5} \mathrm{H}_{5}\right), 43.0(\mathrm{NMe})$, $28.3(\mathrm{ZrMe}), 21.0\left(\mathrm{~N}-2,4,6-\mathrm{MeC}_{6} \mathrm{H}_{2}\right), 19.6\left(\mathrm{~N}-2,4,6-\mathrm{MeC}_{6} \mathrm{H}_{2}\right)$.

\subsubsection{2 $\quad\left[\mathrm{Cp}_{2} \mathrm{Zr}(\mathrm{Me})\left(\mathrm{N}_{3}(\mathrm{Me})\left(\mathrm{C}_{6} \mathrm{H}_{5}\right)\right] \quad 23 \quad \mathrm{C}_{18} \mathrm{H}_{21} \mathrm{~N}_{3} \mathrm{Zr}\right.$}

To a stirred solution on $\left[\mathrm{Cp}_{2} \mathrm{ZrMe} \mathrm{H}_{2}\right](0.67 \mathrm{~g}, 2.66 \mathrm{mmol})$ in diethyl ether $(5 \mathrm{~mL})$ at $-30{ }^{\circ} \mathrm{C}$ was added a solution of $\left[\mathrm{PhN}_{3}\right](0.32 \mathrm{~g}, 2.66 \mathrm{mmol})$ in diethyl ether $(2 \mathrm{~mL})$ dropwise. The solution was left to for 5 days at room temperature. The solvent was removed under reduced pressure. The residue was extracted into hexane $(2 \mathrm{~mL})$. The resulting suspension was flitered through celite, concentrated to $1 \mathrm{~mL}$ and cooled to $30{ }^{\circ} \mathrm{C}$ overnight to give the product as colourless crystals.

${ }^{1} \mathrm{H}$ NMR $\left(\mathrm{C}_{6} \mathrm{D}_{6}, 600 \mathrm{MHz}\right)$ ठ 7.33-7.28 (m, 4H, ortho, meta ArH); $7.01\left(\mathrm{~m}, 1 \mathrm{H}\right.$, para-ArH); $5.60\left(\mathrm{~s}, 10 \mathrm{H}, \mathrm{C}_{5} \mathrm{H}_{5}\right)$; 3.14 (s, 3H, NMe); -0.04 (s, 3H, ZeMe).

${ }^{13} \mathrm{C} \mathrm{NMR}\left(\mathrm{C}_{6} \mathrm{D}_{6}, 125 \mathrm{MHz}\right) \delta 150.1$ (ArC), $129.4(\operatorname{ArC}), 122.5(\mathrm{ArC}), 115.9(\operatorname{ArC}), 109.1\left(\mathrm{C}_{5} \mathrm{H}_{5}\right), 42.4(\mathrm{NMe})$, 28.5 (ZrMe).

Anal. Calcd. for $\mathrm{C}_{18} \mathrm{H}_{21} \mathrm{~N}_{3} \mathrm{Zr}$ (369.08): C, 58.34; H, 5.71; N, 11.34. Found: C, 58.26; H, 5.90; N, 11.37 .

IR (Nujol, cm¹); 3093 (w), 2950 (s Nujol), 2920 (s, Nujol), 2850 (s, Nujol), 2729 (w), 1590 (w), 1464 (s, Nujol), 1380 (s, Nujol), 1290 (m), 1166 (w), 1020 (w), 802 (m), 756 (w), 725 (w), 694 (w), $520(w)$.

\subsubsection{3 $\quad\left[\mathrm{Cp}_{2} \mathrm{Zr}(\mathrm{Bn})\left(\mathrm{N}_{3}(\mathrm{Bn})(\mathrm{Mes})\right)\right] 24 \mathrm{C}_{33} \mathrm{H}_{35} \mathrm{~N}_{3} \mathrm{Zr}$}

To a stirred solution of $\left[\mathrm{Cp}_{2} \mathrm{ZrBn}_{2}\right](180 \mathrm{mg}, 0.45 \mathrm{mmol})$ in hexane $(3 \mathrm{~mL})$ at $-30{ }^{\circ} \mathrm{C}$ was added a solution of [Mes $\mathrm{N}_{3}$ ] (53 mg, $\left.0.45 \mathrm{mmol}\right)$ in hexane $(2 \mathrm{~mL})$ dropwise. The reaction was left to stir for 24 hours. The 
solution was filtered through celite and reduced to $1 \mathrm{~mL}$ volume before being placed in the freezer at -30 ${ }^{\circ} \mathrm{C}$ from which X-ray quality crystals were grown.

Yield: $147 \mathrm{mg}(58 \%)$

${ }^{1} \mathrm{H}$ NMR $\left(\mathrm{C}_{6} \mathrm{D}_{6}, 600 \mathrm{MHz}\right) \delta 7.25\left(\mathrm{~d}, \mathrm{~J}_{\mathrm{HH}}=6.9 \mathrm{~Hz}, 2 \mathrm{H}, \mathrm{ArH}\right) ; 7.14-7.12(\mathrm{~m}, 4 \mathrm{H}, \mathrm{ArH}) ; 7.09\left(\mathrm{t}, \mathrm{J}_{\mathrm{HH}}=7.9 \mathrm{~Hz}, 1 \mathrm{H}\right.$, ArH); $6.99\left(\mathrm{~d}, J_{\mathrm{HH}}=7.7 \mathrm{~Hz}, 2 \mathrm{H}, \mathrm{ArH}\right) ; 6.85\left(\mathrm{t}, J_{\mathrm{HH}}=7.1,1 \mathrm{H}, \mathrm{ArH}\right) ; 6.80(\mathrm{~s}, 2 \mathrm{H}, \mathrm{ArH}) ; 5.62(\mathrm{~s}, 10 \mathrm{H}) ; 4.43(\mathrm{~s}, 2 \mathrm{H}$ $\mathrm{NCH}_{2} \mathrm{Ph}$ ); 2.33 (s, 6H, 2,4,6- $\left.\mathrm{MeC}_{6} \mathrm{H}_{2}\right) ; 2.14\left(\mathrm{~s}, 3 \mathrm{H}, 2,4,6-\mathrm{MeC}_{6} \mathrm{H}_{2}\right) ; 1.70$ (s, 2H, $\mathrm{ZrCH}_{2} \mathrm{Ph}$ ).

${ }^{13} \mathrm{C}$ NMR $\left(\mathrm{C}_{6} \mathrm{D}_{6}, 125 \mathrm{MHz}\right) \delta 153.5(\operatorname{ArC}), 144.1(\operatorname{ArC}), 139.7(\operatorname{ArC}), 135.0(\operatorname{ArC}), 134.0(\operatorname{ArC}), 129.6(\operatorname{ArC})$, $129.0(\mathrm{ArC}), 129.0(\mathrm{ArC}), 128.0(\mathrm{ArC}), 126.3(\mathrm{ArC}), 120.7(\mathrm{ArC}), 110.6\left(\mathrm{C}_{5} \mathrm{H}_{5}\right), 61.7\left(\mathrm{CH}_{2} \mathrm{Ph}\right), 54.0\left(\mathrm{CH}_{2} \mathrm{Ph}\right)$, $21.0\left(\mathrm{~N}-2,4,6-\mathrm{MeC}_{6} \mathrm{H}_{2}\right), 19.5\left(\mathrm{~N}-2,4,6-\mathrm{MeC}_{6} \mathrm{H}_{2}\right)$.

\subsubsection{4 $\quad\left[\mathrm{Cp}_{2} \mathrm{Zr}(\mathrm{NHDipp})(\mathrm{Me})\right] \quad 14 \quad \mathrm{C}_{23} \mathrm{H}_{31} \mathrm{NZr}$}

To a stirred solution of $\left[\mathrm{Cp}_{2} \mathrm{Zr}(\mathrm{Cl})(\mathrm{Me})\right](110 \mathrm{mg}, 0.42 \mathrm{mmol})$ in diethyl ether $(5 \mathrm{~mL})$ at $-30{ }^{\circ} \mathrm{C}$ was added [LiNHDipp] (77 mg, $0.42 \mathrm{mmol})$ in diethyl ether $(5 \mathrm{~mL})$. Upon addition of the lithiated 2,6 diisopropylaniline the solution went yellow and a white solid precipitated. The suspension mixture was left to stir at room temperature overnight. The suspension was then left to settle and the yellow solution was decanted from the white solid and filtered through celite to give a clear yellow solution. The solvent was removed under reduced pressure and the resulting residue was extracted into toluene $(3 \times 5 \mathrm{~mL})$. The extracted solution was filtered through celite and concentrated under reduced pressure to $2 \mathrm{~mL}$. The vial was cooled to -30 ${ }^{\circ} \mathrm{C}$ to give the product as yellow crystals after 2 days.

Yield: $112 \mathrm{mg}(64 \%)$

${ }^{1} \mathrm{H} \mathrm{NMR}\left(\mathrm{C}_{6} \mathrm{D}_{6}, 600 \mathrm{MHz}\right) \delta 7.16\left(\mathrm{~d}, 2 \mathrm{H}, m-\mathrm{C}_{6} \mathrm{H}_{3}\right) ; 7.11\left(\mathrm{t}, 1 \mathrm{H}, p-\mathrm{C}_{6} \mathrm{H}_{3}\right) ; 6.22(\mathrm{br} \mathrm{s}, 1 \mathrm{H}, \mathrm{NH}) ; 5.62\left(\mathrm{~s}, 10 \mathrm{H}, \mathrm{C}_{5} \mathrm{H}_{5}\right)$; $3.41\left(\mathrm{~m}, 2 \mathrm{H}, \mathrm{CH}\left(\mathrm{CH}_{3}\right)_{2}\right) ; 1.24\left(\mathrm{~d}, \mathrm{~J}_{\mathrm{HH}}=6.5 \mathrm{~Hz}, 12 \mathrm{H}, \mathrm{CH}\left(\mathrm{CH}_{3}\right)_{2}\right) ; 0.23\left(\mathrm{~s}, 3 \mathrm{H}, \mathrm{ZrCH}_{3}\right)$.

${ }^{13} \mathrm{C} \mathrm{NMR}\left(\mathrm{C}_{6} \mathrm{D}_{6}, 125 \mathrm{MHz}\right) \delta 152.3(\mathrm{ArC}) ; 126.0(\operatorname{ArC}) ; 123.7(\operatorname{ArC}) ; 123.0(\operatorname{ArC}) ; 110.0\left(\mathrm{C}_{5} \mathrm{H}_{5}\right) ; 28.2\left(\left(\mathrm{CH}_{3}\right)_{2} \mathrm{CH}\right)$; $23.5\left(\left(\mathrm{CH}_{3}\right)_{2} \mathrm{CH}\right) ; 18.9$ ( $\left.\mathrm{ZrMe}\right)$.

\subsubsection{5 $\quad\left[\mathrm{Cp}_{2} \mathrm{Zr}(\mathrm{Me})\left(\mathrm{NHAr}^{*}\right)\right] \quad 15 \quad \mathrm{C}_{47} \mathrm{H}_{47} \mathrm{NZr}$}

To a stirred solution of $\left[\mathrm{Cp}_{2} \mathrm{Zr}(\mathrm{Me})(\mathrm{Cl})\right](270 \mathrm{mg}, 0.99 \mathrm{mmol})$ in diethyl ether $(5 \mathrm{~mL})$ at $-30{ }^{\circ} \mathrm{C}$ was added [LiNHAr*] $(480 \mathrm{mg}, 1.0 \mathrm{mmol})$ in diethyl ether $(5 \mathrm{~mL})$ dropwise over 1 minute. The addition of the red [LiNHAr*] to the colourless solution of $\left[\mathrm{Cp}_{2} \mathrm{Zr}(\mathrm{Me})(\mathrm{Cl})\right]$ resulted in a green solution developing over 20 minutes. The reaction was left to stir overnight before being filtered through celite. The resulting green 
solution was concentrated to dryness under reduced pressure then extracted into toluene $(2 \times 2 \mathrm{~mL})$. The toluene solution was filtered through celite, layered with hexane and cooled to $-30^{\circ} \mathrm{C}$ to give the product as green crystals.

${ }^{1} \mathrm{H}$ NMR $\left(\mathrm{C}_{6} \mathrm{D}_{6}, 600 \mathrm{MHz}\right) \delta 7.24$ (br s, 8H, ArH); 7.19 (m, 8H, ArH); 7.06-6.99 (m, 6H, ArH); 6.01 (br s, 2H, $\left.\left(\mathrm{CHPh}_{2}\right)_{2}\right) ; 5.58\left(\mathrm{~s}, 10 \mathrm{H}, \mathrm{C}_{5} \mathrm{H}_{5}\right) ; 2.79$ (br s, $\left.1 \mathrm{H}, \mathrm{NH}\right) ; 1.09$ (s, 9H. $\left.\mathrm{C}_{6} \mathrm{H}_{2}{ }^{t} \mathrm{Bu}\right) ; 0.30$ (s, 3H, ZrMe).

${ }^{13} \mathrm{C}$ NMR $\left(\mathrm{C}_{6} \mathrm{D}_{6}, 125 \mathrm{MHz}\right) \delta 152.63(\operatorname{ArC}) ; 145.0(\operatorname{ArC}) ; 130.0(\operatorname{ArC}) ; 128.6(\operatorname{ArC}) ; 126.5(\operatorname{ArC}) ; 125.7(\operatorname{ArC}) ;$ $112.8(\mathrm{ArC}) ; 110.0\left(\mathrm{C}_{5} \mathrm{H}_{5}\right) ; 34.5\left(\mathrm{CHPh}_{2}\right) ; 31.5\left(\mathrm{C}_{6} \mathrm{H}_{2} \mathrm{C}\left(\mathrm{CH}_{3}\right)_{3}\right) ; 31.5\left(\mathrm{C}_{6} \mathrm{H}_{2} \mathrm{C}\left(\mathrm{CH}_{3}\right)_{3}\right) ; 21.0\left(\mathrm{ZrCH}_{3}\right)$.

\subsubsection{6 [Cp $2 \mathrm{Zr}(\mathrm{Me})(\mathrm{NHDMP})] \quad 16 \quad \mathrm{C}_{19} \mathrm{H}_{23} \mathrm{NZr}$}

\subsubsection{Method A:}

To a solution of [ $\mathrm{Cp}_{2} \mathrm{ZrMe}$ ] $(100 \mathrm{mg}, 0.40 \mathrm{mmol})$ in toluene $(10 \mathrm{~mL})$ was added 2,6-Dimethylaniline $(48 \mathrm{mg}$, $0.40 \mathrm{mmol}$ ). The solution was transferred to an ampule fitted with a Young's tap and heated using an oil bath at $110^{\circ} \mathrm{C}$ for 4 days. The solvent was removed under reduced pressure and the residue was extracted into hexane and filtered through celite. The solution was layered with toluene $(1 \mathrm{~mL})$ and cooled to $-30{ }^{\circ} \mathrm{C}$ to give crystals the product as orange crystals.

\subsubsection{Method B:}

To a stirred solution of [ $\left.\mathrm{Cp}_{2} \mathrm{Zr}(\mathrm{Me})(\mathrm{Cl})\right]((490 \mathrm{mg}, 1.82 \mathrm{mmol})$ in ether $(10 \mathrm{~mL})$ was added [LiNHDMP] (230 $\mathrm{mg}, 1.82 \mathrm{mmol})$, the reaction was left to stir overnight. The suspension was filtered through celite to before having the solvent removed under reduced pressure. The residue was extracted into toluene $(2 \mathrm{x}$ $5 \mathrm{~mL}$ ) filtered through celite and reduced to dryness under reduced pressure to give the compound as an orange solid.

${ }^{1} \mathrm{H}$ NMR $\left(\mathrm{C}_{6} \mathrm{D}_{6}, 600 \mathrm{MHz}\right)$ 8 7.11-7.07 (m, 3H); 5.34 (s, 10H, $\left.\mathrm{C}_{5} \mathrm{H}_{5}\right) ; 2.04$ (s, 6H, NPhMe $)$; 0.54 (s, 3H, ZrMe). Toluene is also present in the ${ }^{1} \mathrm{H}$ and ${ }^{13} \mathrm{C}$ NMR spectral data.

${ }^{13} \mathrm{C}$ NMR $\left(\mathrm{C}_{6} \mathrm{D}_{6}, 125 \mathrm{MHz}\right) \delta 149.1(\operatorname{ArC}), 128.8(\operatorname{ArC}), 128.8(\operatorname{ArC}), 128.2(\operatorname{ArC}), 110.4\left(\mathrm{C}_{5} \mathrm{H}_{5}\right), 52.5$ (ortho$\left.\mathrm{CH}_{3}\right), 13.9$ (ZrMe).

Anal. Calcd. for $\mathrm{C}_{19} \mathrm{H}_{23} \mathrm{NZr}$ (355.09): C, 63.99; $\mathrm{H}, 6.50 ; \mathrm{N}, 3.93$; Found: $\mathrm{C}, 61.26 ; \mathrm{N}, 3.86 ; \mathrm{H}, 6.49$. 


\subsubsection{7 [Cp $2 \mathrm{Zr}(\mathrm{NAr} *)(\mathrm{THF})] \quad 20 \quad \mathrm{C}_{50} \mathrm{H}_{50} \mathrm{NOZr}$}

In a NMR tube fitted with a young's tap was added [ $\left.\mathrm{Cp}_{2} \mathrm{Zr}(\mathrm{Me})\left(\mathrm{NHAr}^{*}\right)\right](60 \mathrm{mg}, 0.08 \mathrm{mmol})$ in $\mathrm{C}_{6} \mathrm{D}_{6}(0.30$ $\mathrm{mL}$ ) and 5 drops of THF. The NMR tube was sealed and heated on an aluminium heating block for 5 days at $100^{\circ} \mathrm{C}$ (the author notes this is above the boiling point of deuterated benzene, hence caution is advised) The NMR tube was taken back into the glovebox where hexane $(1 \mathrm{~mL})$ was added and the solvent was removed under reduced pressure. The residue was extracted into toluene $(2 \mathrm{~mL})$ and filtered through celite. The solution was concentrated to $1 \mathrm{~mL}$ under reduced pressure before being cooled to $-30{ }^{\circ} \mathrm{C}$ to give the compound as orange crystals.

${ }^{1} \mathrm{H}$ NMR $\left(\mathrm{C}_{6} \mathrm{D}_{6}, 600 \mathrm{MHz}\right) \delta 7.39\left(\mathrm{~d}, J_{\mathrm{HH}}=7.9 \mathrm{~Hz}, 8 \mathrm{H}, \mathrm{ArH}\right) ; 7.20\left(\mathrm{t}, J_{\mathrm{HH}}=7.9 \mathrm{~Hz}, 8 \mathrm{H}, \mathrm{ArH}\right) ; 7.08\left(\mathrm{t}, J_{\mathrm{HH}}=7.2\right.$ $\mathrm{Hz}, 4 \mathrm{H}, \mathrm{ArH}) ; 7.05(\mathrm{~s}, 2 \mathrm{H}, \mathrm{ArH}) ; 6.43\left(\mathrm{~s}, 2 \mathrm{H},\left(\mathrm{CHPh}_{2}\right)_{2}\right) 5.74(\mathrm{~s}, 10 \mathrm{H}), \mathrm{C}_{5} \mathrm{H}_{5} ; 3.44(\mathrm{~m}, 4 \mathrm{H}, \mathrm{THF}) ; 1.17$ (s, 9H, $\left.\mathrm{C}\left(\mathrm{CH}_{3}\right)_{3}\right) ; 1.10-1.07(\mathrm{~m}, 4 \mathrm{H}, \mathrm{THF})$.

${ }^{13} \mathrm{C}$ NMR (C $\left.{ }_{6} \mathrm{D}_{6}, 125 \mathrm{MHz}\right) \delta 165.7$ (ArC); $152.8(\operatorname{ArC}) ; 144.7$ (ArC); 137.9 (ArC); $130.2(\operatorname{ArC}) ; 129.3(\operatorname{ArC}) ;$

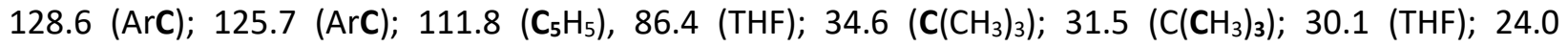
$\left(\left(\mathrm{CH}\left(\mathrm{Ph}_{2}\right)\right)_{2}\right)$.

\subsubsection{8 $\quad\left[\mathrm{Cp}_{2} \mathrm{Zr}(\mathrm{Bn})(\mathrm{NHDMP})\right] \quad 19 \quad \mathrm{C}_{25} \mathrm{H}_{27} \mathrm{NZr}$}

To a stirred solution of $\left[\mathrm{Bn}_{2} \mathrm{ZrCp}_{2}\right](100 \mathrm{mg}, 0.03 \mathrm{mmol})$ in $\mathrm{C}_{6} \mathrm{D}_{6}(0.30 \mathrm{~mL})$ was added [DMPNH $\mathrm{H}_{2}$ ( $30 \mathrm{mg}$, $0.03 \mathrm{mmol}$ ) and the reaction was transferred to a NMR tube fitted with a Young's tap. The reaction was heated at $80{ }^{\circ} \mathrm{C}$ for 2 days until there was no remaining evidence of the free amine by ${ }^{1} \mathrm{H}$ NMR spectroscopy. The NMR tube was taken into a nitrogen filled glovebox where it was diluted with toluene $(1 \mathrm{~mL})$ and the solvent was removed under reduced pressure. The residue was extracted into minimal hexane $(4 \mathrm{~mL})$ and cooled to $-30^{\circ} \mathrm{C}$ to give the product as pale yellow crystals.

${ }^{1} \mathrm{H}$ NMR $\left(\mathrm{C}_{6} \mathrm{D}_{6}, 600 \mathrm{MHz}\right) \delta 7.34\left(\mathrm{t}, J_{\mathrm{HH}}=6.9 \mathrm{~Hz}, 2 \mathrm{H}, \mathrm{ArH}\right) ; 7.22\left(\mathrm{~d}, J_{\mathrm{HH}}=7.7 \mathrm{~Hz}, 2 \mathrm{H}, \operatorname{ArH}\right) ; 7.11(\mathrm{~m}, 2 \mathrm{H}, \operatorname{ArH})$; $7.05\left(\mathrm{t}, J_{\mathrm{HH}}=7.2 \mathrm{~Hz}, 1 \mathrm{H}, \mathrm{ArH}\right) ; 6.95\left(\mathrm{t}, \mathrm{J}_{\mathrm{HH}}=8.1 \mathrm{~Hz}, 1 \mathrm{H}, \mathrm{ArH}\right) ; 5.44\left(\mathrm{~s}, 10 \mathrm{H}, \mathrm{C}_{5} \mathrm{H}_{5}\right) ; 3.50(\mathrm{br} \mathrm{s}, 1 \mathrm{H}, \mathrm{NH}) ; 2.25$ $\left(\mathrm{s}, 2 \mathrm{H}, \mathrm{CH}_{2} \mathrm{Ph}\right) ; 2.14\left(\mathrm{~s}, 6 \mathrm{H}, 2,6-\mathrm{MeC}_{6} \mathrm{H}_{3}\right)$.

${ }^{13} \mathrm{C}$ NMR $\left(\mathrm{C}_{6} \mathrm{D}_{6}, 125 \mathrm{MHz}\right) \delta 155.7(\operatorname{ArC}) ; 154.8(\operatorname{ArC}) ; 137.9(\operatorname{ArC}) ; 131.3(\operatorname{ArC}) ; 129.3(\operatorname{ArC}) ; 125.7(\operatorname{ArC}) ;$ 122.8 (ArC); 120.9 (ArC); $110.8\left(\mathrm{C}_{5} \mathrm{H}_{5}\right) ; 47.0\left(\mathrm{CH}_{2} \mathrm{Ph}\right) ; 20.9\left(2,6-\mathrm{MeC}_{6} \mathrm{H}_{3}\right)$. 


\subsubsection{9 $[\mathrm{Ar} * \mathrm{Cl}] \quad 26 \quad \mathrm{C}_{36} \mathrm{H}_{33} \mathrm{Cl}$}

In a $250 \mathrm{~mL}$ RB flask with stirring, [Ar* $\left.\mathrm{NH}_{2}\right](2.68 \mathrm{~g}, 5.57 \mathrm{mmol})$ was dissolved in 1:2 $\mathrm{H}_{2} \mathrm{O}(50 \mathrm{~mL})$ to THF $(100 \mathrm{~mL})$ mixture. Concentrated $\mathrm{HCl}$ was added $(10 \mathrm{~mL}, 12.1 \mathrm{M})$ and the temperature of the reaction was brought down to $0^{\circ} \mathrm{C}$ using an ice bath. The solution was treated with 4 equivalents of sodium nitrite (1.50 g, $22.3 \mathrm{mmol})$ in $\mathrm{H}_{2} \mathrm{O}(10 \mathrm{~mL})$ added dropwise over 5 minutes before being left to stir for four hours, after which time the solution had turned yellow. A solution of $\left[\mathrm{NaN}_{3}\right](1.44 \mathrm{~g}, 0.02 \mathrm{~mol})$ in $\mathrm{H}_{2} \mathrm{O}(10 \mathrm{~mL})$ was added dropwise over 5 minutes while maintaining an internal reaction temperature of under $5{ }^{\circ} \mathrm{C}$. The reaction was left to stir for four hours. The target compound was extracted into toluene $(3 \times 50 \mathrm{~mL})$ and washed with sodium bicarbonate $(2 \times 100 \mathrm{~mL}), \mathrm{H}_{2} \mathrm{O}(2 \times 50 \mathrm{~mL})$ and brine $(2 \times 50 \mathrm{~mL})$ before the toluene fraction was dried over $\left[\mathrm{MgSO}_{4}\right]$ and filtered through celite. The volatiles were removed to give an offwhite powder. The compound was purified by fractional recrystalisation from minimal toluene. Yield 1.19 g (14\%).

${ }^{1} \mathrm{H} \mathrm{NMR}\left(\mathrm{C}_{6} \mathrm{D}_{6}, 300 \mathrm{MHz}\right) \delta 7.14-6.98(\mathrm{~m}, 22 \mathrm{H}, \mathrm{ArH}) ; 6.19\left(\mathrm{~s}, 2 \mathrm{H}\left(\mathrm{CHPh}_{2}\right)_{2}\right) ; 1.02\left(\mathrm{~s}, 9 \mathrm{H}, \mathrm{C}\left(\mathrm{CH}_{3}\right)_{3}\right)$.

${ }^{13} \mathrm{C}$ NMR (C $\left.\mathrm{C}_{6} \mathrm{D}_{6} 125 \mathrm{MHz}\right) \delta 153.9$ (ArC); 144.9 (ArC); $143.9(\operatorname{ArC}) ; 139.5(\operatorname{ArC}) ; 138.5(\operatorname{ArC}) ; 129.0(\operatorname{ArC}) ;$

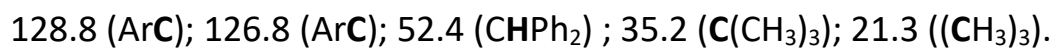

\section{$5.2{ }^{1} \mathrm{H}$ And ${ }^{13} \mathrm{C}$ NMR Spectra for Novel Compounds}

The following compounds were all run in deuterated benzene at $25^{\circ} \mathrm{C}$. Impurities in the spectra are noted on each individual sample. All spectra were internally referenced to the residual solvent signal. 
5.2.1 ${ }^{1} \mathrm{H}$ NMR and ${ }^{13} \mathrm{C}$ Spectra of 2 (THF impurity)

utu
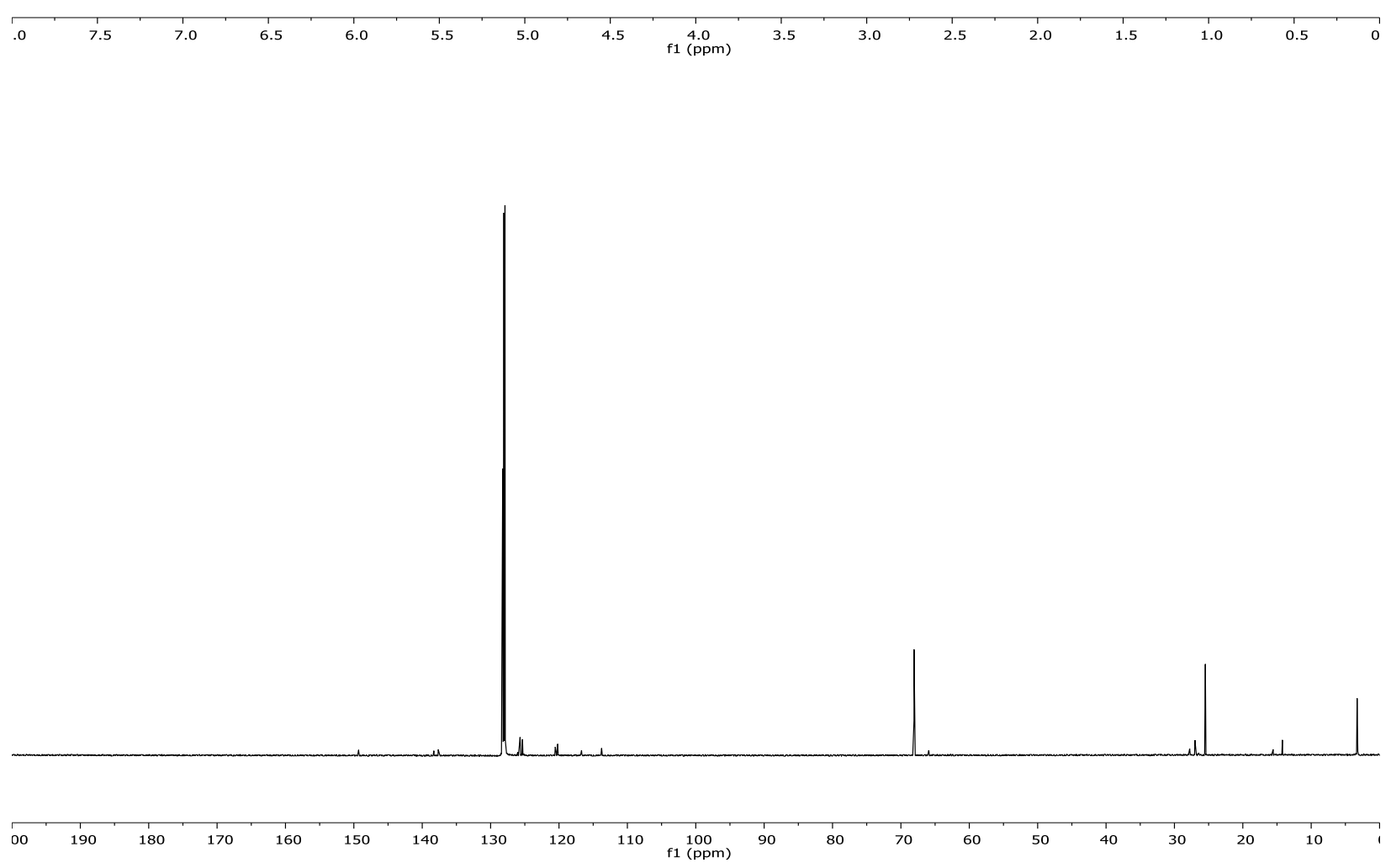

133 


\subsection{2 ${ }^{1} \mathrm{H}$ NMR and ${ }^{13} \mathrm{C}$ Spectra of 3}
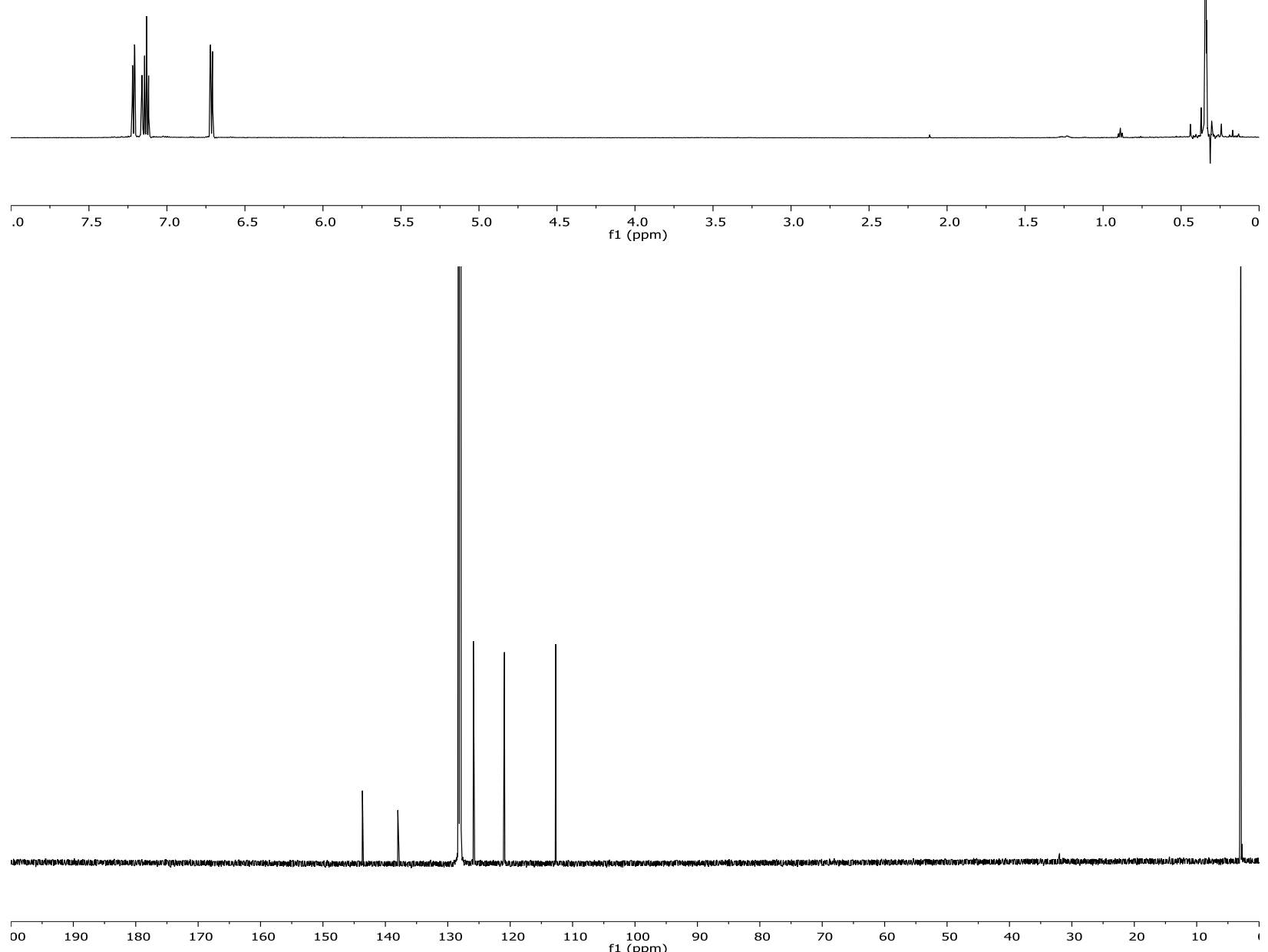


\subsection{3 ${ }^{1} \mathrm{H}$ NMR and ${ }^{13} \mathrm{C}$ Spectra of 4}
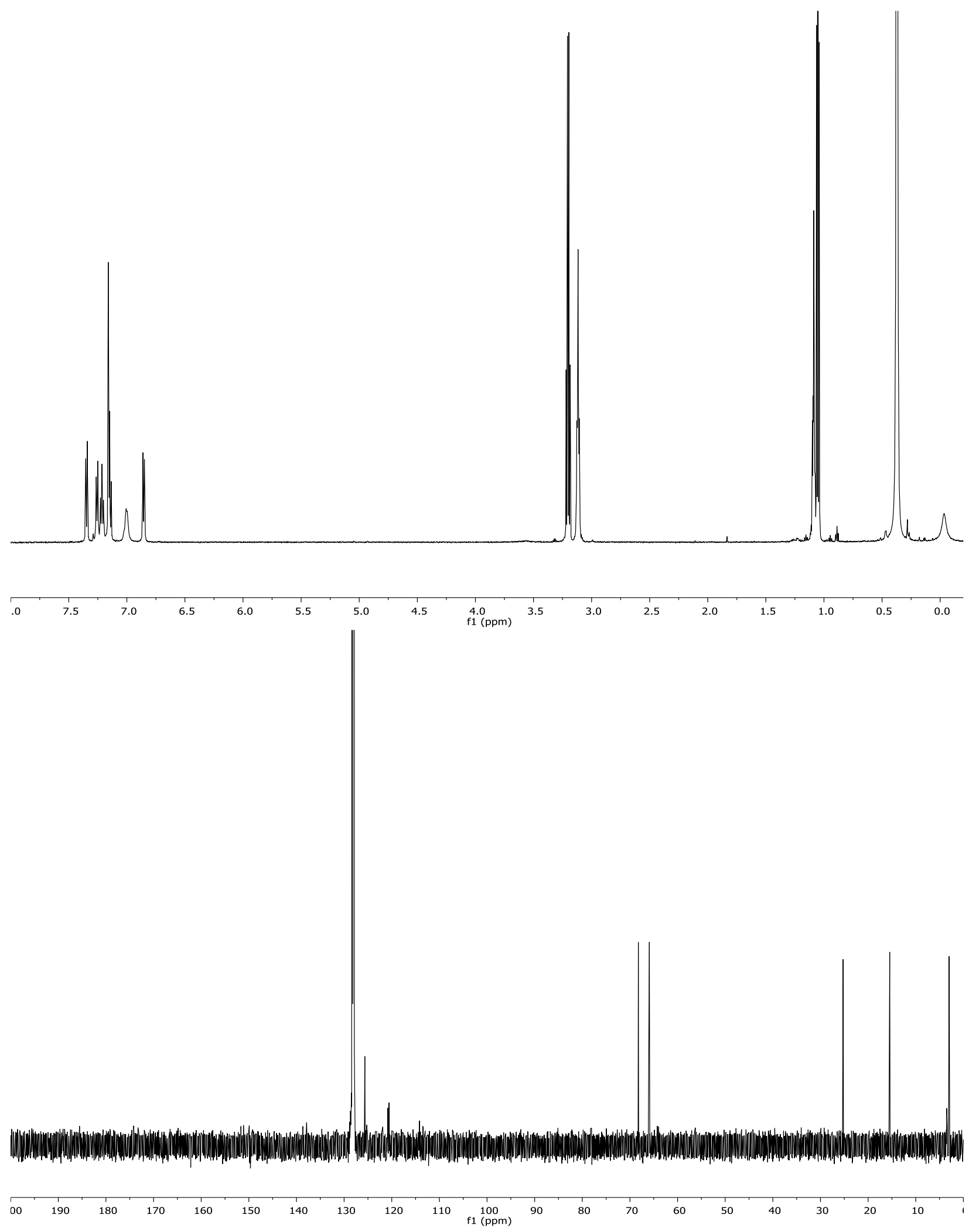
5.2.4 ${ }^{1} \mathrm{H}$ NMR and ${ }^{13} \mathrm{C}$ Spectra of 6
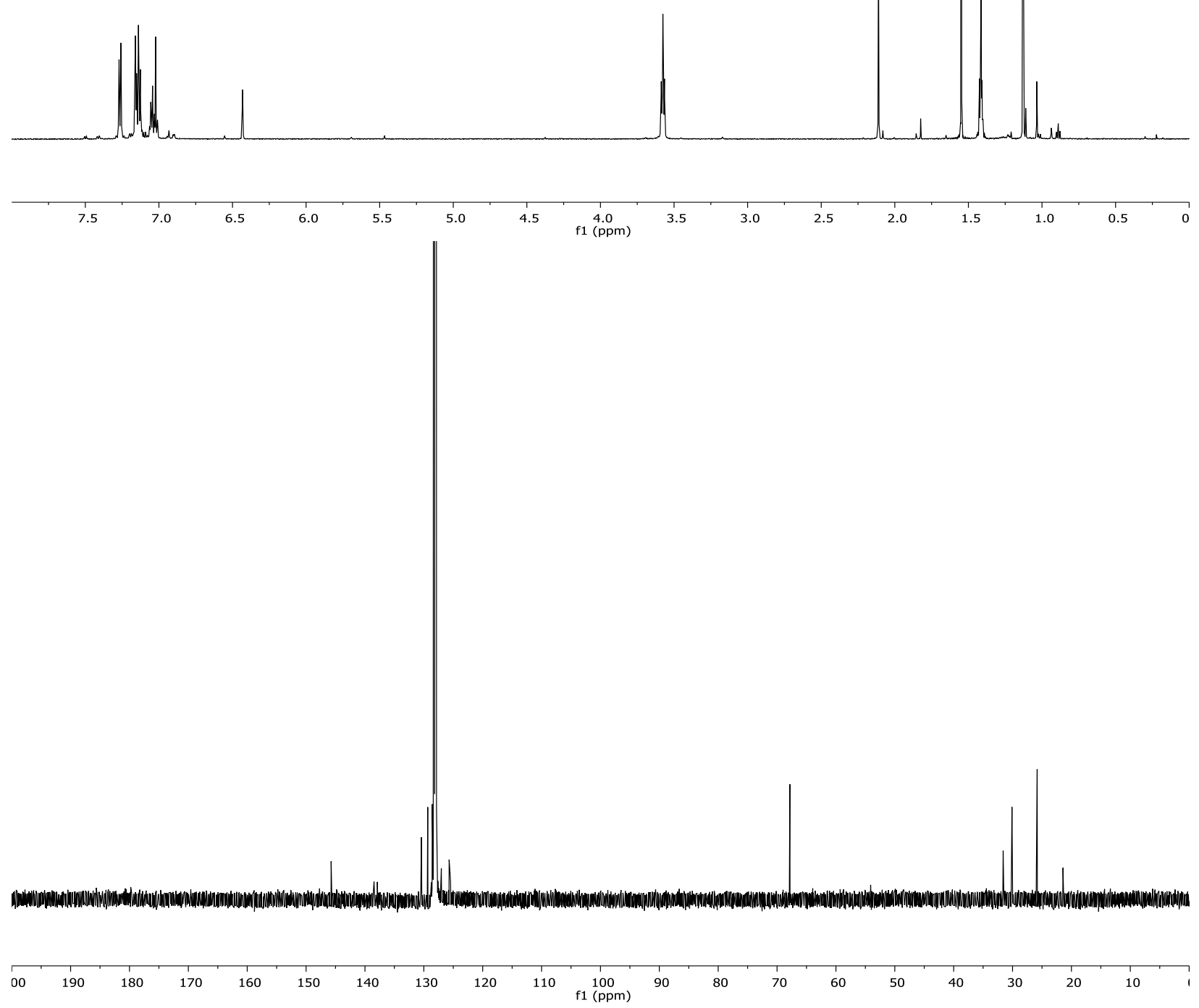

136 


\subsection{5 ${ }^{1} \mathrm{H}$ NMR and ${ }^{13} \mathrm{C}$ Spectra of 7}
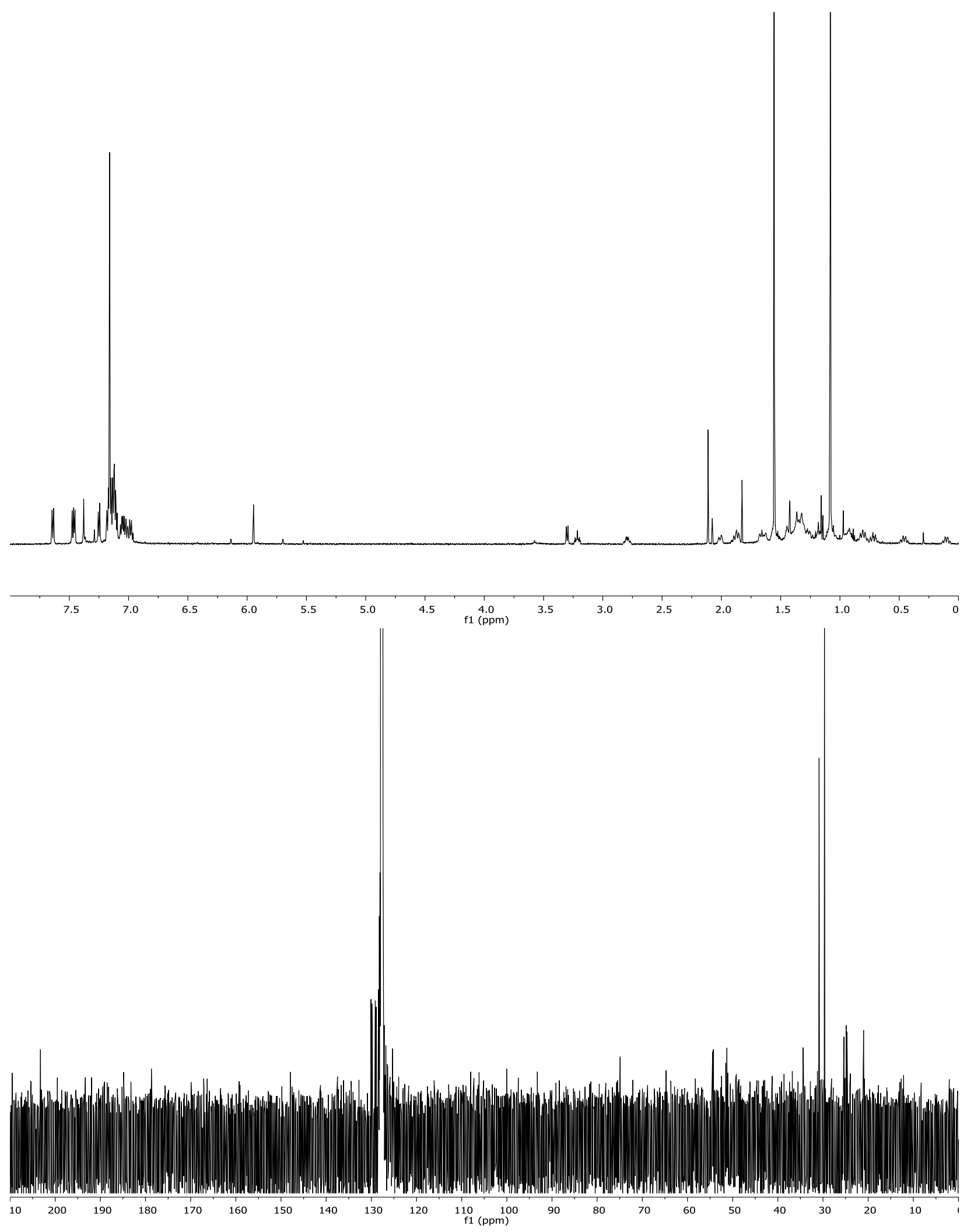


\subsection{6 ${ }^{1} \mathrm{H}$ NMR and ${ }^{13} \mathrm{C}$ Spectra of 8}
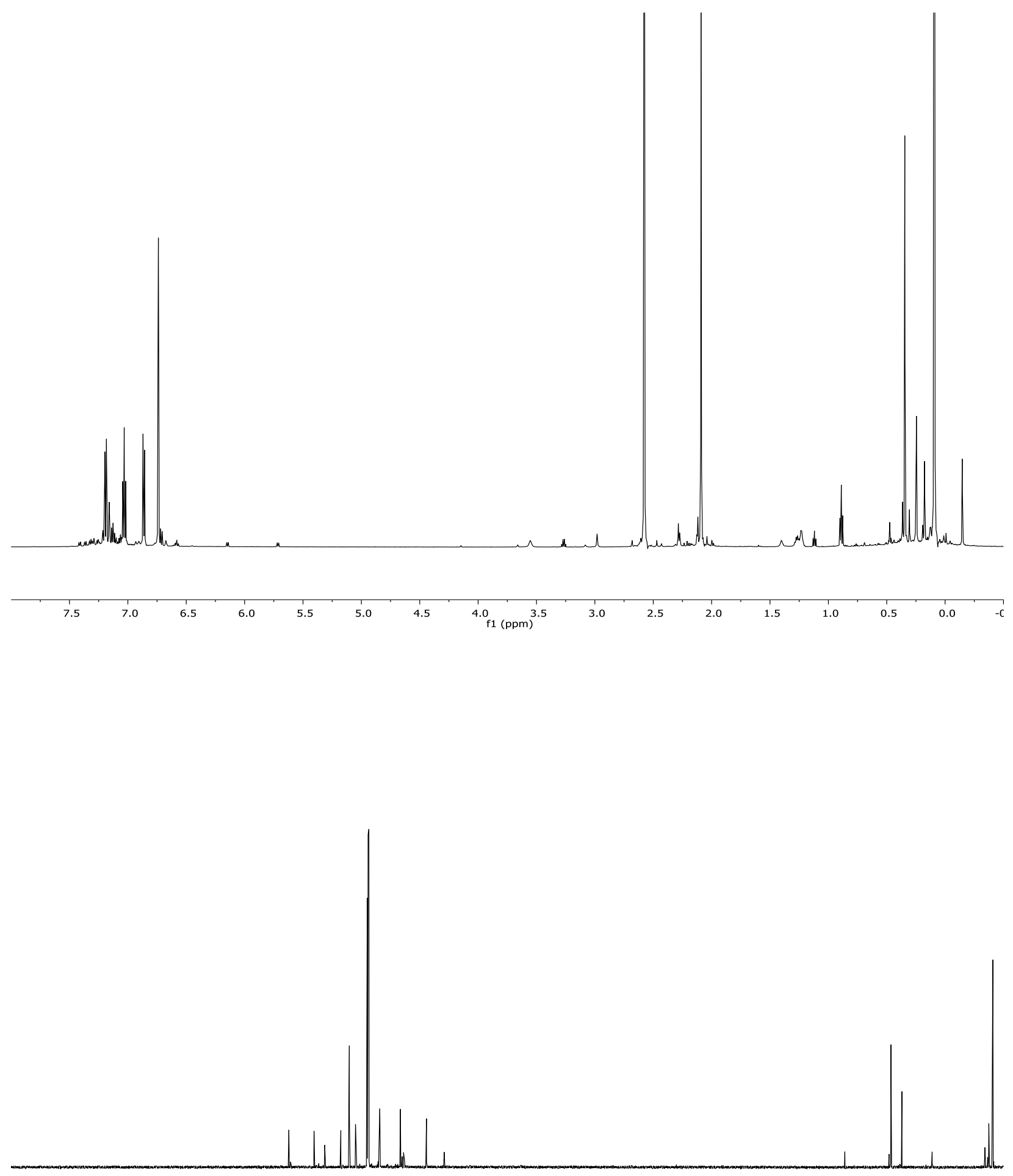

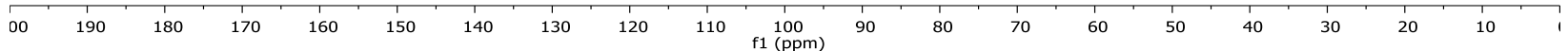




\subsection{7 ${ }^{1} \mathrm{H}$ NMR and ${ }^{13} \mathrm{C}$ Spectra of 9}
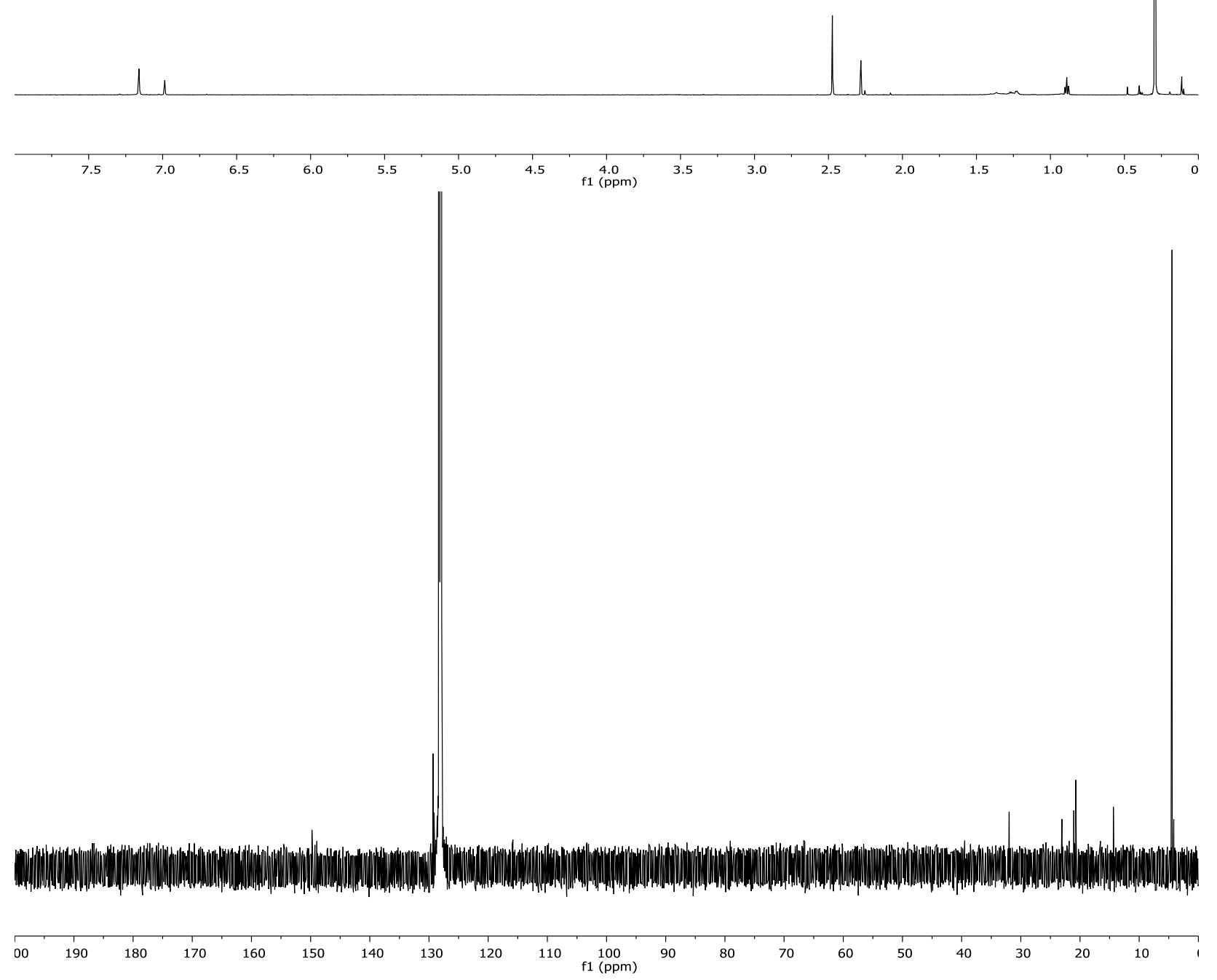


\subsection{8 ${ }^{1} \mathrm{H}$ NMR and ${ }^{13} \mathrm{C}$ Spectra of 10}
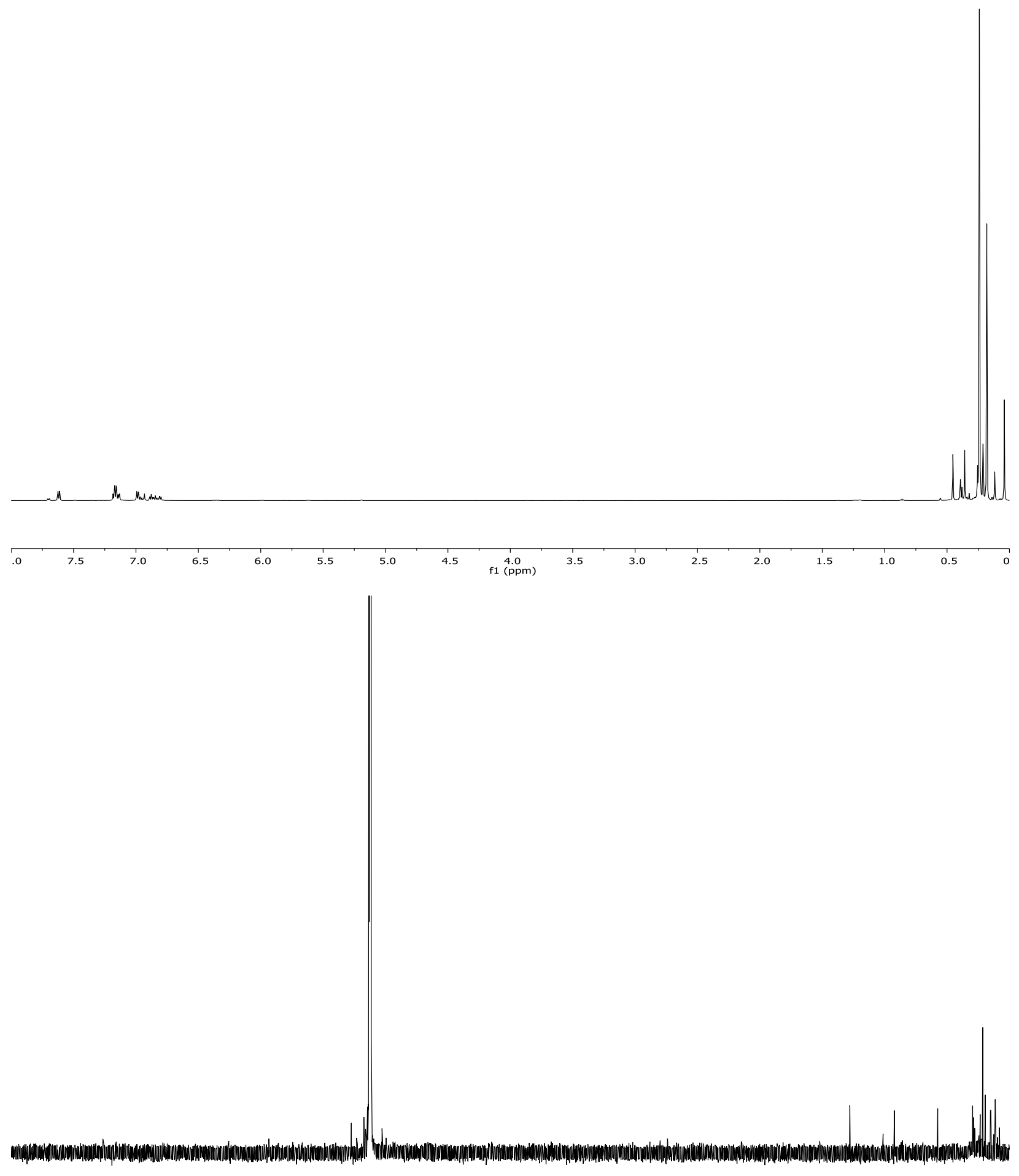

$\begin{array}{lllll}00 & 190 & 180 & 170 & 160\end{array}$

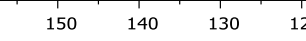

$120 \quad 110 \begin{gathered}100 \\ f 1(\mathrm{ppm})\end{gathered}$ 


\subsection{9 ${ }^{1} \mathrm{H}$ NMR and ${ }^{13} \mathrm{C}$ Spectra of 11 (Toluene impurity)}
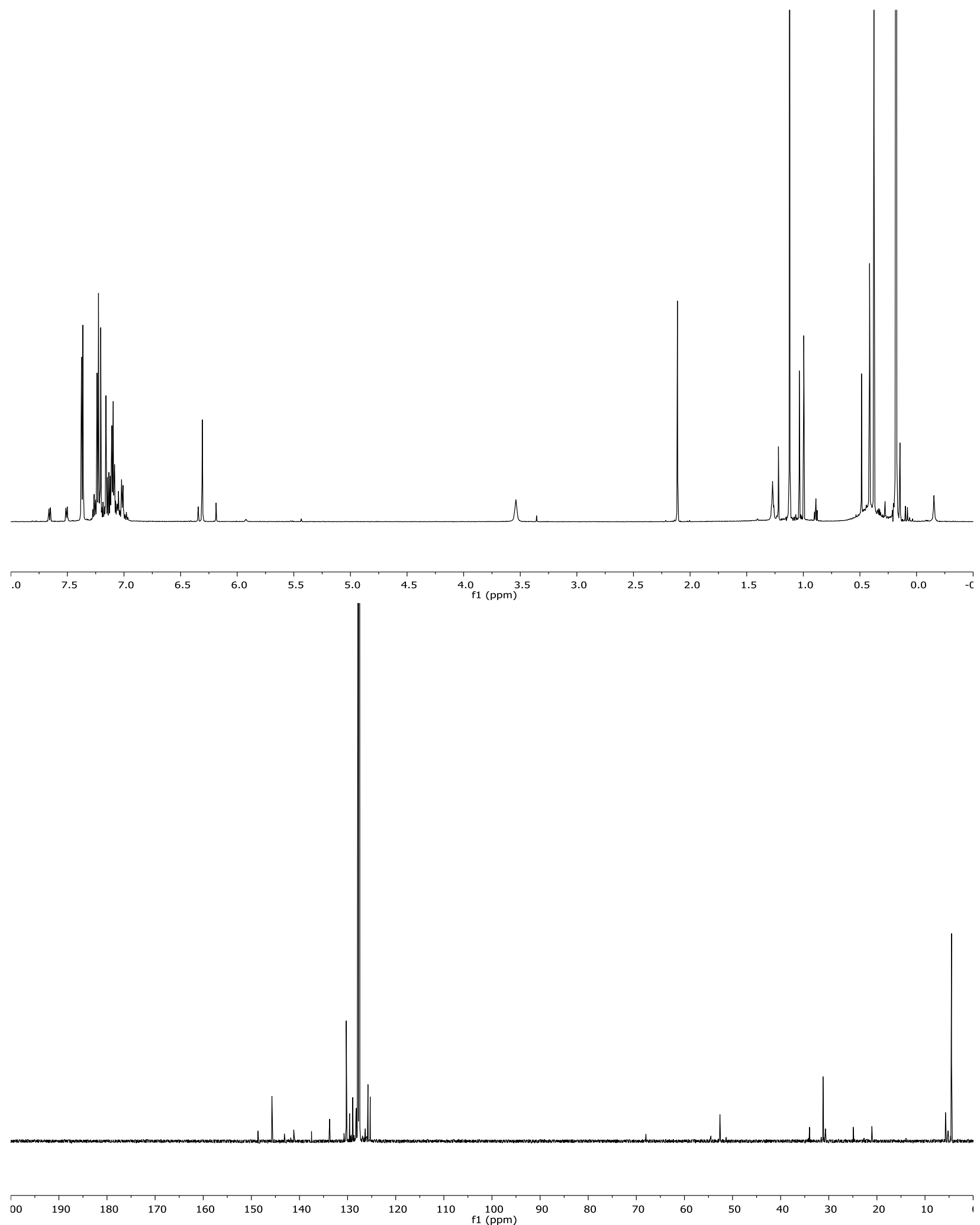


\subsubsection{0 ${ }^{1} \mathrm{H}$ NMR and ${ }^{13} \mathrm{C}$ Spectra of 12 (Toluene impurity)}
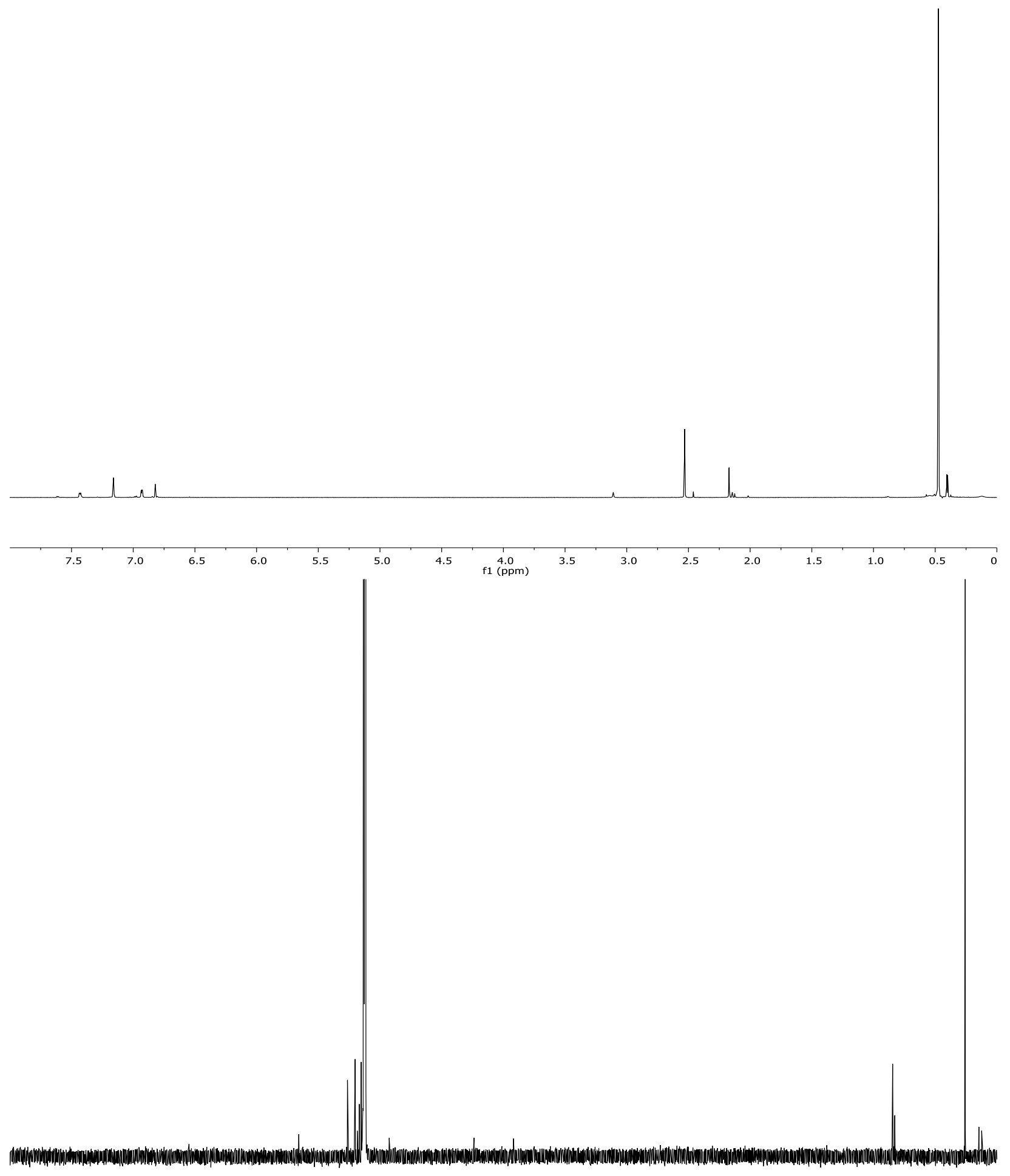


\subsubsection{1 ${ }^{1} \mathrm{H}$ NMR and ${ }^{13} \mathrm{C}$ Spectra of 13}

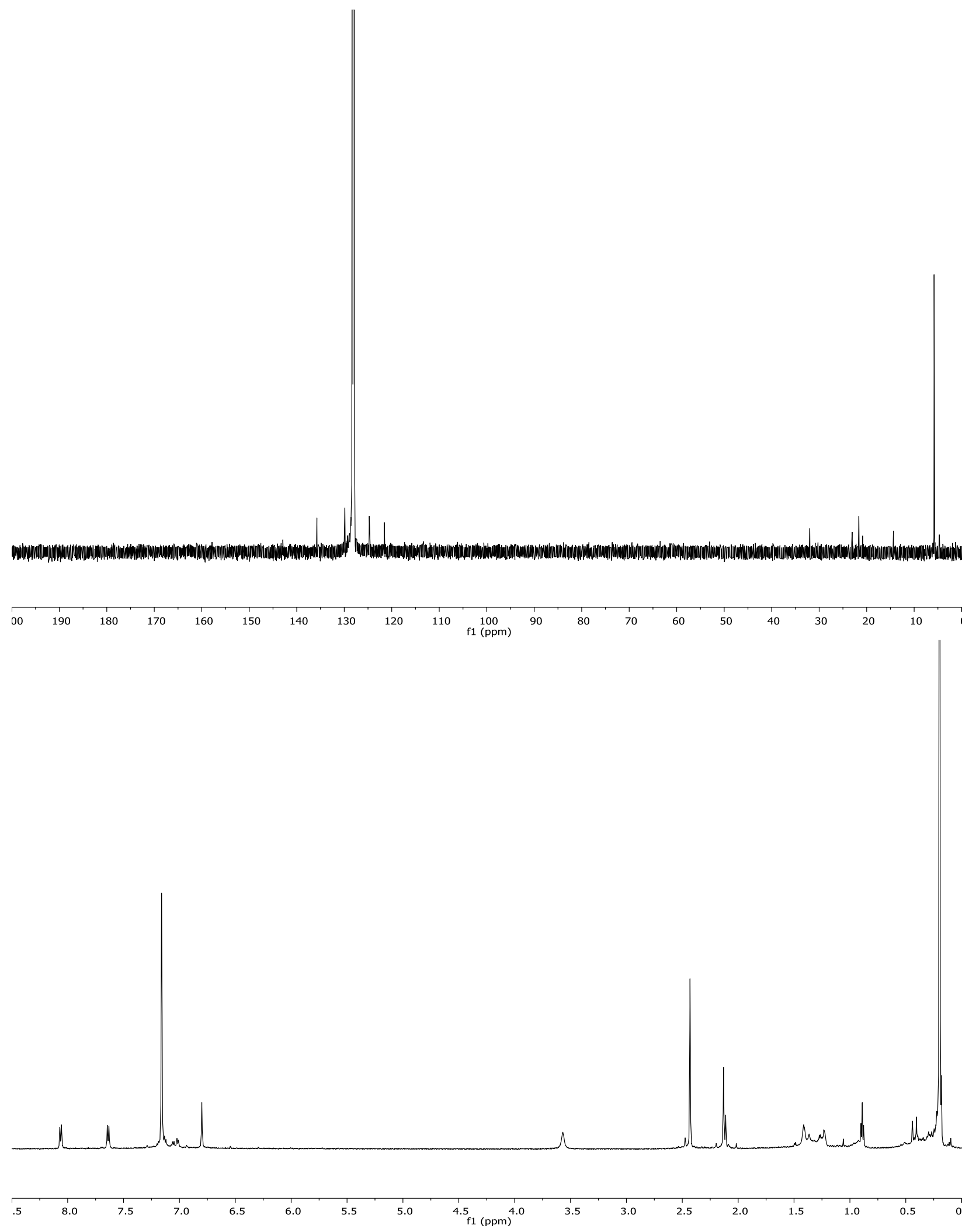




\subsubsection{2 ${ }^{1} \mathrm{H}$ NMR and ${ }^{13} \mathrm{C}$ Spectra of 14}
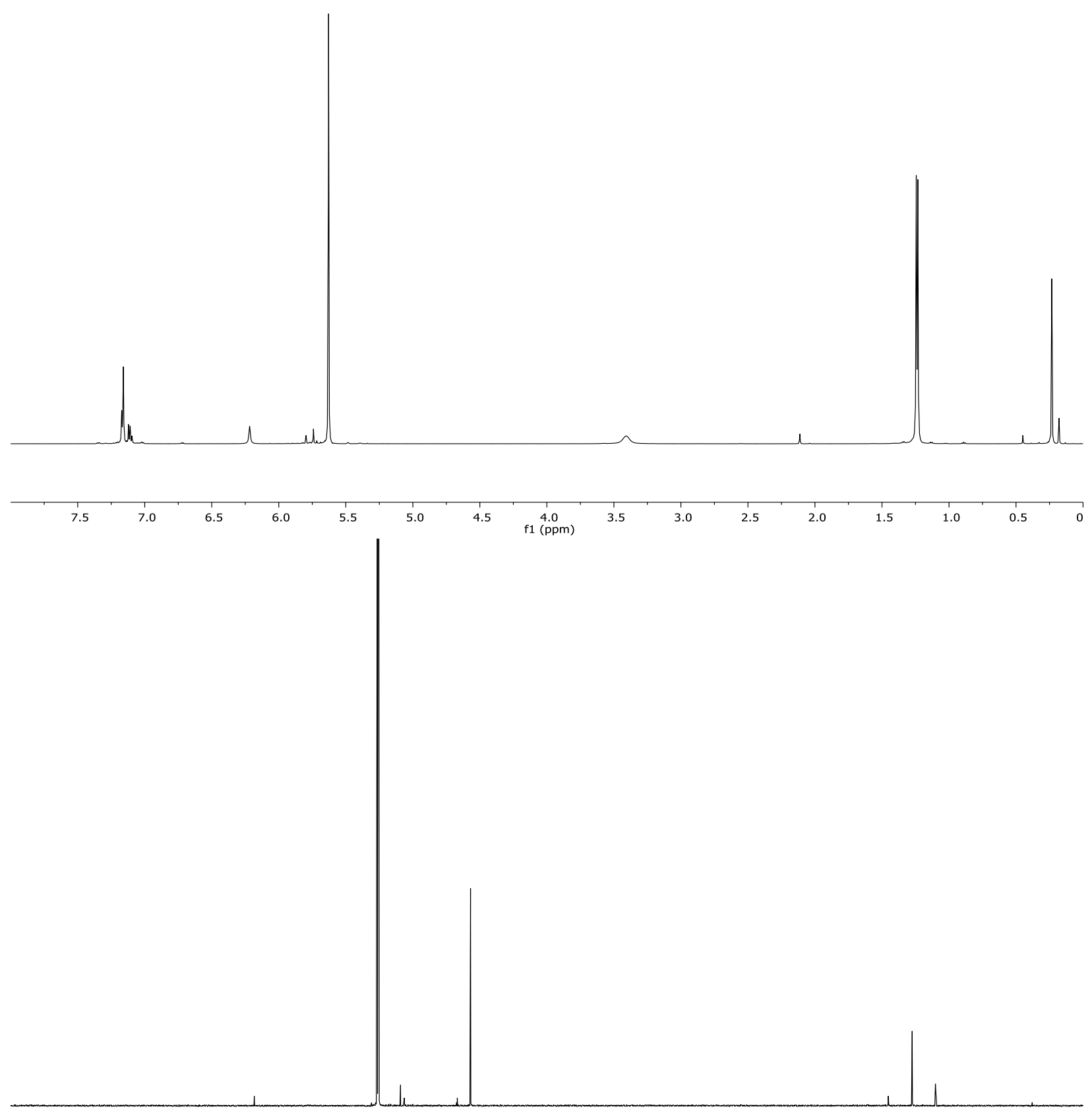

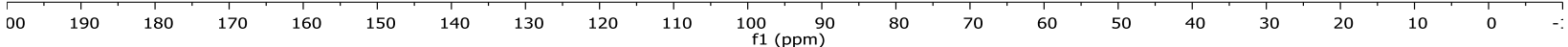




\subsubsection{3 ${ }^{1} \mathrm{H}$ NMR and ${ }^{13} \mathrm{C}$ Spectra of 15}
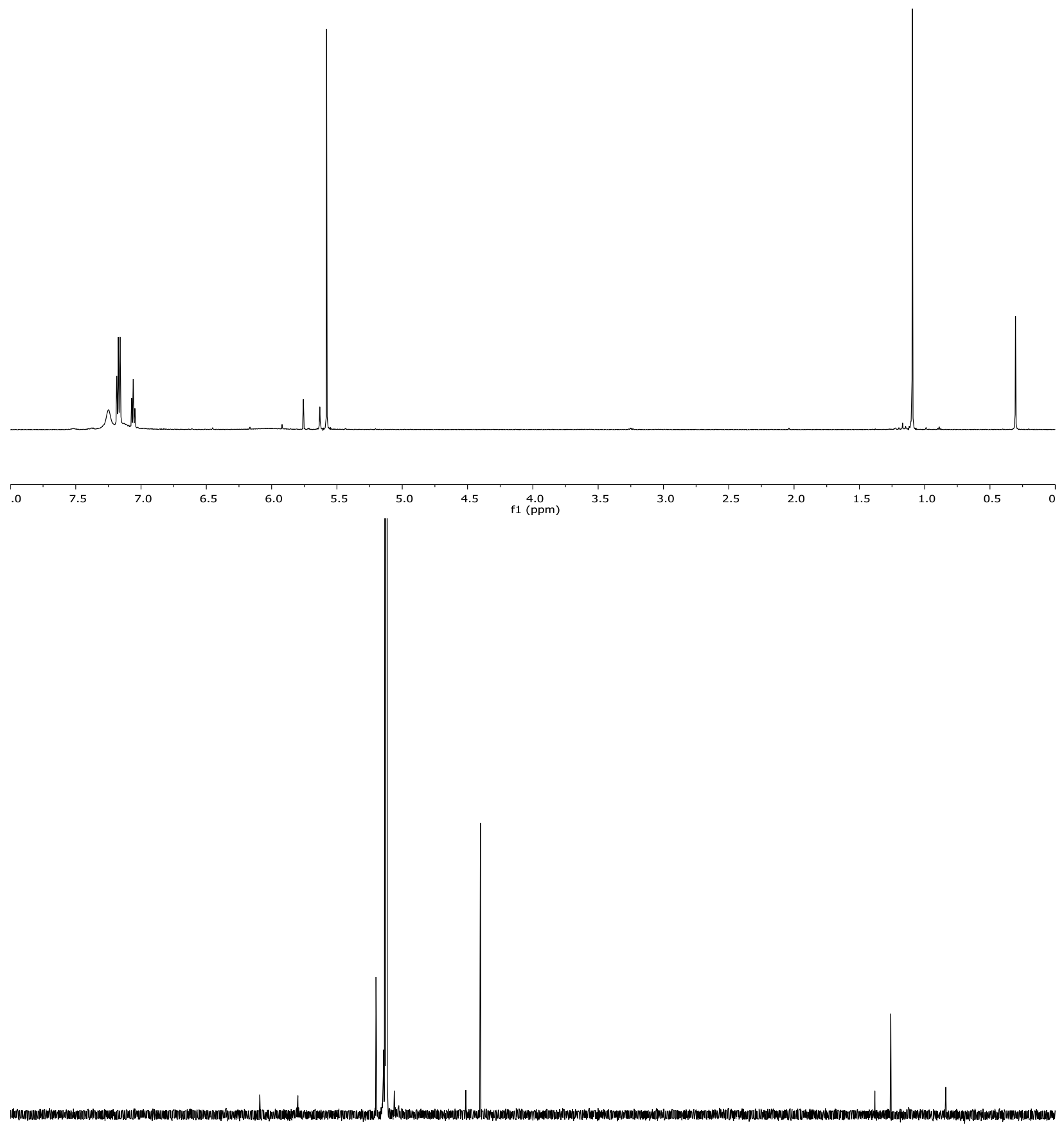

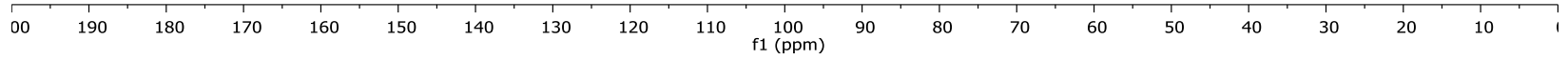


5.2.14 ${ }^{1} \mathrm{H}$ NMR and ${ }^{13} \mathrm{C}$ Spectra of 16
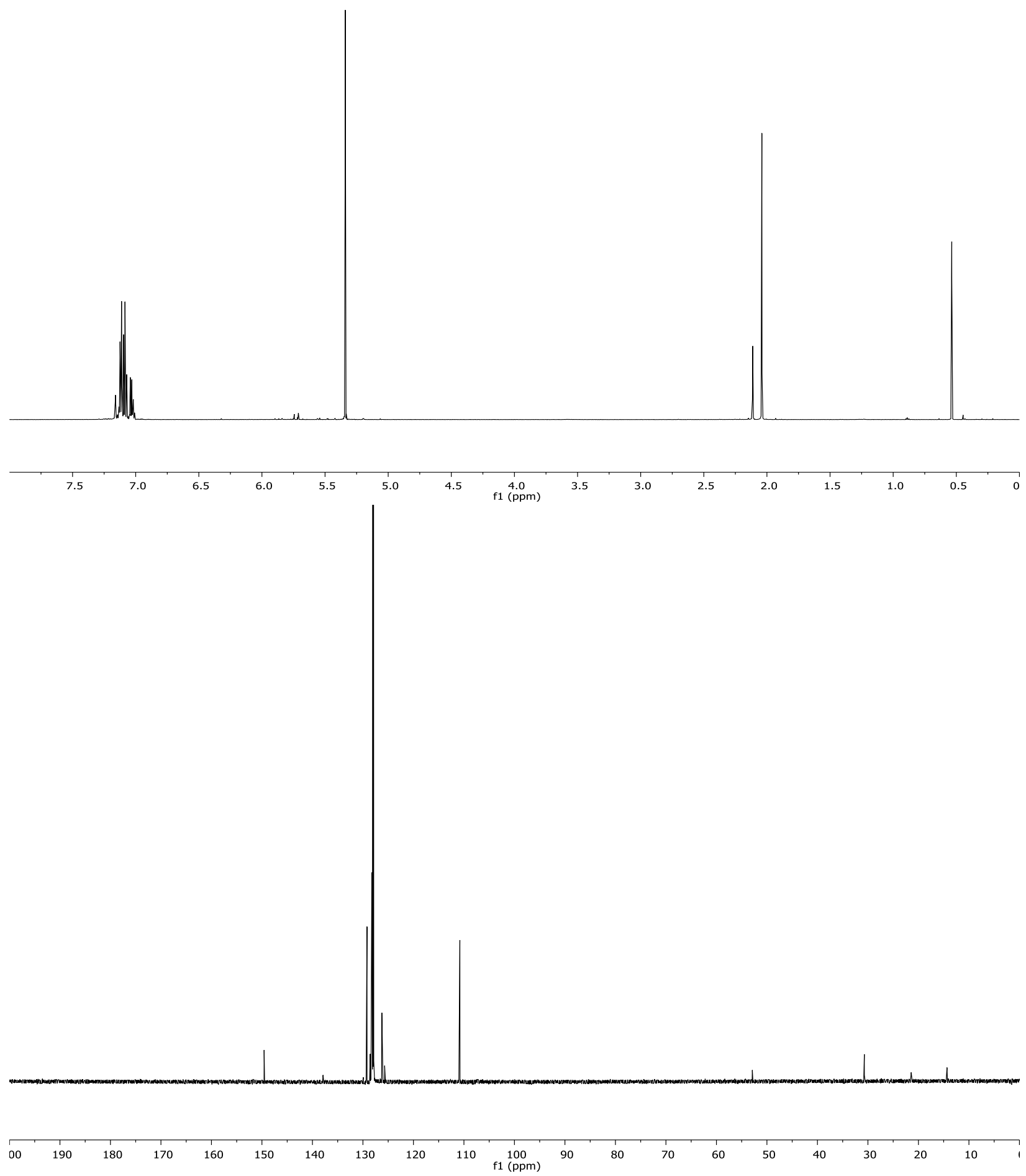

146 


\subsubsection{5 ${ }^{1} \mathrm{H}$ NMR and ${ }^{13} \mathrm{C}$ Spectra of 17}
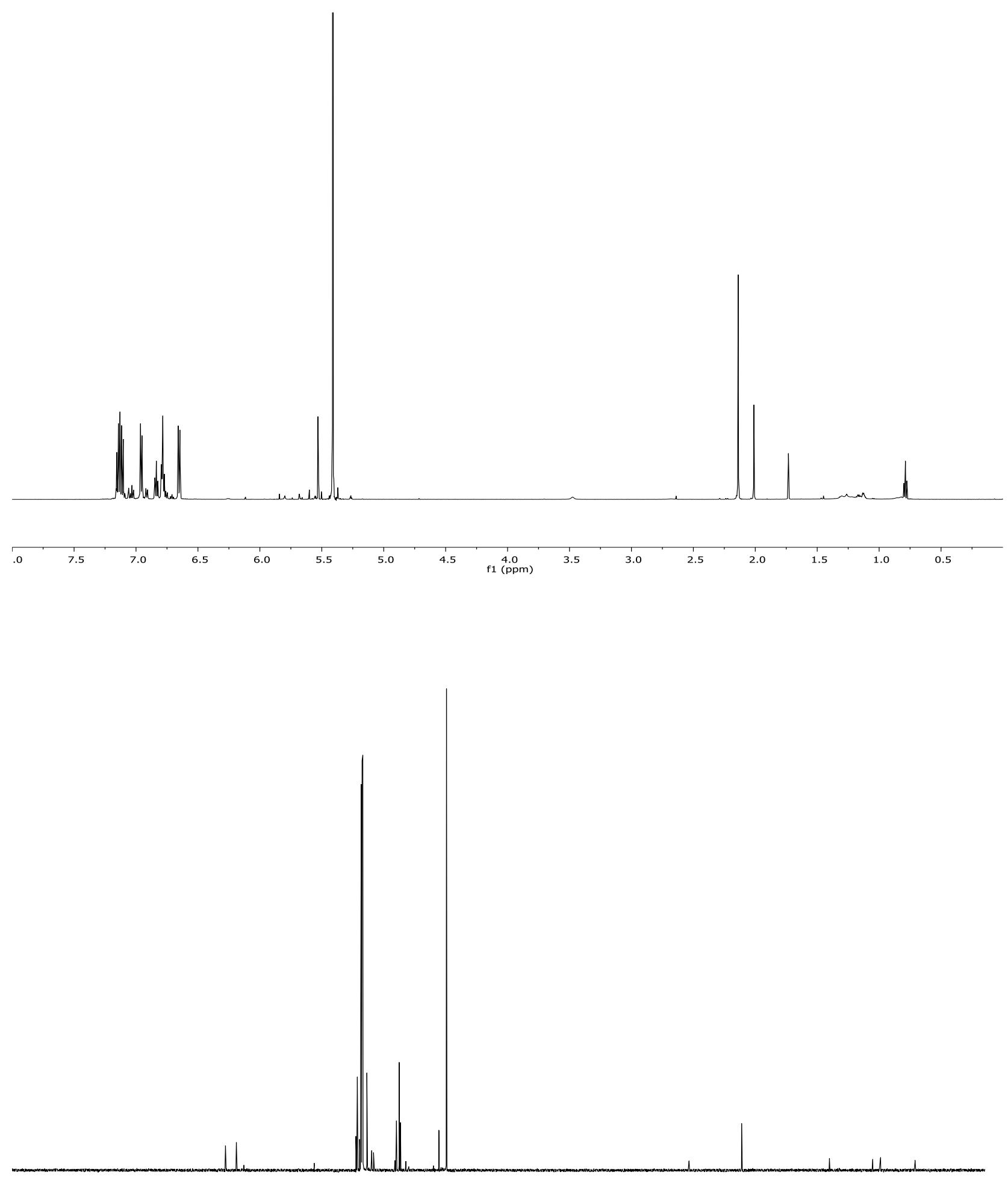


\subsubsection{6 ${ }^{1} \mathrm{H}$ NMR and ${ }^{13} \mathrm{C}$ Spectra of 19 (Toluene impurity)}
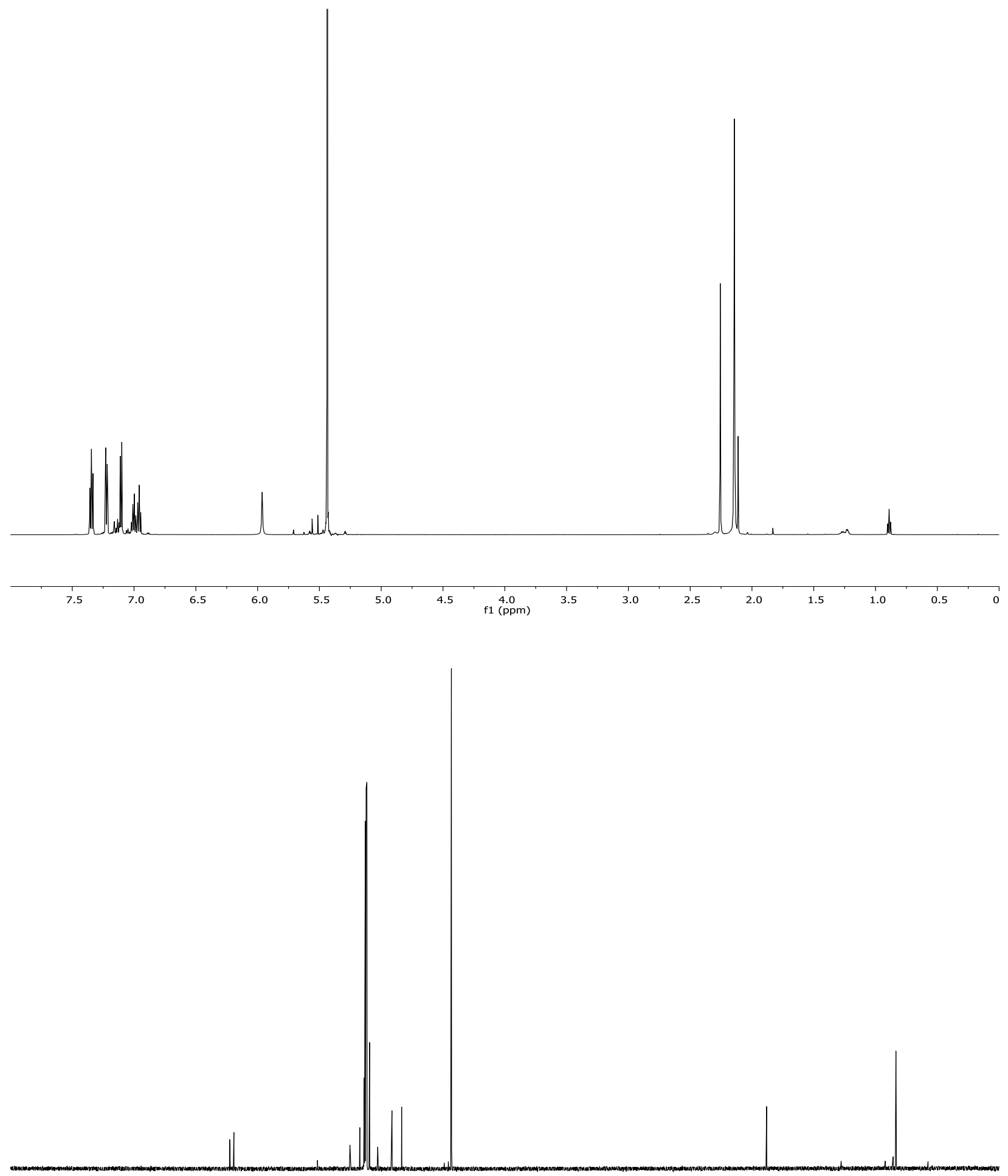

00

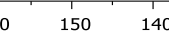

130
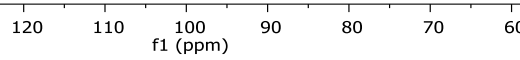

50 


\subsubsection{7 ${ }^{1} \mathrm{H}$ NMR and ${ }^{13} \mathrm{C}$ Spectra of 20 (Toluene impurity)}
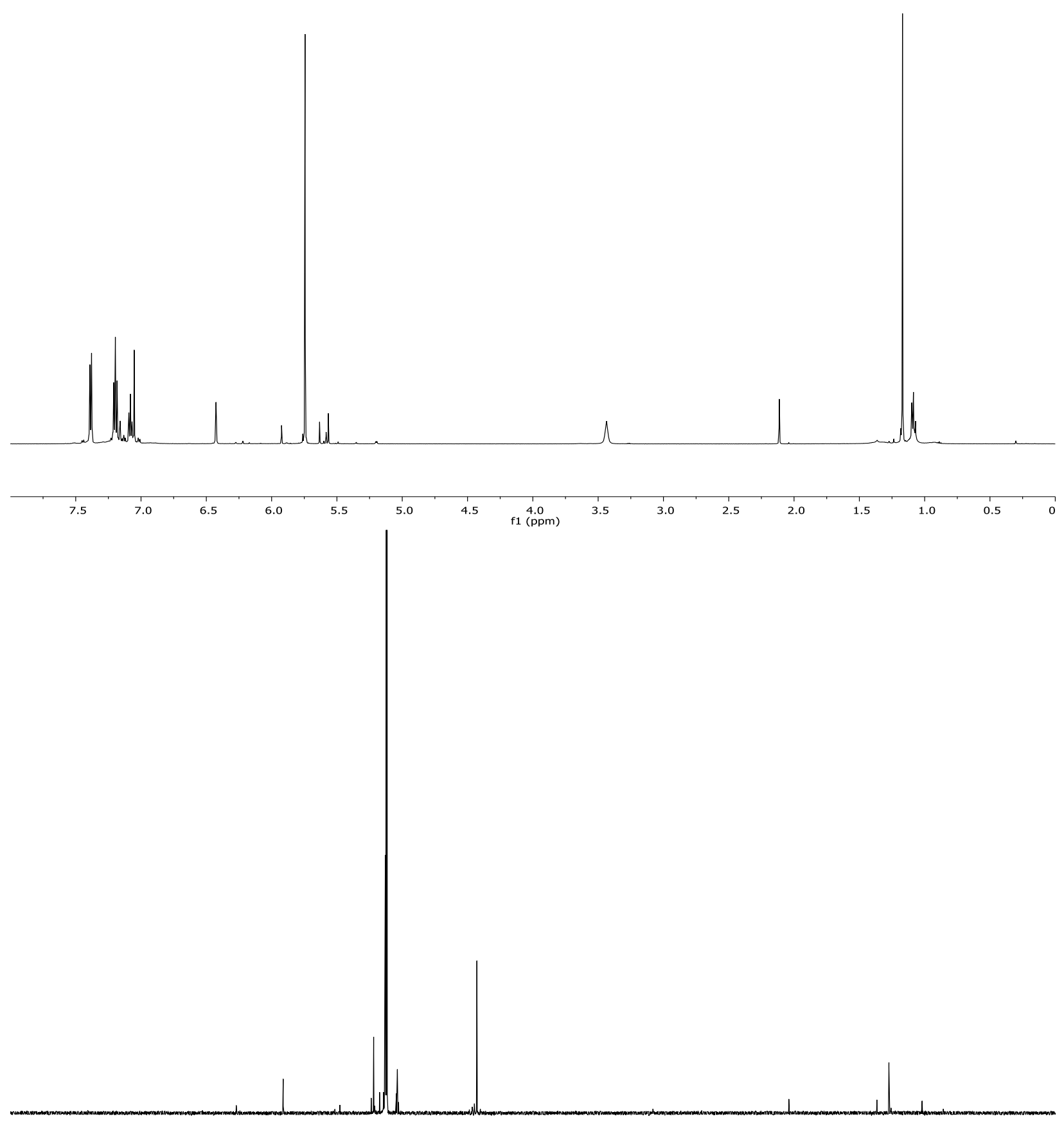


\subsubsection{8 ${ }^{1} \mathrm{H}$ NMR and ${ }^{13} \mathrm{C}$ Spectra of 21}
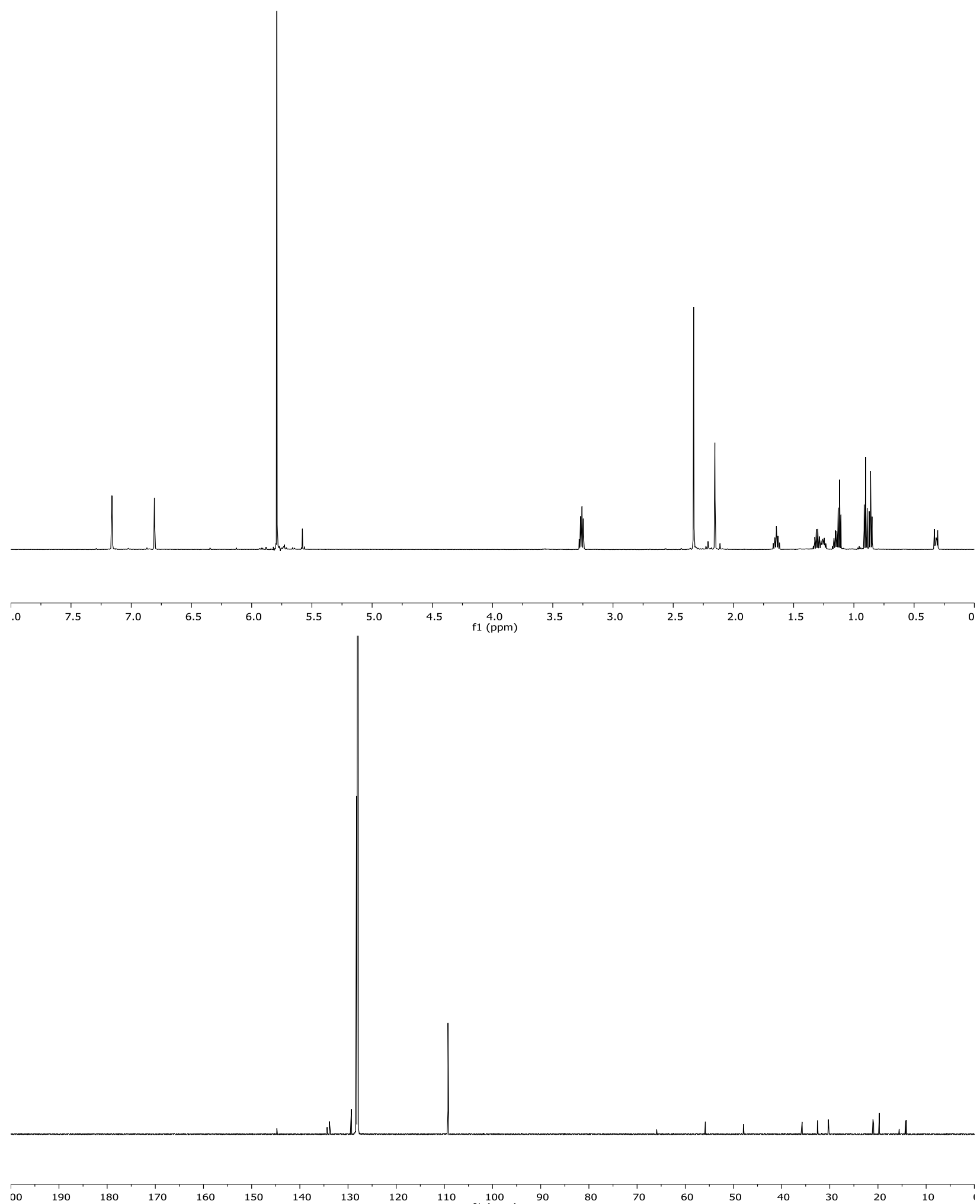

$50 \quad 140$
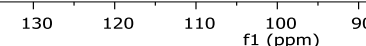
5.2.19 ${ }^{1} \mathrm{H}$ NMR and ${ }^{13} \mathrm{C}$ Spectra of 22 (Toluene impurity)
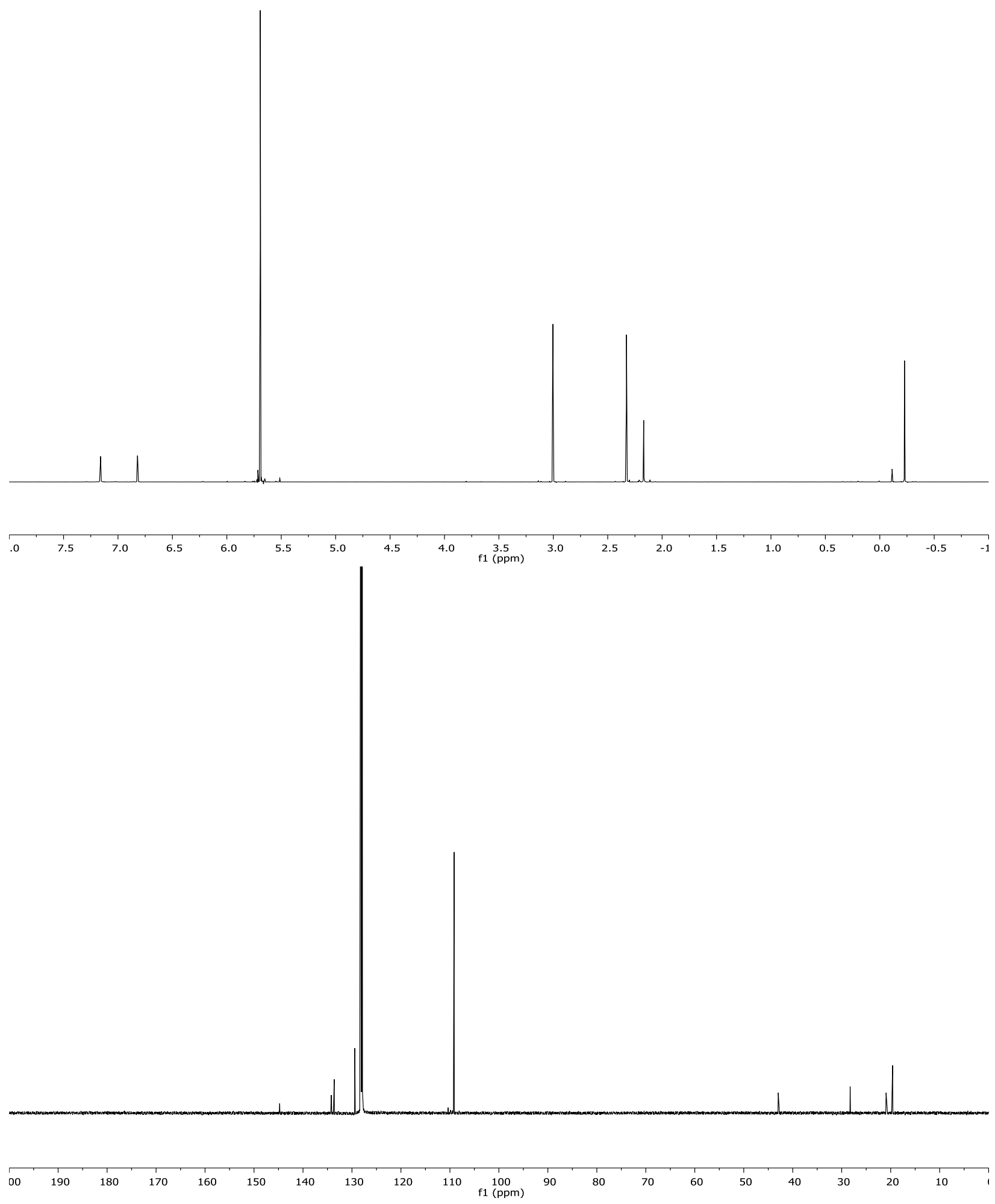

151 


\subsubsection{0 ${ }^{1} \mathrm{H}$ NMR and ${ }^{13} \mathrm{C}$ Spectra of 23}
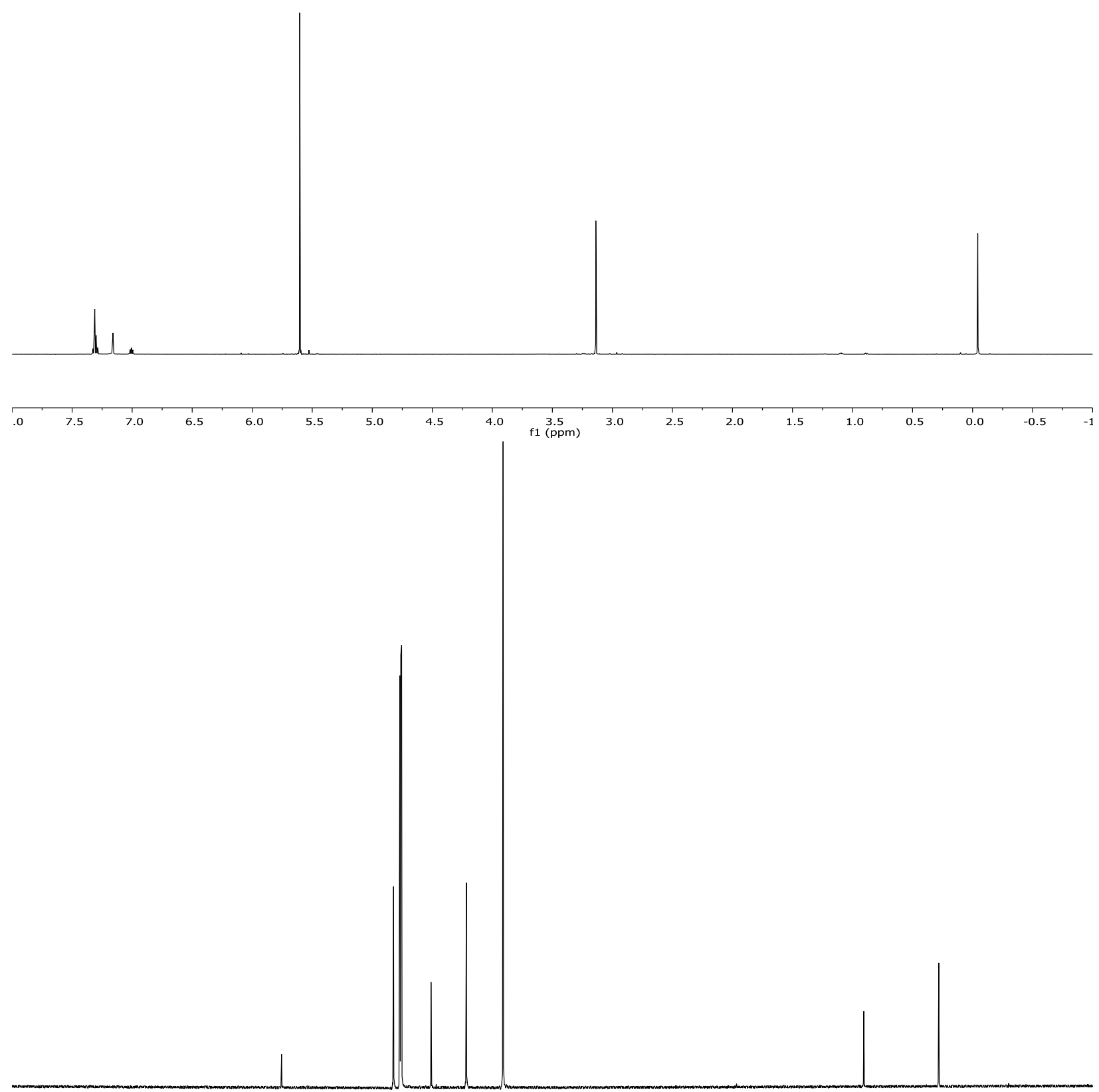

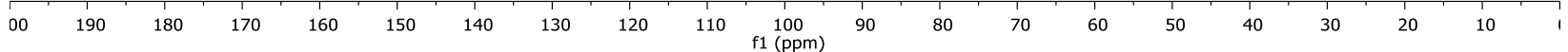


5.2.21 ${ }^{1} \mathrm{H}$ NMR and ${ }^{13} \mathrm{C}$ Spectra of 24
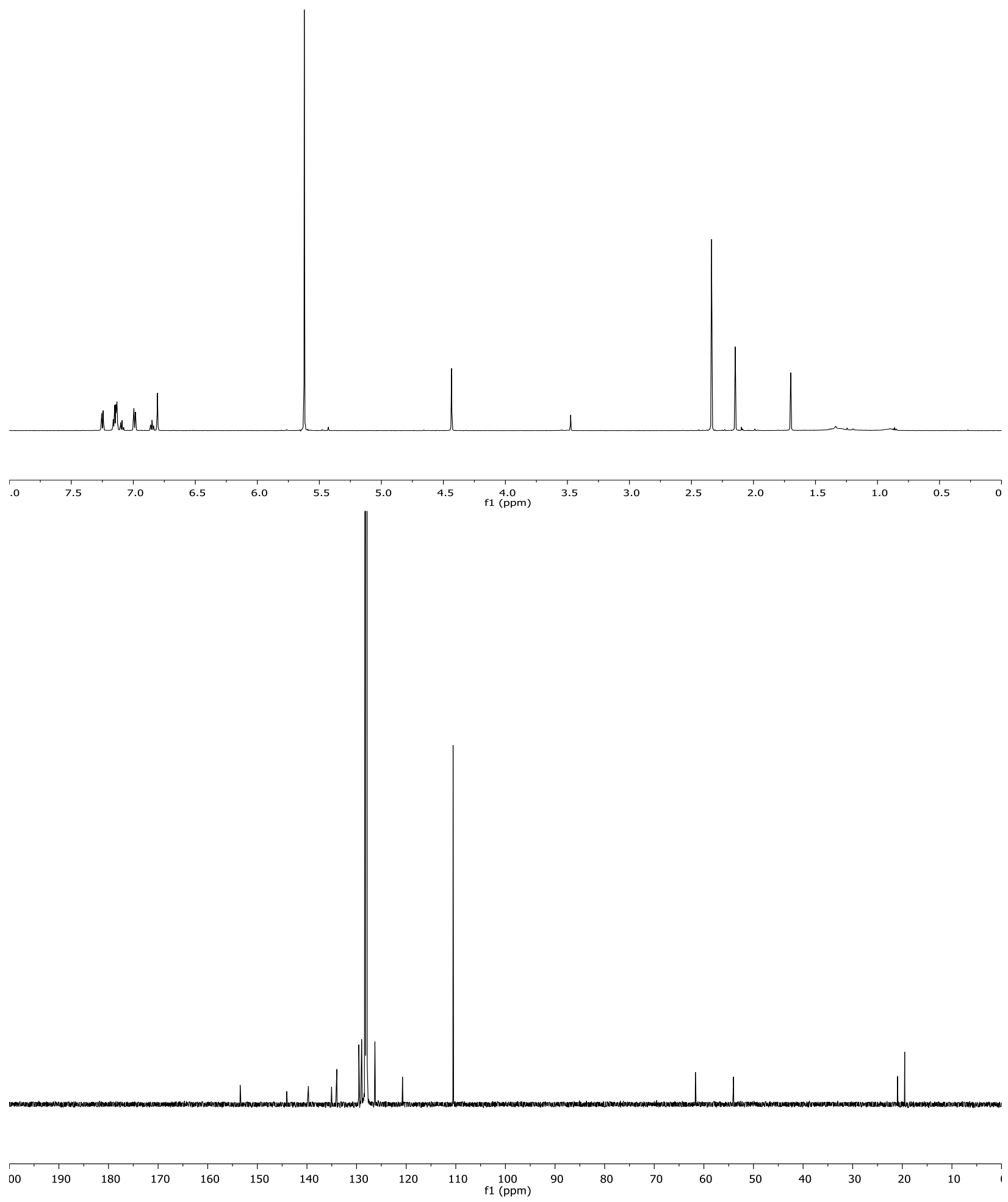

153 


\subsubsection{2 ${ }^{1} \mathrm{H}$ NMR and ${ }^{13} \mathrm{C}$ Spectra of 25}
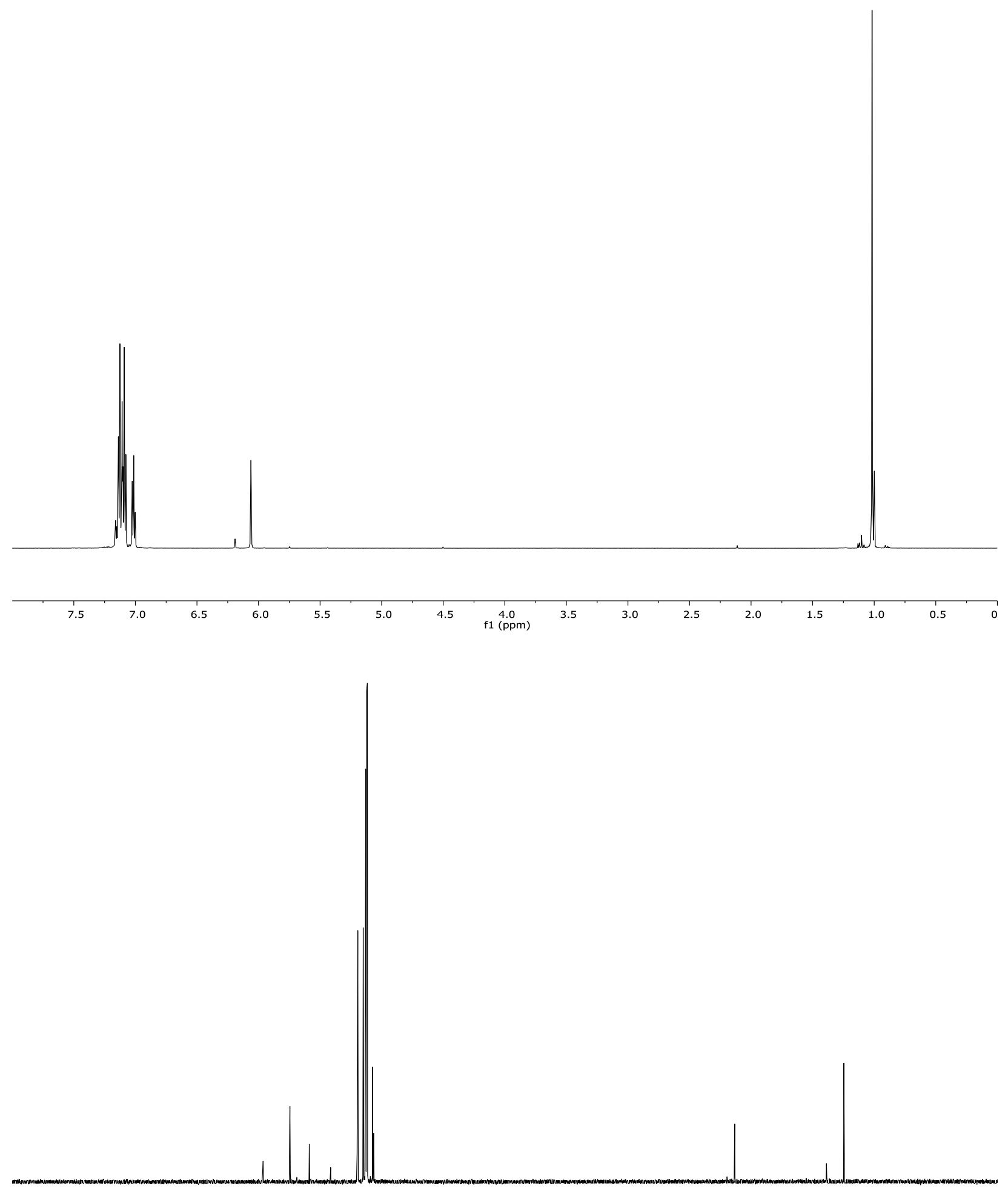


\subsubsection{3 ${ }^{1} \mathrm{H}$ NMR and ${ }^{13} \mathrm{C}$ Spectra of 26}
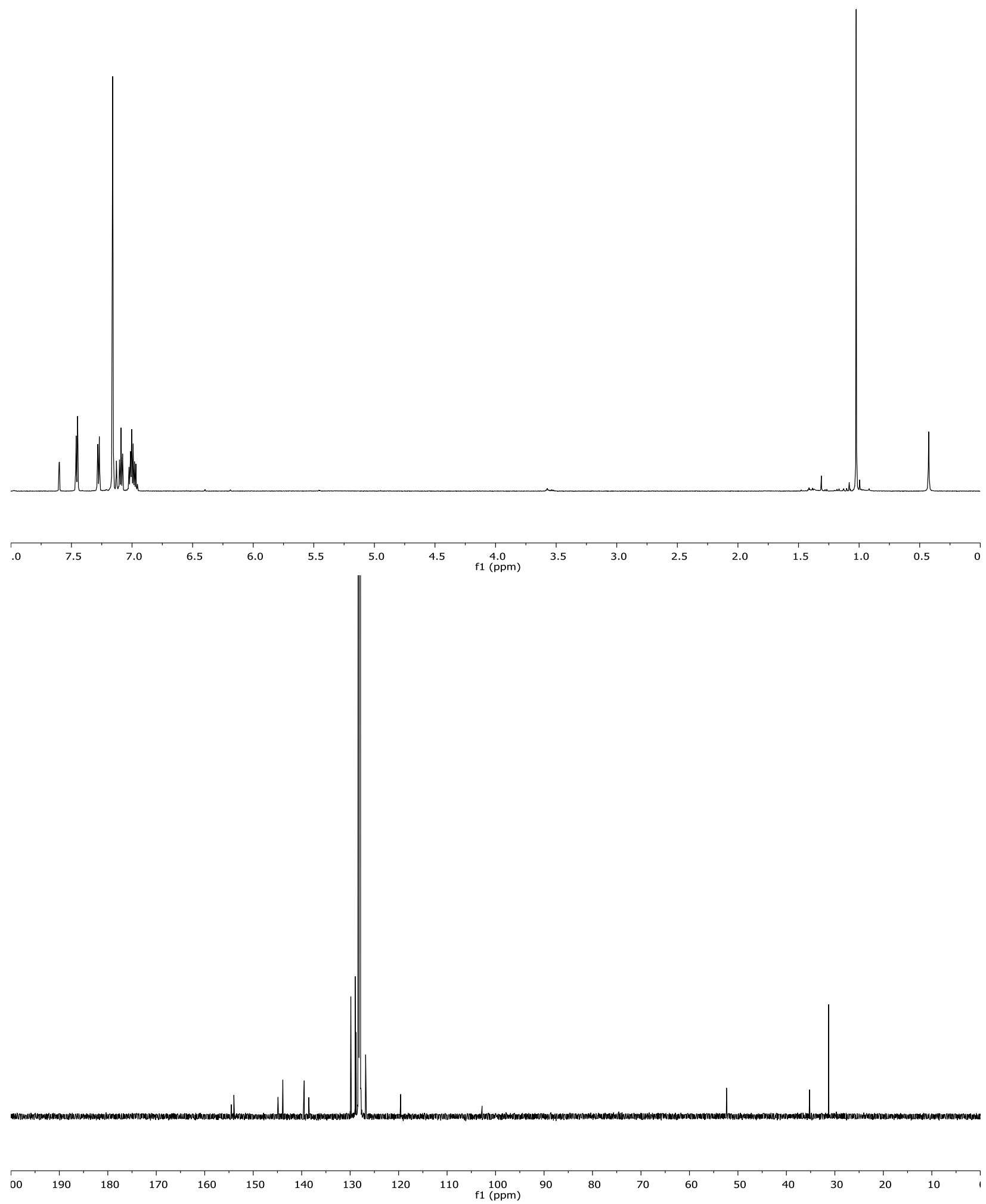


\subsection{Crystal Structure and Refinement Data}

Crystal structure refinement data is located on the following pages. Data collection SuperNova, Dual, $\mathrm{Cu}$ at zero, EOS, Program package WinGX , Abs. correction 'MULTISCAN' Refinement using SHELXL-2013 , Drawing using ORTEP-3 for Windows 

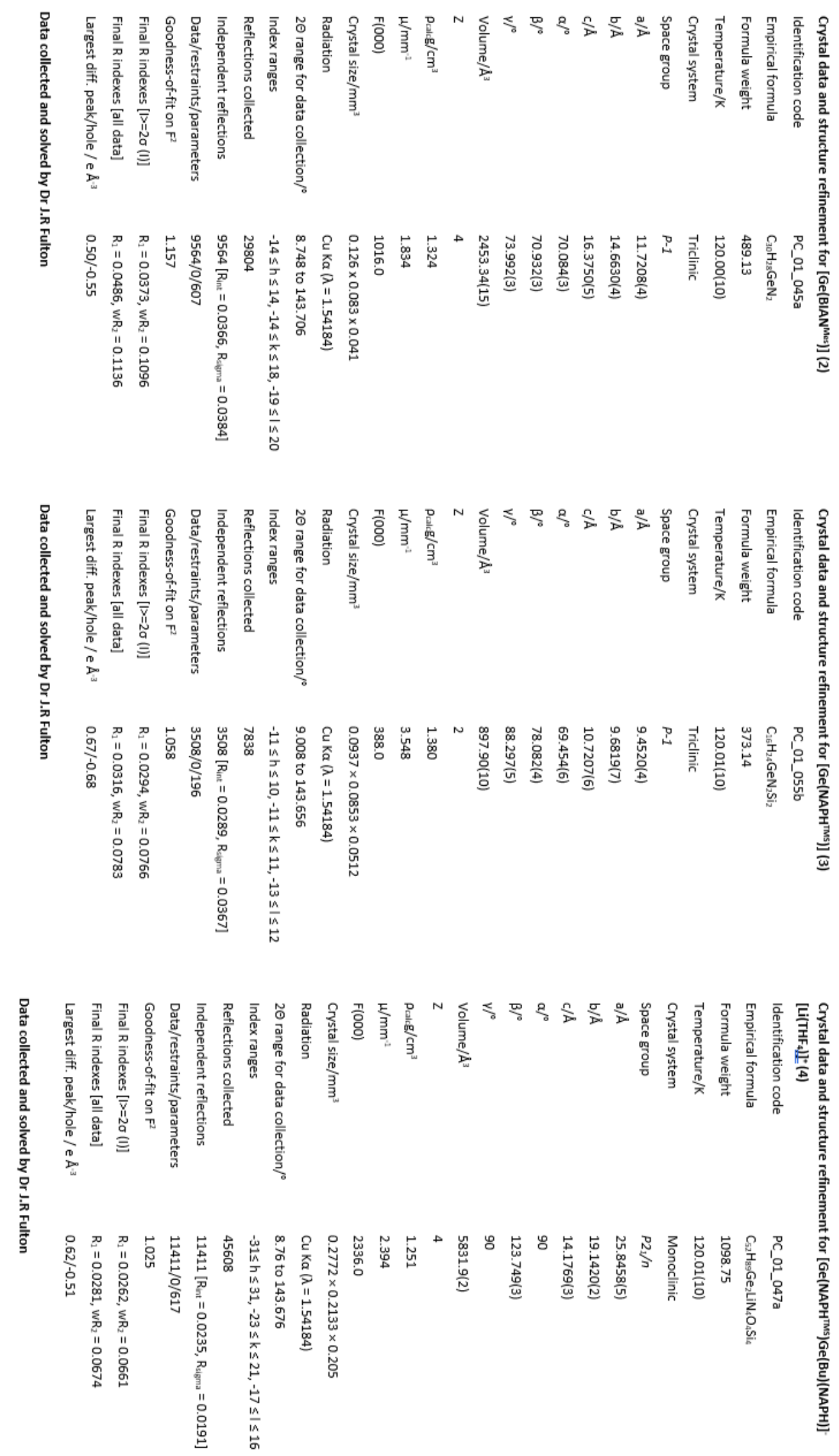

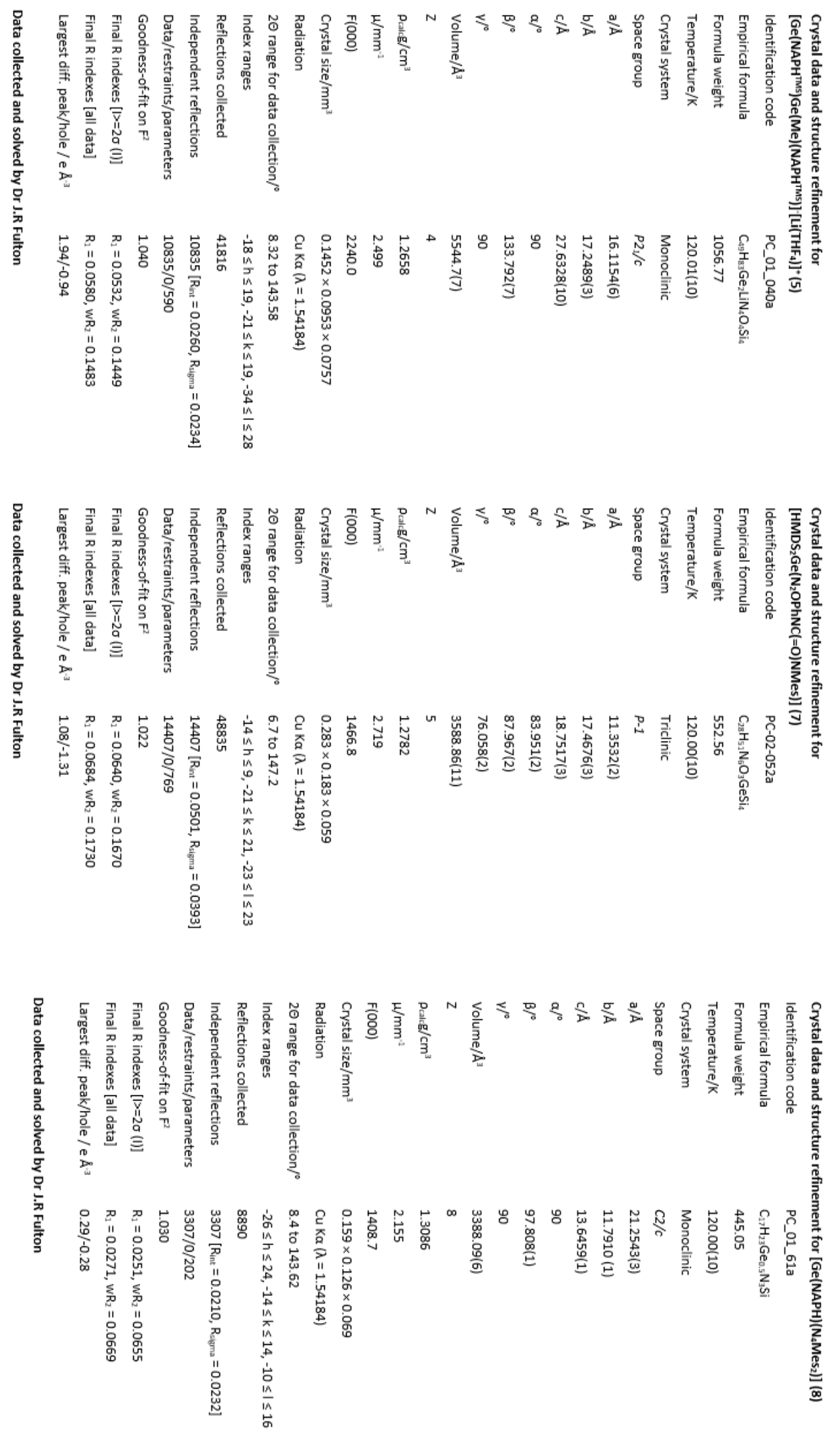

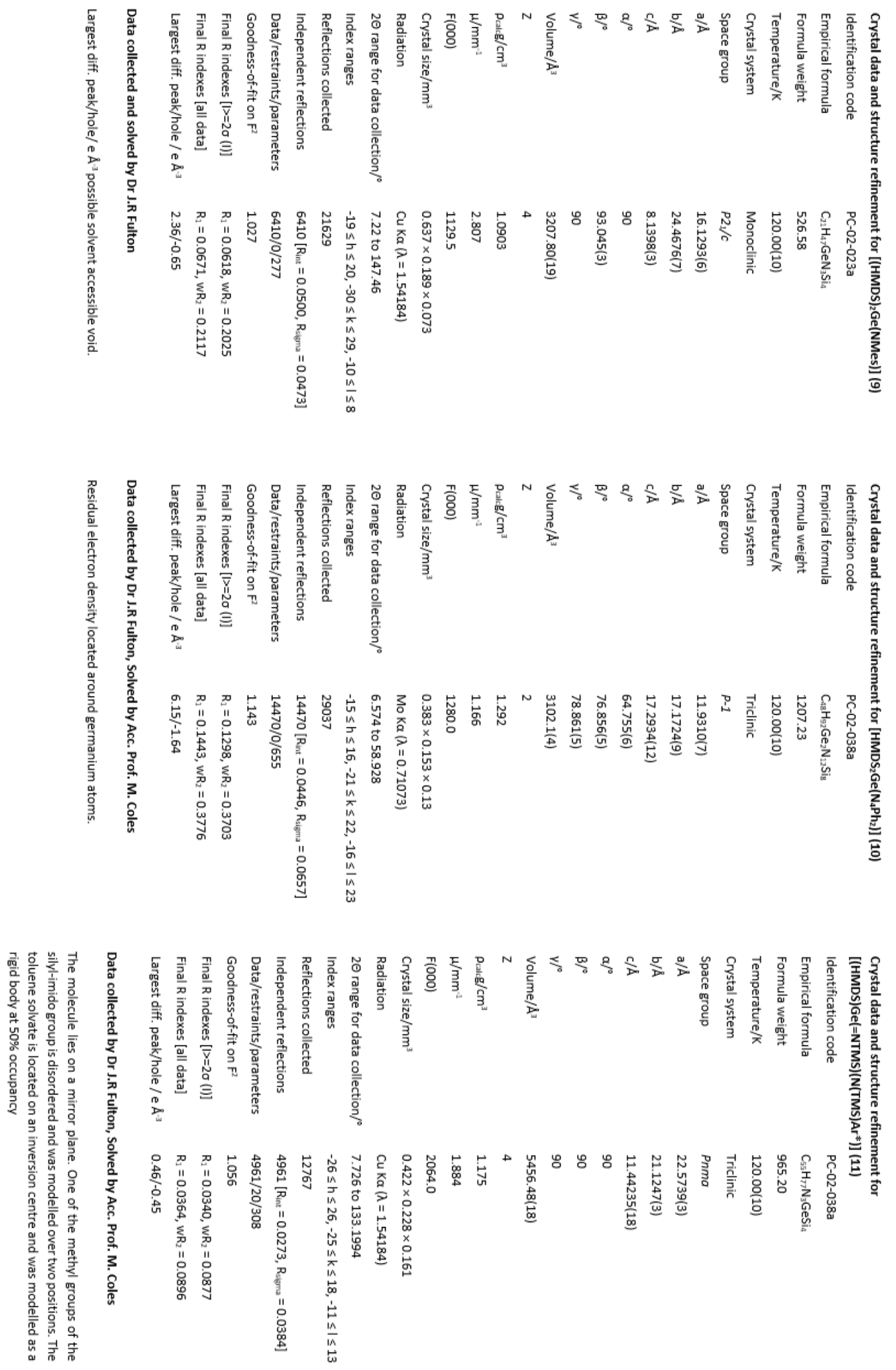

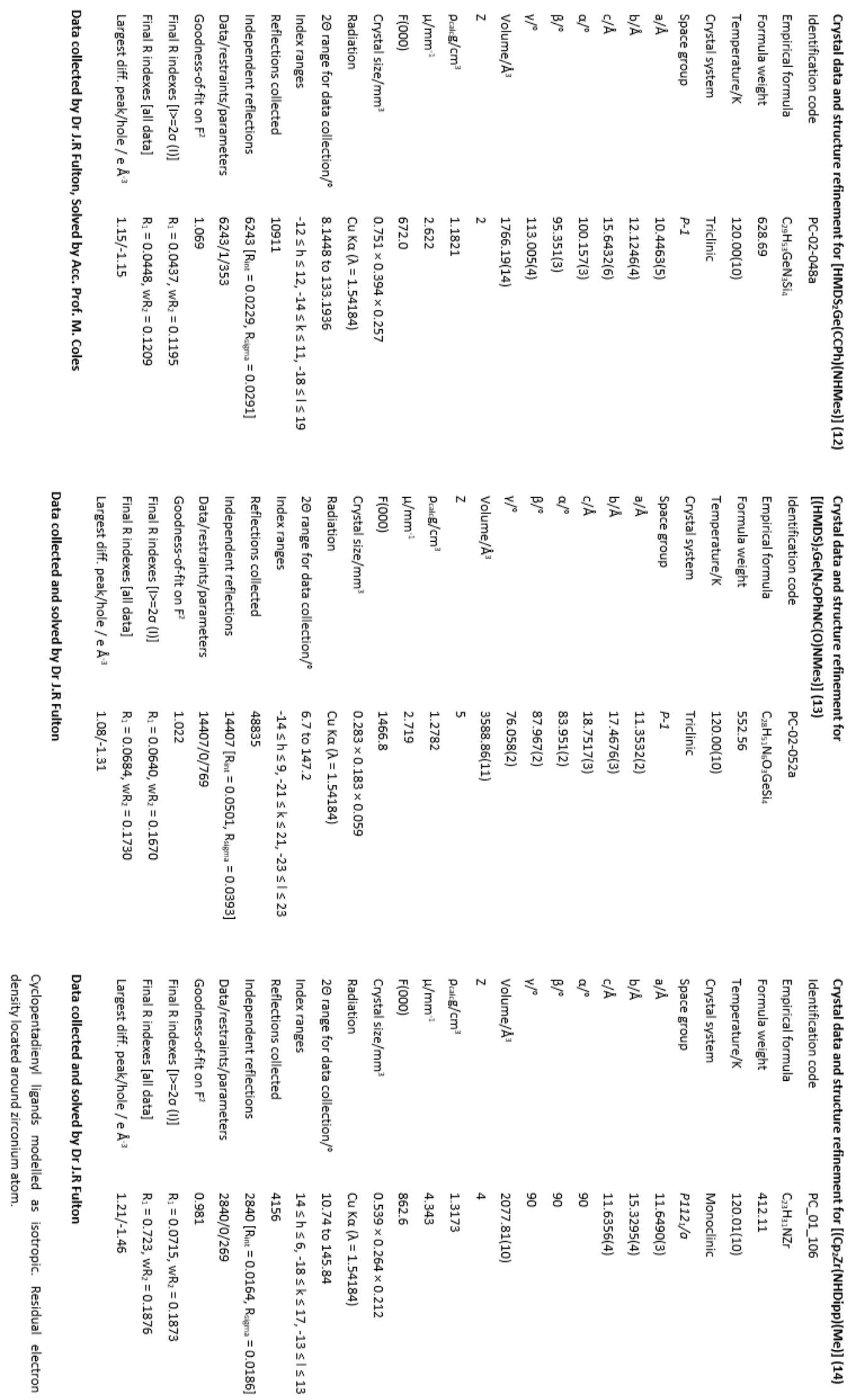

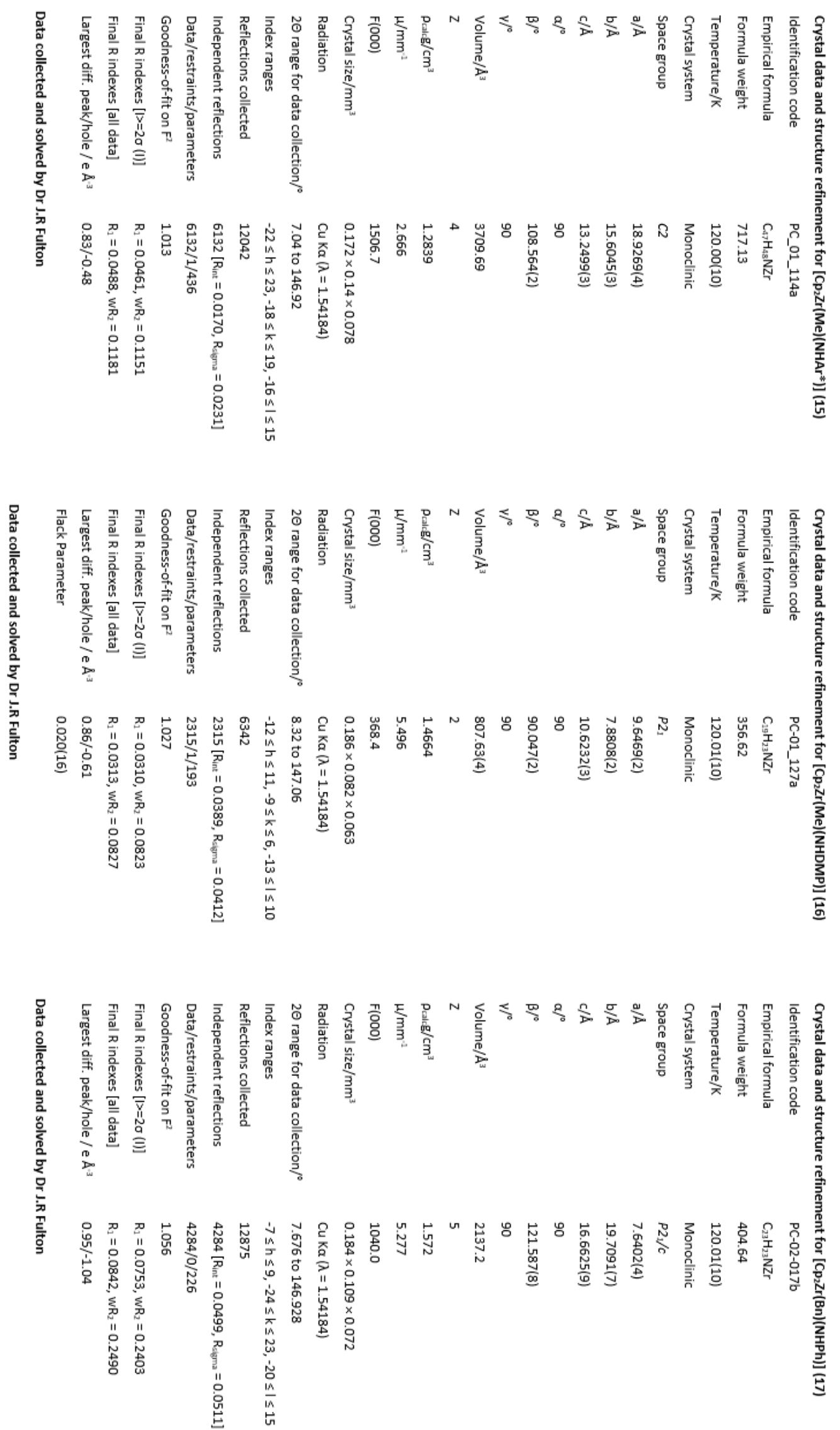

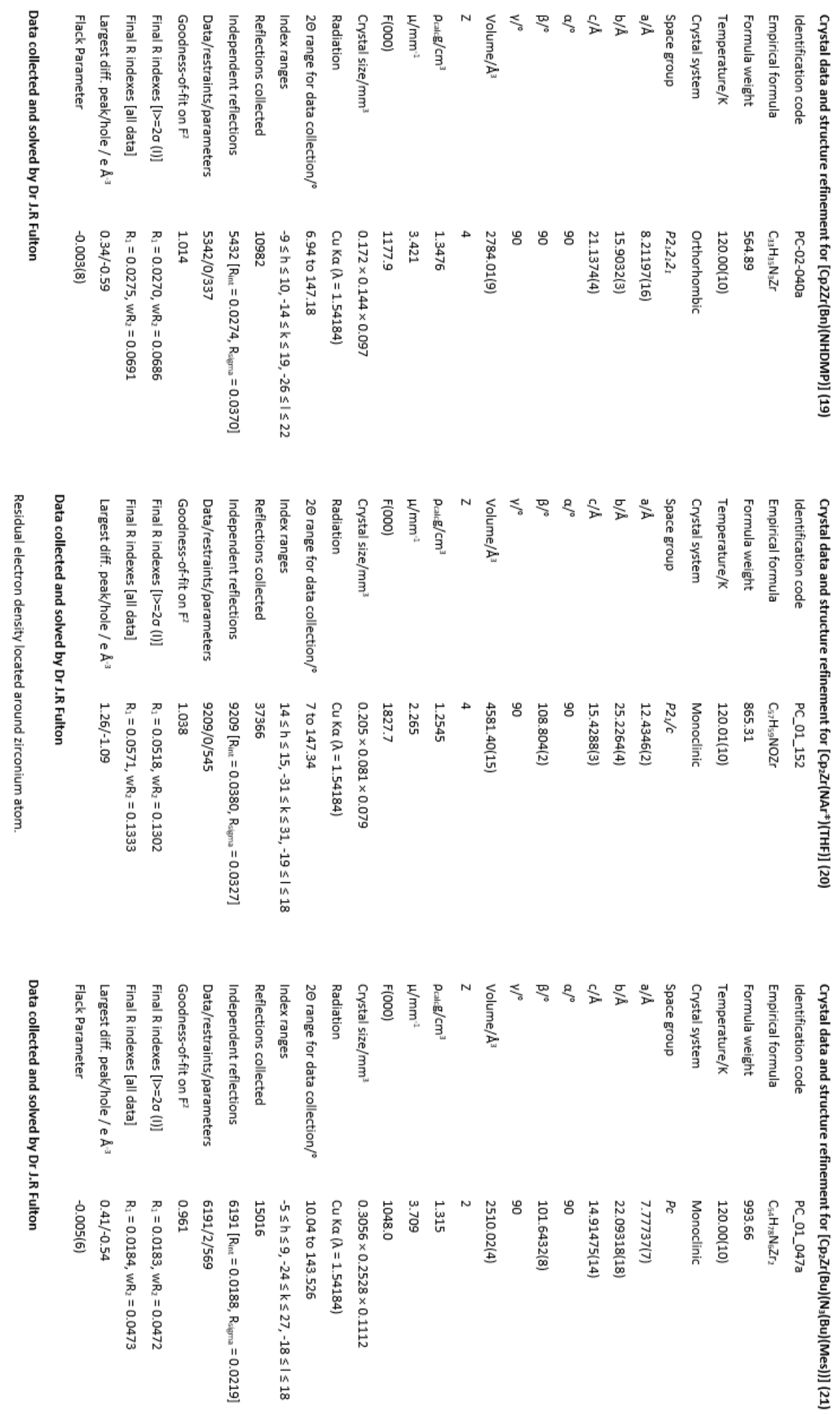


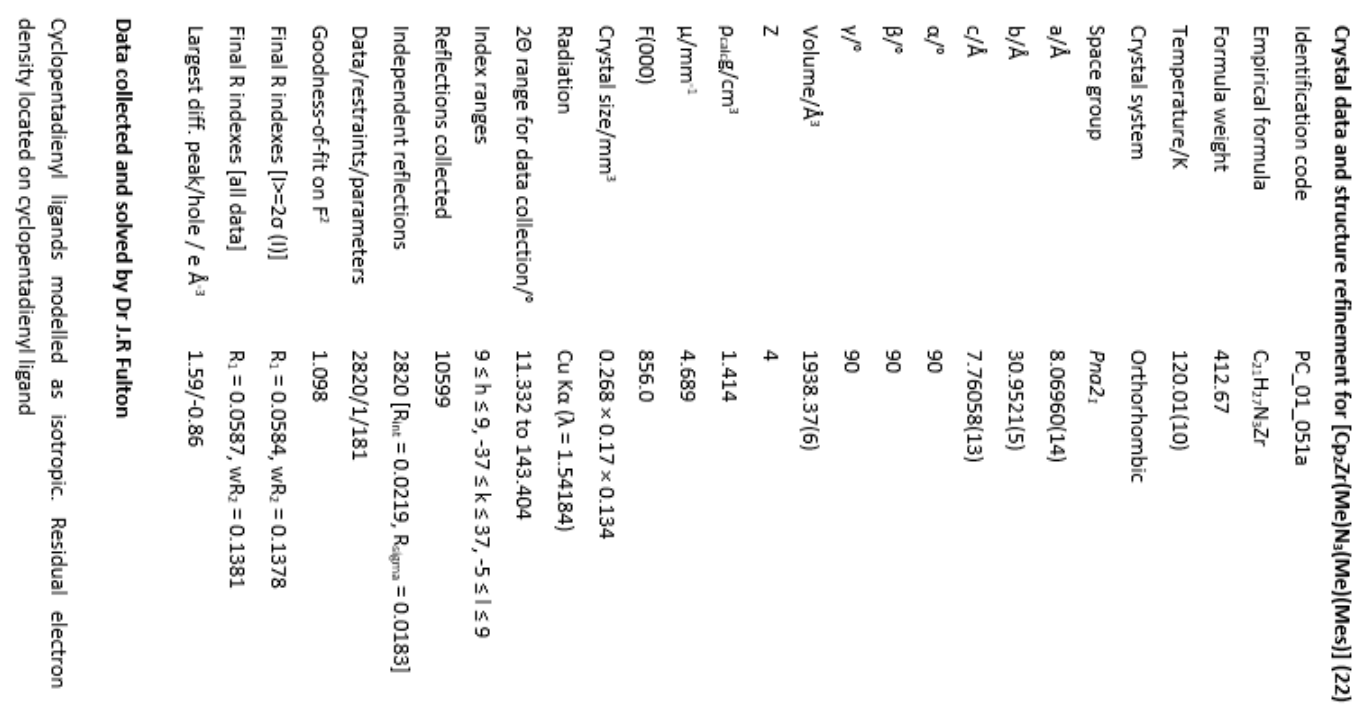

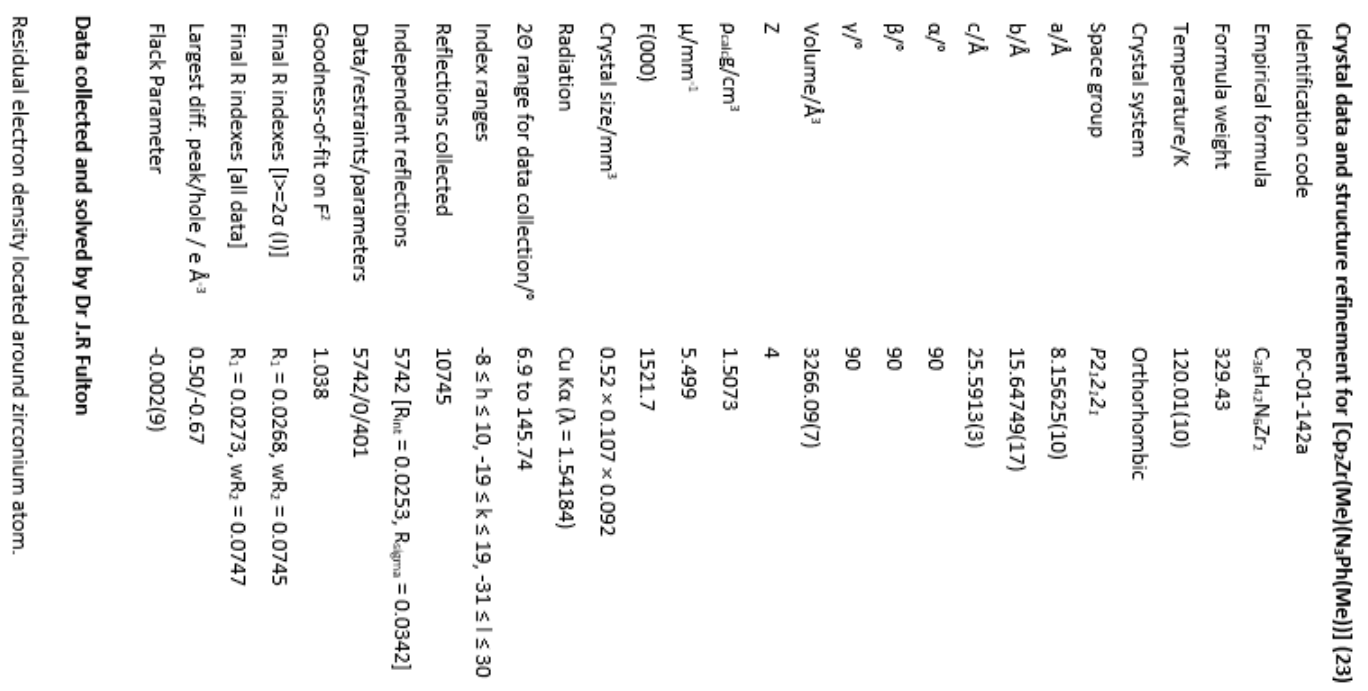

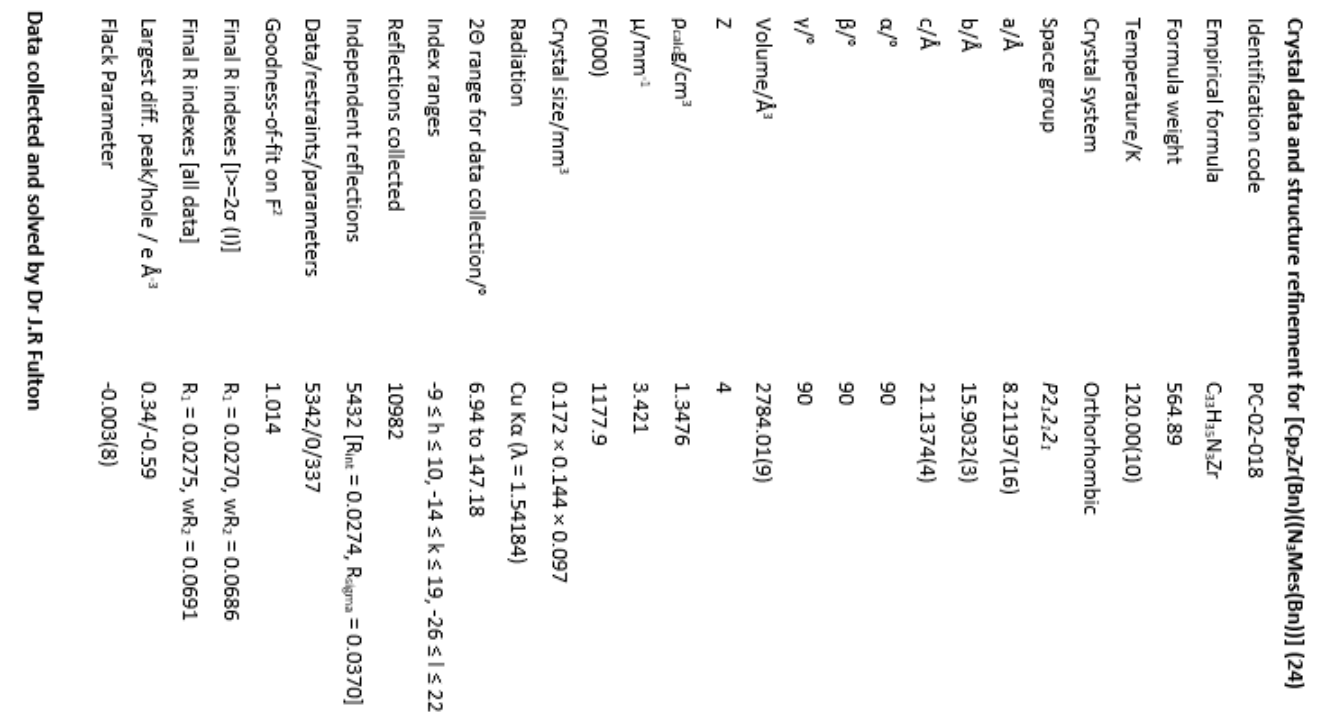



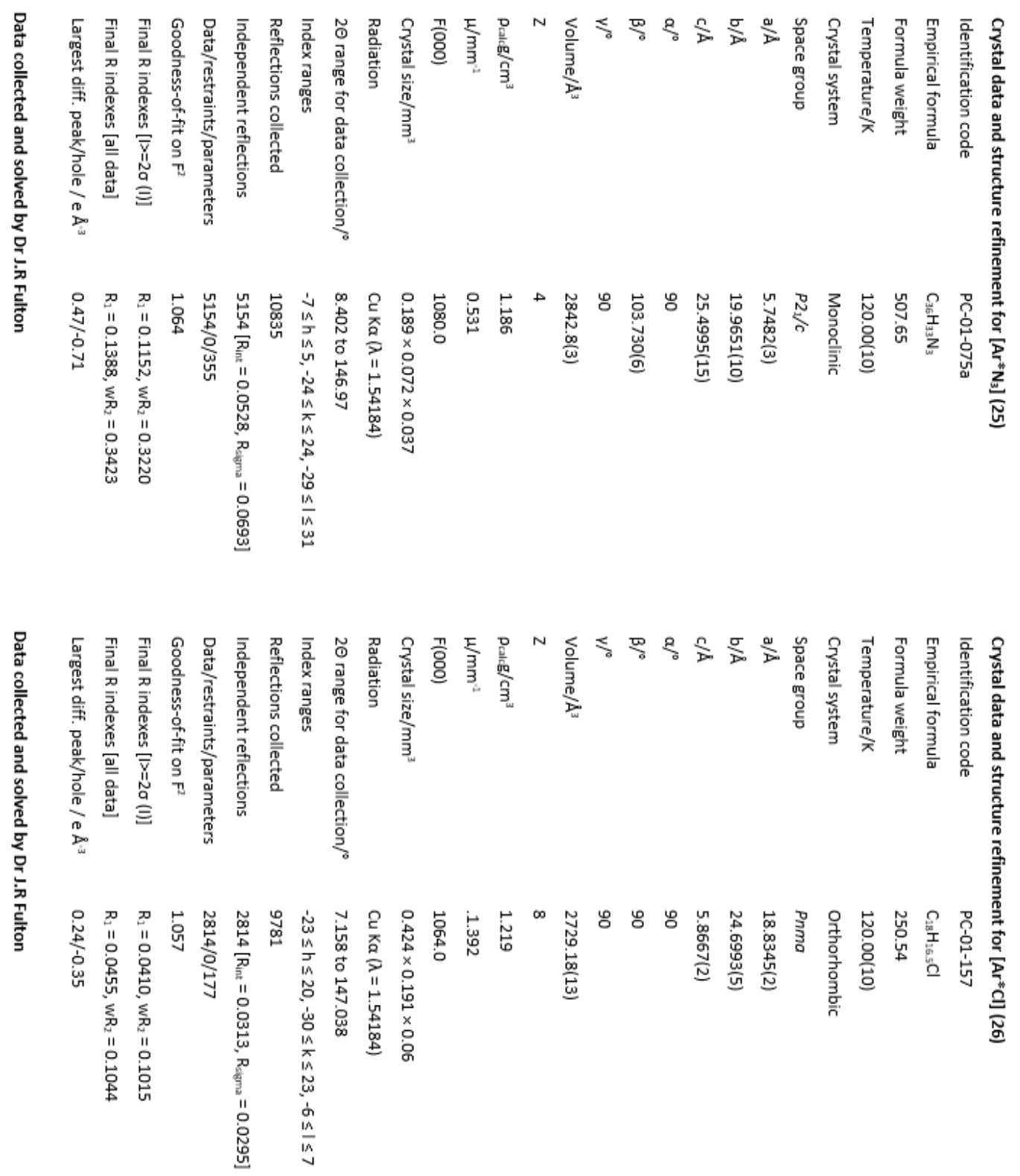


\subsection{Partial Charges for 4 and 5}

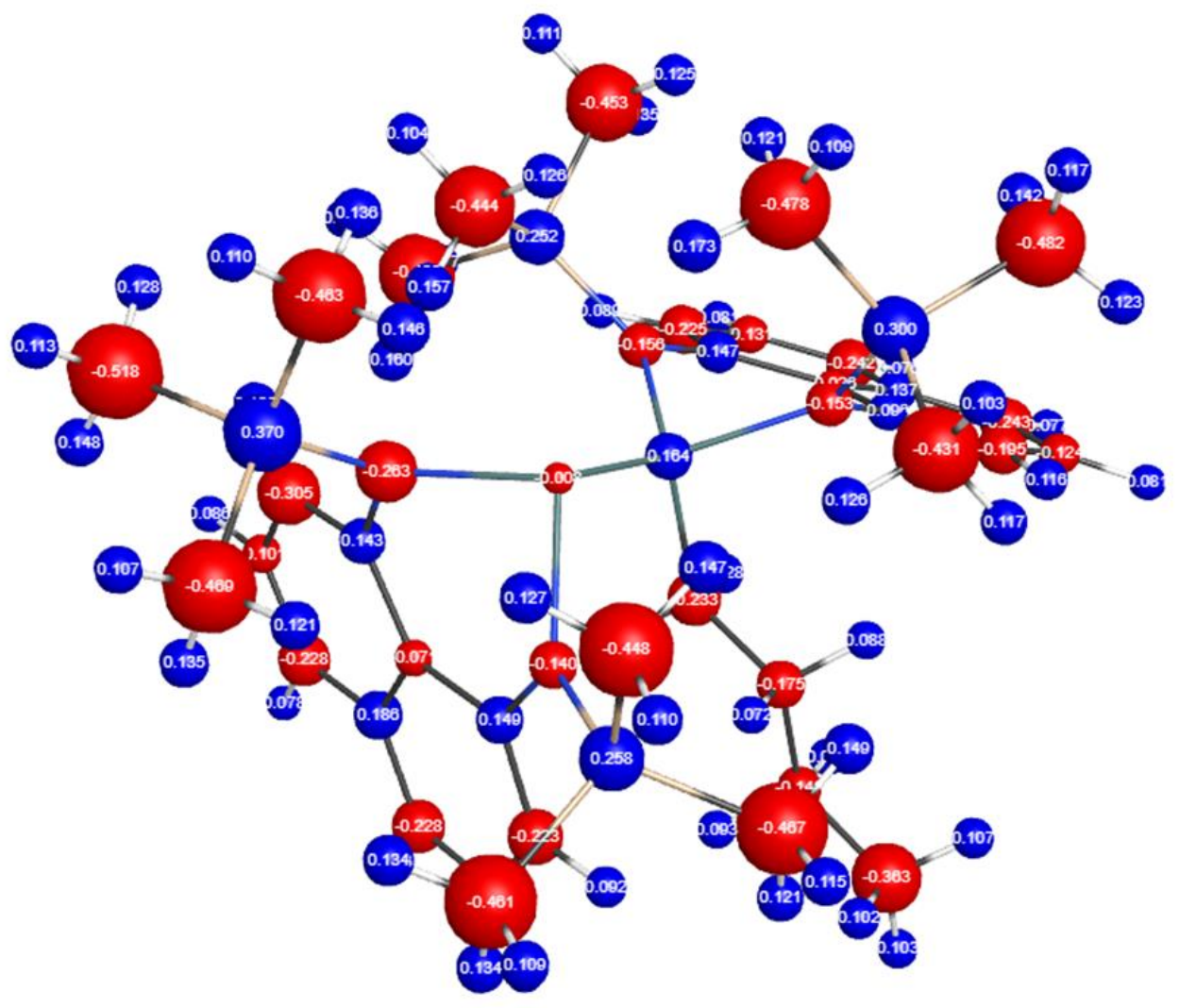

Figure 51: Partial charges calculated for 4 showing negative (red) and positive (blue) partial atom charges. 


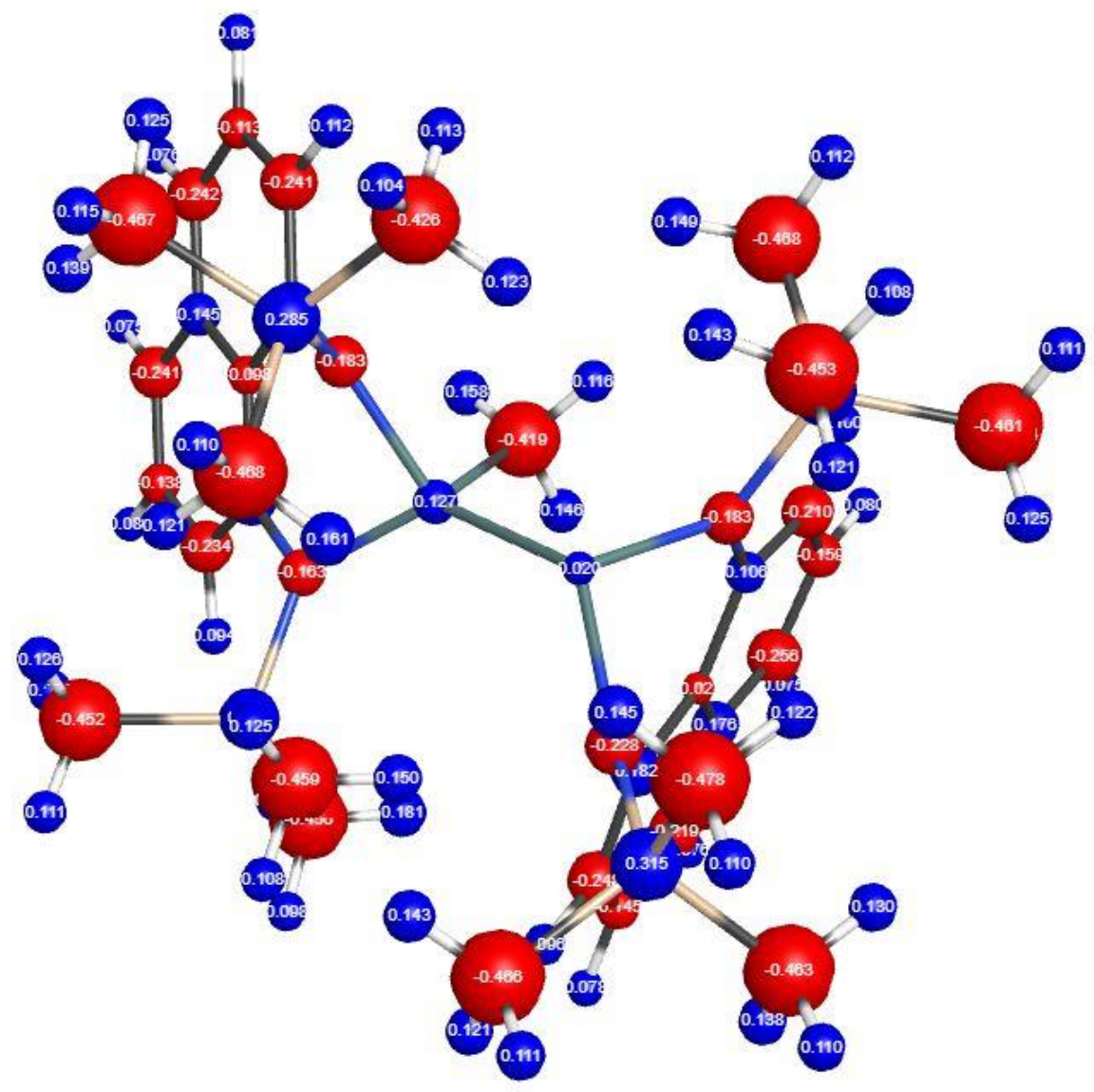

Figure 52: Partial charge calculation output of 5 showing negative (red) and positive (blue) partial atom charges.

\subsection{DFT Molecular Orbital Calculation Outputs for [Ge(HMDS) $\left.)_{2}\right]$}

Table 28: Molecular orbital energies of I.

HOMO-1 $\left(\mathrm{kJ} \mathrm{mol}^{-1}\right)$
HOMO $\left(\mathrm{kJ} \mathrm{mol}^{-1}\right)$
LUMO $\left(\mathrm{kJ} \mathrm{mol}^{-1}\right)$
LUMO+1 $\left(\mathrm{kJ} \mathrm{mol}^{-1}\right)$
HOMO-LUMO $\left(\mathrm{kJ} \mathrm{mol}^{-1}\right)$
HOMO-LUMO (eV)

[Ge(HMDS) $)_{2}$ ]

$-682.61$

$-585.47$

$-144.40$

68.26

441.07

4.57 


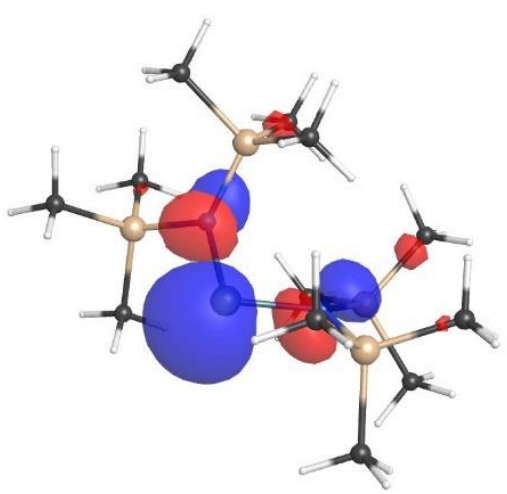

HOMO

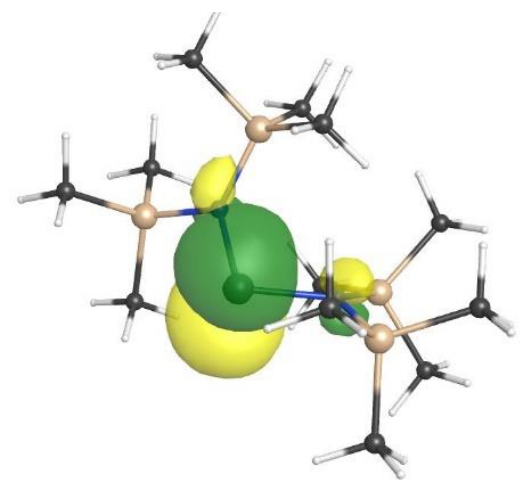

LUMO

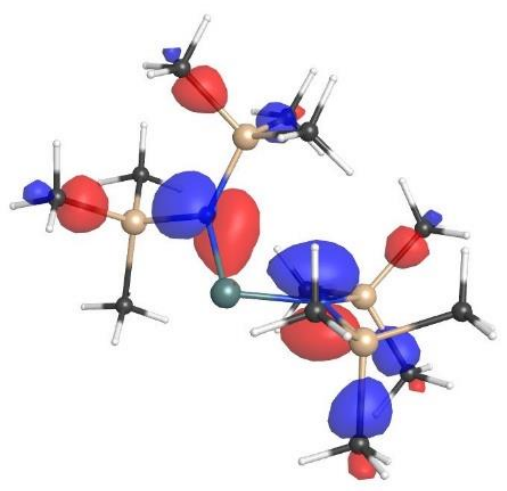

HOMO-1

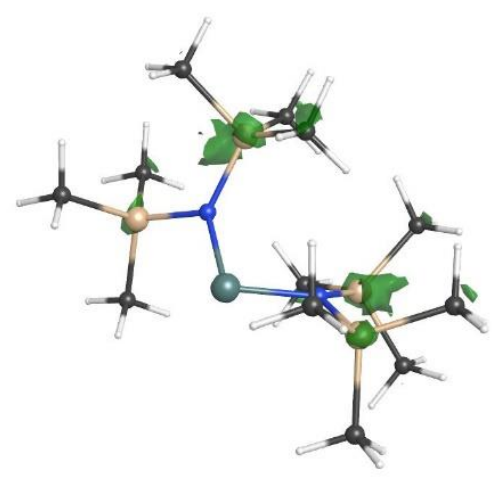

LUMO+1

Figure 53: Frontier molecular orbitals of [Ge(HMDS) $\left.)_{2}\right]$. 


\subsection{Crystal Structure Data for $\left[\mathrm{Ar}^{*} \mathrm{~N}_{3}\right]$}

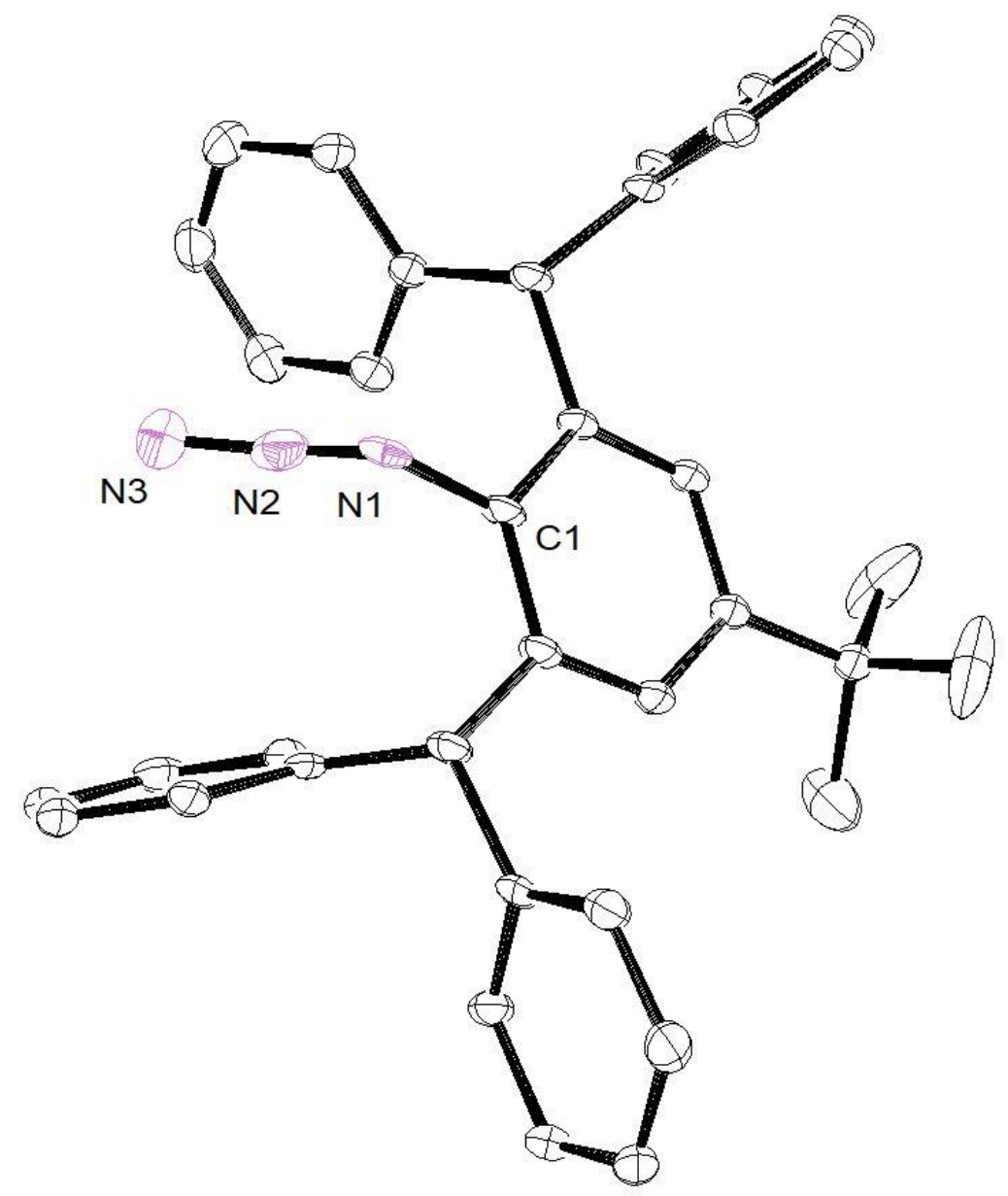

Figure 54: ORTEP representation of $25.30 \%$ Thermal ellipsoids. Hydrogens omitted for clarity.

Table 29: Selected bond lengths and angles for 25.

Bond Lengths (Å)

$\mathrm{N}(1)-\mathrm{N}(2)$

$\mathrm{N}(2)-\mathrm{N}(3)$

$N(1)-C(1)$
$1.133(7)$

$1.198(8)$

$1.485(6)$
Bond Angles ( ${ }^{\circ}$ )

$\mathrm{N}(1)-\mathrm{N}(2)-\mathrm{N}(3)$

169.9(6)

$N(2)-N(1)-C(1)$

$117.7(4)$ 


\subsection{Crystal Structure Data for $\left[\mathrm{Ar}^{*} \mathrm{Cl}\right]$}

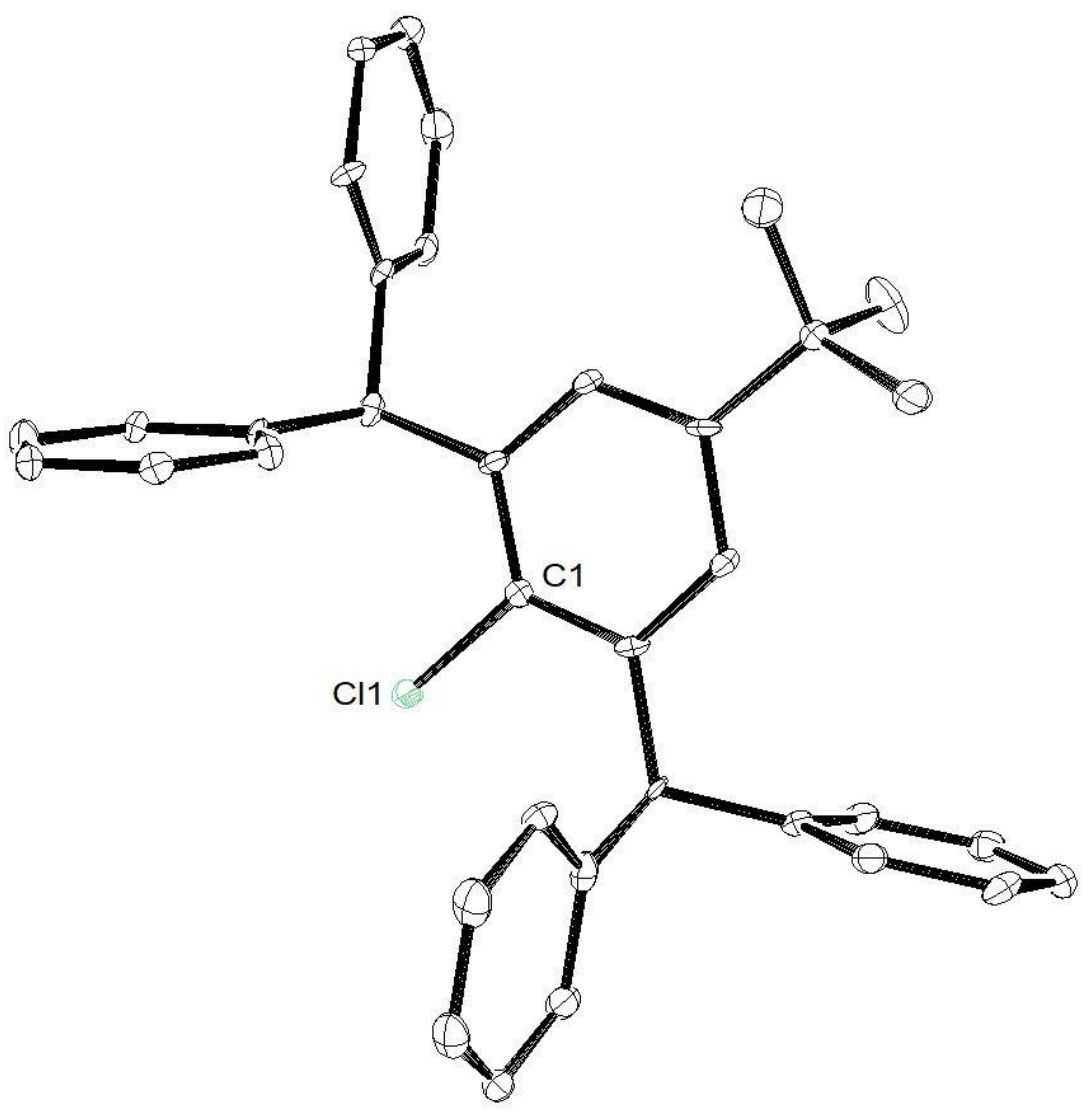

Figure 55: ORTEP representation of $26,30 \%$ thermal ellipsoids. Hydrogens omitted for clarity.

Table 30: Selected bond lengths and angles for 26 .

Bond Lengths ( $\AA$ )

$\mathrm{C}(1)-\mathrm{Cl}(1)$

1.748 


\subsection{Crystal Structure Diagram for $\left[\left(\mathrm{Cp}_{2} \mathrm{Zr}(\mathrm{NPh})\right)_{2}\right]$}

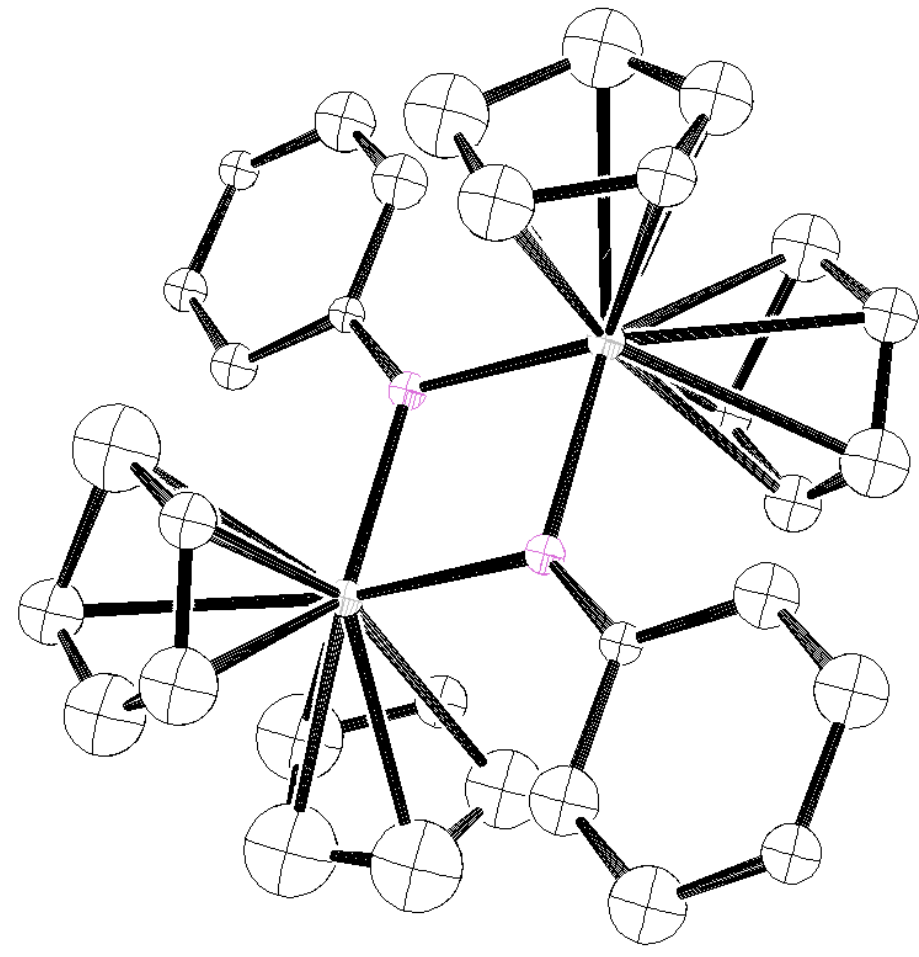

Figure 56: ORTEP diagram for $\left[\left(\mathrm{Cp}_{2} \mathrm{Zr}(\mathrm{NPh})\right)_{2}\right]$. Shown for connectivity purposes only. Isotropic model. 


\subsection{References:}

1. Baranger, A. M.; Hollander, F. J.; Bergman, R. G., Synthesis of an early-late heterobimetallic imido complex: cooperative reactivity with nitrogen-, oxygen-, and sulfur-hydrogen bonds and formation of a novel aminophosphine complex. Journal of the American Chemical Society 1993, 115 (17), 7890-7891.

2. Stephan, D. W., Frustrated Lewis pairs: a new strategy to small molecule activation and hydrogenation catalysis. Dalton Transactions 2009, (17), 3129-3136.

3. Power, P. P., Persistent and stable radicals of the heavier main group elements and related species. Chemical reviews 2003, 103 (3), 789-810.

4. Fischer, R. C.; Power, P. P., $\pi$-Bonding and the lone pair effect in multiple bonds involving heavier main group elements: developments in the new millennium. Chemical reviews 2010, 110 (7), 3877-3923.

5. Al-Rafia, S. I.; Lummis, P. A.; Ferguson, M. J.; McDonald, R.; Rivard, E., Low-Coordinate Germylene and Stannylene Heterocycles featuring Sterically Tunable Bis (amido) silyl Ligands. Inorganic chemistry 2010, 49 (20), 9709-9717.

6. Hadlington, T. J.; Abdalla, J. A.; Tirfoin, R.; Aldridge, S.; Jones, C., Stabilization of a twocoordinate, acyclic diaminosilylene (ADASi): completion of the series of isolable diaminotetrylenes: $\mathrm{E}\left(\mathrm{NR}_{2}\right)_{2}$ ( $\mathrm{E}=$ group 14 element). Chemical Communications 2016, 52 (8), 17171720.

7. Protchenko, A. V.; Bates, J. I.; Saleh, L. M.; Blake, M. P.; Schwarz, A. D.; Kolychev, E. L.; Thompson, A. L.; Jones, C.; Mountford, P.; Aldridge, S., Enabling and Probing Oxidative Addition and Reductive Elimination at a Group 14 Metal Center: Cleavage and Functionalization of E-H Bonds by a Bis (boryl) stannylene. Journal of the American Chemical Society 2016, 138 (13), 4555-4564.

8. Protchenko, A. V.; Birjkumar, K. H.; Dange, D.; Schwarz, A. D.; Vidovic, D.; Jones, C.; Kaltsoyannis, N.; Mountford, P.; Aldridge, S., A stable two-coordinate acyclic silylene. Journal of the American Chemical Society 2012, 134 (15), 6500-6503.

9. Negishi, E.-i.; Takahashi, T., Patterns of stoichiometric and catalytic reactions of organozirconium and related complexes of synthetic interest. Accounts of chemical research 1994, 27 (5), 124130.

10. Lappert, M. F.; Rowe, R. S., The role of group 14 element carbene analogues in transition metal chemistry. Coordination Chemistry Reviews 1990, 100, 267-292.

11. Schwerdtfeger, P.; Heath, G. A.; Dolg, M.; Bennett, M. A., Low valencies and periodic trends in heavy element chemistry. A theoretical study of relativistic effects and electron correlation effects in Group 13 and Period 6 hydrides and halides. Journal of the American Chemical Society 1992, 114 (19), 7518-7527.

12. Drago, R. S., Thermodynamic Evaluation of the Inert Pair Effect. The Journal of Physical Chemistry 1958, 62 (3), 353-357.

13. Lui, M. W.; Merten, C.; Ferguson, M. J.; McDonald, R.; Xu, Y.; Rivard, E., Contrasting reactivities of silicon and germanium complexes supported by an $\mathrm{N}$-heterocyclic guanidine ligand. Inorganic chemistry 2015, 54 (4), 2040-2049.

14. Lee, V. Y.; Sekiguchi, A., Organometallic compounds of low-coordinate Si, Ge, Sn and Pb: from phantom species to stable compounds. John Wiley \& Sons: 2011.

15. Bourissou, D.; Guerret, O.; Gabbai, F. P.; Bertrand, G., Stable carbenes. Chemical Reviews 2000, 100 (1), 39-92. 
16. Usher, M.; Protchenko, A. V.; Rit, A.; Campos, J.; Kolychev, E. L.; Tirfoin, R.; Aldridge, S., A Systematic Study of Structure and E- H Bond Activation Chemistry by Sterically Encumbered Germylene Complexes. Chemistry-A European Journal 2016, 22 (33), 11685-11698.

17. Carter, E. A.; Goddard III, W. A., Relation between singlet-triplet gaps and bond energies. The Journal of Physical Chemistry 1986, 90 (6), 998-1001.

18. Baird, N. C.; Taylor, K. F., Multiplicity of the ground state and magnitude of the T1-S0 gap in substituted carbenes. Journal of the American Chemical Society 1978, 100 (5), 1333-1338.

19. Harris, D. H.; Lappert, M. F., Monomeric, volatile bivalent amides of group IV B elements, $\mathrm{M}\left(\mathrm{NR}_{2}\right)_{2}$ and $\mathrm{M}\left(\mathrm{NR}_{1} \mathrm{R}_{2}\right)_{2} \mathrm{Ge}, \mathrm{Sn}$, or Pb. Journal of the Chemical Society, Chemical Communications 1974, (21), 895-896.

20. Petz, W., Transition-metal complexes with derivatives of divalent silicon, germanium, tin, and lead as ligands. Chemical Reviews 1986, 86 (6), 1019-1047.

21. Woodul, W. D.; Carter, E.; Müller, R.; Richards, A. F.; Stasch, A.; Kaupp, M.; Murphy, D. M.; Driess, M.; Jones, C., A neutral, monomeric germanium (I) radical. Journal of the American Chemical Society 2011, 133 (26), 10074-10077.

22. Krupski, S.; Pöttgen, R.; Schellenberg, I.; Hahn, F. E., Benzannulated N-heterocyclic germylenes and stannylenes with sterically demanding N, N'-substituents. Dalton Transactions 2014, 43 (1), 173-181.

23. Pfeiffer, J.; Maringgele, W.; Noltemeyer, M.; Meller, A., Reaktionen von germylenen mit aziden: iminogermane, azidogermane, tetrazagermole und hexaazadigermadispirododecane. European Journal of Inorganic Chemistry 1989, 122 (2), 245-252.

24. Mizuhata, Y.; Sasamori, T.; Tokitoh, N., Stable heavier carbene analogues. Chemical reviews 2009, 109 (8), 3479-3511.

25. Fedushkin, I. L.; Skatova, A. A.; Chudakova, V. A.; Khvoinova, N. M.; Baurin, A. Y.; Dechert, S.; Hummert, M.; Schumann, H., Stable germylenes derived from 1, 2-bis (arylimino) acenaphthenes. Organometallics 2004, 23 (15), 3714-3718.

26. Bazinet, P.; Yap, G. P.; Richeson, D. S., Synthesis and properties of a germanium (II) metalloheterocycle derived from 1, 8-di (isopropylamino) naphthalene. A novel ligand leading to formation of $\mathrm{Ni}\left\{\mathrm{Ge}\left[\left({ }^{(} \mathrm{PrN}\right)_{2} \mathrm{C}_{10} \mathrm{H}_{6}\right]\right\}$ 4. Journal of the American Chemical Society 2001, 123 (45), 11162-11167.

27. Stanciu, C.; Richards, A. F.; Stender, M.; Olmstead, M. M.; Power, P. P., New terphenylphenoxides of group 13 and 14 elements. Polyhedron 2006, 25 (2), 477-483.

28. Simons, R. S.; Pu, L.; Olmstead, M. M.; Power, P. P., Synthesis and Characterization of the Monomeric Diaryls $\mathrm{M}\left\{\mathrm{C}_{6} \mathrm{H}_{3}-2,6-\mathrm{Mes}_{2}\right\}_{2}\left(\mathrm{M}=\mathrm{Ge}\right.$, Sn, or $\mathrm{Pb}$; Mes= 2, 4, 6- $\mathrm{Me}_{3} \mathrm{C}_{6} \mathrm{H}_{2}$ ) and Dimeric Aryl- Metal Chlorides $\left[\mathrm{M}(\mathrm{Cl})\left\{\mathrm{C}_{6} \mathrm{H}_{3}-2,6-\mathrm{Mes}_{2}\right\}\right]_{2}$ ( $\mathrm{M}=\mathrm{Ge}$ or Sn). Organometallics 1997, 16 (9), 1920-1925.

29. Izod, K.; Rayner, D. G.; El-Hamruni, S. M.; Harrington, R. W.; Baisch, U., Stabilization of a Diphosphagermylene through $\mathrm{p} \pi-\mathrm{p} \pi$ Interactions with a Trigonal-Planar Phosphorus Center. Angewandte Chemie 2014, 126 (14), 3710-3714.

30. Katir, N.; Matioszek, D.; Ladeira, S.; Escudié, J.; Castel, A., Stable N-Heterocyclic Carbene Complexes of Hypermetallyl Germanium (II) and Tin (II) Compounds. Angewandte Chemie International Edition 2011, 50 (23), 5352-5355.

31. Ghadwal, R. S.; Azhakar, R.; Roesky, H. W., Dichlorosilylene: a high temperature transient species to an indispensable building block. Accounts of chemical research 2012, 46 (2), 444-456.

32. Álvarez-Rodríguez, L.; Cabeza, J. A.; García-Álvarez, P.; Polo, D., The transition-metal chemistry of amidinatosilylenes,-germylenes and-stannylenes. Coordination Chemistry Reviews 2015, 300, $1-28$. 
33. Simons, R. S.; Pu, L.; Olmstead, M. M.; Power, P. P., Synthesis and characterization of the monomeric diaryls $\mathrm{M}\left\{\mathrm{C}_{6} \mathrm{H}_{3}-2,6-\mathrm{Mes}_{2}\right\}_{2}\left(\mathrm{M}=\mathrm{Ge}\right.$, $\mathrm{Sn}$, or $\mathrm{Pb}$; Mes= 2, 4, 6- $\left.-\mathrm{Me}_{3} \mathrm{C}_{6} \mathrm{H}_{2}\right)$ and dimeric aryl-metal chlorides $\left[\mathrm{M}(\mathrm{Cl})\left\{\mathrm{C}_{6} \mathrm{H}_{3}-2,6-\mathrm{Mes}_{2}\right\}\right]_{2}(\mathrm{M}=\mathrm{Ge}$ or Sn). Organometallics 1997, 16 (9), 19201925.

34. Snow, J. T.; Murakami, S.; Masamune, S.; Williams, D. J., Synthesis and characterization of tetrakis (2, 6-diethylphenyl) digermene. Tetrahedron letters 1984, 25 (38), 4191-4194.

35. Hurni, K. L.; Rupar, P. A.; Payne, N. C.; Baines, K. M., On the synthesis, structure, and reactivity of tetramesityldigermene. Organometallics 2007, 26 (23), 5569-5575.

36. Schäfer, H.; Saak, W.; Weidenbruch, M., Azadigermiridines by Addition of Diazomethane or Trimethylsilyldiazomethane to a Digermene. Organometallics 1999, 18 (16), 3159-3163.

37. Jana, A.; Objartel, I.; Roesky, H. W.; Stalke, D., Cleavage of a N-H Bond of Ammonia at Room Temperature by a Germylene. Inorganic chemistry 2008, 48 (3), 798-800.

38. Lu, E.; Li, Y.; Chen, Y., A scandium terminal imido complex: synthesis, structure and DFT studies. Chemical Communications 2010, 46 (25), 4469-4471.

39. Geer, A. M.; Tejel, C.; López, J. A.; Ciriano, M. A., Terminal Imido Rhodium Complexes. Angewandte Chemie International Edition 2014, 53 (22), 5614-5618.

40. Meller, A.; Ossig, G.; Maringgele, W.; Stalke, D.; Herbst-Irmer, R.; Freitag, S.; Sheldrick, G. M., Synthesis and X-ray crystal structure of germaimines. Journal of the Chemical Society, Chemical Communications 1991, (16), 1123-1124.

41. Ando, W.; Ohtaki, T.; Kabe, Y., Synthesis, structure, and photoreaction of bis (germanimines). Organometallics 1994, 13 (2), 434-435.

42. Trinquier, G.; Barthelat, J. C.; Satge, J., Double bonds vs. carbene-like unsaturations in germanium intermediates. Journal of the American Chemical Society 1982, 104 (22), 5931-5936.

43. Schneider, J.; Krebs, K. M.; Freitag, S.; Eichele, K.; Schubert, H.; Wesemann, L., Intramolecular Tetrylene Lewis Adducts: Synthesis and Reactivity. Chemistry-A European Journal 2016, 22 (28), 9812-9826.

44. Preut, H.; Obloh, R. C.; Neumann, W. P., 1, 3-Di-tert-butyl-2, 2-dimethyl-5, 8-diphenyl-1, 3, 5, 6, 7, 8-hexaaza-2-sila-4-germaspiro [3.4] oct-6-ene. Acta Crystallographica Section C: Crystal Structure Communications 1988, 44 (3), 574-575.

45. Leung, W.-P.; Chiu, W.-K.; Mak, T. C., Reactivity of Pyridyl-1-azaallyl Germanium (I) Dimer: Synthesis of a Digermahydrazine Derivative and an Iron-Coordinated Germanium (I) Dimer. Organometallics 2013, 33 (1), 225-230.

46. Li, J.; Schenk, C.; Goedecke, C.; Frenking, G.; Jones, C., A digermyne with a Ge-Ge single bond that activates dihydrogen in the solid state. Journal of the American Chemical Society 2011, 133 (46), 18622-18625.

47. Khan, S.; Gopakumar, G.; Thiel, W.; Alcarazo, M., Stabilization of a Two-Coordinate [GeCl]+ Cation by Simultaneous $\sigma$ and $\pi$ Donation from a Monodentate Carbodiphosphorane. Angewandte Chemie International Edition 2013, 52 (21), 5644-5647.

48. Al-Rafia, S.; Momeni, M. R.; McDonald, R.; Ferguson, M. J.; Brown, A.; Rivard, E., Controlled growth of dichlorogermanium oligomers from lewis basic hosts. Angewandte Chemie International Edition 2013, 52 (25), 6390-6395.

49. Walsh, P. J.; Hollander, F. J.; Bergman, R. G., Generation, alkyne cycloaddition, arene carbonhydrogen activation, nitrogen-hydrogen activation and dative ligand trapping reactions of the first monomeric imidozirconocene $\left(\mathrm{Cp}_{2} \mathrm{Zr}=\mathrm{NR}\right)$ complexes. Journal of the American Chemical Society 1988, 110 (26), 8729-8731.

50. Walsh, P. J.; Hollander, F. J.; Bergman, R. G., Monomeric and dimeric zirconocene imido compounds: synthesis, structure, and reactivity. Organometallics 1993, 12 (9), 3705-3723. 
51. Zuckerman, R. L.; Krska, S. W.; Bergman, R. G., Zirconium-mediated metathesis of imines: A study of the scope, longevity, and mechanism of a complicated catalytic system. Journal of the American Chemical Society 2000, 122 (5), 751-761.

52. Duncan, A. P.; Bergman, R. G., Selective transformations of organic compounds by imidozirconocene complexes. The Chemical Record 2002, 2 (6), 431-445.

53. Wigley, D. E., Organoimido complexes of the transition metals. Prog. Inorg. Chem 1994, 42, 239482.

54. Zuckerman, R. L.; Bergman, R. G., Structural factors that influence the course of overall [2+2] cycloaddition reactions between imidozirconocene complexes and heterocumulenes. Organometallics 2000, 19 (23), 4795-4809.

55. Meyer, K. E.; Walsh, P. J.; Bergman, R. G., A Mechanistic Study of the CycloadditionCycloreversion Reactions of Zirconium-Imido Complex $\mathrm{Cp}_{2} \mathrm{Zr}\left(=\mathrm{N}^{t} \mathrm{Bu}\right)(\mathrm{THF})$ with Organic Imines and Organic Azides. Journal of the American Chemical Society 1995, 117 (3), 974-985.

56. Soon, W. L., Vanadium Tetrazene Complex: Synthesis of Chlorophosphine (1, 4diphenyltetrazenido) vanadium (III),. Bulletin of the Korean Chemical Society 1993, 14 (1), 1-2.

57. Trogler, W. C., Synthesis, electronic structure, and reactivity of metallacyclotetraazapentadienes. Accounts of Chemical Research 1990, 23 (12), 426-431.

58. Hillhouse, G. L.; Bercaw, J. E., Monosubstituted triazenido complexes as intermediates in the formation of amido complexes from hafnium hydrides and aryl azides. Organometallics 1982, 1 (8), 1025-1029.

59. Zirngast, M.; Flock, M.; Baumgartner, J.; Marschner, C., Group 4 metallocene complexes of disilenes, digermenes, and a silagermene. Journal of the American Chemical Society 2009, 131 (43), 15952-15962.

60. Hlina, J.; Baumgartner, J.; Marschner, C.; Zark, P.; Müller, T., Coordination chemistry of disilylated germylenes with group 4 metallocenes. Organometallics 2013, 32 (11), 3300-3308.

61. Arp, H.; Baumgartner, J.; Marschner, C.; Zark, P.; Müller, T., Coordination chemistry of cyclic disilylated stannylenes and plumbylenes to group 4 metallocenes. Journal of the American Chemical Society 2012, 134 (26), 10864-10875.

62. Lee, V. Y.; Aoki, S.; Yokoyama, T.; Horiguchi, S.; Sekiguchi, A.; Gornitzka, H.; Guo, J.-D.; Nagase, S., Toward a Silicon Version of Metathesis: From Schrock-Type Titanium Silylidenes to Silatitanacyclobutenes. Journal of the American Chemical Society 2013, 135 (8), 2987-2990.

63. Nakata, N.; Fujita, T.; Sekiguchi, A., A stable schrock-type hafnium-silylene complex. Journal of the American Chemical Society 2006, 128 (50), 16024-16025.

64. Stephan, D. W., Early-late heterobimetallics. Coordination chemistry reviews 1989, 95 (1), 41 107.

65. Baranger, A. M.; Bergman, R. G., Cooperative Reactivity in the Interactions of XH Bonds with a Zirconium-Iridium Bridging Imido Complex. Journal Of The American Chemical Society 1994, 116 (9), 3822-3835.

66. Avent, A. G.; Drost, C.; Gehrhus, B.; Hitchcock, P. B.; Lappert, M. F., Synthetic and Structural Studies on the Cyclic Bis (amino) stannylenes $\mathrm{Sn}\left[(\mathrm{NR})_{2} \mathrm{C}_{10} \mathrm{H}_{6}\right.$ ] and their Reactions with $\mathrm{SnCl}_{2}$ or $\mathrm{Si}$ $\left[\left(\mathrm{NCH}_{2}{ }^{t} \mathrm{Bu}\right)_{2} \mathrm{C}_{6} \mathrm{H}_{4}\right]\left(\mathrm{R}=\mathrm{SiMe}_{3}\right.$ or $\left.\mathrm{CH}_{2}{ }^{t} \mathrm{Bu}\right)$. Zeitschrift für anorganische und allgemeine Chemie 2004, 630 (12), 2090-2096.

67. Spinney, H. A.; Yap, G. P.; Korobkov, I.; DiLabio, G.; Richeson, D. S., Construction of a stable Nheterocyclic phosphenium cation with an electron-rich framework and its complexation to rhodium. Organometallics 2006, 25 (15), 3541-3543.

68. Spinney, H. A.; Korobkov, I.; Richeson, D. S., Diamidonaphthalene-supported pnictogenium cations: Synthesis of an N-heterocyclic stibenium cation by a novel protonation route. Chemical Communications 2007, (16), 1647-1649. 
69. Hellmann, K. W.; Galka, C. H.; Rüdenauer, I.; Gade, L. H.; Scowen, I. J.; McPartlin, M., MetalLigand versus Metal-Metal Redox Chemistry: Thallium (I)-Induced Synthesis of 4, 9Diaminoperylenequinone-3, 10-diimine Derivatives. Angewandte Chemie International Edition 1998, 37 (13-14), 1948-1952.

70. Mann, J. B.; Meek, T. L.; Allen, L. C., Configuration energies of the main group elements. Journal of the American Chemical Society 2000, 122 (12), 2780-2783.

71. Fulmer, G. R.; Miller, A. J.; Sherden, N. H.; Gottlieb, H. E.; Nudelman, A.; Stoltz, B. M.; Bercaw, J. E.; Goldberg, K. I., NMR chemical shifts of trace impurities: common laboratory solvents, organics, and gases in deuterated solvents relevant to the organometallic chemist.

Organometallics 2010, 29 (9), 2176-2179.

72. Stender, M.; Phillips, A. D.; Wright, R. J.; Power, P. P., Synthesis and characterization of a digermanium analogue of an alkyne. Angewandte Chemie International Edition 2002, 41 (10), 1785-1787.

73. Jana, A.; Roesky, H. W.; Schulzke, C., Reactivity of germanium (II) hydride with nitrous oxide, trimethylsilyl azide, ketones, and alkynes and the reaction of a methyl analogue with trimethylsilyl diazomethane. Dalton Transactions 2010, 39 (1), 132-138.

74. Alcock, N. W.; Pierce-Butler, M., Crystal and molecular structures of the tetrakis (diphenylketimine) derivatives of silicon, germanium, and tin. Journal of the Chemical Society, Dalton Transactions 1975, (22), 2469-2476.

75. Gottfried, A. C.; Wang, J.; Wilson, E. E.; Beck, L. W.; Banaszak Holl, M. M.; Kampf, J. W., Synthesis of a digermane-containing tricylic nonadecadienedione incorporating an equivalent of ringopened THF. Inorganic chemistry 2004, 43 (24), 7665-7670.

76. Chen, T.; Hunks, W.; Chen, P. S.; Xu, C.; DiPasquale, A. G.; Rheingold, A. L., Synthesis, Structural Characterization, and Thermal Properties of the First Germanium N, N, N', N'-

Tetraalkylguanidinates. Organometallics 2009, 29 (2), 501-504.

77. Shutov, P. L.; Karlov, S. S.; Harms, K.; Tyurin, D. A.; Zabalov, M. V.; Churakov, A. V.; Howard, J. A.; Lorberth, J.; Zaitseva, G. S., X-ray diffraction studies of two azametallatranes. Peculiarities of structures of $\mathrm{N}, \mathrm{N}^{\prime}, \mathrm{N}^{\prime \prime}$-tris (trimethylsilyl) azametallatranes using DFT calculations. Journal of molecular structure 2004, 689 (3), 261-268.

78. Walding, J. L.; Fanwick, P. E.; Weinert, C. S., Synthesis and reactivity of the bulky germanium (IV) trisamide complexes $\mathrm{BrGe}\left[\mathrm{N}\left(\mathrm{SiMe}_{3}\right)_{2}\right]_{3}$ and $\mathrm{LiGe}\left[\mathrm{N}\left(\mathrm{SiMe}_{3}\right)_{2}\right]_{3}$ : X-ray crystal structures of $\mathrm{BrGe}\left[\mathrm{N}\left(\mathrm{SiMe}_{3}\right)_{2}\right]_{3}$ and $\left[\left(\mathrm{Me}_{3} \mathrm{Si}\right)_{2} \mathrm{~N}\right]_{3} \mathrm{Ge}\left(\mathrm{CH}_{2} \mathrm{CH}_{2} \mathrm{CH}_{2} \mathrm{CH}_{3}\right)$. Inorganica Chimica Acta 2005, 358 (4), 1186-1192.

79. Mitzinger, S.; Broeckaert, L.; Massa, W.; Weigend, F.; Dehnen, S., Understanding of multimetallic cluster growth. Nature communications 2016, 7, 10480.

80. Bentlohner, M. M.; Jantke, L. A.; Henneberger, T.; Fischer, C.; Mayer, K.; Klein, W.; Fässler, T. F., On the Nature of Bridging Metal Atoms in Intermetalloid Clusters: Synthesis and Structure of the Metal-Atom-Bridged Zintl Clusters $\left[\mathrm{Sn}\left(\mathrm{Ge}_{9}\right)_{2}\right]_{4}$ and $\left.\left[\mathrm{Zn}\left(\mathrm{Ge}_{9}\right)_{2}\right)\right]_{6}$. Chemistry-A European Journal 2016, 22 (39), 13946-13952.

81. Hull, M. W.; Sevov, S. C., Water compatibility and organic transformations of organo-Zintl deltahedral clusters. Chemical Communications 2012, 48 (62), 7720-7722.

82. Kersting, B.; Krebs, B., Syntheses and Structures of Germanium (II) and Germanium (IV) Thiolate and Selenolate Complexes:[Et $\left.t_{4} \mathrm{~N}\right]\left[\mathrm{Ge}(\mathrm{SPh})_{3}\right],\left[\mathrm{Ph}_{4} \mathrm{P}\right]\left[\mathrm{Ge}(\mathrm{SePh})_{3}\right],\left[\mathrm{Ph}_{4} \mathrm{P}\right]_{2}\left[\mathrm{Ge}_{2}\left(\mathrm{SCH}_{2} \mathrm{CH}_{2} \mathrm{~S}\right)_{3}\right], \mathrm{Ge}(\mathrm{S}-4-$ $\left.\mathrm{MeC}_{6} \mathrm{H}_{4}\right)_{4}$, and $\mathrm{Ge}\left(\mathrm{Se} \mathrm{2,} \mathrm{4,} \mathrm{6-Me}{ }_{3} \mathrm{C}_{6} \mathrm{H}_{2}\right)_{4}$. Examples of the First Anionic Germanium (II) Complexes Containing the Trigonal Pyramidal Ge(II) $\mathrm{S}_{3}$ and $\mathrm{Ge}(\mathrm{II}) \mathrm{Se}_{3}$ Cores. Inorganic Chemistry 1994, 33 (18), 3886-3892.

83. Kociok-Köhn, G.; Winter, J. G.; Filippou, A. C., Trimethylphosphonium trichlorogermanate (II). Acta Crystallographica Section C: Crystal Structure Communications 1999, 55 (3), 351-353. 
84. Godfrey, S. M.; Mushtaq, I.; Pritchard, R. G., Reaction of $\mathrm{GeCl}_{4}$ with some phosphorus and arsenic donor ligands; crystal structures of the germanium (II) complex $\left[{ }^{n} \mathrm{Bu}_{3} \mathrm{PCl}\right]\left[\mathrm{GeCl}_{3}\right]$ and the germanium (IV) complex $\mathrm{GeCl}_{4}\left(\mathrm{AsMe}_{3}\right)_{2}$, the first reported example of a tertiary arsine complex of Ge IV. Journal of the Chemical Society, Dalton Transactions 1999, (8), 1319-1324.

85. Ahlemann, J. T.; Roesky, H. W.; Murugavel, R.; Parisini, E.; Noltemeyer, M.; Schmidt, H. G.; Müller, O.; Herbst-Irmer, R.; Markovskii, L. N.; Shermilovich, Y. G., The Role of the 2, 4, 6-Tris (trifluoromethyl) phenylamino Group in Stabilizing New Phosphorus-, Arsenic-, and GermaniumContaining Main-Group Compounds and Transition-Metal Derivatives. Chemische Berichte 1997, 130 (8), 1113-1121.

86. Merrill, W. A.; Wright, R. J.; Stanciu, C. S.; Olmstead, M. M.; Fettinger, J. C.; Power, P. P., Synthesis and Structural Characterization of a Series of Dimeric Metal (II) Imido Complexes \{M $(\mu-\mathrm{NAr})\}_{2}\left[\mathrm{M}=\mathrm{Ge}, \mathrm{Sn}, \mathrm{Pb} ; \mathrm{Ar}=\mathrm{C}_{6} \mathrm{H}_{3}-2,6-\left(\mathrm{C}_{6} \mathrm{H}_{2}-2,4,6-\mathrm{Me}_{3}\right)_{2}\right]$ and the Related Monomeric Primary Amido Derivatives $\mathrm{M}\{\mathrm{N}(\mathrm{H}) \mathrm{Ar}\}_{2}(\mathrm{M}=\mathrm{Ge}, \mathrm{Sn}, \mathrm{Pb})$ : Spectroscopic Manifestations of Secondary Metal- Ligand Interactions. Inorganic chemistry 2010, 49 (15), 7097-7105.

87. Hitchcock, P. B.; Lappert, M. F.; Thorne, A. J., Novel two-coordinate germanium (II) arylamides: $\mathrm{Ge}(\mathrm{NHAr})_{2}, \mathrm{ArN}[\mathrm{Ge}(\mathrm{NHAr})]_{2}(\mu-\mathrm{NAr})$ and $[\mathrm{Ge}(\mu-\mathrm{NAr})]_{2}$, and the X-ray structures of $\mathrm{Sn}(\mathrm{NHAr})_{2}(\mathrm{Ar}=$ $\left.\mathrm{C}_{6} \mathrm{H}_{2}{ }^{\mathrm{t}} \mathrm{Bu}_{3}-2,4,6\right)$. Journal of the Chemical Society, Chemical Communications 1990, (22), 15871589.

88. Wei, Y.; Wang, S.; Zhou, S.; Feng, Z.; Guo, L.; Zhu, X.; Mu, X.; Yao, F., Aluminum Alkyl Complexes Supported by Bidentate N, N Ligands: Synthesis, Structure, and Catalytic Activity for Guanylation of Amines. Organometallics 2015, 34 (10), 1882-1889.

89. Xue, M.; Zheng, Y.; Hong, Y.; Yao, Y.; Xu, F.; Zhang, Y.; Shen, Q., Catalytic addition of amines to carbodiimides by bis ( $\beta$-diketiminate) lanthanide (II) complexes and mechanistic studies. Dalton Transactions 2015, 44 (46), 20075-20086.

90. Pi, C.; Liu, R.; Zheng, P.; Chen, Z.; Zhou, X., Selective reaction based on the linked diamido ligands of dinuclear lanthanide complexes. Inorg. Chem 2007, 46 (13), 5252-5259.

91. Veith, M.; Becker, S.; Huch, V., An Intramolecularly Lewis-Base-Stabilized Ge N-Compound and a Related Ge-Fe Complex. Angewandte Chemie International Edition 1990, 29 (2), 216-218.

92. Oshita, H.; Mizobe, Y.; Hidai, M., Preparation and properties of molybdenum and tungsten dinitrogen complexes: $X L I$. Silylation and germylation of a coordinated dinitrogen in cis- $\left[M(N)_{2}\right.$ $\left.\left(\mathrm{PMe}_{2} \mathrm{Ph}\right)_{4}\right](\mathrm{M}=\mathrm{Mo}, \mathrm{W})$ using $\mathrm{R}_{3} \mathrm{ECl} / \mathrm{Nal}$ and $\mathrm{R}_{3} \mathrm{ECl} / \mathrm{Na}$ mixtures $(\mathrm{E}=\mathrm{Si}, \mathrm{Ge})$. X-ray structure of trans-[WI $\left.\left(\mathrm{NNGePh}_{3}\right)\left(\mathrm{PMe}_{2} \mathrm{Ph}\right)_{4}\right]\left(\mathrm{C}_{6} \mathrm{H}_{6}\right)$. Journal of organometallic chemistry 1993, 456 (2), 213220.

93. Ellis, D.; Hitchcock, P. B.; Lappert, M. F., Preparation and X-ray structure of $\left[\mathrm{Ge}\left\{\mathrm{N}\left(\mathrm{SiMe}_{3}\right)_{2}\right\}_{2}(\mu-\mathrm{O})\right]$ 2, a rare 1, 3-cyclodigermoxane. Journal of the Chemical Society, Dalton Transactions 1992, (23), 3397-3398.

94. Litz, K. E.; Kampf, J. W.; Banaszak Holl, M. M., Activation of Arylnitroso Substrates on a Platinum- Germylene Complex Facilitating the Formation of New N-C and N-S Bonds. Journal of the American Chemical Society 1998, 120 (30), 7484-7492.

95. Chen, T.; Hunks, W.; Chen, P. S.; Stauf, G. T.; Cameron, T. M.; Xu, C.; DiPasquale, A. G.; Rheingold, A. L., Insertion Reactions of an Aminogermylene and Formation of a Single-Source Precursor for GeTe Thin Films. European Journal of Inorganic Chemistry 2009, 2009 (14), 20472049.

96. Schrick, A. C.; Chen, C.; Rheingold, A. L.; Weinert, C. S., Synthesis of Ge[N(SiMe $\left.\left.{ }_{2} \mathrm{Ph}\right)_{2}\right]_{2}$ and crystal structures of the benzil adducts $\mathrm{Ph}_{2} \mathrm{C}_{2} \mathrm{O}_{2} \mathrm{Ge}\left[\mathrm{N}\left(\mathrm{SiMe}_{2} \mathrm{Ph}\right)_{2}\right]_{2}$ and $\mathrm{Ph}_{2} \mathrm{C}_{2} \mathrm{O}_{2} \mathrm{Ge}\left[\mathrm{N}\left(\mathrm{SiMe}_{3}\right)_{2}\right]_{2}$. Main Group Chemistry 2012, 11 (1), 3-11.

97. Litz, K. E.; Henderson, K.; Gourley, R. W.; Holl, M. M. B., Reversible Insertion Reactions of a Platinum Germylene Complex. Organometallics 1995, 14 (11), 5008-5010. 
98. Cygan, Z. T.; Kampf, J. W.; Banaszak Holl, M. M., Insertion of $\mathrm{SO}_{2}$ into a Sulfide-Bridged $\mathrm{M}-\mathrm{Ge}$ Bond: Synthesis, Characterization, and Reactivity of the O-Germyl-S-sulfoxylate. Inorganic chemistry 2004, 43 (6), 2057-2063.

99. Ossig, G.; Meller, A.; Müller, O.; Herbst-Irmer, R., The Unidentified Product of the Reaction of Dimethyl 2-Diazomalonate with Bis [bis (trimethylsilyl) amino] germylene. Organometallics 1996, 15 (23), 5060-5061.

100. Ohtaki, T.; Kabe, Y.; Ando, W., Reactions of bis [bis (trimethylsilyl) methyl] germylene and the corresponding stannylene with diazidosilanes. Heteroatom Chemistry 1994, 5 (3), 313-320.

101. Rit, A.; Tirfoin, R.; Aldridge, S., Exploiting Electrostatics To Generate Unsaturation: Oxidative GeE Bond Formation Using a Non $\pi$-Donor Stabilized [R(L)Ge: $]^{+}$Cation. Angewandte Chemie 2016, 128 (1), 386-390.

102. Veith, M.; Rammo, A., Synthese von cyclischen, chiralen Verbindungen mit Ge-ElDoppelbindungen $(\mathrm{El}=\mathrm{N}, \mathrm{S},(\mathrm{O}))$ und deren dynamisches Verhalten in Lösung. Zeitschrift für anorganische und allgemeine Chemie 1997, 623 (1-6), 861-872.

103. Cui, C.; Olmstead, M. M.; Fettinger, J. C.; Spikes, G. H.; Power, P. P., Reactions of the Heavier Group 14 Element Alkyne Analogues $\operatorname{Ar}^{\prime} E E A r^{\prime}\left(\mathrm{Ar}^{\prime}=\mathrm{C}_{6} \mathrm{H}_{3}-2,6\left(\mathrm{C}_{6} \mathrm{H}_{3}-2,6-{ }^{i} \mathrm{Pr}_{2}\right)_{2} ; \mathrm{E}=\mathrm{Ge}, \mathrm{Sn}\right)$ with Unsaturated Molecules: Probing the Character of the E-E Multiple Bonds. Journal of the American Chemical Society 2005, 127 (49), 17530-17541.

104. Uhl, W.; Pelties, S.; Rohling, M.; Tannert, J., Alkenyl-Alkynylgermanes Functionalised by Lewis Acids: Intramolecular Aluminium-and Gallium-Alkyne Interactions and Potential Ge-C Bond Activation. European Journal of Inorganic Chemistry 2014, 2014 (17), 2809-2818.

105. Fedushkin, I. L.; Khvoinova, N. M.; Skatova, A. A.; Fukin, G. K., Oxidative Addition of Phenylacetylene through $\mathrm{C}-\mathrm{H}$ Bond Cleavage To Form the $\mathrm{Mg}(\mathrm{II})-\mathrm{dpp}$-bian Complex: Molecular Structure of $\left[\mathrm{Mg}\{\mathrm{dpp}-\operatorname{bian}(\mathrm{H})\}(\mathrm{CCPh})(\mathrm{thf})_{2}\right]$ and Its Diphenylketone Insertion Product $[\mathrm{Mg}(\mathrm{dpp}-$ bian)- $\left\{\mathrm{OC}\left(\mathrm{Ph}_{2}\right) \mathrm{CCPh}\right\}($ thf $\left.)\right]$. Angewandte Chemie International Edition 2003, 42 (42), 5223-5226.

106. Zheng, W.; Pi, C.; Wu, H., An Alkynylaminomethylaluminum Species with a Six-Membered Chair Conformation $\mathrm{Al}_{2} \mathrm{C}_{2} \mathrm{~N}_{2}$ Framework: A New Path to Geminal N/Al Frustrated Lewis Pairs. Organometallics 2012, 31 (10), 4072-4075.

107. Polse, J. L.; Andersen, R. A.; Bergman, R. G., Reactivity of a Terminal Ti (IV) Imido Complex toward Alkenes and Alkynes: Cycloaddition vs $\mathrm{C}-\mathrm{H}$ Activation. Journal of the American Chemical Society 1998, 120 (51), 13405-13414.

108. Blake, R. E.; Antonelli, D. M.; Henling, L. M.; Schaefer, W. P.; Hardcastle, K. I.; Bercaw, J. E., A Cationic Imido Complex of Permethyltantalocene: $\mathrm{H}_{2}$ and Carbon-Hydrogen Bond Activation, [2+2] Cycloaddition Reactions, and an Unusual Reaction with Carbon Dioxide That Affords Coordinated Isocyanate. Organometallics 1998, 17 (4), 718-725.

109. Radovic, L.; Silva, I.; Ume, J.; Menendez, J.; Leon, C. L. Y.; Scaroni, A., An experimental and theoretical study of the adsorption of aromatics possessing electron-withdrawing and electrondonating functional groups by chemically modified activated carbons. Carbon 1997, 35 (9), 13391348.

110. Jilek, R. E.; Tomson, N. C.; Scott, B. L.; Boncella, J. M., [2+2] cycloaddition reactions at terminal imido uranium (IV) complexes to yield isolable cycloadducts. Inorganica Chimica Acta 2014, 422, 78-85.

111. Wildsmith, T.; Hill, M. S.; Johnson, A. L.; Kingsley, A. J.; Molloy, K. C., Exclusive formation of SnO by low temperature single-source AACVD. Chemical Communications 2013, 49 (78), 8773-8775.

112. Paul, F.; Osborn, J. A.; Fischer, J.; Ochsenbein, P., Metallacyclic interconversions in the chemistry of palladium with phenyl isocyanate. Angewandte Chemie International Edition 1993, 32 (11), 1638-1640. 
113. Blum, S. A.; Walsh, P. J.; Bergman, R. G., Epoxide-opening and group-transfer reactions mediated by monomeric zirconium imido complexes. Journal of the American Chemical Society 2003, 125 (47), 14276-14277.

114. Krska, S. W.; Zuckerman, R. L.; Bergman, R. G., Use of steric hindrance and a metallacyclobutene resting state to develop robust and kinetically characterizable zirconium-based imine metathesis catalysts. Journal of the American Chemical Society 1998, 120 (45), 11828-11829.

115. Nugent, W. A.; Haymore, B. L., Transition metal complexes containing organoimido (NR) and related ligands. Coordination Chemistry Reviews 1980, 31 (2), 123-175.

116. King, E. R.; Sazama, G. T.; Betley, T. A., Co (III) imidos exhibiting spin crossover and C-H bond activation. Journal of the American Chemical Society 2012, 134 (43), 17858-17861.

117. Kriegel, B. M.; Bergman, R. G.; Arnold, J., Nitrene Metathesis and Catalytic Nitrene Transfer Promoted by Niobium Bis (imido) Complexes. Journal of the American Chemical Society 2015, $138(1), 52-55$.

118. Kuppuswamy, S.; Powers, T. M.; Johnson, B. M.; Bezpalko, M. W.; Brozek, C. K.; Foxman, B. M.; Berben, L. A.; Thomas, C. M., Metal-Metal Interactions in $\mathrm{C}_{3}$-Symmetric Diiron Imido Complexes Linked by Phosphinoamide Ligands. Inorganic chemistry 2012, 52 (9), 4802-4811.

119. Ossig, G.; Meller, A.; Freitag, S.; Herbst-Irmer, R., Synthesis and X-ray crystal structure of a stannaimine. Journal of the Chemical Society, Chemical Communications 1993, (6), 497-499.

120. Luker, T.; Whitby, R. J.; Webster, M., Insertion of phenyl azide and diphenyldiazomethane into bis (cyclopentadienyl) zirconacyclopentanes. Crystal structure of an 18-electron alkyl zirconocene triazenido complex. Journal of organometallic chemistry 1995, 492 (1), 53-57.

121. Guzei, I. A.; Liable-Sands, L. M.; Rheingold, A. L.; Winter, C. H., Synthesis and characterization of titanium and zirconium complexes bearing diphenyltriazenido ligands. Polyhedron 1997, 16 (23), 4017-4022.

122. Leshinski, S.; Shalumova, T.; Tanski, J. M.; Waterman, R., Insertion reactions involving a triamidoamine-supported zirconium complex. Dalton Transactions 2010, 39 (38), 9073-9078.

123. Cosham, S. D.; Johnson, A. L.; Molloy, K. C.; Kingsley, A. J., Synthesis, characterization, and materials chemistry of group 4 silylimides. Inorganic chemistry 2011, 50 (23), 12053-12063.

124. Nugent, W. A.; Harlow, R., Bridging alkylimido complexes of the Group 4B transition metals. Inorganic Chemistry 1979, 18 (7), 2030-2032.

125. Dolomanov, O. V.; Bourhis, L. J.; Gildea, R. J.; Howard, J. A.; Puschmann, H., OLEX²: a complete structure solution, refinement and analysis program. Journal of Applied Crystallography 2009, 42 (2), 339-341.

126. Li, Y.; Gao, L. X.; Han, F. S., Reliable and Diverse Synthesis of Aryl Azides through CopperCatalyzed Coupling of Boronic Acids or Esters with TMSN 3 . Chemistry-A European Journal 2010, 16 (27), 7969-7972.

127. Lindsay, R., C. F.H. Allen. Organic synthesis 1942, 22, 96.

128. Mtiraoui, H.; Nsira, A.; Msaddek, M.; Renard, P.-Y.; Sabot, C., Regioselective synthesis of otriazolyl-1, 5-benzodiazepin-2-ones and o-isoxazolyl-1, 5-benzodiazepin-2-ones via coppercatalyzed 1, 3-dipolar cycloaddition reactions. Comptes Rendus Chimie 2017.

129. Maity, A. K.; Fortier, S.; Griego, L.; Metta-Magaña, A. J., Synthesis of a "Super Bulky" Guanidinate Possessing an Expandable Coordination Pocket. Inorganic chemistry 2014, 53 (15), 8155-8164.

130. Chorley, R. W.; Hitchcock, P. B.; Lappert, M. F.; Leung, W.-P.; Power, P. P.; Olmstead, M. M., Subvalent Group 14 metal compounds. XIV. The X-ray crystal structures of two monomeric Group 14 metal bisamides, $\mathrm{Ge}\left[\mathrm{N}\left(\mathrm{SiMe}_{3}\right)_{2}\right]_{2}$ and $\mathrm{Sn}\left[\mathrm{NC}(\mathrm{Me})_{2}\left(\mathrm{CH}_{2}\right)_{3} \mathrm{CMe}_{2}\right]_{2}$. Inorganica chimica acta 1992, 198, 203-209.

131. Hunter, W. E.; Hrncir, D. C.; Bynum, R. V.; Penttila, R. A.; Atwood, J. L., The search for dimethylzirconocene. Crystal structures of dimethylzirconocene, dimethylhafnocene, 
chloromethylzirconocene, and (. mu.-oxo) bis (methylzirconocene). Organometallics 1983, 2 (6), 750-755.

132. Liu, F.-C.; Liu, J.; Meyers, E. A.; Shore, S. G., Cyclic Hydroborate Complexes of Metallocenes. IV. Weak Unsupported Single Hydrogen Bridges in $\mathrm{Cp}_{2} \mathrm{Zr}\left\{(\mu-\mathrm{H}) \mathrm{B}(\mathrm{X}) \mathrm{CH}_{2} \mathrm{Ph}\right\}\left\{(\mu-\mathrm{H})_{2} \mathrm{BX}\right\}\left(\mathrm{X}=\mathrm{C}_{5} \mathrm{H}_{10}, \mathrm{C}_{4} \mathrm{H}_{8}\right)$. Inorganic chemistry 1999, 38 (9), 2169-2173.

133. Davies, G. R.; Jarvis, J.; Kilbourn, B.; Pioli, A., Crystal and molecular structure of tetrabenzylzirconium at $-40^{\circ} \mathrm{C}$. Journal of the Chemical Society D: Chemical Communications 1971, (13), 677-677.

134. Zambrano, C. H.; Profilet, R. D.; Hill, J. E.; Fanwick, P. E.; Rothwell, I. P., Aryl imido complexes of the group 4 metals: structural aspects and mechanistic study of formation. Polyhedron 1993, 12 (6), 689-708.

135. Bartlett, R. A.; Power, P. P., Synthesis and structural characterization of the cyclic species [GeN $\left.\left(2,6-i \mathrm{Pr}_{2} \mathrm{C}_{6} \mathrm{H}_{3}\right)\right]_{3}$ : the first" germanazene". Journal of the American Chemical Society 1990, 112 (9), 3660-3662.

136. Hellmann, K. W.; Galka, C.; Gade, L. H.; Kottke, T.; Stalke, D., Aggregation of lithium and mixed thallium (I)-lithium amides through $\eta^{3}$-and $\eta^{6}-\pi$-arene interactions in the solid. Chemical Communications 1998, (5), 549-550. 